\title{
Characterization of the composition and toxicity of particulate matter emissions from advanced heavy-duty natural gas engines
}

Arvind Thiruvengadam Padmavathy

West Virginia University

Follow this and additional works at: https://researchrepository.wvu.edu/etd

\section{Recommended Citation}

Thiruvengadam Padmavathy, Arvind, "Characterization of the composition and toxicity of particulate matter emissions from advanced heavy-duty natural gas engines" (2013). Graduate Theses, Dissertations, and Problem Reports. 542.

https://researchrepository.wvu.edu/etd/542

This Dissertation is protected by copyright and/or related rights. It has been brought to you by the The Research Repository @ WVU with permission from the rights-holder(s). You are free to use this Dissertation in any way that is permitted by the copyright and related rights legislation that applies to your use. For other uses you must obtain permission from the rights-holder(s) directly, unless additional rights are indicated by a Creative Commons license in the record and/ or on the work itself. This Dissertation has been accepted for inclusion in WVU Graduate Theses, Dissertations, and Problem Reports collection by an authorized administrator of The Research Repository @ WVU.

For more information, please contact researchrepository@mail.wvu.edu. 


\title{
CHARACTERIZATION OF THE COMPOSITION AND TOXICITY OF PARTICULATE MATTER EMISSIONS FROM ADVANCED HEAVY-DUTY NATURAL GAS ENGINES
}

\section{ARVIND THIRUVENGADAM PADMAVATHY}

\author{
Dissertation submitted to the \\ College of Engineering and Mineral Resources
}

at West Virginia University

in partial fulfillment of the requirements for the degree of

\author{
Doctor of Philosophy \\ In \\ Mechanical Engineering
}

\author{
Dr. Mridul Gautam \\ Dr. Nigel Clark \\ Dr. Gregory Thompson \\ Dr. Alexsandar Bugarski \\ Dr. Andrew Nix \\ Dr. Mohan Krishnamurthy
}

Department of Mechanical and Aerospace Engineering Morgantown, West Virginia

\section{2}

Keywords: Natural gas, toxicity, stoichiometric, particle size distribution 


\section{ABSTRACT \\ Arvind Thiruvengadam Padmavathy}

Heavy-duty natural gas engines compliant with the 2010 EPA emissions standards have a clear advantage over diesel engines in meeting regulations with minimal after-treatment configuration. Heavy-duty natural gas engines are largely promoted as a cleaner burning engine with respect to low PM mass emissions. However, lack of sufficient data and literature on the exhaust emissions from advanced natural gas engines and the potential adverse health effects has raised concern amongst regulatory agencies. Also, the ammonia emissions from three-way catalyst equipped heavy-duty natural gas engines could be a major contributor to the formation of secondary PM in the atmosphere

This CARB funded study focuses primarily on characterizing the toxicity of the volatile fraction of PM from advanced heavy-duty natural gas engines. The objective of the study also includes characterizing the unregulated species of the exhaust together with number concentration and size distribution of ultrafine nanoparticle emissions. CNG fueled transit buses were tested on WVU's heavy-duty chassis dynamometer in Stockton, CA. A wide array of sampling procedures was included to characterize the complete chemical composition of the exhaust. The toxicity analysis included three different assays a) DHBA b) DTT and c) alveolar macrophage ROS assay.

Results of the gas phase chemical speciation results reported all carbonyl, PAH and VOC emissions close to levels found in background or below the detection limits of the analytical method. Results of elemental analysis reported elements such as calcium, phosphorus, potassium, zinc, sulfur and magnesium are some of the metals that were found in significant concentration in the PM samples. The findings of the study directly relate lubrication oil as the single most dominant source to non-volatile fraction PM emissions in the tailpipe. Both DHBA and DTT assay correlated highly with mass of elements and metals such as zinc, iron and cobalt. The DTT assay resulted in high correlation with mass of copper, zinc, phosphorus and PAH with molecular weight less than 200 . The findings of this study also reported the possible formation of non-volatile nucleation mode particles of $10 \mathrm{~nm}$ size range. 


\section{ACKNOWLEDGMENT}

With a much awaited doctoral education coming to an end, I want to thank a huge group of people who have been with me through this endeavor.

As a student who joined West Virginia University for the master's program, I never imagined PhD was in the list of things to come. The support of my family and their vision has always been my guiding light in achieving this goal. I thank my mom and dad from the bottom of my heart for their continued sacrifices and absolute patience in waiting for me to reach this high point in my education. It should have been difficult for you both to not ask me about my graduation date and patiently wait while I gained my experience in this institution. If not for your unconditional support, my education would have been a mere effort on graduating and not being able to work extensively in this beloved area of research of mine. A special thanks to my brother Pragalath for enduring me as a brother and all the support he has provided.

I extend my sincere appreciation to Dr. Mridul Gautam for being a wonderful advisor in both my studies and in life. Mridul, you have been my family away from home. You have presented me with opportunities and exposure that I am sure would not have received elsewhere. I can say with confidence that your efforts have helped shape my career and future more than mine. I extend my appreciation to Seema for her wonderful hospitality and care.

Next to my family, Sukanya has been my greatest supporter and my biggest well wisher for many years now. She has been a helping hand through many of the troubling phases in my grad student life. I am very fortunate to have such a wonderful friend, who I know will always be there no matter what the problem is. I still believe that it is your advice that prompted me to work under Mridul.

In my long stint as a grad student I have visited many laboratories and worked with different groups. And I am yet to identify a group of people as talented as we have in CAFEE. Every person in this research group is absolutely top notch in skill level, unique in their expertise and posses immense patience while working with graduate students. Dan Carder, you have been a wonderful source of inspiration and great example of a complete engineer. I must thank you for all the assistance that you have selflessly rendered to me and to so many other 
graduate students over the years. One of my largest groups of friends has been people from CAFEE. I must thank Chris Rowe, Jason England, Tom Spencer, Richard Atkinson, Zac Luzader, Brad Ralston, Petr Sindler and Ron Jarrett for all their hard work and continued contribution at the highest level to this research group, while lending a helping hand to every grad student working with CAFEE. Chris and Jason, I must thank you both for introducing me to golf and making every long California trip as a road trip with friends. Marc Besch (the "Swiss guy" or the "Swede") has been one of the most fun filled and talented person I have worked with. It has been an absolute pleasure in exchanging research ideas and working with you on numerous projects. With your meticulous record keeping, it took a lot of pressure of me while working as team.

I must thank my committee members, Dr. Clark, Dr. Thompson, Dr. Bugarski and Dr. Krishnamurthy for their time and effort in vetting this document.

I have been blessed with a great group of friends, who have been long expecting this graduation. Avinas, you have been like a brother to me over the years and I am very thankful for your wonderful support. I would like to thank Hema for being so proud of my achievements. Naveen, Vaithy, Senthil, Srikanth and Praveena it was immense fun hanging around you guys and easily one of the most fun filled years of my life. I hope the best in all your lives.

I must thank my huge family in India for their love and confidence they have had in me. I must thank Reva, TVV uncle and Vandy for the love and affection. 


\section{TABLE OF CONTENTS}

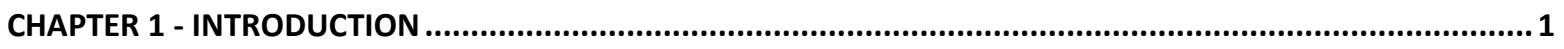

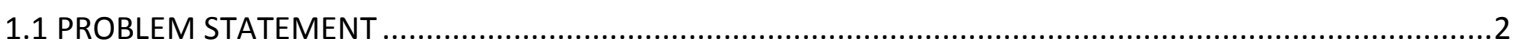

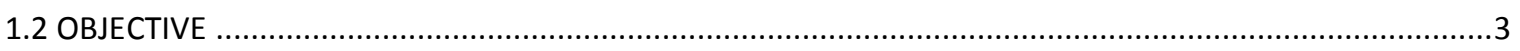

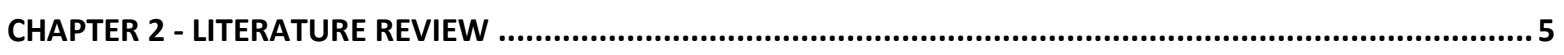

2.1 PM EMISSIONS FROM HEAVY-DUTY DIESEL AND NATURAL GAS ENGINES...........................................

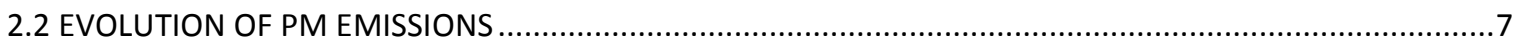

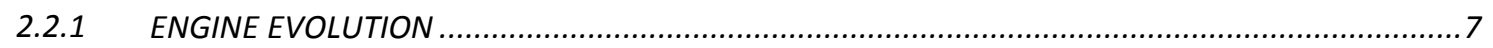

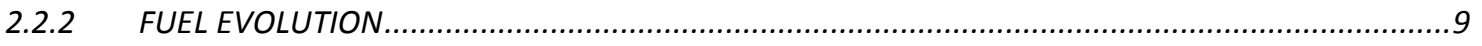

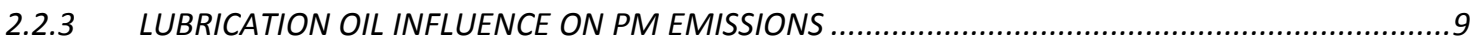

2.2.4 AFTER-TREATMENT SYSTEM INFLUENCE ON PM EMISSIONS …..........................................11

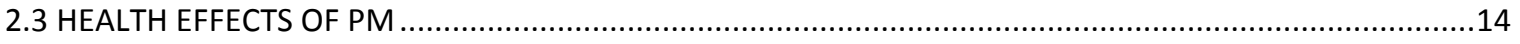

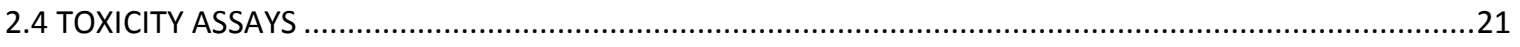

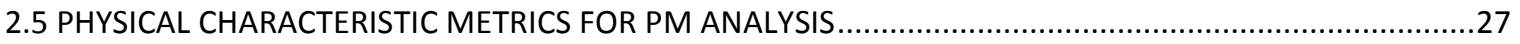

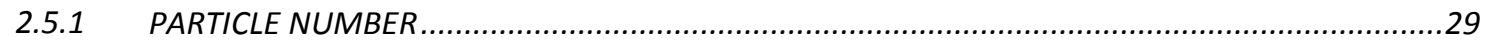

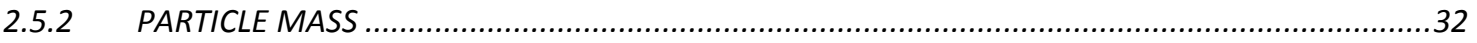

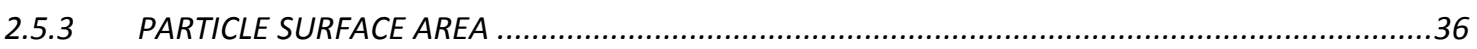

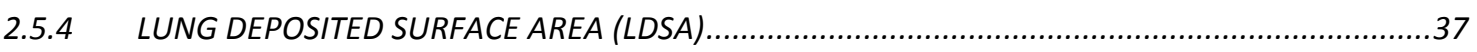

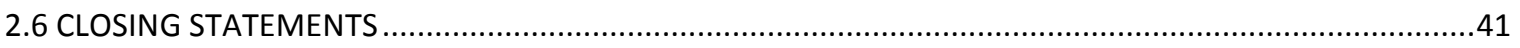

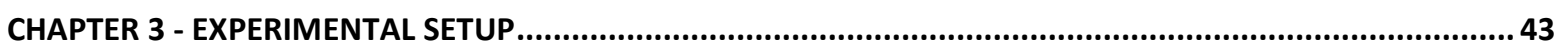

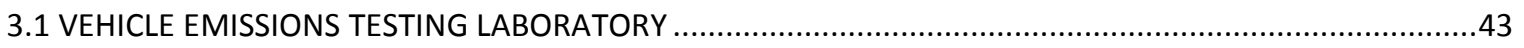

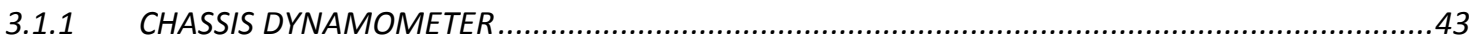

3.1.2 TRANSPORTABLE EMISSIONS MEASUREMENT SYSTEM (TEMS)..........................................47

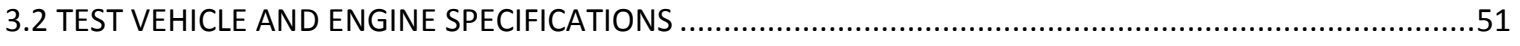

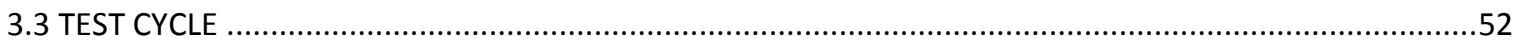

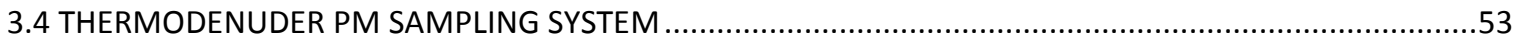

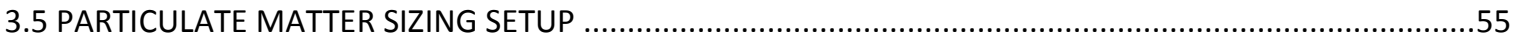

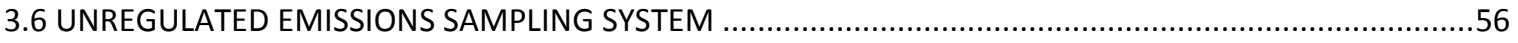

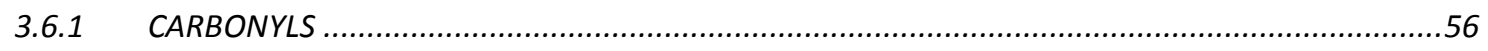

3.6.2 POLYCYCLIC AROMATIC HYDROCARBON (PAH) SAMPLING SYSTEM.........................................57

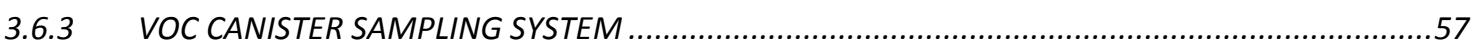

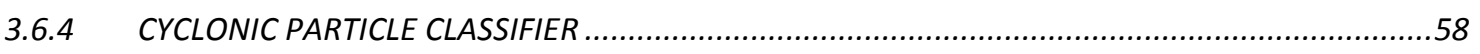

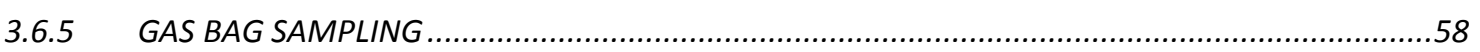




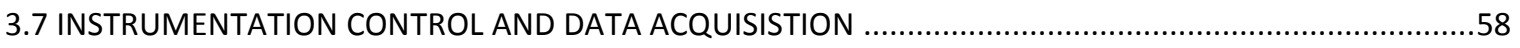

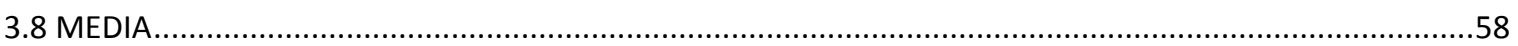

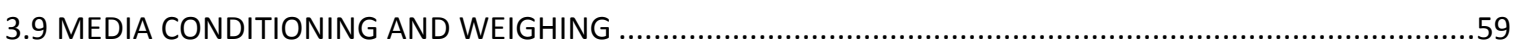

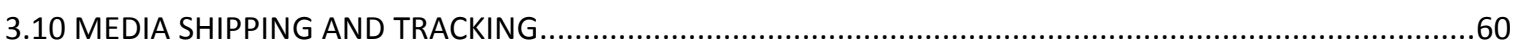

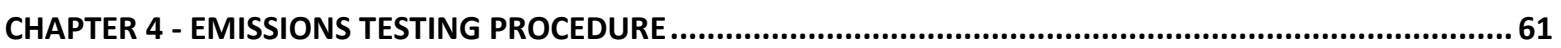

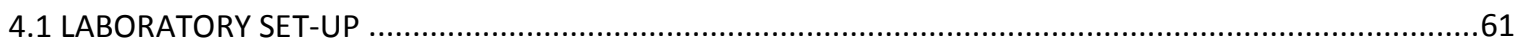

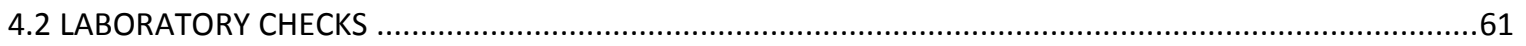

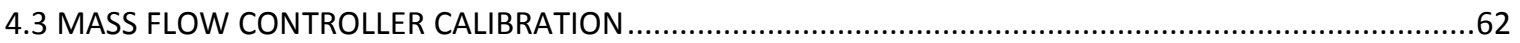

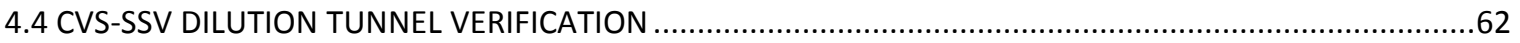

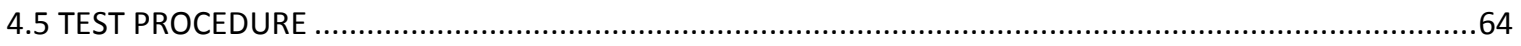

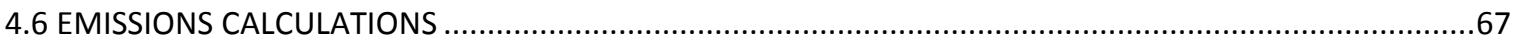

4.6.1 EQUATIONS TO CALCULATE DISTANCE SPECIFIC MASS OF UNREGULATED SPECIES.................67

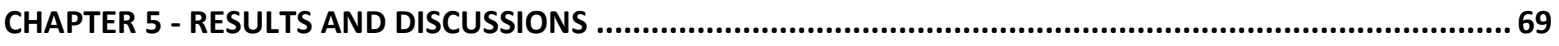

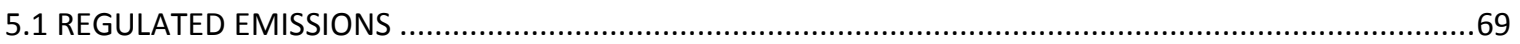

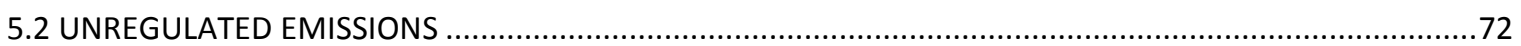

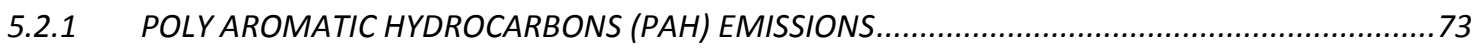

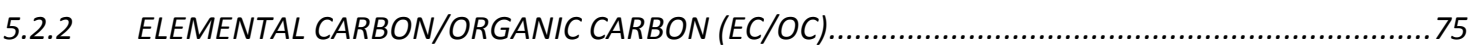

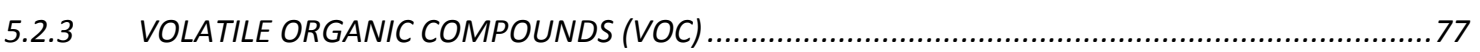

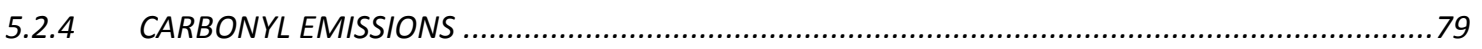

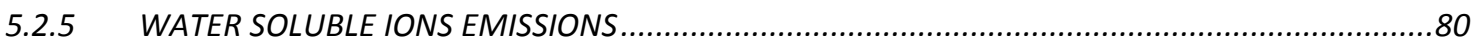

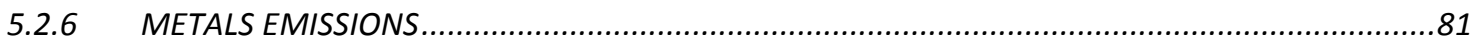

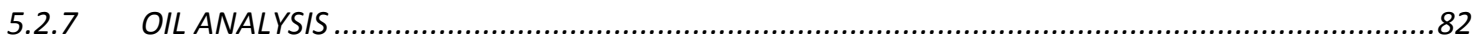

5.3 TOXICITY RESULTS

5.3.1 DTT ASSAY

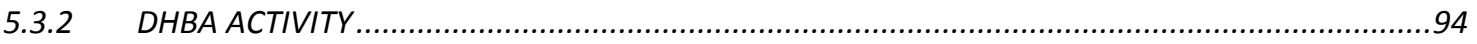

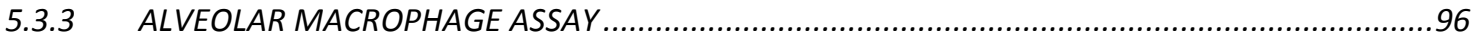

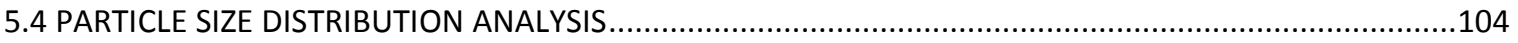

5.4.1 RESULTS OF PARTICLE SIZE DISTRIBUTION ANALYSIS FROM BUS 1......................................105

5.4.2 RESULTS OF PARTICLE SIZE DISTRIBUTION ANALYSIS FROM BUS 2...................................115

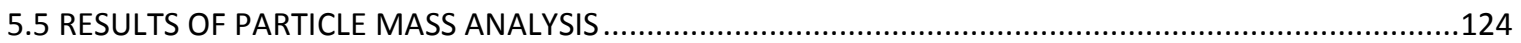

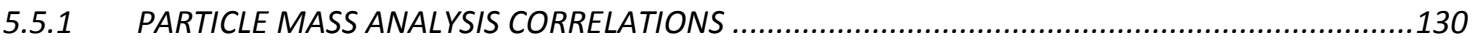

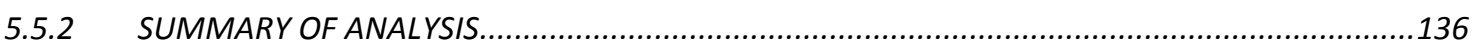

5.6 REGIONAL DEPOSITION FRACTIONS AND PARTICLE SURFACE AREA ANALYSIS ....................................138 
5.6.1 RELATIONSHIP BETWEEN ELECTRICAL AEROSOL DETECTOR RESPONSE TO PARTICLE SURFACE AREA $\quad 150$

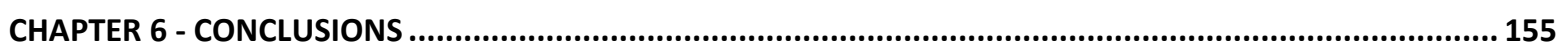

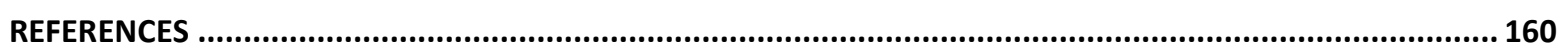

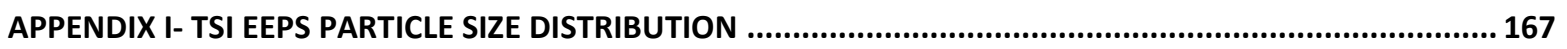

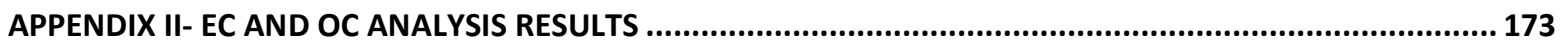

APPENDIX III- ICP-MS ANALYSIS RESULTS OF METALS AND ELEMENTS .................................................... 178

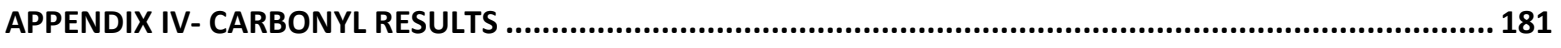

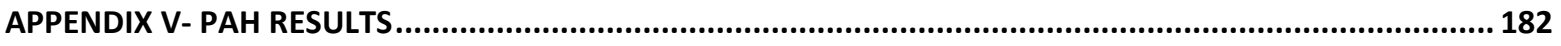

APPENDIX V- DTT ANALYSIS RESULTS

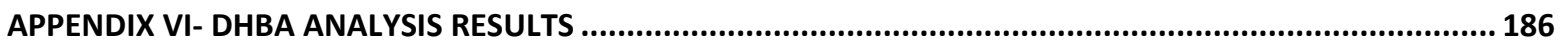

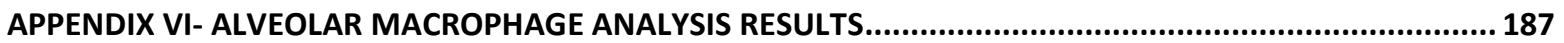




\section{LIST OF TABLES}

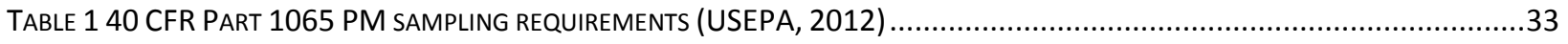

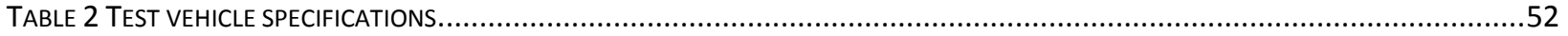

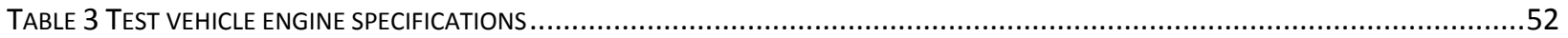

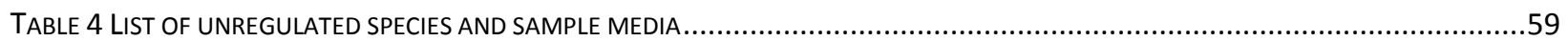

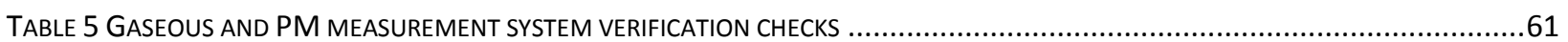

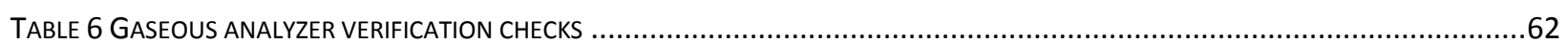

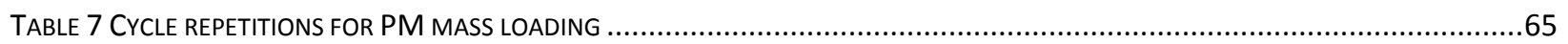

TABLE 8 SUMMARY OF SAMPLING MEDIA, SAMPLING DEVICES AND METHODS USED TO ANALYZE UNREGULATED EXHAUST SPECIES ...........66

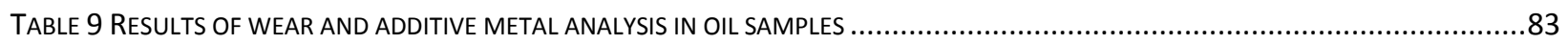

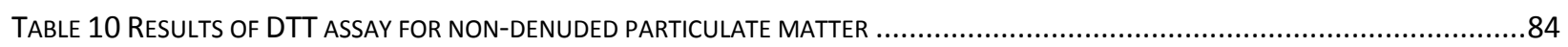

TABle 11 PeARson CoRRELATION COEfFiCIENT (R) AND LEVEL Of SIGNIFICANCE (P) FOR ELEMENTAL SPECIES Vs. DTT ACTIVITY ...........89

TABle 12 PEARSON CORRELATION FOR VOLATILE, SEMI-VOLATILE AND ELEMENTAL CARBON COMPOUNDS VS. DTT ACTIVITY.................91

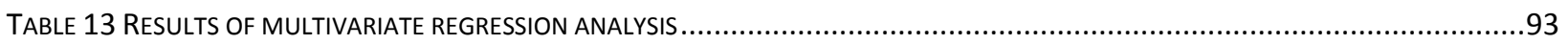

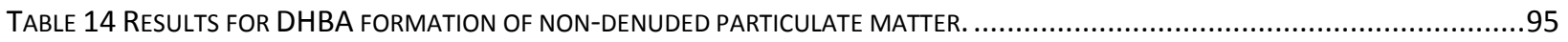

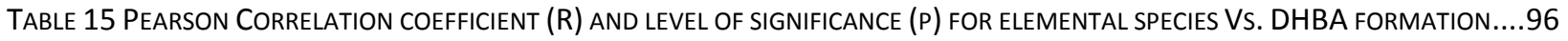

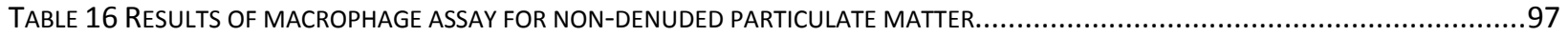

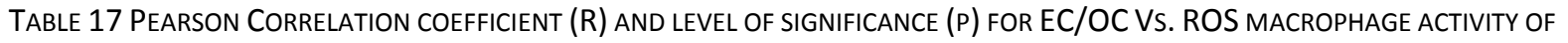
NON-DENUDED PM

TABLE 18 PeARson CoRrelation COEfFiCIENT (R) AND LEVEL OF SIGNIFICANCE (P) FOR ELEMENTAL SPECIES VS. MACROPHAGE ROS

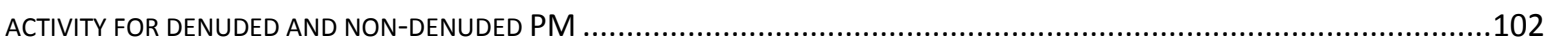

TABLE 19 MULTIVARIATE REGRESSIONS ANALYSIS RESULTS FOR ELEMENTAL COMPOSITION AND MACROPHAGE ROS ACTIVITY..............103

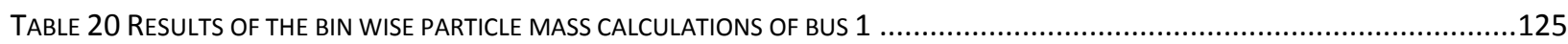

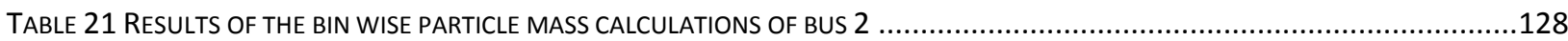

TAble 22 Results of PeARson CORRELATION COEFFICIENT AND LEVEL OF SIGNIFICANCE BETWEeN ELEMENTAL EMISSIONS AND BIN WISE PM MASS EMISSIONS. .130

TAble 23 Results of PeARSON CORRELATION COEFFICIENT AND LEVEL OF SIGNIFICANCE NP MASS AND DTT AND ROS RESPONSES ....134

TABLE 24 TABULATED SUMMARY OF CORRELATION AND STATISTICAL SIGNIFICANCE OF DIFFERENT COMPONENTS ANALYZED ................137

TABLE 25 REgIONAL DEPOSITION FRACTIONS FOR BUS 1 FOR THE THREE DIFFERENT SIZE BINS OVER THE UDDS CYCLE .....................140

TABLE 26 REGIONAL DEPOSITION FRACTIONS FOR BUS 1 FOR THE THREE DIFFERENT SIZE BINS OVER THE STEADY STATE 45 MPH CYCLE.140

TABLE 27 REGIONAL DEPOSITION FRACTIONS FOR BUS 1 FOR THE THREE DIFFERENT SIZE BINS OVER THE IDLE OPERATION...................141

TABLE 28 REGIONAL DEPOSITION FRACTIONS FOR BUS 2 FOR THE THREE DIFFERENT SIZE BINS OVER UDDS CYCLE ..........................141

TABLE 29 REGIONAL DEPOSITION FRACTIONS FOR BUS 2 FOR THE THREE DIFFERENT SIZE BINS OVER THE STEADY STATE 45 MPH CYCLE ..142

TABLE 30 REGIONAL DEPOSITION FRACTIONS FOR BUS 2 FOR THE THREE DIFFERENT SIZE BINS OVER THE IDLE OPERATION. 142 
TABLE 31 PEARSON'S CORRELATION COEFFICIENT (R) AND LEVEL OF SIGNIFICANCE (P) FOR CORRELATION OF EAD DATA VS. REGIONAL AND LUNG DEPOSITED SURFACE AREA AND PARTICLE NUMBER COUNT IN DIFFERENT SIZE BINS ............................................151 


\section{LIST OF FIGURES}

Figure 2-1 TyPiCAL COMPOSITION OF PM FROM A LATE 1990 DieSEL ENGINE TESTEd ON A FTP CYCLE (KITTELSON, 1998B).

FIGURE 2-2 EFFECT OF VGT ON ENGINE OUT PARTICLE SIZE DISTRIBUTION FROM DIESEL ENGINE AND CONCENTRATION (ARDANESE, 2008)

FIGURE 2-3 EFFECT OF EGR VALVE POSITION ON ENGINE OUT PARTICLE SIZE DISTRIBUTION FROM DIESEL ENGINE AND CONCENTRATION (ARDANESE, 2008)

Figure 2-4 CORRELATION OF COPPER CONCENTRATION IN AMBIENT AND DIESEL PM TO DHBA FORMATION RATE (DISTEFANO ET AL., 2009).

FiguRE 2-5 TYPICAL PARTICLE MASS AND NUMBER WEIGHTED DISTRIBUTION (KITTELSON, 1998B) .........................................28

FIGURE 2-6 EFFECTIVE DENSITY FUNCTIONS DEVELOPED BY VARIOUS RESEARCHERS (LIU ET AL., 2012) .......................................35

FIGURE 2-7 MEASURED AND THEORETICAL CURVE FOR PARTICLE DF AS ADAPTED FROM (ENSOR, 2011) ......................................39

Figure 2-8 PerCENTAGe DF CURVE AS Function Of PARTICLE DiAMETER AdAPTED FROM (WILSON ET AL., 2007) ........................41

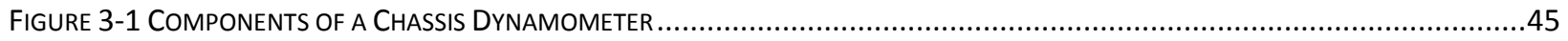

FIGURE 3-2 CONNECTING AND SUPPORTING STRUCTURE OF CHASSIS DYNAMOMETER ..................................................45

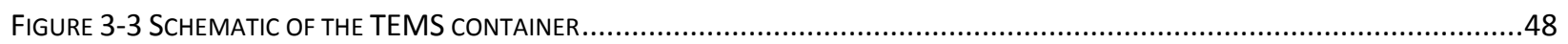

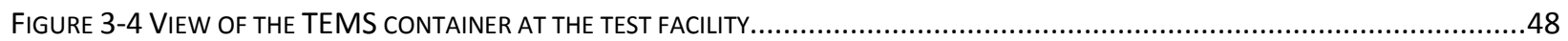

FIgURE 3-5 SCHEMATIC OF THE CFR 40 PART 1065 REgULATEd PM SAMPLING SYSTEM ....................................................50

FIGURE 3-6 CFR 40 PART 1065 COMPLIANT PM SAMPLING SYSTEM ON-BOARD THE TRANSPORTABLE LABORATORY CONTAINER ...........50

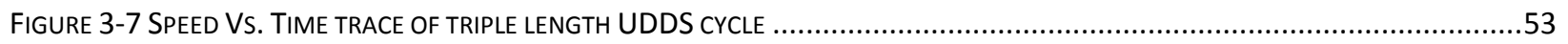

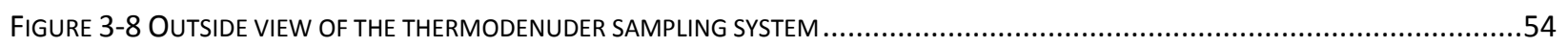

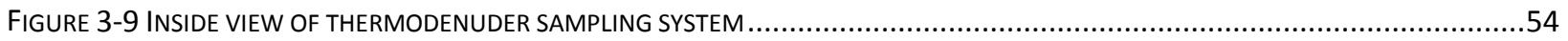

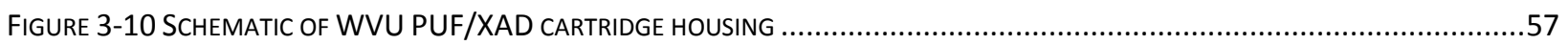

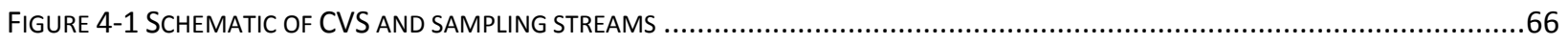

FIGURE 5-1 AXLE WORK-SPECIFIC REGULATED EMISSIONS SUMMARY OF TEST VEHICLES OVER THREE DIFFERENT DRIVING CYCLES............71

FIGURE 5-2 DISTANCE SPECIFIC REGULATED EMISSIONS SUMMARY OF TEST VEHICLES OVER THREE DIFFERENT DRIVING CYCLES. .............72

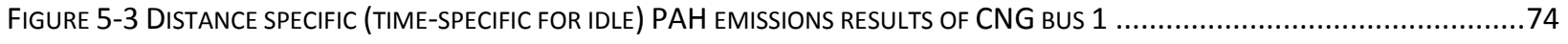

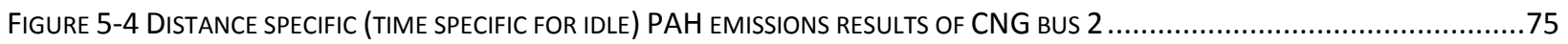

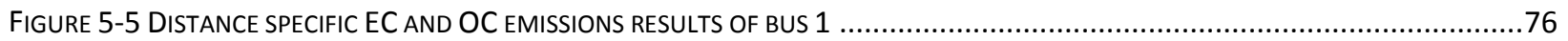

FIGURE 5-6 DISTANCE SPECIFIC BTEX EMISSIONS RESULTS FROM BUS 1 AND BUS 2 .....................................................

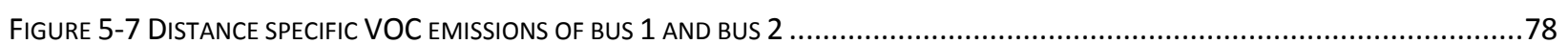

FIGURE 5-8 DISTANCE SPECIFIC (TIME-SPECIFIC FOR IDLE) CARBONYL EMISSIONS RESULTS FOR CNG BUS1 AND BUS $2 \ldots \ldots \ldots \ldots \ldots \ldots . . . . . . . .79$

FIGURE 5-9 DISTANCE SPECIFIC (TIME-SPECIFIC FOR IDLE) WATER SOLUBLE ION EMISSIONS RESULTS OF CNG BUS 1 AND BUS $2 \ldots . . . . . . . . . .81$

FIGURE 5-10 DISTANCE SPECIFIC (TIME-SPECIFIC FOR IDLE) METALS EMISSIONS RESULTS OF CNG BUS 1 AND BUS 2.......................82

FIGURE 5-11 RESULTS OF DTT CONSUMPTION RATE FOR DIFFERENT DRIVING CYCLES....................................................8

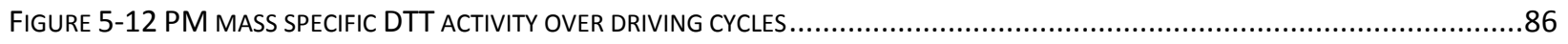


Figure 5-13 Ambient DTt activity levels in Boyle Heights, CA (Cho et Al., 2005)

Figure 5-14 AMBIENT DTT ACTIVITY LEVELS In CALREMONT, CA (CHO ET AL., 2005) .........................................................87

FIGURE 5-15 SCATTER PLOT OF TOTAL PM EMISSION RATES OVER DIFFERENT DRIVING CYCLE VS. DTT CONSUMPTION RATE..................88

FIGURE 5-16 SCATTER PLOT OF THE PREDICTED DTT ACTIVITY VS. MEASURED DTT ACTIVITY ....................................................93

FIGURE 5-17 PM MASS SPECIFIC MACROPHAGE ROS ACTIVITY OVER DIFFERENT DRIVING CYCLES .............................................98

FIGURE 5-18 PM MASS SPECIFIC MACROPHAGE ROS ACTIVITY OF DIFFERENT TECHNOLOGY VEHICLES AND AMBIENT PM (VERMA ET AL., 2010) . .98

FIGURE 5-19 SCATTER PLOT OF MACROPHAGE ROS ACTIVITY VS. ELEMENTAL CARBON MASS .................................................99

FIGURE 5-20 SCATTER PLOT OF THE PREDICTED MACROPHAGE ROS ACTIVITY VS. MEASURED MACROPHAGE ROS ACTIVITY ................104

FIGURE 5-21 RESULTS OF INTEGRATED PARTICLE SIZE DISTRIBUTION AND CONCENTRATION OVER UDDS CYCLE FROM BUS $1 \ldots . . . . . . . . . .105$

Figure 5-22 Bus 1 A) TRANSIENT NP BIN CMD AND CORRESPONDING AEROSOl LENGTH B) TRANSIENT UFP BIN CMD AND CORRESPONDING AEROSOL LENGTH C) TRANSIENT FP BIN CMD AND CORRESPONDING AEROSOL LENGTH D) UDDS CYCLE SPEED

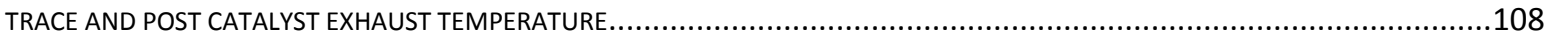

FIGURE 5-23 RESULTS OF INTEGRATED PARTICLE SIZE DISTRIBUTION AND CONCENTRATION DURING IDLE OPERATION OF BUS $1 \ldots . . . . . . . .110$ FIGURE 5-24 RESULTS OF INTEGRATED PARTICLE SIZE DISTRIBUTION AND CONCENTRATION OVER THE STEADY STATE 45 MPH CYCLE FROM BUS 1 .

FIGURE 5-25 BUS 1 STEADY STATE 45 MPH A) NP BIN CMD AND CORRESPONDING AEROSOL LENGTH B) UFP BIN CMD AND CORRESPONDING AEROSOL LENGTH C) FP BIN CMD AND CORRESPONDING AEROSOL LENGTH D) ENGINE PERCENT LOAD AND POST CATALYST EXHAUST TEMPERATURE. 114

FIGURE 5-26 RESULTS OF INTEGRATED PARTICLE SIZE DISTRIBUTION AND CONCENTRATION OVER THE UDDS CYCLE OF BUS 2 115

FIgURE 5-27 Bus 2 A) TRANSIENT NP BIN CMD AND CORRESPONDING AEROSOl LENGTH B) TRANSIENT UFP BIN CMD AND CORRESPONDING AEROSOL LENGTH C) TRANSIENT FP BIN CMD AND CORRESPONDING AEROSOL LENGTH D) UDDS CYCLE SPEED TRACE AND POST CATALYST EXHAUST TEMPERATURE.

FIGURE 5-28 RESULTS OF INTEGRATED PARTICLE SIZE DISTRIBUTION AND CONCENTRATION OVER IDLE OPERATION OF BUS $2 .$. .118

FIGURE 5-29 GRAVIMETRIC FILTER SAMPLE AFTER 9 HOURS OF PM SAMPLING DURING THE IDLE OPERATION ......

FIGURE 5-30 BUS 2 IDLE OPERATION A) NP BIN CMD AND CORRESPONDING AEROSOL LENGTH B) UFP BIN CMD AND CORRESPONDING AEROSOL LENGTH C) FP BIN CMD AND CORRESPONDING AEROSOL LENGTH ...................................................119

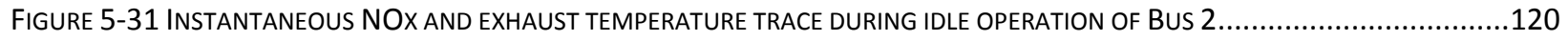

FIGURE 5-32 RESULTS OF INTEGRATED PARTICLE SIZE DISTRIBUTION AND CONCENTRATION OVER STEADY STATE 45 MPH OPERATION OF BUS 2 122

FigURE 5-33 BUS 2 STEAdY STATE 45 MPH OPERATION A) NP BIN CMD AND CORRESPONDING AEROSOL LENGTH B) UFP BIN CMD AND CORRESPONDING AEROSOL LENGTH C) FP BIN CMD AND CORRESPONDING AEROSOL LENGTH D) ECU ENGINE PERCENT LOAD AND

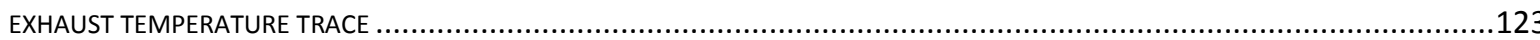

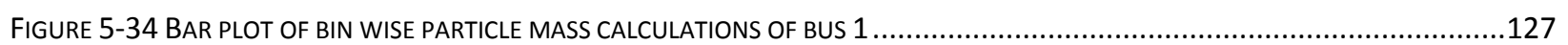

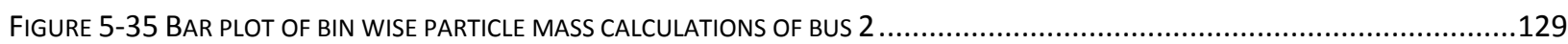


FIGURE 5-36 CHART ADAPTED FROM (LEE ET AL., 2006) SHOWING THE IRON BASED NUCLEATION MODE FOR A DIESEL FUEL DOPED WITH

60 PPM IRON

FIGURE 5-37 REGIONAL AND TOTAL DEPOSITION FRACTIONS CALCULATED FOR THE ELECTRICAL MOBILITY DIAMETER RANGE FOR THE TSI EEPS INSTRUMENT.

FIGURE 5-38 TOTAL PARTICLE SURFACE AREA CALCULATED FOR THE MEASURED CMD IN EACH SIZE BIN 144

FIGURE 5-39 TRACHEOBRONCHIAL AND ALVEOLAR DEPOSITED SURFACE AREA CALCULATED FOR THE MEASURED CMD IN EACH SIZE BIN 144 FIGURE 5-40 PARTICLE SURFACE AREA DISTRIBUTION, REPRESENTING ENGINE-OUT, LUNG DEPOSITED, TRACHEOBRONCHIAL AND ALVEOLAR DEPOSITION FROM BUS 1 OVER THE UDDS CYCLE.

FIGURE 5-41 PARTICLE SURFACE AREA DISTRIBUTION, REPRESENTING ENGINE-OUT, LUNG DEPOSITED, TRACHEOBRONCHIAL AND ALVEOLAR DEPOSITION FROM BUS 1 DURING IDLE OPERATION. 146

FIGURE 5-42 PARTICLE SURFACE AREA DISTRIBUTION, REPRESENTING ENGINE-OUT, LUNG DEPOSITED, TRACHEOBRONCHIAL AND ALVEOLAR DEPOSITION FROM BUS 1 DURING STEADY STATE 45 MPH OPERATION.

FIGURE 5-43 PARTICLE SURFACE AREA DISTRIBUTION, REPRESENTING ENGINE-OUT, LUNG DEPOSITED, TRACHEOBRONCHIAL AND ALVEOLAR DEPOSITION FROM BUS 2 OVER THE UDDS CYCLE.

FIGURE 5-44 PARTICLE SURFACE AREA DISTRIBUTION, REPRESENTING ENGINE-OUT, LUNG DEPOSITED, TRACHEOBRONCHIAL AND ALVEOLAR DEPOSITION FROM BUS 2 DURING STEADY STATE 45 MPH OPERATION.

FIGURE 5-45 PARTICLE SURFACE AREA DISTRIBUTION, REPRESENTING ENGINE-OUT, LUNG DEPOSITED, TRACHEOBRONCHIAL AND ALVEOLAR DEPOSITION FROM BUS 2 DURING IDLE OPERATION. 149

FIGURE 5-46 SCATTER PLOT AND LINEAR FIT OF EAD DATA VS. A) NP SIZE BIN TOTAL LUNG DEPOSITED PARTICLE SURFACE AREA B) NP SIZE BIN ALVEOLAR DEPOSITED PARTICLE SURFACE AREA C) NP SIZE BIN TRACHEOBRONCHIAL DEPOSITED PARTICLE SURFACE AREA ......152

FIGURE 5-47 SCATTER PLOT AND LINEAR FIT OF EAD DATA VS. A) UFP SIZE BIN TOTAL LUNG DEPOSITED PARTICLE SURFACE AREA B) UFP SIZE BIN ALVEOLAR DEPOSITED PARTICLE SURFACE AREA C) UFP SIZE BIN TRACHEOBRONCHIAL DEPOSITED PARTICLE SURFACE AREA

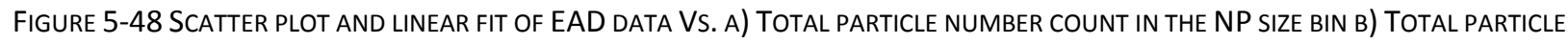
NUMBER COUNT IN THE UFP SIZE BIN. 154 


\section{CHAPTER 1 - INTRODUCTION}

The health effects of ultrafine particulate matter (PM) emissions from internal combustion engines have been a subject of intense debate and scrutiny. The complex chemical and physical nature of PM has contributed to uncertainties about the contribution of the different fractions of PM to toxicity responses. Given the link published between PM surface area and health effects (Wichmann and Peters, 2000, Nemmar et al., 2002), future PM regulation may eventually move towards a particulate number based system in addition to the existing mass based system. With continuous advancements in after-treatment technology and advanced combustion strategies, mass emissions of PM have decreased by orders of magnitude compared to legacy diesel engines. However, increased nanoparticle emissions have been observed over certain operating conditions (Ardanese et al., 2009). The observed nanoparticle emissions could be as result of gas to particle conversions or solid carbon emissions due to momentary loss in diesel particulate filter (DPF) filtration efficiencies. As a first step the European Union has proposed a particle number regulation which is aimed at regulating the number concentration of only the solid fraction of PM. A principle driving force for this regulation has been that elemental carbon emissions have been identified to be active participant in forcing climate change and induce long term health effects (Andersson et al., 2010). However, a significant portion of the heavy-duty vehicle exhausts in the form of volatile and semi-volatile fraction, which can be also attributed to adverse health effects, is not accounted for. The reason for exclusion of this fraction from the proposed regulation is the fact that formation and measurement of volatile nanoparticles are associated with many uncertainties. Looking beyond the issues concerning uncertainties in formation and measurement, lack of sufficient information related to potential health effects of volatile particles has raised concerns among regulatory agencies.

USEPA 2010 emissions compliant heavy-duty engines have adopted advanced combustion strategies and after-treatment technologies to meet current regulations. The diesel segment of the heavy-duty population has adopted exhaust gas recirculation (EGR), variable geometry turbochargers (VGT), diesel particulate filter (DPF) and selective catalytic reduction (SCR) systems while the natural gas segment has adopted a stoichiometric fuelling, coupled with

$$
\text { 1 I P a g e }
$$


cooled EGR strategy, and equipped with a three-way catalyst (TWC) for NOx control. Natural gas engines do not require any PM after-treatment device as the gaseous fuel burns relatively soot free when compared to diesel combustion. PM emissions from both DPF equipped diesel and natural gas vehicles are largely similar in elemental carbon emissions. However, nanoparticle emissions as a result of nucleation of volatile organic compounds and formation of sulfuric acid particles are still significant at certain operating conditions of the engine. Also, the contribution of lubrication oil towards particle formation is of significance as it can prove to be a source of sulfur, poly aromatic hydrocarbon and inorganic precursors to PM formation. Lubrication oil emissions can also be viewed as a major contributor to mass and particle number emissions from natural gas engines as they are not equipped with a particulate filter. Hence, engine durability issues and operating load characteristics could alter the PM emissions rates and characteristics of heavy-duty natural gas engines.

Studies have shown the dependence of exhaust temperatures on nanoparticle emissions from DPF and SCR equipped diesel engines (Kittelson et al., 2008, Vaaraslahti et al., 2005, Thiruvengadam et al., 2012). Findings in literature have shown that even with the use of ultra low sulfur diesel (ULSD) in on-road heavy-duty diesel engines, significant concentrations of nucleation mode particles, believed to be sulfuric acid based are observed (Vaaraslahti et al., 2005, Kittelson et al., 2008). Literature has attributed the sulfur contribution to be primarily from lubrication oil. Sulfur from lubrication oil undergoes sequential oxidation at high exhaust temperatures over catalyzed after-treatment systems to $\mathrm{SO}_{3}$ which upon mixing with moisture in dilution air could form sulfuric acid based nucleation mode particles. However, in the absence of high exhaust temperatures, particle concentrations have been observed to be close to the detection limits of the instrument.

\subsection{PROBLEM STATEMENT}

One of the key questions remaining is the compositional significance of nanoparticle emissions from heavy-duty engines in relation to their toxicity. A compositionally diverse heavyduty PM imparts significant uncertainty to the relative contribution of different fractions to overall PM toxicity. The lack of clarity in health effects of PM from advanced heavy-duty

\section{I P a g e}


engines, is due to the uncertainties in particle formation mechanisms as a result of the presence of multiple after-treatment systems in the form of DPF, SCR and TWC. This study would like focuses on natural gas engines, which have tailpipe PM emissions profiles similar to those of DPF equipped diesel. The study realizes that there are technological differences between natural gas engine and diesel engines. However the investigation of PM from natural gas fueled engines devoid of fuel sulfur would better illustrate the contribution of lubrication oil to engine out PM.

This study used a thermal denuder method of treating a sample stream and removing the volatile fraction through adsorption on an activated charcoal bed. Although the method has its limitations, it is the only method of volatile fraction removal without a catalytic system that might contribute to PM artifacts. Due to the low PM mass emissions from the vehicles, high flow sampling systems were developed to reduce collection times, and have sufficient sample loading for toxicity analysis.

\subsection{OBJECTIVE}

The global objective of this study is to investigate the linkages between the different fractions of PM to their respective toxicity responses.

The study funded by California Air Resources Board involved the collection of PM in a denuded (volatile fraction removed) and non-denuded (total particulate matter) composition to correlate the fractional composition of PM to its toxicity responses. The objectives of the study included the characterization of volatile organic compounds (VOC), polyaromatic hydrocarbons (PAH), carbonyl compounds, elemental carbon, organic carbon, metals and ions emissions.

The study involved the analysis of PM for three different bio assays namely, two cell free) (chemical based) assays 1) didydroxybenzoate (DHBA) assay 2) dithiothreitol (DTT) assay and a cell based alveolar macrophage ROS assay (Reactive Oxidative Species).

Two compressed natural gas (CNG) fueled heavy-duty buses equipped with a three-way catalyst were tested on the WVU heavy-duty chassis dynamometer. The natural gas vehicles were powered by a Cummins ISLG 280 stoichiometrically fueled, cooled EGR engine equipped 
with a three-way catalyst. The Transportable Emissions Measurement System (TEMS) was stationed in Stockton, CA, to carry out the emissions measurement from the two buses 


\section{CHAPTER 2 - LITERATURE REVIEW}

Particulate matter (PM) composition from advanced heavy-duty engines has undergone complex chemical and physiological changes in comparison to PM emitted from legacy diesel engines. A variety of after-treatment systems and combustion strategies employed to meet regulations has largely contributed to this change in composition and emission rate of PM. Primary PM formation from heavy-duty vehicle exhaust depends on the type of fuel, engine operating loads and after-treatment system performance. The literature review in this work will look into the differences in physical and chemical characteristics of PM between diesel and natural gas vehicles and focus on the effect of individual factors namely lubrication oil consumption and after-treatment systems that contribute to PM emissions.

\subsection{PM EMISSIONS FROM HEAVY-DUTY DIESEL AND NATURAL GAS ENGINES}

PM emitted from diesel engines is a complex mixture of volatile, semi-volatile, carbon fractions and inorganic elements. In a diesel engine, the primary PM formed is a result of soot formation within the rich zones of the injector spray cone. Immediately downstream of the combustion chamber, PM is comprised of only soot and metallic fractions. However, precursors for formation of semi-volatile and volatile PM remain in gas phase, until the exhaust undergoes cooling upon dilution with ambient air. Also, of importance is the adsorption of volatile materials on to solid fractions, contributing to particles that may contain a solid core coated with a hydrocarbon layer. The bulk of the organic and volatile fractions of PM are formed as a result of exhaust gas cooling. PAH compounds are one of the dominant precursors for soot formation. They are usually a direct result of incomplete combustion of a fuel high in aromatic content such as diesel. As a result the combustion of diesel, inherently results in higher soot content in comparison to natural gas fuel, which is devoid of any aromatic content (Lev-On et al., 2002). The primary PM formed in the combustion chamber of an internal combustion engine also depends on various other factors such as engine control parameters and load. Kittelson's work has shown the typical composition of a heavy-duty PM from a late 1990 model year diesel engine (Figure 2-1). The results show a composition consisting predominantly of elemental carbon and unburned oil and fuel. The results also show a 
significant contribution of sulfate and water, even with the use of ultra low sulfur diesel (ULSD) with a sulfur percentage typically below $0.05 \%$ by wt (Kittelson, 1998b). The results shown in this study, present the composition of PM collected in accordance with the operational definition of PM by the EPA. Presence of a sulfate component in PM is heavily dependent on moisture content in the exhaust and dilution air, and does not necessarily manifests itself in the total PM every time during real-world operating and dilution conditions.

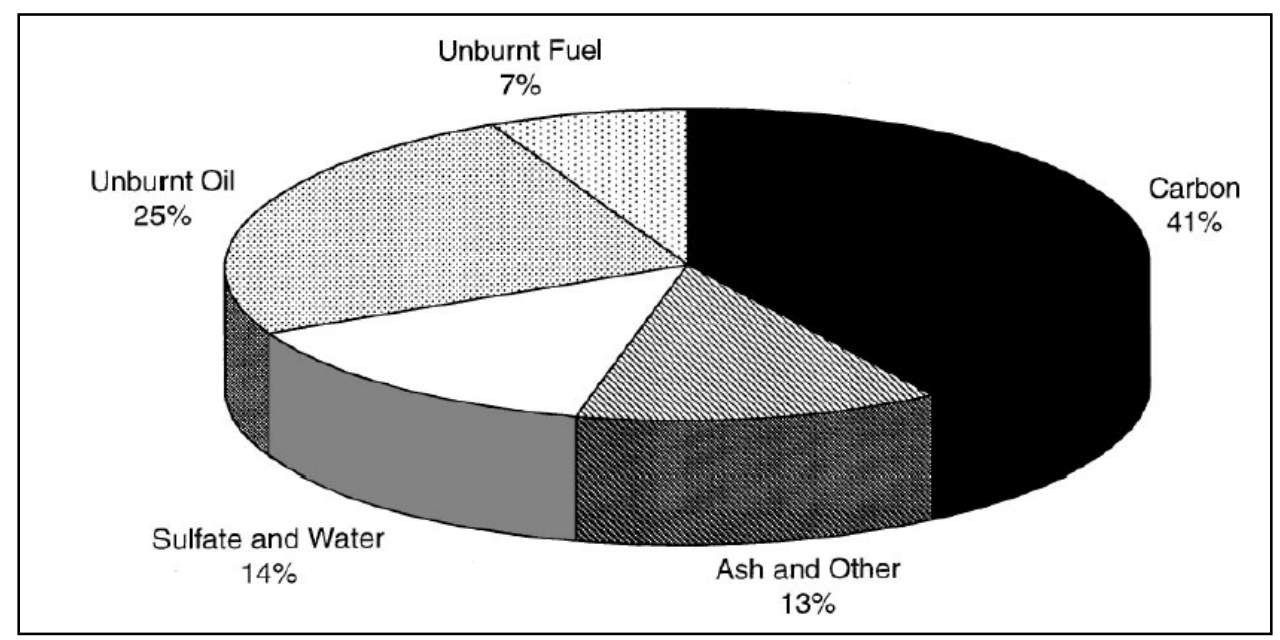

Figure 2-1 Typical composition of PM from a late 1990 diesel engine tested on a FTP cycle (Kittelson, 1998b).

Current model year natural gas engines employ spark ignition while operating on a stoichiometric air/fuel ratio strategy. This path has been primarily adopted to employ a threeway catalyst to control NOx emissions. Since, natural gas engines utilize a gaseous fuel, the resulting combustion is soot free. PM emissions from natural gas engines completely consist of organic carbon in nature (Lev-On et al., 2002, Ayala et al., 2002). However, the use of an oxidation catalyst has been shown to reduce the concentrations of organic carbon by removal of VOC (volatile organic compounds) (Ayala et al., 2003). Due to the absence of elemental carbon in the exhaust of natural gas, PM nanoparticle formation is highly dependent on dilution conditions and availability of nucleation precursors. In the presence of an oxidation catalyst, the concentrations of hydrocarbons that could nucleate to form a particle are reduced, and hence inhibit gas to particle conversion. However, under certain operating conditions lubricating oil proves to be a significant contributor to nanoparticle formation (Kittelson et al., 2008). A study conducted at WVU on a single cylinder research engine fueled by hydrogen showed oil 
consumption to be well correlated with engine operating parameters such as intake air boost, engine speed and in-cylinder pressures (George, 2008). The lube oil derived PM is more noticeable in heavy-duty natural gas engines, due to the fact that natural gas combustion is relatively soot free, and vehicles primarily operate without a DPF. The influence of lubrication oil on PM emissions is more observable as it often contributes to significant mass emissions from these soot free engines.

\subsection{EVOLUTION OF PM EMISSIONS}

\subsubsection{ENGINE EVOLUTION}

Heavy-duty diesel and alternative fueled engines have evolved from a primitive technology to a current advanced sensor based control systems. The thrust behind this transformation has been the moving target of achieving stringent emissions standards. Two criteria pollutants that have been progressively regulated have been NOx and PM. Due to the trade-off that exists between these two pollutants it is natural to observe an increase in engine out PM, while attempting to reduce engine out NOx. Manufacturers have adopted various strategies to alleviate the PM increase as a result of NOx reduction strategies such as EGR. One of the major developments in this regards has been the advancement in fuel injection technology and strategies that use high pressure direct injection and injection rate shaping to improve combustion performance and reduce the mass of engine out PM (Ardanese, 2008).

Advanced diesel engines have various electronic control devices that work together in achieving a targeted emissions goal. These emissions control devices such as VGT, EGR valve and high-pressure fuel injectors are optimized for optimum in-cylinder combustion. The strategies associated with each device independently affect the in-cylinder combustion in various forms and in turn directly affect the PM formation mechanism. In a study conducted at West Virginia University it was observed that VGT positions associated with high boost pressures resulted in lower soot production and smaller accumulation mode particles (Ardanese, 2008). The study showed that by varying the VGT position from $26 \%$ to $30 \%$ the primary particle diameter in the accumulation region decrease from $89 \mathrm{~nm}$ to $53 \mathrm{~nm}$. Further the study also showed that increase in EGR levels, resulted in a direct increase in particle 
diameters (Ardanese, 2008). Figure 2-2 and Figure 2-3 show the effect of VGT and EGR valve position on primary particle diameter from an advanced control diesel engine. Many of the improvements to diesel engine technology have contributed to lower mass of PM and smaller diameter primary combustion generated nanoparticles.

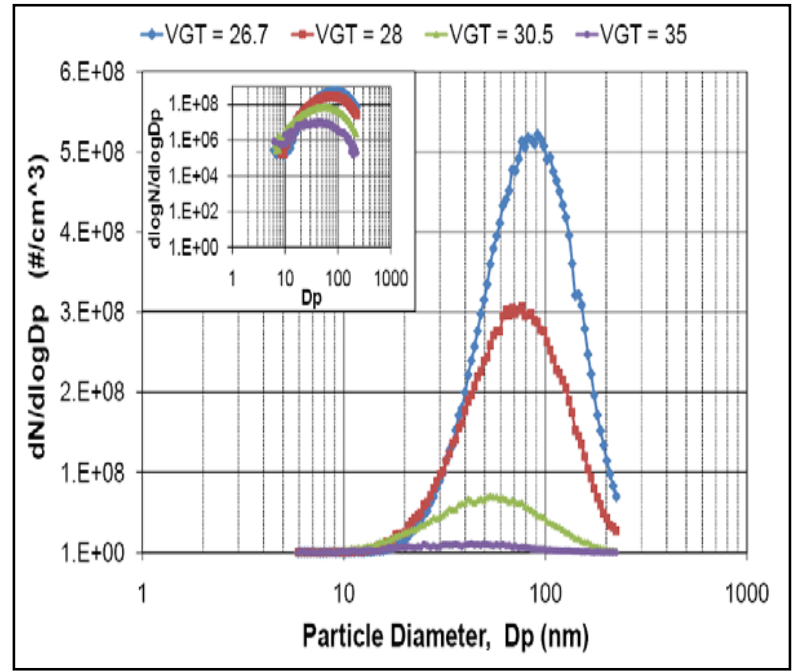

Figure 2-2 Effect of VGT on engine out particle size distribution from diesel engine and concentration (Ardanese, 2008)

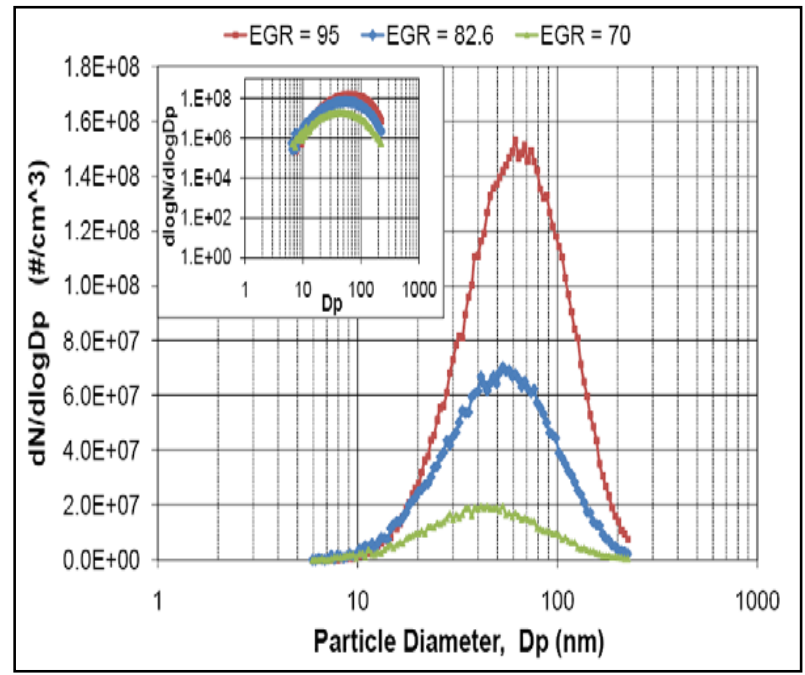

Figure 2-3 Effect of EGR valve position on engine out particle size distribution from diesel engine and concentration (Ardanese, 2008)

Similarly, it can be expected that PM from stoichiometric natural gas engines to have reduced significantly in comparison to PM mass emissions observed from early model year lean burn engines. Early, lean-burn natural gas engines faced numerous design challenges, which affected the durability of the engines. This resulted in higher mass emissions of PM due to a pronounced effect from the lubrication oil (Thiruvengadam et al., 2010). Early lean-burn engines operated without an oxidation catalyst, however growing concerns over CO and nomethane hydrocarbons (NMHC) emissions, forced regulatory agencies to mandate retrofit of natural gas vehicles with oxidation catalysts. Lean-burn engines inherently faced temperature limitations that affected oxidation catalyst activity at certain operating conditions and hence resulted in higher VOC content in the exhaust, which in turn increased organic carbon (OC) emissions. In comparison, an advanced stoichiometric fueled natural gas engine, that operates at higher exhaust temperatures than its lean-burn counterpart are characterized by ultra-low 
level PM mass emissions. However, lubrication oil based PM, in the form of ultrafine and nanoparticle emissions is still a significant source of PM that does not always contribute to significant emission mass, but results in increased number count emissions.

\subsubsection{FUEL EVOLUTION}

Beginning 2007 fuel sulfur levels have progressed from a low sulfur level of 500 ppm (wt.) to ultra-low sulfur level of below 15 ppm (wt.). The impetus for this change has been to protect catalytic after-treatment systems from sulfur poisoning. However with fuel sulfur content below 15 ppm (wt.), nanoparticle formation at high temperature operating conditions of the engine has been reported in the literature (Kittelson et al., 2008, Thiruvengadam et al., 2012). Sulfur based PM emissions are usually in the form of sulfates or sulfuric acid droplets, a result of sulfur trioxide mixing with water vapor in dilution air. In modern diesel engines, urea based SCR systems play a major role in sulfate formation. Aqueous urea injection introduces ammonia in the exhaust which could react with oxides of sulfur under certain exhaust temperature conditions to form ammonium sulfate crystals (Majewski, 2005), which in turn could contribute to PM emissions.

\subsubsection{LUBRICATION OIL INFLUENCE ON PM EMISSIONS}

Lubrication oil is a significant contributor to PM emissions from internal combustion engines. In non DPF equipped diesel engines, the contribution of lubrication oil to PM is masked by in general higher fuel sourced. In the presence of a DPF the total mass emissions of PM is most often below detection limits. The contribution of lubrication oil is well observed in natural gas engines as the gaseous fuel combusts relatively soot free and hence the need for a particulate trap is not justified.

Lubrication oil's contribution to PM emissions is directly related to the durability of engine components that present the pathways for the entrainment of lubrication oil into the combustion chamber either through the intake manifolds or via the piston rings or directly into the exhaust stream through the turbochargers. Oil entrainment into the combustion chamber could occur through three mechanisms a) oil seeping through the piston rings b) evaporation of oil deposited on the walls of the cylinder, and c) oil mist as a result of crank case scavenging 
into the intake manifold. Of the three mechanisms evaporation of oil from the cylinder walls and closed crank case ventillation is inevitable. However, well designed oil traps could minimize the effect of oil contribution due to crankcase scavenging. The oil entrainment into the combustion chamber through piston ring is significant during low load engine operating conditions characterized by lower in-cylinder pressures resulting in insufficient sealing of the combustion chamber from the crank case by the piston rings (Nakashima et al., 1995).

Lubrication oil contributes to a significant amount of soluble organic fraction (SOF) in to the PM composition. Low temperature combustion gases during the exhaust stroke may evaporate the oil transported onto the cylinder walls through the piston rings resulting in incomplete oxidation of the oil contributing to increased SOF content in the exhaust. A study has reported close to $30 \%$ of lubrication oil evaporation occurs during the late part of the exhaust and expansion stroke (Froelund et al., 2001). Most literatures report oil consumption mechanisms only in spark ignited engines and light-duty diesel engines. However, heavy-duty diesel engines with higher compression ratios, will result in lesser lube oil transported through piston rings would be, lesser in comparison to SI engines. Also, the design of the in-cylinder components are more robust, which aid in better oil control even during low load operating conditions. And the result is lower oil entrainment into the combustion chamber. The type of vehicle operation plays a vital role in the rate of oil entrainment into the combustion chamber. Vehicle operation characterized by frequent stop-and-go and extended idle periods could result in higher lubrication oil consumption. This is based on the findings that idle operation result in lower incylinder pressures and therefore would result in increased oil consumption (Anderson et al., 2004). Since current natural gas engines operate on a SI engine platform, lubrication oil consumption mechanisms would be similar to those reported by Anderson et al. (Anderson et al., 2004). Further, Zielinska et al. have shown that PAH emissions in gasoline engines, closely resemble PAH composition of used lubrication oil and not fuel (Zielinska et al., 2004). Results from this study can be used to infer that used oil contribution to PM in natural gas engines would be more significant than the contribution of fresh oil. The observed change in oil consumption rate between used and fresh oil indicate that the viscosity of oil to change with engine operating hours. 
Lubrication oil also results in several inorganic constituents that form a part of the total PM emissions from internal combustion engine. Lubrication oil consists of various additives that provide detergents, dispersant, acid neutralizers, anti-oxidants, corrosion inhibitors, and antiwear properties to the lubrication system. Hence, as a result of lubrication oil consumption, these additives will manifest themselves as inorganic constituents in the exhaust. Some of the predominant elements observed in engine exhaust and directly related to lubrication oil are $\mathrm{Ca}$, Zn, Ph and S (Fujita et al., 2007). Sulfate emissions are a major source due to the lubrication oil sulfur content that is subject to partial oxidation inside the combustion chamber. A report from Desert Research Institute (DRI), documented the chemical analysis of over 10 different lubrication oils over varying oil life. The study observed an average oil sulfur composition of 3000 microgram/gm, indicating a significant source of sulfur for the formation of sulfate ions (Fujita et al., 2006). Findings from this report suggests a significant contribution of lubrication oil towards the formation of sulfate compounds.

The contribution of lubrication oil to PM has been a widely researched topic. However, the fractional contribution of lubrication oil to the volatile and non-volatile fraction of PM and its subsequent health effects has not been clearly established. While studying PM composition from natural gas engines, it is possible to observe the effects of lubrication oil sulfur in PM formation under real-world operating conditions with better clarity due to the absence of the contribution from fuel sulfur. As pointed out earlier in this section, lubrication oil consumption between heavy-duty $\mathrm{SI}$ and compression ignition $(\mathrm{Cl})$ engines is vastly different. However PM mass fraction analysis derived from SI engines could be used to explain mass emissions of organic and inorganic PM fractions during certain operating conditions of heavy-duty diesel engines. Further, the objectives of the current study involving the characterization of the different compositions of PM and correlating their toxicity effects will provide a holistic approach in identifying the source of the emission and suggesting a direct relation between source and end effect.

\subsubsection{AFTER-TREATMENT SYSTEM INFLUENCE ON PM EMISSIONS}

The variety of after-treatment systems employed to meet regulations has contributed to changes in composition and emission rates of PM. Subhasis et al. concludes that after- 
treatment systems altering the physical and chemical characteristics of PM and induce changes in particle formation pathways (Subhasis et al., 2009).

Diesel particulate filters have been identified as the only after treatment system that could help achieve the current PM emissions standard of $0.01 \mathrm{gms} / \mathrm{bhp}-\mathrm{hr}$. DPFs have demonstrated PM mass filtration efficiencies close to $99 \%$ over most operating conditions. However, many studies have also reported the increased emissions of nucleation mode particles downstream of a DPF (Herner et al., 2011, Thiruvengadam et al., 2012, Giechaskiel et al., 2007, Kittelson et al., 2008). It is to be noted that the emissions of nucleation mode particles downstream of a DPF, are not observable throughout the entire operating regime of the engines. Nanoparticle emissions downstream of a DPF are in fact most often a function of exhaust dilution conditions and exhaust temperature. As, DPFs are either catalyzed for passive regeneration or noncatalyzed and are equipped with fuel injectors for active regeneration, high temperature engine activity contributes to a fractional loss in filtration efficiencies of a passively regenerating trap and possible increased hydrocarbon emissions during active regeneration events. PM emissions downstream in an after-treatment system are entirely dependent on engine operating conditions. A recent study by Biswas et al. has shown the size segregated PM emissions compared between baseline engine configuration and 6 different after-treatment systems that included catalyzed DPFs and SCR systems (Biswas et al., 2009b). The study presented data from two different vehicle operations namely cruise mode and UDDS cycle. The results of the study indicated that during cruise mode operation, PM emissions downstream of most aftertreatment systems are in the nucleation region and dominated by significant sulfate concentrations. The study also reported that the vanadium based SCR system, exhibited highest sulfate concentration over the UDDS cycle, hence suggesting a lower catalytic activity temperature and higher conversion rate of $\mathrm{SO}_{2}$ to sulfates (Biswas et al., 2009b). Although the referenced study does not, document the source of sulfur for sulfate formation, the results presented are well correlated with other literature that has attributed the formation of sulfates to lubrication oil more than fuel sulfur. Also, cold start conditions with no catalytic activity have been attributed to increased nanoparticle emissions from a DPF equipped diesel vehicle (Giechaskiel et al., 2007). 
Given the fact that the DPF filters all elemental fractions, PM composition downstream of a DPF will be dominated by OC. While characterizing the OC emissions downstream of a catalyzed after-treatment system, it is also important to assess the levels of water soluble organic compound (WSOC) fraction compared to the total OC concentrations. WSOC are indicative of aerosol aging and can be viewed as a secondary PM formation pathway and not necessarily a primary OC fraction. WSOC are usually formed by the coagulation of gas phase partitioning of certain organic fractions such as formic acid, nitro-PAH and aliphatic compounds (Asa-Awuku, 2008). The study by Biswas et al. illustrates that the low WSOC/OC ratio from baseline vehicles is indicative of a freshly formed primary organic PM. Also higher WSOC/OC ratios downstream of catalyzed after-treatment system, indicates a higher water solubility of $\mathrm{OC}$ in the nucleation region and catalyzed after-treatment systems contribute to possible precursors for WSOC formation (Biswas et al., 2009b).

While comparing the exhaust constituents of current advanced diesel engines and stoichiometric natural gas engines, it can be seen that tailpipe concentrations of nucleation mode precursors and the levels of elemental carbon are quite similar. Although, CNG exhaust is characterized by higher total hydrocarbon content, it almost entirely consists of methane which does not contribute to nanoparticle formation. The one main difference in the two cases is the effect of fuel sulfur and its subsequent oxidation processes which has possibilities of sulfuric acid based particle formation at exhaust temperatures greater than 380 Deg C (Thiruvengadam et al., 2012) . Consequently in order to isolate the effects of fuel based sulfur and focus our attention on discerning the toxicity of volatile and non-volatile particles from heavy-duty engines as result of combustion and lubrication oil consumption, it would be appropriate to study PM profile from CNG exhaust and further apply the findings to diesel engine exhaust.

Another recent advancement in after-treatment technology has been the use of urea based SCR systems for NOx abatement. Aqueous ammonia which is used as a reducing agent for NOx control, is believed to alter particle formation mechanisms to aid in the formation of ammonium sulfate and ammonium nitrates as result of byproducts of SCR reaction (Herner et al., 2011). However, the contribution of SCR systems to particle toxicity has not been well documented yet. Similarly, in the case of stoichiometric CNG engines, the use of TWC as a NOx 
control after-treatment system have been shown to result in significant levels of ammonia as result of high temperature TWC activity. Hence, there is a possibility that a similar particle formation mechanism may be observed from both SCR equipped diesel and TWC equipped natural gas engines.

Metals have been identified as one of the major fractions of PM from advanced heavy-duty engines (Verma et al., 2010). The source of metallic emissions can be traced to lube oil consumption and catalytic after-treatment systems that continuously age with vehicle. With increasing catalytic systems in the exhaust stream, metallic emissions could constitute a major fraction of tailpipe PM emissions from heavy-duty engines, and could further bias toxicity results of the non-volatile fraction of PM. Hence, it is imperative to discern accurately the composition of non-volatile fraction of PM to better understand the relative contribution of EC and non-organic fractions to toxicity.

\subsection{HEALTH EFFECTS OF PM}

Regulatory agencies have aimed at reducing PM emissions due to concerns about potential PM related health effects suggested by toxicological studies. Published literature has shown the increased morbidity and mortality in people exposed to ultrafine particulate matter emissions (Dockery et al., 1993, Pope III et al., 1995, Englert, 2004). Although most exposure studies are conducted for ambient PM, it is to be noted that a major anthropogenic source for ultrafine PM in the atmosphere is from mobile sources and specifically that of the heavy-duty vehicle population. Hence urban population exposure to heavy-duty vehicle PM could be a significant driving force for deterioration in human health. Diesel exhaust in particular has been identified as a potent carcinogen $(1998,2012)$. Most carcinogenic species in diesel exhaust appear in gas phase, but these gas phase organic compounds do act as precursors for nanoparticle formation. One of the main setbacks in existing literature has been the absence of a size segregated volatility measurement with its associated health effects. The absence of literature can be justified by the fact that smaller particles have negligible mass and most toxicological experiments require considerable aerosol mass to obtain a discernible biological response. The problems are compounded while trying to simulate extended dosage and exposure (as in real 
world interactions of humans with PM) within the confines of an experimental setup bounded by test procedures and time limitations.

A number of factors such as particle number, size, surface area, chemical composition, morphology and deposition fraction are likely to influence the toxicity of nanoparticles. All of these factors are directly dependent on engine operating conditions and exhaust configurations. Also, of significant importance is the composition of PM nanoparticles emitted from heavy-duty internal combustion engines. All combustion generated particles are considered to exist in equilibrium between two states i.e gas phase and solid phase. Particles are viewed to be comprised of solid core with low volatile and semi-volatile materials adsorbed on this core. This view can be challenged by the fact that homogenous nucleation produces particles completely volatile in nature formed as a result of condensing vapors within regions of super saturation conditions of VOCs. However, homogenous nucleation is a highly uncertain phenomenon governed by various factors such as dilution conditions and partial vapor pressures of the various VOCs. Also, homogenous nucleation can be considered as an atmospheric governed particle formation mechanism as opposed to primary particle formation from heavy-duty vehicles that are completely governed by engine operation and exhaust conditions. Hence, heterogeneous nucleation of gas phase compounds condensing on a solid core is believed to be a more dominant particle formation mechanism during the cooling of heavy-duty vehicle exhaust. Moreover, binary nucleation of $\mathrm{SO}_{3}$ with water to produce sulfuric acid based particles has recently been identified as a dominant particle formation mechanisms from heavy-duty engines operating at certain high exhaust temperatures (Vaaraslahti et al., 2005).

A few studies have measured the volatility of PM in dynamometer tests using tandem differential mobility analyzer (TDMA) systems (Sakurai et al., 2003). The volatility measurements yield information on particle mixing characteristics and nanoparticle vapor pressures, which can be compared with standards to estimate the carbon numbers of the volatile organic components. Given that inhalation and deposition behavior of nanoparticles is determined by particle mobility rather than aerodynamic properties, mobility based 
measurements are especially well-suited to use with nanoparticle studies related to health effects.

The current knowledge base woefully lacks data that directly links the physical and chemical characteristics of specific exhaust emissions species, or a combination of species to health risks, and how such health risks change as a function of changing composition of exhaust PM emissions. Of the studies that have been conducted previously focusing on discerning the toxicity effects of PM, a few important ones are discussed here.

a) Study by Liu et al. measured genotoxic activity on solvent extracts from diesel and gasoline engine PM. The study indicated distance specific mutagenic activity of diesel PM to be 3-7 times higher than that of gasoline exhaust PM. However mutagenicity activity from semi-volatile organic compound (SVOC) extracts from diesel and gasoline indicated gasoline SVOC to be more potent on a mass basis (Liu et al., 2005).

b) Gene mutation activity from organic solvent extracts of diesel PM from a single cylinder diesel engine was studied by McMillian et al. The study investigated the effects of various fuels such as Fischer Tropsch, natural gas, and conventional diesel on PM toxicity. The study revealed a significant difference in mutagenic activity between different fuel types as a result of different organic fractions in PM (McMillian et al., 2002). One of the salient outcomes of this study was the development of a quadratic surface dose response relationship between brake mean effective pressures (BMEP), engine speed and toxicity response. This is one of the few studies that specifically correlated engine operating conditions to toxicity response.

c) Of the studies that focused on fuel and after-treatment systems, the work of Bagely et al. is worth mentioning. The researchers found that the use of an oxidation catalyst in combination with a PM trap substantially decreased the particle number concentration and mutagenic activity of PM emissions from gasoline engines using leaded and unleaded fuel (Bagely et al., 1996). 
d) A study by Seagrave et al. can be considered as one of the most important and comprehensive work with respect to toxicity and mutagencity of particulate matter from heavy-duty natural gas vehicles. The authors sampled particulate matter and vapor-phase SVOC from natural gas transit buses with different PM emissions rates such as high emitter, normal emitter and a new technology with oxidation catalyst. Results indicated mutagenicity was highest for the high emitter vehicle and lowest for the new technology vehicle. The study also concluded that mutagenic potencies for the CNG PM samples were similar in potency level observed by gasoline and diesel PM (Seagrave et al., 2005).

The studies listed above are all characterized by certain limitations with respect to experimental conditions that were adopted and the engine technologies the studies represent. Almost all toxicological studies have been carried out as engine dynamometer experiments with engine technology that may not necessarily represent current model year technology. Also, engine load conditions with the exception of the study by McMillian et al (McMillian et al., 2002) are usually characterized by steady state operating conditions, which do not completely represent real world engine operation and subsequent PM formation. Natural gas engine studies cited herein have all been carried out on oxidation catalyst equipped lean burn natural gas vehicles, which are no longer representative of current model year technology of CNG vehicles. The current study presented here attempts to investigate in particular toxicity effects from advanced stoichiometric natural gas heavy-duty vehicles, which have emissions rates similar and in some operations lower than DPF-SCR equipped modern diesel engines.

Since the composition of PM will vary in accordance with treatment method of the sample or the vehicle's exhaust, this study will adopt the definition of PM as per USEPA's operational definition "of all mass collected on a filter from a diluted exhaust stream at a temperature of $47 \pm 5$ Deg C". Since, the PM standards of heavy-duty vehicles are subjected to this definition it is imperative to understand the fractional toxicity effects of PM collected in accordance with this operational definition.

PM formation from heavy-duty vehicle exhaust depends on the type of fuel, engine operating loads and after-treatment systems. Hence, composition of PM can be viewed as 
engine duty cycle dependent. Population exposed to urban traffic and near highway traffic will have PM exposure patterns completely different from each other. This can be attributed to the fact that, within the urban scenario, heavy-duty vehicles will be characterized by slower speeds, and lower loads and frequent acceleration and decelerations. On the contrary highway operations would be characterized by a more steady state operation with higher engine loads. As a result vehicle operation within an urban setup could be characterized by lower exhaust temperatures and in turn lower catalytic activity. In the case of stoichiometric natural gas engines, the temperature difference between highway and urban operation would be very similar, however, larger deviations in the closed loop air-fuel ratio control would exist due to the highly transient operations. A study conducted by Li et al. showed the differences in proinflammatory responses from particles sampled over two driving cycles namely, UDDS and the idle mode. The results of the study indicated a higher level of redox active metals and organic compounds over the UDDS cycle compared to the idle mode that contributed to oxidative stress in human aortic endothelial cells (Li et al., 2010).

With the advent of modern emission control technologies in heavy-duty vehicles in particular diesel vehicles, the balance between EC/OC emissions is continuously changing with respect to engine operation and after-treatment system state. Periodic DPF regeneration (active or passive) result in momentary loss in filter efficiency and would result in higher EC emissions. Similarly in natural gas engines, highly transient activity would result in frequent rich fuel-air mixture to cater to the power demands of the driver, thereby resulting in possible increased VOC composition contributing to OC emissions. Elemental carbon or black carbon has been associated with various respiratory and cardiovascular disorders. A controlled human exposure study has indicated a close relationship between indoor and outdoor black carbon levels to cases of respiratory conditions such as asthma. However, the same study disproves its hypothesis suggesting a strong association of black carbon with adverse cardio vascular effects such as blood pressure and pulse rate (Jansen et al., 2005). The findings from the study by Jansen et al. could be related to the differences in deposition factors in the various regions of the human airway, hence contributing to one response and not the other. Details of deposition fraction and surface area will be discussed in the following sections. 
Most studies that elucidate the link between respiratory conditions and elemental carbon concentration represent human population exposed to ambient levels of elemental carbon. Hence, an accurate dosage vs. response is not available for a specific source of elemental carbon such as heavy-duty vehicle population. In the presence of exhaust particulate filters it is uncertain to what extent tailpipe concentrations of elemental carbon could be attributed to adverse health effects. Also, it is important to understand, the levels of exposure that would exhibit a significant response from toxicity indicators and to further matching those levels of elemental carbon emissions to its respective engine operating conditions such as transient, highway or idle. This would provide the accurate link between engine operation and toxicity, rather than generalizing that all vehicle activity will produce PM emissions that are potent to the health of humans.

Literature has documented the importance of WSOC in affecting the hygroscopicity of the aerosols wherein it enhances the possibility of PM to act as cloud condensation nuclei. WSOCs primarily consist of dicarboxylic acids, aliphatic amines, compounds consisting of carboxyl groups and carbonyl species (Bao et al., 2009, Biswas et al., 2009b). It is commonly believed that anthropogenic emissions of particle phase WSOC compounds is not common, and on the contrary WSOC fraction in OC increase with continuous aging of the aerosol (Bao et al., 2009). Hence, WSOC fraction in PM can be viewed as a secondary particulate matter formation pathway pertaining to the atmospheric reactions. A study by Biswas et al. suggests a strong association of WSOC to increased oxidative potential of PM (Biswas et al., 2009a). The study suggests that changes in oxidative potential could be attributed to chemical changes induced by catalytic after-treatment systems in the exhaust stream. Literature has pointed to the role of WSOC in increasing the acidity of organic PM composition (Bao et al., 2009). Semi-volatile species such as oxygenated PAHs, nitro-PAHs and quinines have exhibited high correlation towards certain bio assays, which indicate the oxidative potential of a species (Biswas et al., 2009a, Biswas et al., 2009b).

Metallic fractions in PM originate from various sources in the exhaust of a heavy-duty vehicle. Traditionally in a baseline engine configuration, metals emissions were entirely due to engine wear issues and lubrication oil consumption. However, with the use of multiple after- 
treatment systems that are catalyzed for purposes of emissions control, metallic content in PM has also increased. PM toxicity associated with metals is highly proportional to their mass fractions in the total PM mass. A study by Verma et al. has observed significant changes in toxicity responses in the presence of transition metals in total PM composition (Verma et al., 2010). Redox active transition metals such as $\mathrm{Fe}, \mathrm{V}, \mathrm{Ni}, \mathrm{Cr}, \mathrm{Cu}$ and $\mathrm{Mn}$ are observed to be more in abundance in exhaust of after-treatment system equipped heavy-duty diesel engines in comparison to baseline diesel (Verma et al., 2010). The study found that vehicles equipped with retrofit after-treatment devices, are characterized by higher metallic fraction in PM, and have greater tendency to induce oxidative stress in living cells (Verma et al., 2010) . In another study, Distefano et al. have indicated the direct contribution of copper ions in generation of reactive oxygen species (ROS) through a chemical toxicity assay procedure. The study further indicates the role of certain transition metals present in PM emissions from diesel engines that could alter the anti-oxidation properties of cells (Distefano et al., 2009).

This section, detailing the health effects of PM has examined various studies that have attempted to correlate different PM compositions to its toxicity. Literature has also related driving cycle dependency on PM composition and to its toxicity response. However, the link between engine operating conditions to toxicity is woefully lacking. Driving cycle based differences such as distance, speed, time and PM mass will not be sufficient enough to derive broad conclusions about toxicity effects of heavy-duty PM with respect to vehicle operation. However, an in depth analysis of factors such as lubrication oil consumption, average exhaust temperatures, after-treatment performance, durability, inorganic emissions, nucleation and accumulation mode particle concentrations are necessary to define characteristics of engine operation that can be related to PM composition and subsequent toxicity potential. Current ultra-low engine-out PM concentrations have induced a large uncertainty in mass measurement procedures, consequently imparting a large uncertainty in the measured toxicity also. Discerning toxicity response from ultra-low level PM emission rates is a challenging task. This sampling method adopted in the current work has attempted to improve sample loading on filter media through a process of high volume sampling in order reduce test times and possible sampling artifacts due to extended sample collection time.

20 | P a g e 


\subsection{TOXICITY ASSAYS}

Toxicity assays can be broadly divided into two categories, namely 1 ) In vitro and 2) In vivo studies. In-vitro refers to toxicity analysis performed to discern the toxic effects of a certain compound using cultured bacteria or mammalian cells. And In vivo testing refers to evaluation of toxic potential of a compound using live animals or humans. There are different approaches and surfactants that can be used to perform in vitro studies. A few important in vitro approaches in reference to diesel PM are: 1) Researchers at National Institute of Occupational and Safety Hazard (NIOSH) tested three different surfactants namely Salmonella bacteria (Ames Salmonella Assay) and mammalian cells derived from Chinese hamster (Micronucleus (MN) Assay). The study was aimed at discerning toxicity potential by understanding bacterial mutagenicity using the Salmonella extracts and DNA damage in the mammalian cell extracts. One of the salient features of this study was that it challenged the conventional in vitro approach of introducing organic solvent extracted PM to cell cultures, by introducing a method of dosing wherein diesel particulate matter is directly, in its true form is introduced in to the surfactant. The researchers believed that this method in comparison to extraction methods, better illustrates the possible effects of particle morphology and chemical structure to its toxicity potential (Shi et al., 2005).

2) Another study conducted by researchers at University of California, Los Angeles used human aortic endothelial cells (HAEC) to study the influence of PM in increasing cardiovascular risks in humans. Diesel PM was collected on T60A20 glass fiber filters, and further extracted out of the filter using ultra pure water. The extract was later used to expose the HAEC to study its toxicity response (Li et al., 2009).

Although cell based In vitro studies can be considered as non-selective in their response to various components PM, the use of a particular cell or bacteria will indirectly bias the toxicity response to certain components of PM. However, by introducing the PM samples directly to the cell it becomes difficult to discern the composition of PM that influenced the toxicity response. Hence, a cell based in vitro study can be viewed as an indicator of the overall health effect of PM and not necessarily point to a specific composition of PM. If in case a compositionally selective toxicity response is desired then the particulate sampling method would have to be 
directed towards sampling individual compounds such as metals, organics and elemental carbon and individually subjecting the cell cultures to these samples.

Cell-free or chemical methods of performing In vitro studies have been widely researched. These methods do not employ any living cell cultures and on the contrary use chemical substances as indicators for toxicity response. Chemical based In vitro approach is highly selective in its response to different fractions of PM, and can alleviate some of the concerns identified with cell based studies. For example DTT assay is highly responsive to organic material in PM, DHBA assay is very responsive to fenton-active (reaction of certain metals with oxygen to produce highly reactive hydroxyl ions) metals such as $\mathrm{Cu}$ and Fe (Verma et al., 2010) .

From the literature it is clear that there exists no one unified metric to quantify toxicity of PM. Different assays quantify toxicity potential using different units or in this case metrics. Although, the metrics used to represent PN toxicity may vary, the basic underlying principle or objective of all assays are aimed towards discerning the oxidative stress induced in a cell or the oxidative potential (OP) of a compound that could possibly inflict damage to living cells. Oxidative stress is a result of generation of reactive oxygen species (ROS) to levels that would exceed the natural antioxidant defense level of a cell. Higher ROS content can damage proteins and DNA and cause death of cells. High ROS levels can also induce inflammatory responses that are cause for many respiratory and pulmonary diseases (HEI, 2010).

ROS are chemical species which consists of highly reactive oxygen species or superoxide. They include chemical species that contain $\mathrm{OH}$ radicals, singlet oxygen and hydrogen peroxide. ROS is a common byproduct of aerobic metabolism in human body. The production of antioxidants from cells, maintains the balance of ROS species, and in turn regulates the various cellular functions (Landreman et al., 2012). However, entry of foreign substances with toxic properties can increase the concentration of ROS compounds relative to the antioxidant production. This ability of a substance to induce ROS generation is called the OP of that substance. The cellular reaction aimed at neutralizing the excessively generated ROS, results in the oxidative stress of the cells. PM induced ROS generation can be studied through various cell based and cell free assays. 
Alveolar macrophage (ROS) assay is a method to measure toxicity response from alveolar macrophages exposed to aqueous suspension of particulate matter. This method represents a well correlated model to link pulmonary inflammation response to particulate matter concentration. This method is also highly sensitive to the presence of transition metal content in the PM sample (Verma et al., 2010).

The current study will present data from three different assays namely a) Dithiothreitol (DTT) assay b) Didydroxybenzoate (DHBA) assay and c) Macrophage ROS assay. The selectivity in response of these assays and documented findings in the literature with respect to heavyduty PM will be discussed in the subsequent sections. Keeping in line with the global objective of this study, literatures cited and its interpretation will not include a detailed analysis of the toxicity aspects. Instead, the toxicity response will merely be used as an indicator metric for adverse health effects. The study will however attempt to correlate engine based factors to PM composition and finally point towards the direction of its possible health effects if any. The absence of a single toxicity metric and the complex composition of PM make it challenging to comprehend the health effects of heavy-duty PM as a whole. As in previous cases, the study will provide insights to possible links between different compositions of PM and its ability to induce oxidative stress in cells.

\subsubsection{DTT ASSAY}

DTT assay is a type of a cell free assay where a chemical called dithiothreitol is used as an indicator of oxidative potential of a test sample. The assay uses the consumption of DTT as a marker to identify cellular redox reaction in the presence of oxygen (Verma, 2011). Hence, the rate at which DTT is consumed is proportional to the mass of redox active species present in the sample and consequently greater oxidative potential. Researchers have found that the DTT assay demonstrates selectivity towards organic compounds such as quinones, carbonyl, volatile fraction of particulate matter and PAH emissions. A study by Charrier and Anastasio has illustrated the response of the DTT assay towards the presence of transition metals in specific copper and manganese. The study further shows that up to $80 \%$ of DTT consumption can be attributed to the presence of transition metals in a sample consisting of mixture of transition metals and quinones (Charrier and Anastasio, 2012). Findings from another study has exhibited 
good correlation between DTT consumption of ambient PM with production of stress proteins from tissue cultures, linking the influence of organic fractions of PM towards cellular toxicity (Cho and Froines, 2006). Hence, the use of DTT assay in the current study is used to elucidate the difference in DTT activity between thermally denuded and non-denuded assays. The difference in DTT activity, if any, should directly indicate the contribution of volatile fraction to PM toxicity. The above referenced study has revealed that DTT activity correlated well with OC $\left(r^{2}=0.53\right)$ and with elemental carbon $\left(r^{2}=0.79\right)$ (Cho and Froines, 2006). It is to be noted that soot emissions in diesel engines does not comprise only EC. Soot in diesel engines is comprised of significant concentration of adsorbed PAH compounds. Higher consumption of DTT in the presence of PAH compounds and the possible higher fractions of PAH adsorbed on soot than in OC could have resulted in the better correlation of DTT activity towards EC. However, this scenario might be different for PM from natural gas engines, where the fuel is devoid of aromatic content and as a result lower in exhaust PAH content. The EC thus formed could be simply elemental carbon with minimal to no PAHs adsorbed. However, establishing a link between lube oil consumption and PAH emissions could provide insight of possible PAH fractions adsorbed onto EC. The current study will attempt to correlate PAH emissions, EC and OC concentrations to DTT activity. Hence, the denuded PM sample will effectively indicate DTT activity of predominantly EC and the non-denuded sample will be indicative of a DTT activity representative of $\mathrm{OC}$ and $\mathrm{EC}$ concentration. The difference in activity should effectively indicate the activity of OC fraction alone.

\subsubsection{DHBA ASSAY}

DHBA assay is a cell free assay that uses the production of dihydroxybenzoic acid as marker for in vivo toxicity. DHBA assay is highly selective towards the presence of transition metals in the filter sample. The basic principle behind the DHBA assay is based on the fact that derivatives of salicylic acid are present in human body as anti-inflammatory agents. And it is found that in the presence of salicylates, transition metals undergo Fenton's reaction to produce $\mathrm{OH}$ radicals (Coudray and Favier, 2000). Further, the reaction between salicylates and $\mathrm{OH}$ radicals produce DHBA. Hence, by quantifying DHBA levels, one can conclude the rate of $\mathrm{OH}$ radical production, which in turn can be viewed as the extent of salicylate production due to 
inflammatory response in the human body. The DHBA analysis is performed using highperformance liquid chromatography techniques. The work by Distefano et al. describes the development of this assay and illustrates the selectivity of this assay to metal content in PM. The research work documented the role of copper and iron in DHBA formation, and further elucidated the positive response of the assay to copper and other transition metals, while it correlated poorly with iron concentrations. Copper concentrations observed in diesel and ambient PM presented a good correlation $\left(r^{2}=0.95\right)$ with DHBA formation rate as shown in Figure 2-4 (Distefano et al., 2009). One of the drawbacks of the assay is the possible masking of DHBA formation due to other metals in the presence of higher concentration of copper. It has also been studied that chelation process (removal of metal from sample) of the sample completely suppresses DHBA activity, lending support to the hypothesis of high metal selectivity of the DHBA assay (Cho and Froines, 2006).

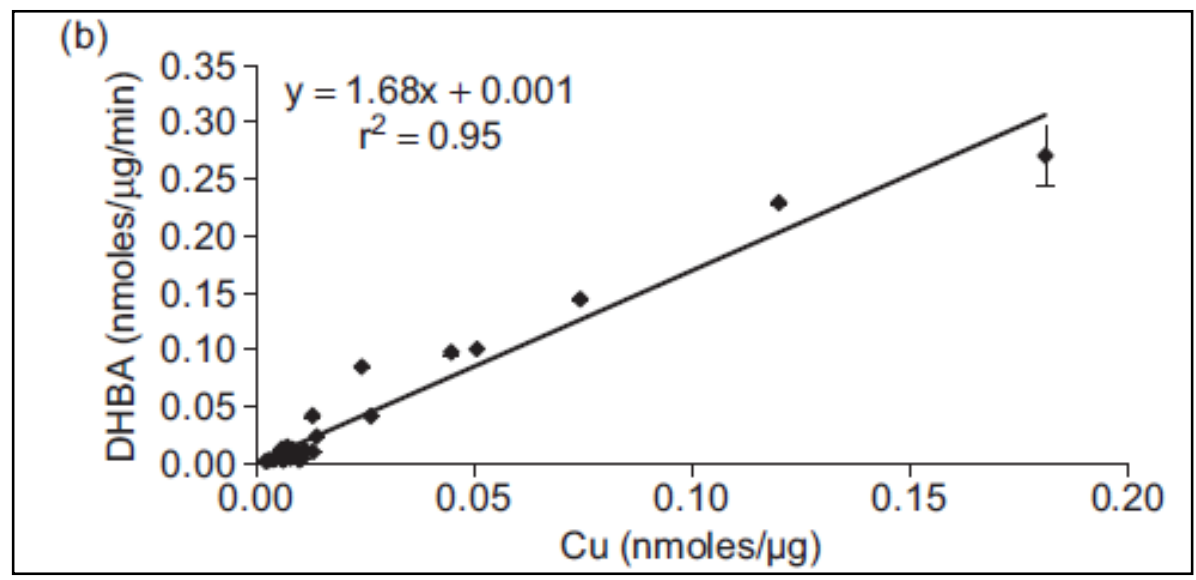

Figure 2-4 Correlation of copper concentration in ambient and diesel PM to DHBA formation rate (Distefano et al., 2009).

Internal combustion engines generated PM is characterized by a varied metals composition in PM since metals emissions from engines are due to a combination of factors involving engine operation and durability factors. As explained in 2.2.3 and 2.2.4 source of metals emissions could be from lubrication oil and or after-treatment systems. Not many studies have documented the DHBA activity of internal combustion generated PM. This lack of literary knowledge could be owed to the reasoning that DHBA assay has been a relatively new method developed in identifying oxidative stress in cells that are induced by the presence of metal composition through a cell free assay. Hence, the results provided in this work would 
provide an understanding of metal composition in total PM to DHBA activity. The solid fraction of PM, will consists of a mixture of EC, metals and inorganic ions, hence the correlation of mass fraction of metals in the PM sample to DHBA activity, will illustrate the oxidative potential of the PM due to its metals composition alone. The changes in mass fraction of metals in PM as a function of engine operating parameters will provide information on engine load characteristics that would possibly contribute to higher metal emission and, as a result, prove more potent to oxidative stress. This would help to reach conclusions on the type of exposure by humans (near highway or urban traffic) resulting in adverse health effects.

\subsubsection{ALVEOLAR MACROPHAGE ROS ASSAY}

Alveolar macrophage assay is a cell based toxicity assay wherein the aqueous PM samples will be introduced into rat alveolar macrophage tissues. Alveolar macrophages are commonly found in the inner epithelial cells of the airways and lungs and hence by quantifying ROS generation within alveolar tissues, would be an indicator of pulmonary inflammatory responses to PM (Verma et al., 2010). The assay uses 2,7-dichlorodihydrofluorescein diacetate (DCFH-DA) compound's reactivity with ROS compounds as a marker for cellular oxidative stress. The reaction of (DCFH-DA) with ROS compounds yields a highly fluorescent byproduct, which is measured using a fluorescent probe or plate reader. A compound called zymosan is used as the positive control, and the ROS response of a substance is presented as a function of zymosan equivalent response or zymosan units. The fluorescence induced by aqueous suspension of PM will be represented as equivalent fluorescence exhibited by the control substance, zymosan. A recent study by Landerman et al. has shown that this assay is effective for PM mass loading of less than 100 micrograms and that it accurately represents pulmonary response to PM exposure, as the lung airways are the first line of defense for the human body against particulate matter ingestion (Landreman et al., 2012).

Although the macrophage ROS assay is designed to exhibit the net effect of total PM, recent studies by $\mathrm{Hu}$ et al. and Verma et al. have observed the selectivity of this assay towards water soluble transition metals (Hu et al., 2008, Verma et al., 2009). Results from a recent study conducted by Verma et al. focused on PM emissions from retrofitted heavy-duty vehicles, suggests that ROS activity correlated well with concentrations of water soluble metals. The 
authors arrived at this conclusion by comparing ROS activity with and without removal of water soluble metals from the PM samples (chelation). The study showed that with removal of water soluble metals, the ROS activity was also lower for all driving cycles (Verma et al., 2010).

One of the limitations of existing studies documenting ROS production as result of PM exposure is the physical metric to which the toxicity response is normalized. Current works have represented toxicity responses with respect to physical mass of PM collected on filter. This representation would be accompanied by high variability, as gravimetric measurements of PM at ultra low level emissions are accompanied by high uncertainties. Also, if studies are aimed at discerning the toxicity effect of volatile fraction of PM, then the variability in mass could be orders of magnitude higher and lower of the mean value.

The following sections will study the possibility of alternate physical metrics such as surface area, lung deposited surface area (LDSA) and mass calculations using effective particle density to arrive at a metric that would correlate well with different observed size fractions of PM and their corresponding toxicity responses.

\subsection{PHYSICAL CHARACTERISTIC METRICS FOR PM ANALYSIS}

In regulatory terms, mass based metric has been widely accepted to be the physical metric that represents PM emissions from internal combustion engines. However, the mass based metrics fails to provide a definitive value at ultra-low level emissions of PM such as those from DPF equipped diesel and advanced natural gas engines. Figure 2-5 shows the typical particle number, mass and surface are distribution of diesel engine combustion generated particles having an aerodynamic diameter in the size range of $2 \mathrm{~nm}-200 \mathrm{~nm}$. With advanced aftertreatment systems, the higher particle diameters are most often not observed. 


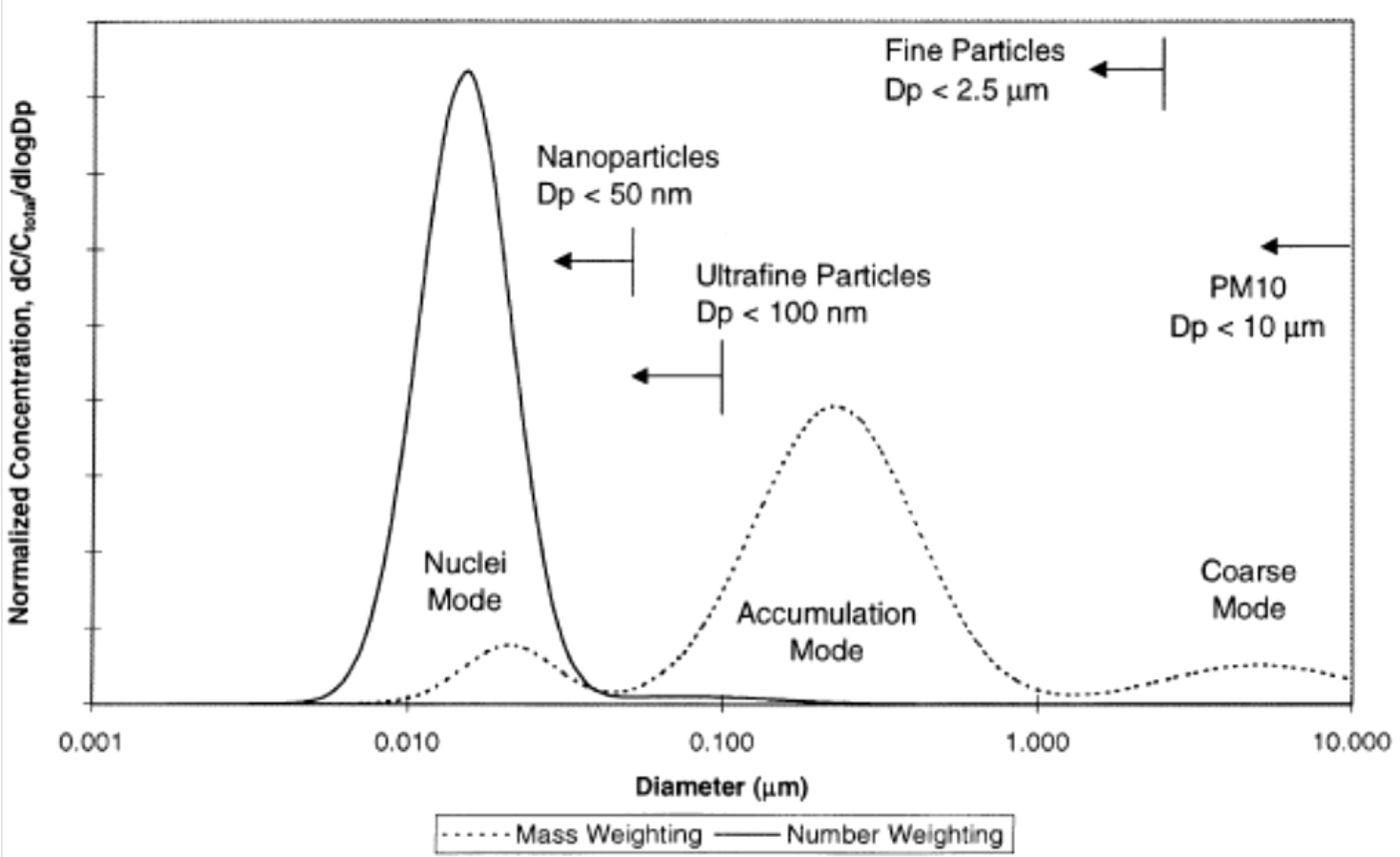

Figure 2-5 Typical particle mass and number weighted distribution (Kittelson, 1998b)

As we can see in the above figure, diesel engine exhaust particle size distribution can be categorized into three modes a) nuclei mode (predominantly volatile particles) b) accumulation mode (a complex mixture of semi-volatile and solid fraction particles) c) coarse mode (particles such as ambient dust). Nuclei and accumulation are two modes of interest with respect to internal combustion engines. The bounding diameters of nuclei mode is not clearly defined. Kittelson in his early works has determined nuclei mode as particle aerodynamic diameters less than $50 \mathrm{~nm}$ (Kittelson, 1998a). However, in a recent work he has redefined this boundary to be a particle less than $30 \mathrm{~nm}$ (Kittelson et al., 2002). This attempt to redefine the boundaries of nucleation mode particles came about after extensive experiments aimed at discerning the volatile fraction of PM through catalytic strippers and heated dilution approaches. Recent works have challenged the existing understanding that all particles below $50 \mathrm{~nm}$ were volatile in nature and should be classified as nucleation mode particles. Also, the proposed European Particle Measurement Programme (PMP) has proposed all solid particles above $23 \mathrm{~nm}$ need to be regulated. The change in viewpoint regarding to nuclei mode particles can be attributed to the advancement in engine and after-treatment technology. Current, heavy-duty diesel and natural gas engines emit particles predominantly in the nuclei and ultrafine region. From Figure 
2-5 it can also be seen from the relative contribution of surface area is several orders of magnitude greater than mass contribution within the nucleation mode size range.

Toxic reactions induced by particulate matter are usually a result of particle surface interaction with tissue or individual cells. Hence, we can easily conclude that greater the surface area of nanoparticles, the greater its effect on cells. The contribution of surface area relative to mass is higher in the nanoparticle size range. Therefore surface area would be a more appropriate metric to correlate nanoparticle emissions and toxicity but all current particulate matter metrics are based on mass contributions. This is due to the fact that regulations for heavy-duty engines are mass based and measurement of surface and number count requires specialized instruments. But surface area analysis and correlation with toxicity responses could lay the foundation for future regulations on the particle size ranges of interest.

\subsubsection{PARTICLE NUMBER}

Particle number count is measured using optical or charge collection methods. Condensation particle counters (CPC) are optical based instruments and electrometers are charge collection based instruments. Particle number count associated with the size distribution as the combination of the two metrics, suggests the count median diameter (CMD) of a log normally distributed particle size distribution. Number based metric is very helpful in determining the nanoparticle concentration in the exhaust. The concurrent measurement of particle size distribution and number concentration is achieved through a combination of a differential mobility analyzer (DMA) and a particle counter. The size segregation through a DMA is achieved through the principles of electrical mobility. The particles are classified using the

electrical mobility diameter of the particles. In general electrical mobility diameter of a spherical particle is closely associated with its Stokes diameter of particles in flow stream (Burtscher, 2001).

Particle number count is a useful metric to discern PM emissions in the size ranges that do not contribute to substantial mass. Particle number count in the nucleation region is highly dependent on exhaust dilution conditions, since number and size of particles is a function of nucleation, condensation and coagulation rates. Unlike PM mass, which is a direct 
representation of the concentration of primary particles emitted from the tailpipe, particle number can be viewed as metric that represents both primary particle concentration and particles formed as result of dilution outside the confines of the exhaust tailpipe (not necessarily representing secondary PM formed in the atmosphere).

The particle number based regulation is a highly debated topic between European legislation and regulatory agencies in the US. This divide is primarily due to the differences in the accepted definition of PM from internal combustion engines. While the USEPA defines particulate matter with dilution and sampling conditions, the European legislation has recently adopted additional solid particle number count metric in the Particle Measurement Programme (PMP) regulations.

One of the key questions faced by US regulatory agencies is the path of which future PM regulation would be adopted. Since, the current regulatory standard of $0.01 \mathrm{gms} / \mathrm{bhp}$-hr, is well over the emission rates of natural gas and DPF equipped vehicles. The path forward in the regulation would be to adopt a number based metric. However, the drive behind a proposed regulation must be to either set a standard that would reduce health effects of nanoparticle emissions, or a standard that would identify failed PM after-treatment systems that would result in higher solid fraction of PM emissions. Since, all regulatory standards are indirectly aimed towards alleviating health issues related to PM emissions, it is necessary to study metrics that better associate toxicity and PM emissions. Particle number count metric would be a suitable path on which to base future regulations; however, there is also a need to link number with other metrics such as surface area, deposition factors and lung deposited surface area (LDSA) to better relate health effects to particle number emissions. The following section will detail the European PMP approach of particle number count measurement.

\subsubsection{EUROPEAN PARTICLE MEASUREMENT PROGRAMME (PMP)}

Particle Measurement Programme (PMP) is an informal group of the United Nations Economic Commission for Europe's (UNECE) Working Party on Pollution and Energy (GRPE). The main objective of this group is to develop a particle measurement technique that would circumvent the short comings of the current mass based technique while addressing health 
concerns from ultrafine particle emissions. Amidst, the divided opinion of the medical community on the dependence of physical composition of PM to its toxicity, the PMP group has adopted a path to detect solid particles in a size range in which accurate number count measurement is feasible, and to further quantify only the contribution of primary tailpipe nanoparticle emissions without considering the effects of dilution. The working group's objectives were aimed at developing a technique that is suitable for both light duty and heavyduty vehicle applications. A report by the Joint Research Commission (JRC) details the development of the PMP methodology and its findings (Andersson et al., 2010). The methodology involves a first stage heated dilution which outputs diluted sample stream at temperatures between $150{ }^{\circ} \mathrm{C}$ and $400{ }^{\circ} \mathrm{C}$, followed by an evaporator section maintained at 300 ${ }^{\circ} \mathrm{C}$ and designed to achieve a residence time of $0.2 \mathrm{sec}$. Finally the particle stream would be cooled, with a secondary dilution system, with HEPA filtered dilution air at ambient temperature. Multiple dilution stages can be used to reduce the particle concentrations below the upper bound of the particle counter. The particle counter should have a $d_{50}$ cut point diameter of $23 \mathrm{~nm}$, in order to exclude volatile particles that may be formed by gas to particle conversion process during secondary dilution (Giechaskiel et al., 2008). On a whole the PMP can be summarized as a methodology which is aimed at counting all particles greater than $23 \mathrm{~nm}$ in diameter and assumed to be solid in physical composition. It is to be noted that, similar to USEPA's definition of TPM, UNECE's definition of solid PM is also based on sampling conditions and not necessarily derived from physical and morphological analysis. The underlying theory behind the UN-ECE PMP is the fact that by heat treating and successive dilution of the exhaust gas, the gas phase compounds present in the sample stream would have achieved low enough partial vapor pressures that would inhibit particle formation through gas phase portioning. As a result the particle stream would consist of mainly particles with solid fractions and volatile material would remain in gas phase.

The current EURO V/VI light duty standard for particle number count has been set at $6 \mathrm{X}$ $10^{11} \# / \mathrm{km}$ (Giechaskiel et al., 2008). A study by Johnson et al evaluated the PN emissions from a MY 1999 international DT466, 7.6 liter engine and a MY 2000 Caterpillar C-15, 15 liter engine in accordance to procedures set forth in the PMP (Johnson et al., 2009). A salient feature of the 
study was the use of a CPC with a low detection diameter of 2-3 nm in addition to the PMP CPC with a modified low detection diameter of $23 \mathrm{~nm}$. The results of the study show that during the chassis dynamometer test cycles that included cruise and UDDS, the particle number counts were well below the UN-ECE light duty regulation for the $23 \mathrm{~nm} \mathrm{CPC}$. However, the lower detection limit CPC detected higher particle number count and in some instances exceeded the $6 \times 10^{11} \mathrm{\#} / \mathrm{km}$ limit. Also, on-road testing of a vehicle equipped with the C-15 diesel engine resulted in $23 \mathrm{~nm} \mathrm{CPC}$ number count well below the UN-ECE limit. However, higher particle counts were observed to exceed the limit during certain high load operations. One of the important findings of the study has been the detection of "solid particle" $<23 \mathrm{~nm}$ in size (Johnson et al., 2009). Similar observations have been referenced in unpublished works by Herner et al. and Ayala et al. (Johnson et al., 2009). The results of the study can be interpreted as the possibility of particle formation mechanisms that manifests themselveself as "solid particle" as per PMP methodology, while in actuality could be sulfuric acid based particles or renucleating light hydrocarbons that form particles below 2-3 $\mathrm{nm}$. However, this observation also offers the question of the total fraction of particles over $23 \mathrm{~nm}$ that are associated with condensation of light hydrocarbons and sulfuric acid droplets.

From the PMP perspective particle number count metric can be viewed as a highly sensitive metric that can measure PM emissions at low mass emission rates. Particle number count as a work specific metric or a distance specific metric can be used effectively to monitor after-treatment system performance and compliance. However, in order to address potential health effects of nanoparticle emissions from heavy-duty vehicles alternate metrics must be considered that better link toxicity and PM composition.

\subsubsection{PARTICLE MASS}

Regulatory standards both in Europe and in the United States have adopted a mass based system as the primary metric in which PM would be regulated. Particulate matter mass measurements are performed by a gravimetric method in which a suitable filter media (Teflon based) is used to collect PM samples at standardized sampling conditions and subsequently weighed over a sensitive microbalance to discern the mass of emissions. 40 CFR part 1065 
prescribes various sampling conditions that must be achieved before sample collection over the filter. Table 1 lists the sampling conditions as prescribed in 40 CFR Part 1065.

Table 140 CFR Part 1065 PM sampling requirements (USEPA, 2012)

\begin{tabular}{|c|c|}
\hline Dilution Air Quality and Temperature & HEPA filtered, $25 \pm 5{ }^{\circ} \mathrm{C}$ \\
\hline Dilution Ratio & Primary: Minimum 2:1; Overall: Minimum $5: 1$ \\
& to $7: 1$ \\
\hline Residence Time & Minimum of $0.5 \mathrm{~s}$ \\
\hline Sample Temperature & $47 \pm 5{ }^{\circ} \mathrm{C}$ \\
\hline Filter Face Velocity & Approx. $100 \mathrm{~cm} / \mathrm{s}$ \\
\hline
\end{tabular}

These prescribed sampling conditions are designed to minimize sampling artifacts and provide a repeatable and consistent PM collection process and not necessarily represent realworld PM formation mechanisms. The sampling procedures prescribed in 40 CFR Part 1065 (USEPA, 2012) are a result of an extensive study conducted by Southwest Research Institute (SWRI) as part of the E-66 study (Khalek, 2007). The study investigated various parameters that affect sample loading on the filter as applicable to exhaust from DPF equipped and ultra low PM emission engines such as natural gas (Khalek, 2007). USEPA regulations adopted the findings of this study to prescribe a sampling methodology that would be associated with the least sampling artifacts, and be able to capture the volatile fraction of PM more efficiently on a filter material.

Mass based metric faces serious limitations at ultra low PM emission rates. In the case of DPF equipped diesel engines, the mass emissions are well below the detection limits of the gravimetric system and hence high variations in measured mass is not vital to the compliance of the engine to regulations. Similarly, in natural gas engines the soot free combustion produces particles predominantly in the nucleation region, which does not impart any mass to the collected PM. 
However, PM toxicity studies commonly reference toxicity response to mass dose emitted. In case of ultra low level PM emissions rate, inaccuracies in mass measurements would impart an error in the mass normalized PM toxicity result. To alleviate this setback, most studies adopt extended sampling times and high volume flow sampling to increase mass loading on the filter, in order to observe a better toxicity response and sufficient mass to measure accurately. The approach of both extended sampling times and high volume flow has been widely debated due to the positive and negative artifacts that may be imparted to the PM sample as result of extended sample flow across the filter or high filter face velocities that could possibly strip volatile material from collected PM. Since, no study has accurately characterized the effect of sampling artifacts on toxicity response, high volume sampling and extended sampling times are continued to be widely used sampling procedures for increasing filter sample loading.

The inaccuracies in physical measurement of PM mass can be addressed through an approach of PM mass calculation using particle number count and size distribution classification. Many studies have developed particle effective density functions that have related particle diameter to their mass. Hence, by measuring particle number count and size distribution, it is possible to calculate mass of PM (Liu et al., 2012). A study by Liu et al. focused on the strategies that would help detect ultra-low PM emissions at better resolution and with greater repeatability. The study investigated two alternate methods a) chemical reconstruction of mass though separate chemical speciation of exhaust and b) an integrated particle size distribution method (IPSD). The study found that the IPSD method provided a methodology to quantify PM mass at ultrafine and nanoparticle size ranges with high levels of resolution and repeatability (Liu et al., 2012).

The study investigated various effective density functions from important literatures and found that the function developed by Maricq et al. matched closely with gravimetric PM results of a non-DPF equipped engine. Figure 2-6 shows the density plots developed by Maricq in 2000 and 2004 and Park et al. in 2003. It can be seen that the Maricq et al. (Maricq and Xu, 2004) density function developed in 2004, shows a constant unit density for all particles within the nucleation mode and part of the accumulation mode (Liu et al., 2012). Both natural gas engines and DPF equipped diesel would most often emit PM within these size ranges. 


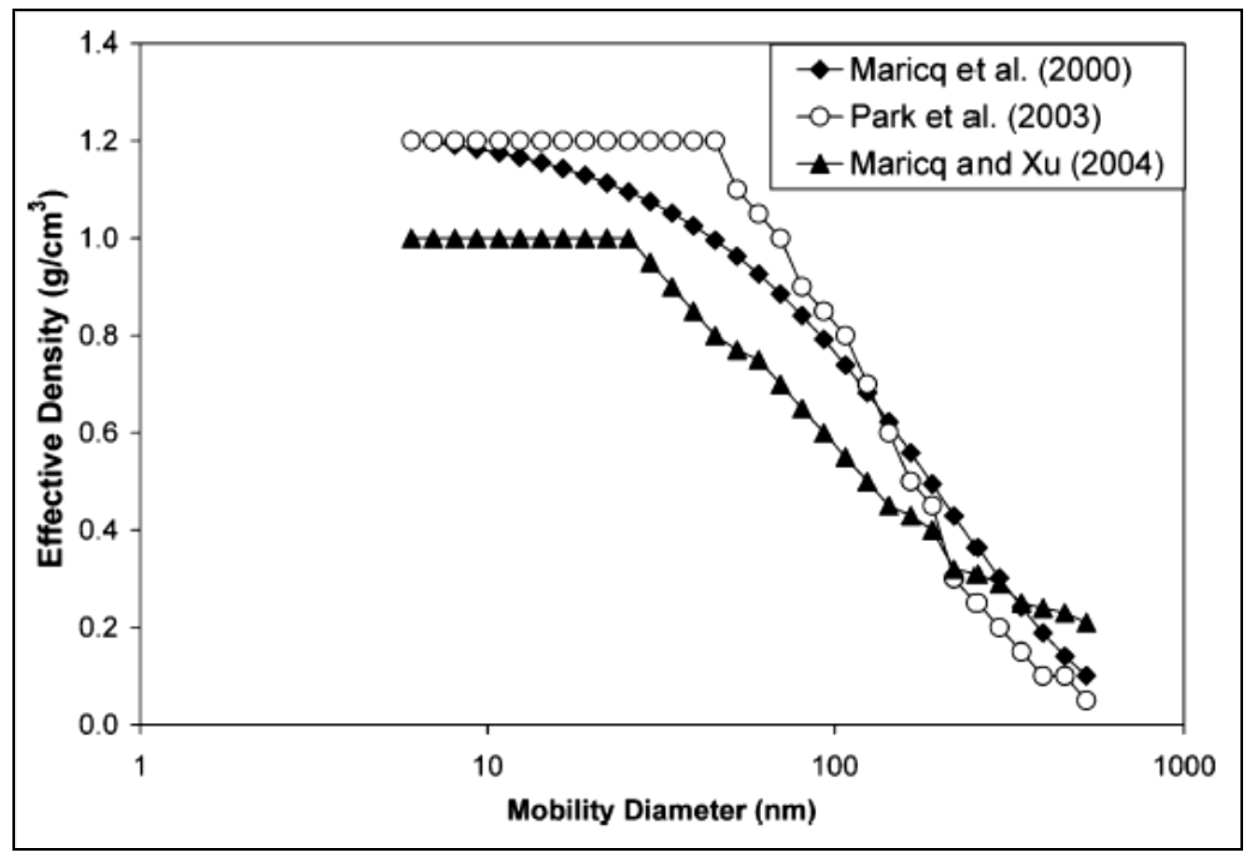

Figure 2-6 Effective density functions developed by various researchers (Liu et al., 2012)

Maricq et al.'s density distribution function is given in Equation 1 (Maricq and Xu, 2004).

$$
\rho_{\text {eff }}=1.2378 * e^{-0.0048 * D p}
$$

Equation 1

With the effective density function relating particle diameter $\mathrm{Dp}$ and particle density over the entire size distribution, mass calculation can be performed by assuming all particles to be spherical, with $\mathrm{N}_{\mathrm{i}}$ number of particles in each size bin. Hence the mass calculation equation can be expressed as (Liu et al., 2012).

$$
\text { PMmass }=\sum_{i=\text { lower D }}^{\text {Higher } D p} \rho_{e f f, i} *\left(\frac{4}{3}\right) * \pi *\left(\frac{D p_{i}}{2}\right)^{3} * N_{i}
$$

\section{Equation 2}

The approach using particle number count to calculate mass could prove more accurate in relating toxicity response to total mass of PM. Instruments such as TSI's Engine Exhaust Particle Spectrometer (EEPS) could be versatile in discerning mass for transient engine operations and to further calculate mass for ultra-low PM emissions vehicles. 


\subsubsection{PARTICLE SURFACE AREA}

Nanoparticle surface area has long been considered to be a metric that better represents health effects of nanoparticles. From Figure 2-5 we see that particles of smaller diameters offer a greater surface area for interaction than particles of larger diameter. When we consider the case of inhalation of ultrafine and nanoparticle size range engine exhaust particles, we are forced to consider the possible interactions these particles will undergo in the airways and in gas exchange regions of the lungs. Hence, a more appropriate metric would be the surface area as it represents the total amount of particle that would physically react with cells and tissue. Both number and mass metrics consider particles as a bulk system and toxicity reactions as function of the entire particle composition. However, internal combustion engine particle composition is complex and could consist of multiple layers of organic and inorganic fractions. Hence it is important to know what fraction of the particles offer the greatest surface for interaction. Consequently particle surface area can be viewed as a metric that indirectly takes into account the particle morphology and the surface composition of the particles.

The importance of particle surface area is more pronounced for particles that are insoluble in lung fluids or water. In the case of particles that are soluble in water or lung fluid, a bulk metric such as number and mass would be sufficient in correlating toxicity with PM emissions. However, if in case of an insoluble particle, the composition of the outer surface of the particle and the amount of surface area offered would be the critical metric that would provide an appropriate correlation to toxicity response. One important metric that is analogous to particle surface area is the lung deposited surface area (LDSA), which takes into account the deposition fraction of particles entering into the human airway. LDSA could be a useful metric in developing better correlations between PM composition and toxicity response.

Very few studies have attempted to correlate particle surface area and health effects. Studies by Sager and Castranova ((Sager and Castranova, 2009) and Oberdorster et al. (Oberdorster et al., 1994) are some of the important works that have suggested that surface area dosing of particles correlates better with inflammatory responses in tissues of the human airways. A review of literature involving respiratory health effects of diesel particulate matter (Ristovski et al., 2012) states that three physio-chemical properties of diesel particulate matter 
namely :a) surface area, b) presence of transition metals in PM and c) adsorbed organics on surface of DPM are to be considered while linking PM emissions and health effects (Ristovski et al., 2012).

Discerning the particle composition on the surface of a nanoparticle is a challenging task, and as a result surface area metric has been a highly debatable metric in the health community. Short comings of the surface area metric have forced particle mass as the de facto metric for associating health effects. However, LDSA and the representativeness of diffusion charger instrument's response as an indicator of LDSA could change future perspective of the surface area metric (Wilson et al., 2007). The following section will review in detail the LDSA metric and its associated findings.

\subsubsection{LUNG DEPOSITED SURFACE AREA (LDSA)}

LDSA is a very effective metric in refining the surface area metric as it pertains to human inhalation. From a health perspective the total surface area of a particle size distribution would not represent the actual surface area of particles that will interact with airway tissues and cells. This is due to the fact that as particles travel through the airways, inertial and diffusion forces act upon particles, which will result in deposition in various regions of the airways. Hence, a deposition curve is required to relate the total surface area of particle to the deposited surface area of the particle. Hence, LDSA represents a surface area that takes into account the particle deposition factor for a human lung.

Traditionally LDSA could be calculated only by measuring the particle size distribution and number concentration to calculate the total surface area of spherically assumed particles followed by using deposition fractions to calculate LDSA. However, recent studies have shown that by coincidence, diffusion charging based instruments have been observed to produce a response that is very similar to LDSA. Wilson et al in their work with an electrical aerosol detector observed that the instrument response was well correlated with LDSA calculations (Wilson et al., 2007). The study used a TSI electrical aerosol detector (EAD) and scanning mobility particle sizer (SMPS) to measure particle size distribution and particle length. One of the important findings of the study was that it observed a strong correlation between the signal 
from the EAD and the calculated deposited surface area (particle number concentration multiplied by the deposition fraction for a certain diameter). The study further concluded that even with changes in particle size distribution, the signal from EAD correlated well with deposited surface area (Wilson et al., 2007). Hence, the EAD by itself could be a versatile instrument in directly suggesting the LDSA metric of a particle stream.

\subsubsection{DEPOSITION FRACTION}

Deposition fraction (DF) is an important parameter in discerning health effects of nanoparticles, and also to optimize respiratory drug delivery methods. DF defines the percent deposition of particle of various sizes during its transport within the human airway system, while being subjected to various forces such as gravitational settling, inertial settling and Brownian and diffusion movements. The respiratory tract consists of three regions namely: a) nose and head airways b) tracheobronchial region c) alveolar region. Deposition models have been a focus of research from early 1930s'. Findeisen developed the first lung deposition model in 1935. Since then the Findeisen model has undergone modifications in 1950 by Landahl and Beeckman in 1965 (Ensor, 2011). However, from the early 1960s the model developed by the International Commission on Radiological Protection (ICRP) has been the widely accepted model used for drug delivery and associating nanoparticle inhalation with health risks. The toal fraction of particles deposited in human lungs cab be estimated using Equation 3 (Ensor, 2011).

$$
D F=0.0587+\frac{0.911}{1+\exp (4.77+1.485 \ln D p)}+\frac{0.943}{1+\exp (0.508-2.85 \ln D p)}
$$

Equation 3

The above equation represents the deposition model for both men and women average for three types of physical activity that includes resting, light exercise and heavy exercise. The above equation was obtained through a curve fit of experimental data fitted to produce a deposition curve as a function of particle diameter $\mathrm{Dp}$. 


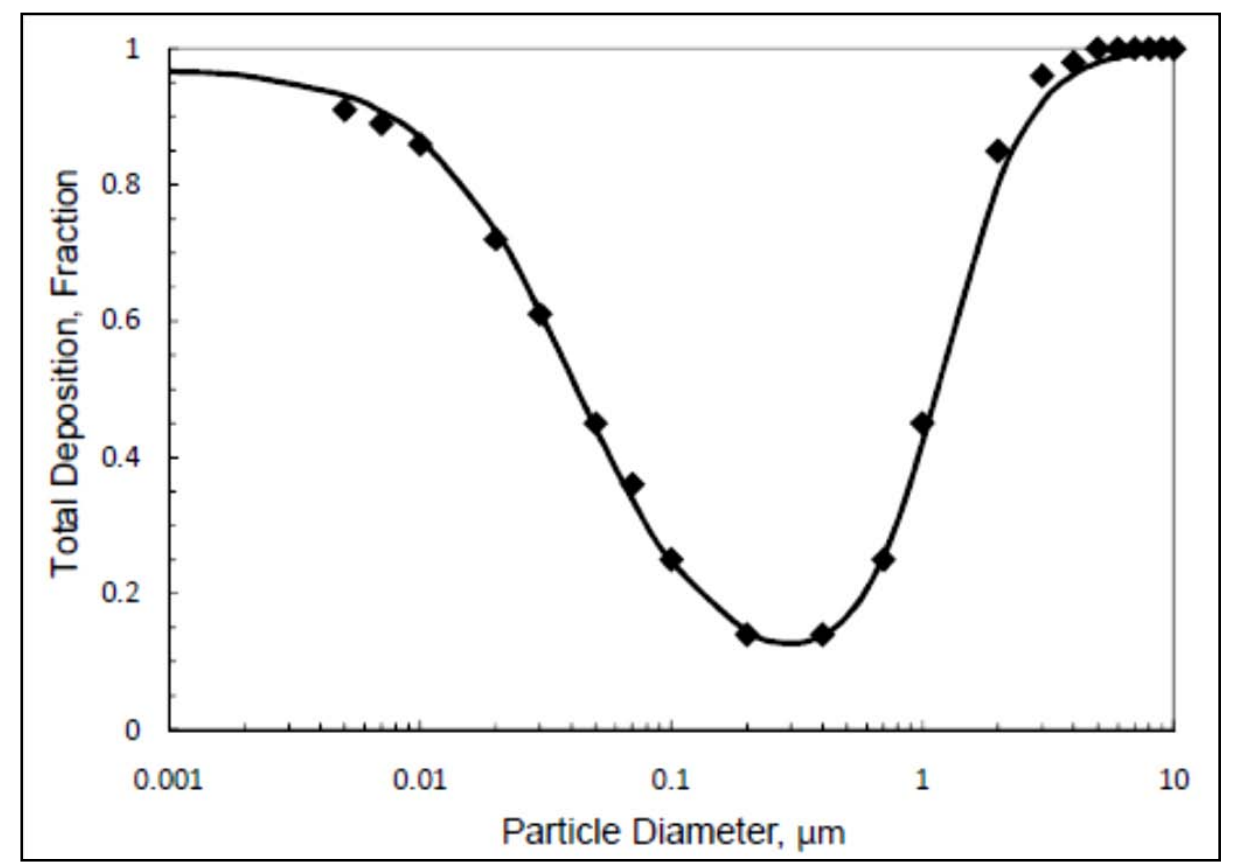

Figure 2-7 Measured and theoretical curve for particle DF as adapted from (Ensor, 2011)

The type of particle diameter used in the model vary with the region in which the model is applicable. Gravitational and inertial settling and thermodynamic processes such as diffusion dominate particle deposition. Hence, in regional where gravitational settling and inertial settling are dominant, the aerodynamic diameter is used and in regions where diffusion is dominant, thermodynamic diameters are used. However, most particle instruments measure the electrical mobility diameter of the particle, which is closely approximated to stokes diameter for a spherical particle. Hence, while attempting to discern particle DF from mobility based instruments, diameter conversion is to be taken into account. Wilson et al. in their study suggest that electrical mobility agrees well with particle diameters associated with diffusion based measurements (Wilson et al., 2007). Hence, if Dp in the above equation is assumed to be stokes diameter, then the electrical mobility diameter measured directly from SMPS or EEPS can be used to represent DF as a function of particle diameter.

The subject of DF can be further extended to discern regional DF in the three regions mentioned earlier. The following equations represented in Equation 4, Equation 5 and Equation 6 signify the DF values for the different regions in the airway ( $\mathrm{Wu}$ and Allen). The equations 
represented here are derived from the ICRP model as applicable to the different regions of the lungs.

a) Head Airways

$$
D F_{\text {head }}=I F\left(\frac{1}{1+\exp (6.84+1.183 \ln D p)}+\frac{1}{1+\exp (0.924-1.885 \ln D p)}\right)
$$

Where

$$
I F=1-0.5 *\left(\frac{1}{1+0.00076 D p^{2.8}}\right)
$$

\section{Equation 4}

b) Tracheobronchial region

$$
D F_{T B}=\left(\frac{0.00352}{D p}\right) *\left(e^{\left(-0.234(\ln D p+3.40)^{2}\right)}+63.9 * e^{\left(-0.819(\ln D p-1.61)^{2}\right)}\right)
$$

Equation 5

c) Alveolar region

$$
D F_{A L}=\left(\frac{0.0155}{D p}\right) *\left(e^{\left(-0.416(\ln D p+2.84)^{2}\right)}+19.11 * e^{\left(-0.482(\ln D p-1.362)^{2}\right)}\right)
$$

Equation 6

Figure 2-8 shows the percentage DF calculated from the ICRP model for two different breathing types as a function of particle diameter. The curves show that particles below the ultra fine size range $(<100 \mathrm{~nm})$ have a significant deposition fraction in the alveolar region. Hence particles with smaller diameters can be viewed as a more potent threat to human health as they enter into the oxygen exchange regions of the lung. Particles in the accumulation region are observed to have a greater deposition in the tracheobronchial region, and as result might contribute to significant inflammatory responses in the cells of the respiratory path. Although the tracheobronchial region acts to filter most inhaled particles, greater surface area contribution combined with potent particle surface coating such as organics and transition metals can induce ROS responses in the cells and contribute to breakdown of the defense system. 


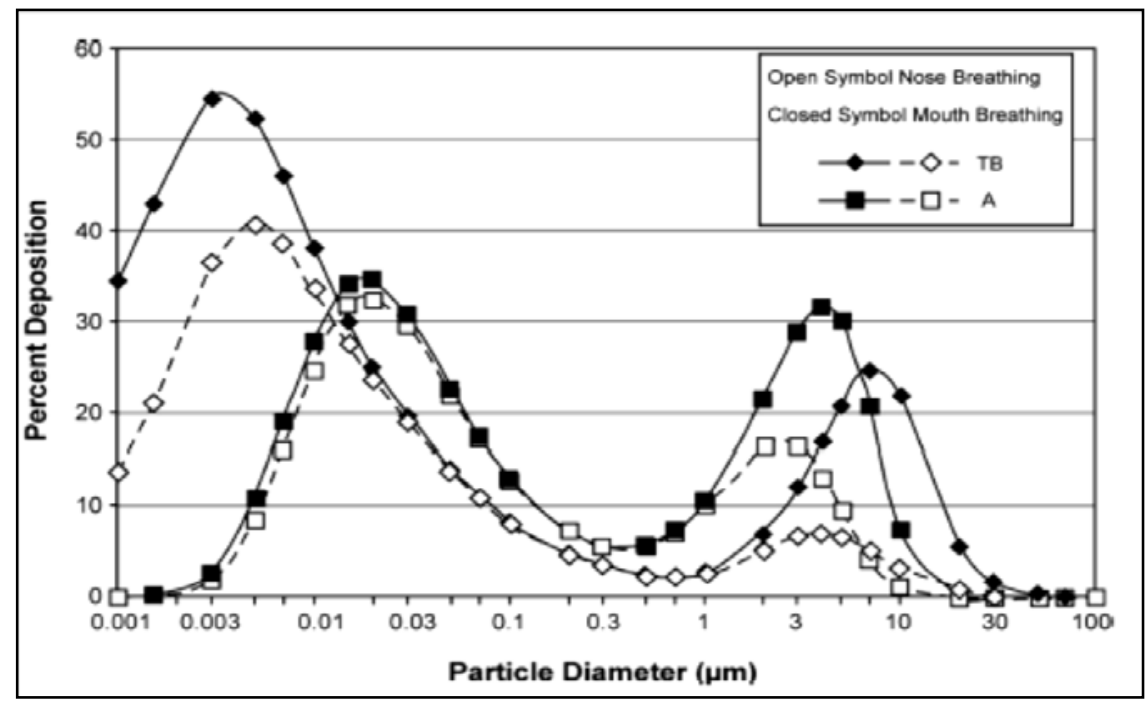

Figure 2-8 Percentage DF curve as function of particle diameter adapted from (Wilson et al., 2007)

This study will present an analysis of the deposition fraction of nanoparticle emissions from heavy-duty natural gas engines and further correlate the toxicity responses to LDSA as calculated using particle size distribution and DF, in addition to measurements obtained from a TSI EAD.

\subsection{CLOSING STATEMENTS}

Literatures cited in this work have focused on detailing research that has focused on investigating and characterizing particulate matter composition and its associated health effects. It can be concluded that there is sufficient lack of information in the literary community with respect to toxicity responses based on compositional characteristics of PM. Although studies have shown the fractional contribution of various elements of PM such as metals and organics to specific toxicity responses, the health effects of PM as a whole pertaining to different operational characteristics of the engine are largely unknown. This void in knowledge can be widely attributed to the lack of connection between engine studies that represent realworld conditions to health effects research that does not consider the complexities of engine design, operation and durability. PM emissions are a function of many factors and the corresponding health effects should also be closely related to it. However, almost no toxicological study approaches PM emissions and health effects from an engine and its associated technology stand point. 
Health effects of PM emissions cannot be represented by a single unified metric. Hence, different assay are used to study toxicity responses from various fractions of PM. This study is unique in its approach by simultaneously investigating toxicity response from three different assays. The results of the study will provide statistical correlation to size resolved chemical composition of PM with its toxicity responses.

Literatures cited in this work have highlighted the drawbacks of using the mass based metric in determining toxicity responses of PM emissions from advanced heavy-duty engines. Particle LDSA, number and surface area are some of the metrics that have proven to be suitable for normalizing PM toxicity. Combination of TSI EAD and TSI EPPS used in this study has helped provide an analysis of the particle surface area as calculated from a simple number count measurement with the TSI EEPS and through a dedicated LDSA response instruments the EAD. Hence, the result of this study provides a link between observed instrument response from an EAD and an EEPS to a probable toxicity response.

The study has adopted a novel approach to highlight engine operation, technology and durability as a main factor in relating particle emissions and its subsequent toxicity. The approach attempts to provide a complete analysis of source to end effect relationship in PM emissions. 


\section{CHAPTER 3 - EXPERIMENTAL SETUP}

\subsection{VEHICLE EMISSIONS TESTING LABORATORY}

The West Virginia University Transportable Heavy Duty Vehicle Emissions Laboratory consists of transportable heavy-duty chassis dynamometer and a transportable emissions measurement container.

\subsubsection{CHASSIS DYNAMOMETER}

The chassis dynamometer test bed consists of rollers, flywheel assembly, eddy current power absorbers, differentials, hub adapter, torque and speed transducer built onto a tandem axle semi trailer (see Figure 3-1 and Figure 3-2). The hydraulic jack on the chassis dynamometer test bed is functional in setting the test bed on the ground and onto the trailer. The various components of the chassis dynamometer are discussed in detail below.

- Rollers: The chassis dynamometer consists of a set of two paired rollers in the front which supports the single or forward drive axle and a set of single roller at the back in order to support the rear axle of tandem axle vehicles. The rear pair of rollers can be placed in three different positions to accommodate tandem spacing of 4 to $5 \mathrm{ft}$ $(1.22-1.52 \mathrm{~m})$ and each roller is $12.6 \mathrm{inch}(32 \mathrm{~cm})$ in diameter with their axis along the length of the test bed. Each pair of rollers is linked by a flexible coupling to have uniform rotational speed on either side of the vehicle and the coupling was designed to accept $20 \%$ of the wheel torque in case of any imbalance due to uneven surface at the test location.

- Hub Adapters: The hub adapters are used to couple the engine drive axle with the flywheel assembly and eddy current power absorber via torque and speed transducer. The adapter is made of a 0.5 inch $(13 \mathrm{~mm})$ thick aluminum plate of diameter $1.8 \mathrm{ft}(0.55 \mathrm{~m})$.

- Load Simulation System: The load simulation system consists of a flywheel assembly, an eddy current power absorber, a speed and torque transducer, double differentials and universal couplings on either side of the vehicle to be tested as shown in the figure below. The power from the vehicle's drive axle is transmitted to 
the flywheel assembly and power absorbers by a hub adapter which is connected to a 24 inch $(61 \mathrm{~mm})$ long spline shaft running into a pillow block. The spline shaft is connected to the speed and torque transducer by a universal coupling which can withstand torque up to $16,415 \mathrm{lb}$-ft $(222,256 \mathrm{~N}$-m) on either side. The speed and torque transducer is capable of providing the data logging computer with time varying output torque at a rate of $10 \mathrm{~Hz}$. The torque transducer drives a second shaft via companion flange. This shaft transfers power to a right-angle speed increasing drive, a double reduction differential with a ratio of 1:3.65 which drives the flywheel assembly and a second differential. The second differential with a ratio of 1:5.73 drives the eddy current power absorbers.

- Flywheel Assembly: The flywheel assembly is designed to simulate vehicle gross weights of 40,000 to $66,000 \mathrm{lb}$. With the maximum being $40,000 \mathrm{lb}(18,144 \mathrm{~kg})$ at a wheel diameter of $4 \mathrm{ft}(1.22 \mathrm{~m})$ and $66,000 \mathrm{lb}(30,000 \mathrm{~kg})$ at a wheel diameter of $3.25 \mathrm{ft}(1 \mathrm{~m})$. The flywheel assembly consists of a drive shaft with four drive rotors running in two pillow blocks. Each drive shaft supports eight flywheels of different sizes with bearings resting on the shaft. By selectively engaging the flywheels to the drive rotors, vehicle mass can be simulated in $250 \mathrm{lb}(113 \mathrm{~kg})$ increments.

- Eddy Current Power Absorbers: A Mustang model CC300 air cooled eddy current dynamometer mounted on two bearings is used as power absorbers. The power absorbers are used to simulate load due to rolling friction of the tires and the aerodynamic drag resistance. The eddy current dynamometer has the capability of absorbing $300 \mathrm{hp} \mathrm{(224} \mathrm{kW)} \mathrm{continuously} \mathrm{and} 1000$ hp (745.7 kW) intermittently during peak operation. Dynamometer load at any speed is controlled by the direct current supplied to the coils and the power absorbed is measured by the torque arm force transducer (load cell). 


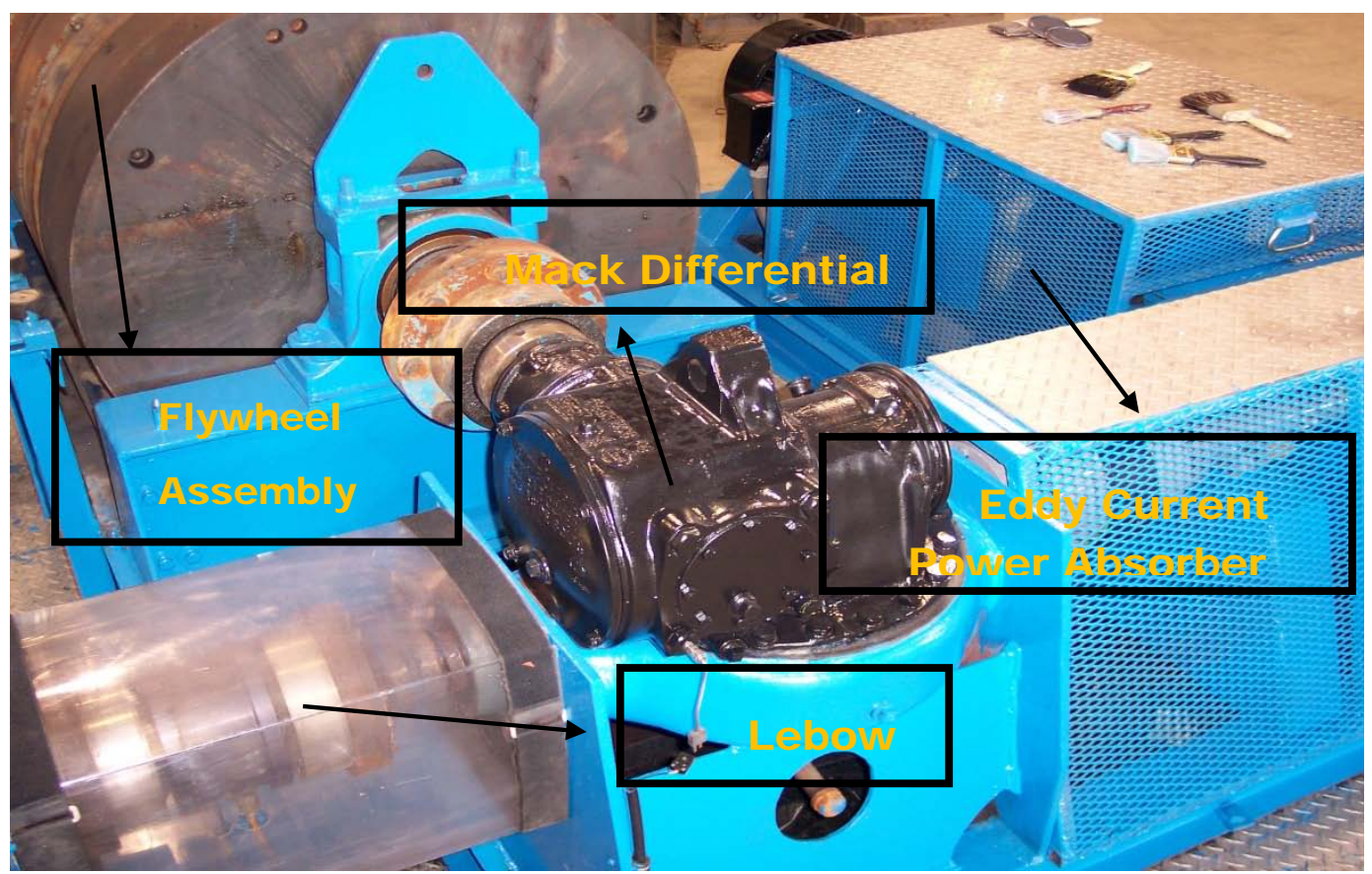

Figure 3-1 Components of a Chassis Dynamometer

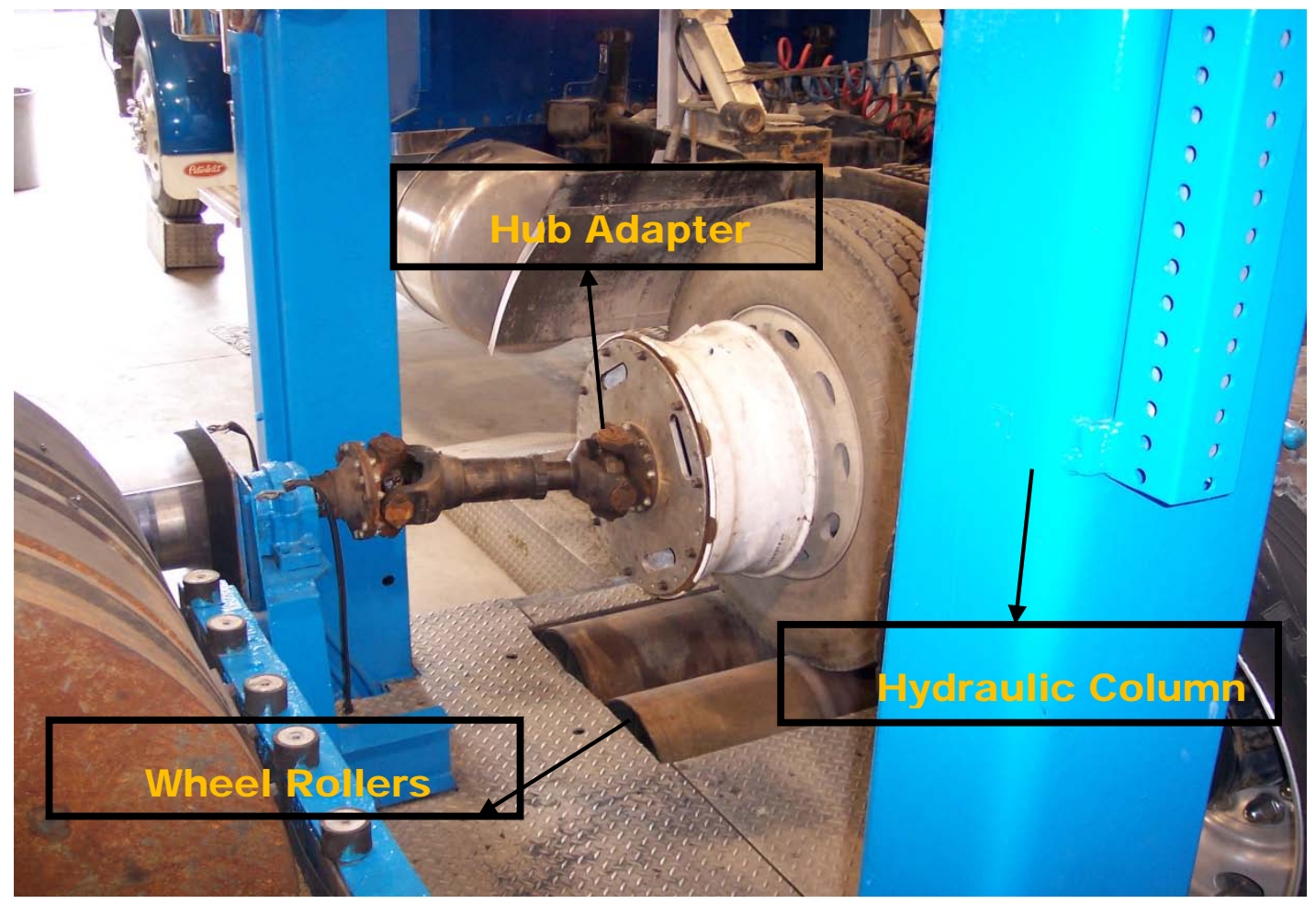

Figure 3-2 Connecting and supporting structure of chassis dynamometer

While the driver is responsible for the control of the speed, the transient torque must be controlled by an automated system. The load supplied by the flywheels simulates the static 
weight on the engine and is controlled by their rotational speed, while the load due to rolling friction and wind drag is simulated by the eddy current dynamometer. The eddy current dynamometer is controlled by a Dyn-Loc IV control system provided by Dyne-Systems. The DynLoc IV control system operated by a PID control loop where "P" stands for proportional control in which the controller calculates error between the actual and the desired output resulting in a restoration signal linearly proportional to the error. "I" stands for integral control in which the controller calculates the average error over a time and provides a restoring signal which is the product of the error and the time the error persisted. It is used to restore the original set point. D stands for differential control in which the controller calculates the rate at which the set point is changed and produces a corrective signal to reach the set point quickly. Hence, PID controller provides a fast and smooth response in controlling the transient set points. During the test, the power absorbers receive the torque set point from the dyne-loc controller. The set point is equal to the road load power and it is calculated using the following Equation 7 :

$$
P_{r}=\left(C_{r} * M * g+\left(\frac{1}{2}\right) * \rho_{a} * C_{d} * A * V^{2}\right) * V
$$

Equation 7

Where:

$$
\begin{aligned}
& P_{r}=\text { Road load power } \\
& C_{r}=\text { Coefficient of rolling resistance } \\
& M=\text { Vehicle gravitational mass } \\
& \rho_{a}=\text { Air density } \\
& A=\text { Frontal area of vehicle } \\
& C_{D}=\text { Coefficient of drag } \\
& V=\text { Vehicle speed }
\end{aligned}
$$

The updating of set points and data acquisition are performed at $10 \mathrm{~Hz}$. 


\subsubsection{TRANSPORTABLE EMISSIONS MEASUREMENT SYSTEM (TEMS)}

The housing for the new Transportable Emissions Measurement System (TEMS) is a reconstructed $9.1 \mathrm{~m}$ (thirty-foot) long cargo container which houses a high efficiency particulate filter (HEPA) primary dilution unit, two primary full-flow dilution tunnels, a subsonic venturi, a secondary particulate matter sampling system, a gaseous emissions analytical bench instrumentation system, a computer-based DAQ and control system, a heating, ventilating and air conditioning (HVAC) system, and chassis dynamometer control systems. Figure 3-3 shows the schematic of the transportable laboratory container. The two primary dilution tunnels inside the container, of $0.46 \mathrm{~m}$ (18 inches) ID and $6.1 \mathrm{~m}$ (20 feet) long, were designed to provide dedicated measurement capability for both low PM emissions ("clean") vehicles (with the upper tunnel referred as the "clean tunnel"), as well as traditional diesel-fueled vehicles with high PM levels (lower tunnel referred as "dirty tunnel"). This provision reduces tunnel history effects between test programs of differing exhaust emission composition. A stainless steel plenum box houses two HEPA filters for filtering primary dilution air, as well as twin dual-wall exhaust transfer inlet tubes dedicated as exhaust inlets for the upper and lower tunnels. The HEPA plenum is connected into the main dilution tunnels, which are selectively connected to the subsonic venturi via stainless elbow sections. The air compressor and two vacuum pumps are installed inside a noise isolating overhead. An air tank stores compressed air and provides shop air to the zero air generator (a device removes PM and THC) for instrumentation use. A PM sampling box for the secondary dilution tunnels is located alongside the primary tunnels, downstream of tunnels' sample zones. The secondary PM dilution tunnel of either the dirty or clean tunnel is connected to the PM sampling box for PM measurement during the test. Figure 3-4 shows the TEMS container on the transportation Landoll 435 trailer. 


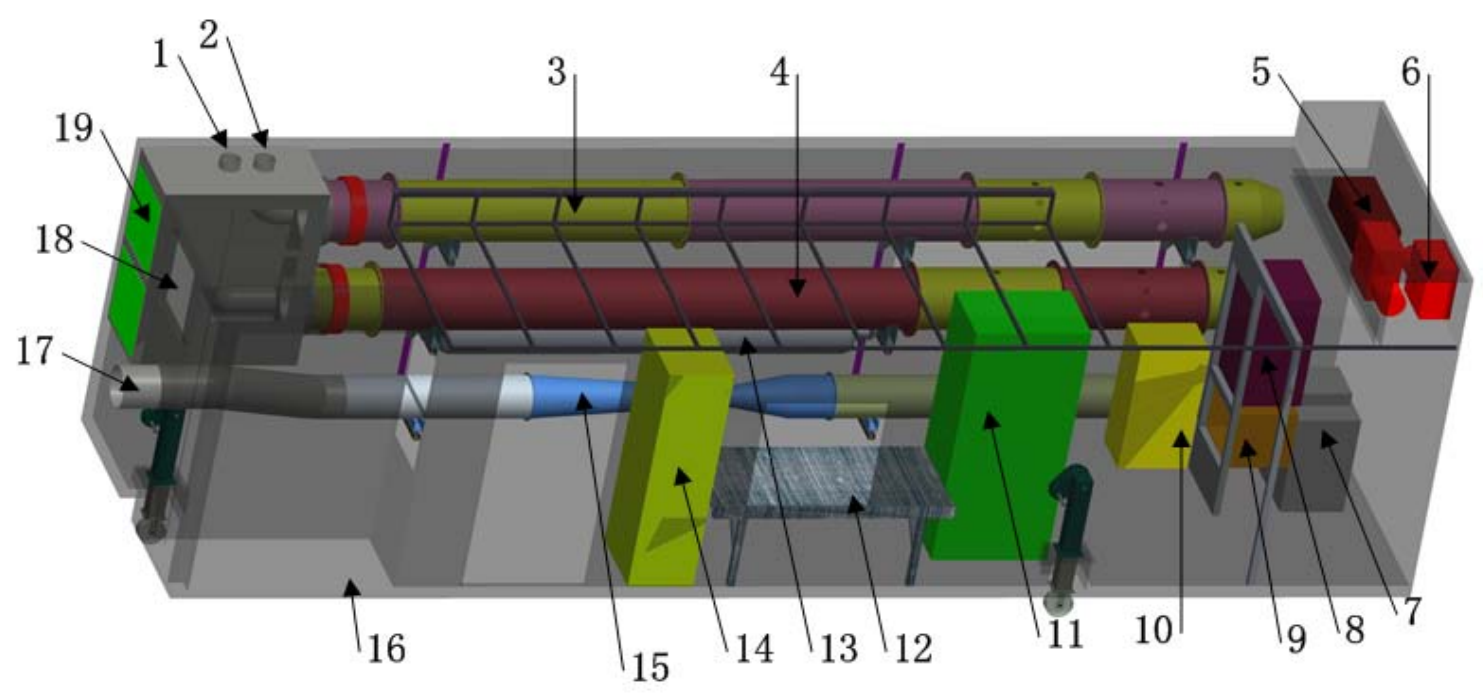

Figure 3-3 Schematic of the TEMS container

1- Exhaust inlet of dirty tunnel; 2- Exhaust inlet of clean tunnel; 3- Clean tunnel; 4- Dirty tunnel; 5Air compressor; 6- Vacuum pumps; 7- Oven; 8- PM sampling box; 9- Glove box; 10-Zero air generator; 11- MEXA-7200D motor exhaust gas analyzer; 12- Computer table; 13- Air tank; 14- DAQ rack; 15SUbsonic venturi; 16- Air conditioner deck; 17- Outlet to blower; 18-Ventilation fan; 19- HEPA filters

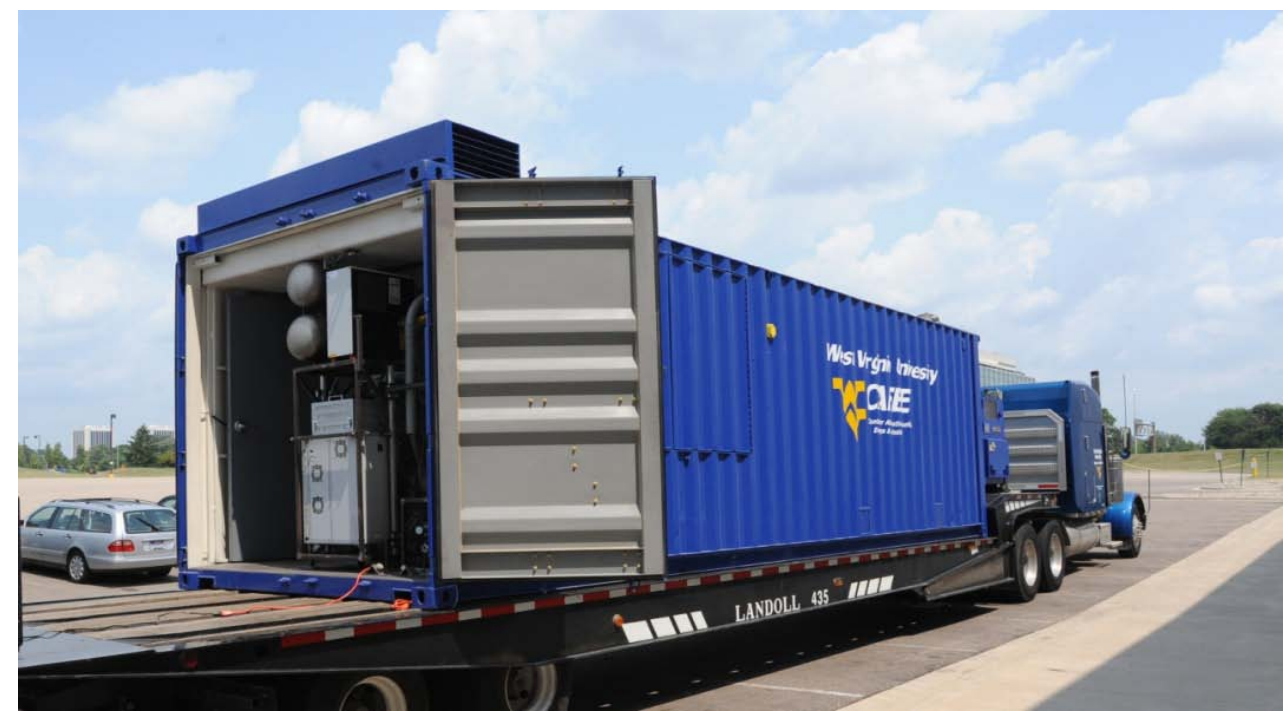

Figure 3-4 View of the TEMS container at the test facility 


\subsubsection{GASEUOUS EMISSIONS SAMPLING SYSTEM}

The gaseous emissions measurement system was designed to be capable of measuring raw exhaust and diluted emission levels. Emissions levels vary continuously over transient test cycles. Final emissions values must be determined with correction for background level. The background sample flow is taken from a sample probe located immediately after the HEPA filters inside the plenum box. A diluted sample flow is drawn from the sample probe installed at the primary sample zone, providing a bag of composite diluted exhaust, which is analyzed along with the background bag. While the purpose of the background batch sampling is to correct for background gaseous levels, the diluted batch sampling provides for a check on integrated continuous values for quality control purposes. In some cases, where the emissions vary over a wide concentration range over a cycle, a dilute bag analysis may also provide a more accurate assessment of those species than can be obtained by integration. This is often the case for CO from legacy diesel vehicles over severe transient cycles.

The container is equipped with Horiba MEXA 7200D motor exhaust gas analyzer for gaseous measurements from the dilution tunnel. The MEXA7200D is capable of measuring all regulated emission species that include $\mathrm{THC}, \mathrm{CO}, \mathrm{CO}_{2}, \mathrm{NO}_{\mathrm{x}}$ and methane through a nonmethane cutter equipped secondary hydrocarbon channel. The unit can be fitted with various analyzer modules, and the current configuration consists of AIA-721A CO analyzer, an AIA-722 $\mathrm{CO} / \mathrm{CO}_{2}$ analyzer and a CLA-720 "cold" $\mathrm{NO}_{x}$ analyzer part of the cold sample stream and the FIA-725A THC analyzer and CLA-720MA NO analyzer part of the heated sample stream.

\subsubsection{PM SAMPLING AND MEASUREMENT SYSTEM}

The measurement container houses the PM sampling system for the transportable laboratory. However, the measurement system of pre-weighing and post weighing the gravimetric filters are carried out in Morgantown, WV at the WVU facility which houses a class 1000 clean room, with controlled environment for accurate weighing of the filters. The measurement system is operated with in-house developed software to calibrate the scales, perform measurements, and also to monitor the filters history. The schematic of the on-board PM sampling system is as shown in Figure 3-5

49 | $\mathrm{P}$ a g e 


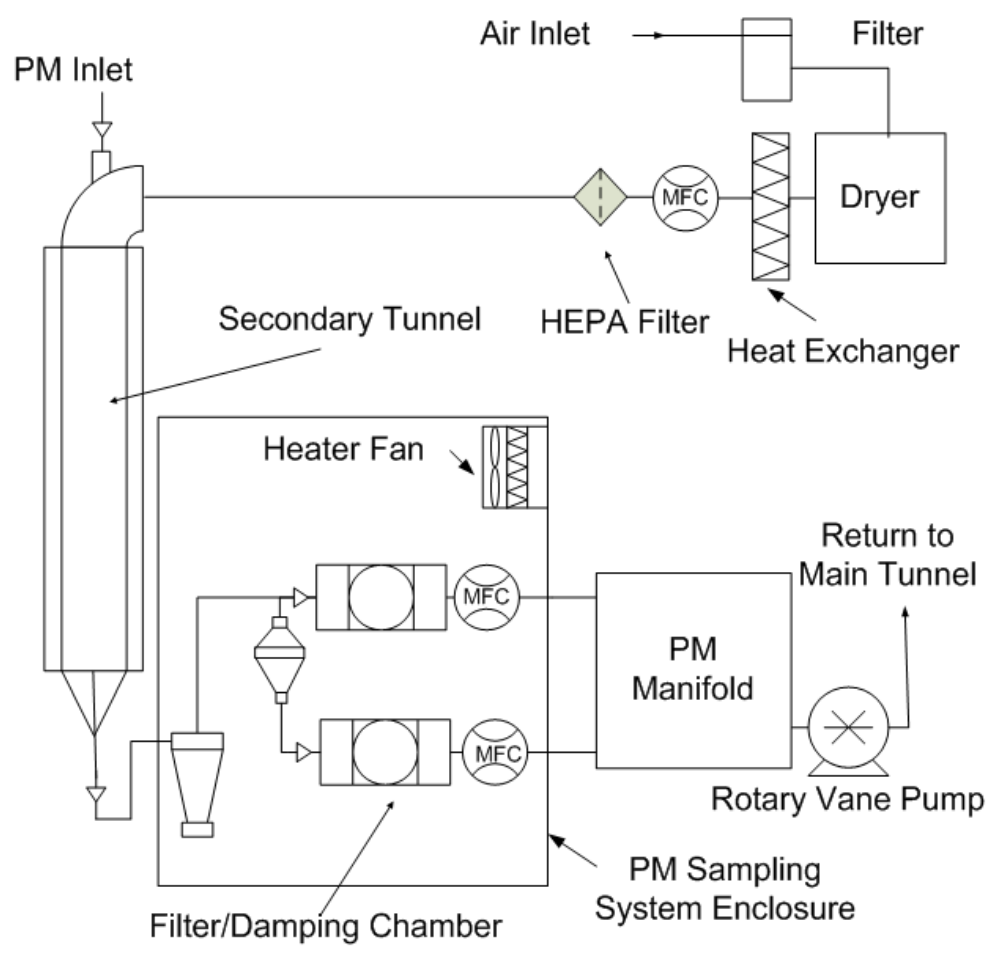

Figure 3-5 Schematic of the CFR 40 Part 1065 Regulated PM sampling system

Figure 3-6 shows the view of the temperature controlled PM sampling system with two independent streams for the clean tunnel and the dirty tunnel.

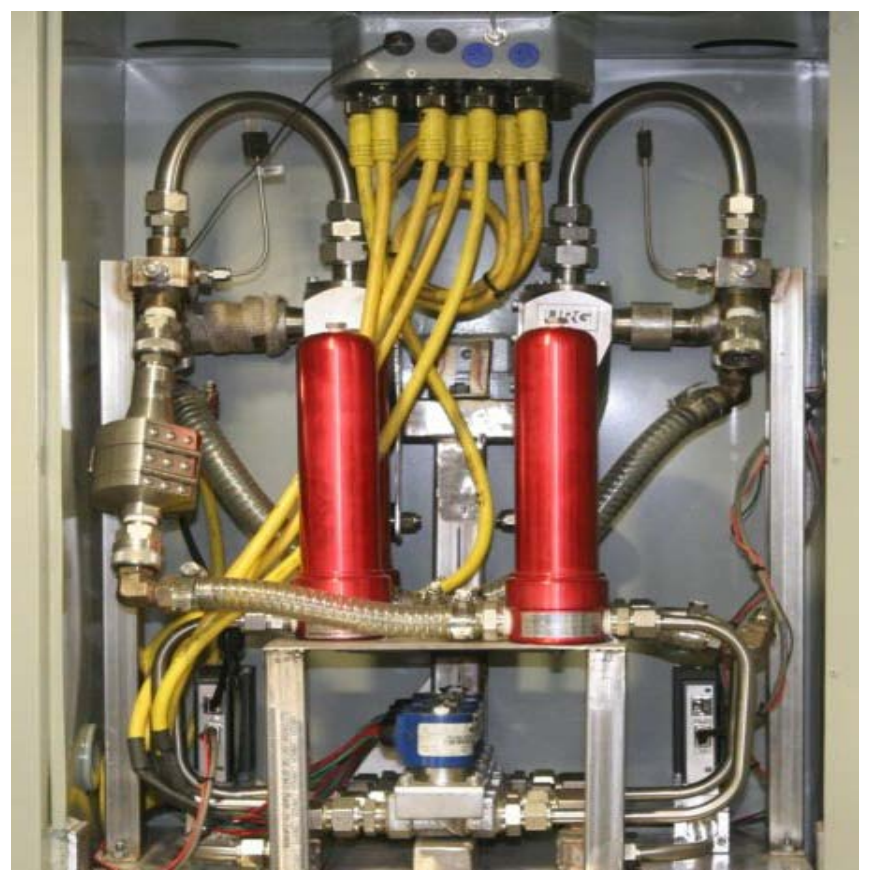

Figure 3-6 CFR 40 Part 1065 compliant PM sampling system on-board the transportable laboratory container 
The sampling system consists of the dilution air stream, which is filtered and cooled to remove moisture. The dry dilution air is then heated to $25 \pm 5^{\circ} \mathrm{C}$ as per regulations prescribed in CFR 1065 (USEPA, 2012). The conditioned secondary dilution flow is subsequently introduced to the main PM flow drawn from the primary dilution tunnel and allowed to mix in the secondary dilution tunnel. The size of the secondary dilution tunnel was determined by Simulink modeling [71]. The secondary tunnel wall is maintained at $47^{\circ} \mathrm{C}$. The flow from the secondary tunnel enters in to the PM sampling chamber through a $\mathrm{PM}_{2.5}$ cyclone and into the $47 \mathrm{~mm}$ filter holder. The PM system consists of two streams with two separate cyclones and filter holders, connected to the two different primary dilution tunnels. The PM box is also maintained at $47^{\circ} \mathrm{C}$. All flows are controlled by calibrated mass flow controllers.

\subsubsection{CVS FLOW CONTROL}

The laboratories CVS flow control is achieved through a sub-sonic venturi (SSV). The SSV installed on the transportable laboratory was supplied with 300 series Schedule 5 stainless steel pipe sections, with a nominal internal diameter of $12^{\prime \prime}$ The SSV throat diameter was 6.26". To ensure the accuracy and repeatability of SSV flow rate measurement, a straight section of Schedule 5 pipe, ten feet in length, was flanged and attached to each end of the subsonic venturi to minimize the flow wakes, or eddies, or flow circulation which might be induced by pipe bends or coarse inside walls. This particular SSV was calibrated with a reference SSV from $400 \mathrm{scfm}$ to $4000 \mathrm{scfm}$ following the procedure defined in 40 CFR Part 1065.340. The flow rate of the SSV is calculated, in real time, using the equations in 40 CFR Part 1065.640 and 40 CFR Part 1065.642 (USEPA, 2012).

\subsection{TEST VEHICLE AND ENGINE SPECIFICATIONS}

Two transit buses from the Sacramento transit agency were used for the study. The buses were CNG fueled and initially intended to be of one high mileage and one lower mileage test vehicles. However, the since the buses were all commissioned relatively at the same point into the fleet, the mileage differences were not significant. Hence, the test plan was modified to procure and test two buses with similar odometer readings. Table 2and Table 3 list the vehicle and engine specifications of the Sacramento transit buses tested in this study.

51 I $P$ a g e 
Table 2 Test vehicle specifications

\begin{tabular}{|c|c|c|c|c|c|c|c|}
\hline $\begin{array}{c}\text { Bus } \\
\#\end{array}$ & Vehicle ID & $\begin{array}{c}\text { Chassis } \\
\text { Manufacturer }\end{array}$ & GVWR & $\begin{array}{c}\text { Odometer } \\
\text { Reading }\end{array}$ & $\begin{array}{c}\text { Vehicle } \\
\text { Model } \\
\text { Year }\end{array}$ & $\begin{array}{c}\text { After- } \\
\text { treatment } \\
\text { System }\end{array}$ & Fuel \\
\hline 2834 & 1 VHGH3W2586703737 & $\begin{array}{c}\text { Daimler Bus } \\
\text { North America }\end{array}$ & 42540 & 77538 & 2008 & $\begin{array}{c}3 \text {-Way } \\
\text { Catalyst, } \\
\text { Cummins }\end{array}$ & CNG \\
\hline 2824 & 1 VHGH3W2486703602 & $\begin{array}{c}\text { Daimler Bus } \\
\text { North America }\end{array}$ & 42540 & 84994 & 2008 & $\begin{array}{c}3 \text {-Way } \\
\text { Catalyst, } \\
\text { Cummins }\end{array}$ & CNG \\
\hline
\end{tabular}

Table 3 Test vehicle engine specifications

\begin{tabular}{|c|c|c|c|c|c|}
\hline $\begin{array}{c}\text { Engine } \\
\text { Manufacturer }\end{array}$ & $\begin{array}{c}\text { Engine } \\
\text { Model }\end{array}$ & $\begin{array}{c}\text { Engine } \\
\text { Model Year }\end{array}$ & $\begin{array}{c}\text { Displacement/Power } \\
\text { (L/HP) }\end{array}$ & $\begin{array}{c}\text { Type of } \\
\text { Fuelling }\end{array}$ & $\begin{array}{c}\text { NOx/PM } \\
\text { (gm/bhp-hr) }\end{array}$ \\
\hline Cummins & ISLG 280 & 2007 & $8.9 / 280$ & $\begin{array}{c}\text { CNG } \\
\text { Stoichiometric }\end{array}$ & $0.2 / 0.01$ \\
\hline
\end{tabular}

* Values indicate the USEPA emissions standard compliance of the engine

\subsection{TEST CYCLE}

Three test cycles were used for this study. Urban Dynamometer Driving Schedule (UDDS), $45 \mathrm{MPH}$ steady state and idle test.

The UDDS cycle simulates the freeway and non-freeway operation of a heavy-duty vehicle. The UDDS and the heavy-duty federal test procedure (FTP) cycle used for engine certification were derived from the same data set. The cycle is of 1060 seconds in duration with a maximum speed of $58 \mathrm{MPH}$. The vehicle is exercised over 5.5 miles over the entire test cycle. Due to the expected low PM emissions levels from the test vehicles, the triplicate versions of the UDDS driving cycles were created. Regulated emissions were calculated over a three UDDS cycles and unregulated media were sampled over three repeats of a triple length UDDS on one media.

The $45 \mathrm{MPH}$ steady state cruise cycle was maintained for 1 hour. Idle test was lasted for 1 hour and emissions were reported as grams/sec of idle. Figure 3-7 shows the 16.5 mile long and 53 minute duration triple-UDDS cycle. 


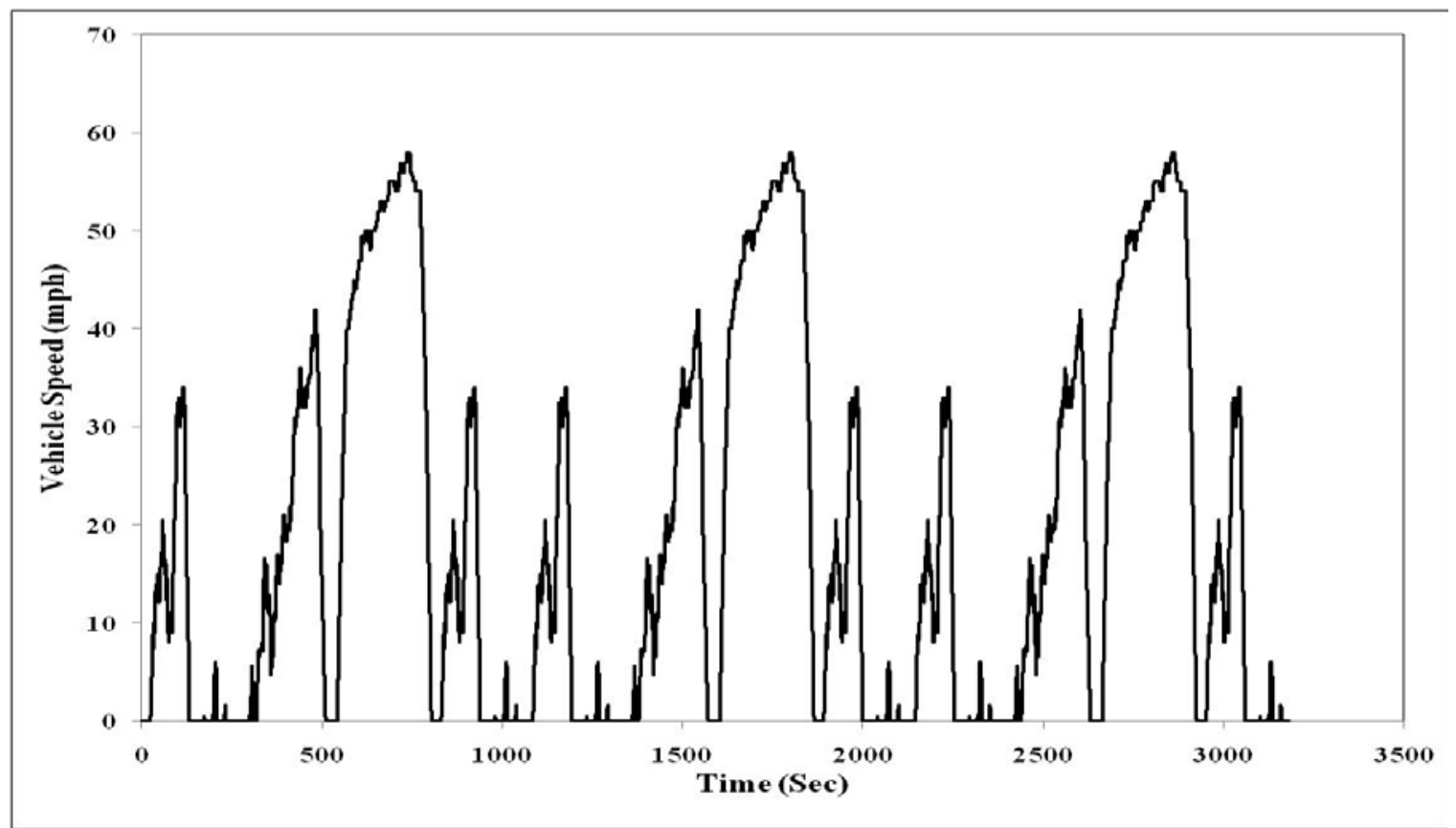

Figure 3-7 Speed Vs. Time trace of triple length UDDS cycle

\subsection{THERMODENUDER PM SAMPLING SYSTEM}

Thermodenuder PM sampling system was designed and built at WVU to perform high volume sampling of thermally denuded and non-denuded PM samples on filter media. The system was designed to contain 4 thermal denuders, with independent flow control using mass flow controllers to maintain a flow rate of $50 \mathrm{lpm}$. Samples downstream of the denuders were collected onto separate $47 \mathrm{~mm}$ T60A20 filters. The sampling system also contained the 5th sampling stream for the non-denuded sample collection. This stream was designed as a high volume sampling stream with flow rate of $2001 \mathrm{pm}$, controlled by a calibrated critical flow orifice. Thermal denuders were air cooled by circulating compressed air through its cooling stream. Figure 9 and Figure 10 show the thermodenuder sampling system setup. 


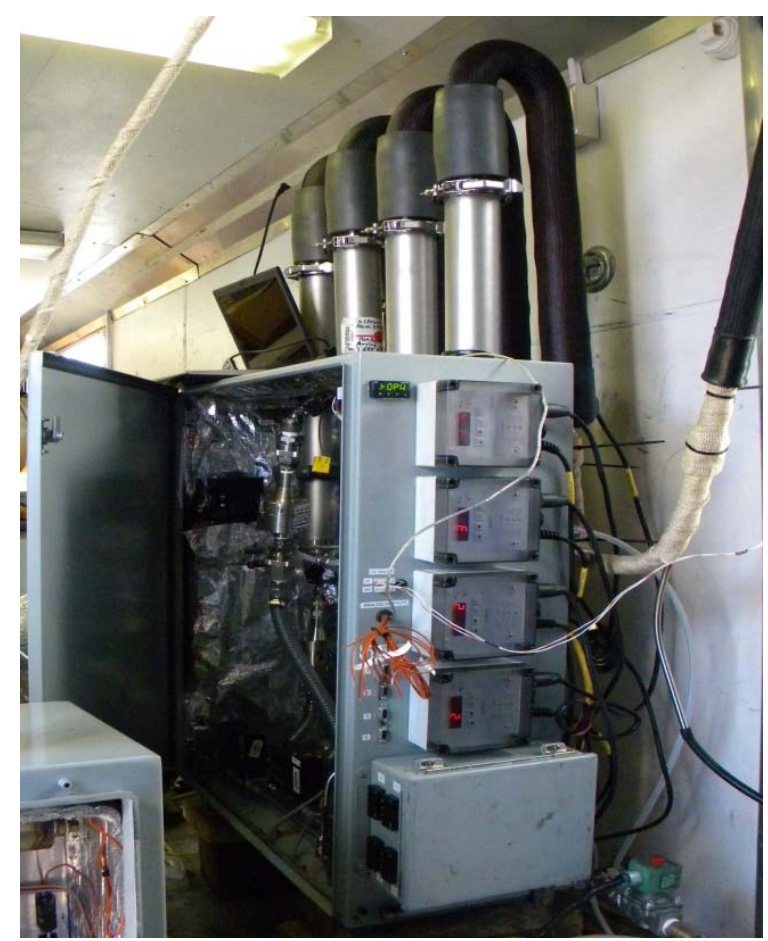

Figure 3-8 Outside view of the thermodenuder sampling system

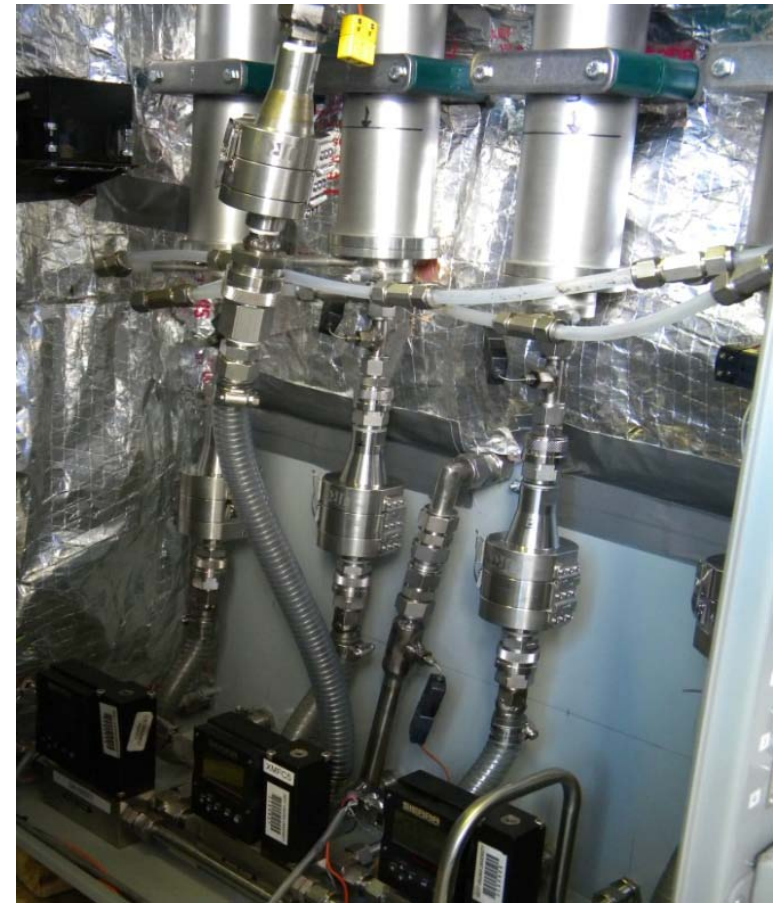

Figure 3-9 Inside view of thermodenuder sampling system

National instruments DAQ NI-USB6009 was used to record flow, temperature, pressure and also to control the set points of the mass flow controllers. National Instruments Lab View software was used to create the data acquisition program for the sampling system. The system 
initiation was linked to the container in order to sync data collection with the main container DAQ system.

\subsection{PARTICULATE MATTER SIZING SETUP}

The study employed the TSI EEPS (TSI Model 3090) for transient particle size distribution measurement and the TSI EAD (TSI Model 3070A) to monitor the instantaneous particle concentration with respect to an aerosol parameter called total aerosol length. Both the EEPS and the EAD were setup up for sampling from the CVS dilution tunnel. This method of sampling was chosen over partial flow dilution of raw exhaust using ejector pumps in order to better correlate the gravimetric PM and the particle size distribution and concentration formed as a result of dilution within the CVS. Further the PM size distribution and concentration data could also be associated with toxicity data as dilution conditions for both sampling stream are the same. As preliminary procedures sample flow rates of the EEPS were verified with a standard flow meter, and the operation of the instrument was checked against the standard TSI SMPS (TSI Model 3936). The instrument was cleaned and electrometers were zeroed before the commencement of the study.

Since the EEPS was sampling directly from the CVS tunnel, the only applicable dilution ratio is based on the exhaust flow ( $\left.V_{\text {exhaust }}\right)$ and the total CVS flow $\left(V_{\text {mix }}\right)$; hence, is a function of engine operation.

$$
D R=\frac{\dot{V}_{\text {mix }}}{\dot{V}_{\text {exhaust }}}=\frac{\mathrm{mol} / \mathrm{sec}}{\mathrm{mol} / \mathrm{sec}}
$$

\section{Equation 8}

Due to the lack of accurate exhaust flow measurements, the dilution ratio has been approximated based on a simplified carbon balance, assuming stoichiometric combustion (Eq. $3.3)$, methane $(\mathrm{CH} 4)$ and ideal air $(21 \% \mathrm{O} 2,79 \% \mathrm{~N} 2)$ as the sole reactants as well as no carbon content $(\mathrm{CO} 2, \mathrm{CO}, \mathrm{HC})$ within the dilution air, and the continuous measurement of $\mathrm{CO} 2$ within the CVS tunnel. According to Equation 3.3 for each mole of $\mathrm{CO} 2$ produced one mole of $\mathrm{CH} 4$ is being consumed. Therefore, multiplying the amount of CO2 measured at the CVS sample plane 
with the total flow through the CVS system (Vmix) gives the molar rate of $\mathrm{CH} 4$ burned as shown in Equation 9.

$$
\begin{gathered}
\mathrm{CH}_{4}+2\left(\mathrm{O}_{2}+3.76 \mathrm{~N}_{2}\right) \stackrel{\text { yields }}{\longrightarrow} \mathrm{CO}_{2}+2 \mathrm{H}_{2} \mathrm{O}+7.52 \mathrm{~N}_{2} \\
\mathrm{CH}_{4}(\mathrm{~mol} / \mathrm{sec})=\frac{\mathrm{CO}_{2}(\mathrm{ppm})}{10^{6}} * \dot{V}_{\text {mix }}
\end{gathered}
$$

Equation 9

Equation 10

Further, using the theoretical air-fuel ratio (AFRmolar) on a molar basis (9.52 for $\mathrm{CH} 4$ ), calculated from the stoichiometric reaction equation (Eq. 3.3) the molar rate of intake air flow and exhaust flow can be estimated based on (Eq. 3.5) and (Eq. 3.6), respectively.

$$
\begin{gathered}
\dot{V}_{\text {intake }}(\boldsymbol{m o l} / \text { sec })=\boldsymbol{C H}_{4}(\mathbf{m o l} / \text { sec }) * \boldsymbol{A F} \boldsymbol{R}_{\text {molar }} \\
\dot{V}_{\text {exhaust }}(\mathrm{mol} / \mathrm{sec})=\dot{V}_{\text {intake }}(\mathrm{mol} / \mathrm{sec}) * C H_{4}(\mathrm{~mol} / \mathrm{sec})
\end{gathered}
$$

Equation 11

Equation 12

\subsection{UNREGULATED EMISSIONS SAMPLING SYSTEM}

The samples of unregulated emissions were drawn at the second sampling section downstream of the regulated emissions sampling section. The unregulated emissions sampling system consisted of various sampling trains to sample different emissions which are described in the following sections.

\subsubsection{CARBONYLS}

The carbonyl sampling system consisted of a heated probe and a heated sampling line whose temperatures were maintained at $240^{\circ} \mathrm{F}\left(115.5^{\circ} \mathrm{C}\right)$ to prevent water condensation. A flow of $2 \mathrm{lpm}$ was maintained using a calibrated critical flow orifice and a vacuum pump. The sample was collected into cartridges packed with silica gel coated with acidified 2, 4- 
dinitrophenylhydrazine (DNPH). The cartridges were then shipped to a CARB MLD laboratory for subsequent analysis.

\subsubsection{POLYCYCLIC AROMATIC HYDROCARBON (PAH) SAMPLING SYSTEM}

PAH was sampled from the tunnel into PUF/XAD cartridges. Sample from dilution tunnel was drawn at $200 \mathrm{lpm}$. The flow control was achieved through a critical flow orifice. The sample is admitted into the sampler through a copper tube and into a manifold which contains the housing for the PUF/XAD cartridge. Figure 11 shows the schematic of the PUF/XAD cartridge housing. The housing holds the PUF/XAD cartridge and a 70mm T60A20 filter used to capture the PM bound organic compounds. The pumps are initiated manually with the start of the test.

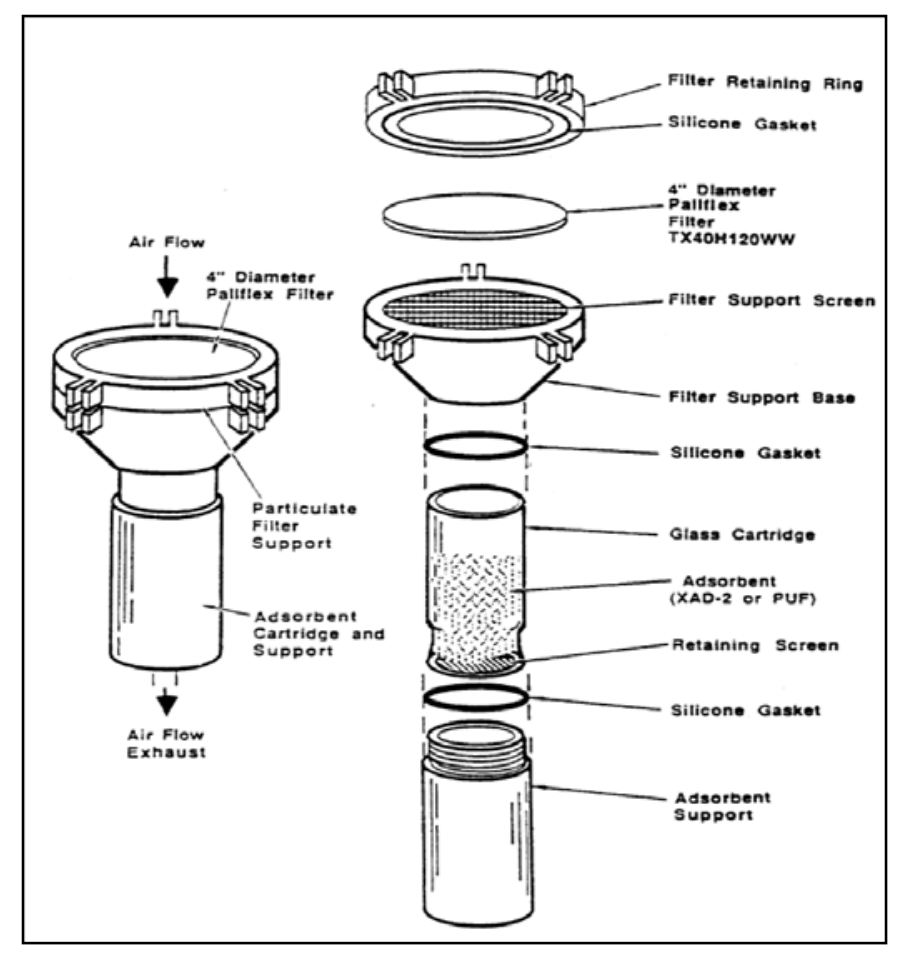

Figure 3-10 Schematic of WVU PUF/XAD cartridge housing

\subsubsection{VOC CANISTER SAMPLING SYSTEM}

VOCs were collected into a steel canister for laboratory analysis using a critical flow orifice system provided by CARB Monitoring Laboratory Division. The canister had a capacity of 6.0L at atmospheric condition. The steel canisters used to collect dilute exhaust were completely evacuated, and a vacuum of $10 \mathrm{~mm} \mathrm{Hg}$ was present inside the canister prior to the start of the test. Sample was collected below atmospheric pressure to prevent water condensation. The 
vacuum inside the canister was used to draw the sample from the tunnel, through the critical flow orifice.

\subsubsection{CYCLONIC PARTICLE CLASSIFIER}

Sampling of unregulated emissions included gravimetric analysis of particulate matter in size fractions of $10 \mu \mathrm{m}, 2.5 \mu \mathrm{m}$ and $1 \mu \mathrm{m}$. This was accomplished by using cyclone classifiers (URG 16.7 lpm cyclone). Cyclone classifier makes use of vertical flow inside a cylindrical or conical chamber to separate particles depending on the flow rate. A double vortex flow is induced in the conical body of the cyclone by introducing the sample tangentially at the top where flow spirals down along the wall, which then reverses and spirals through inner core to exit the chamber. Particles with sufficient inertia impact on the cyclone wall as they cannot follow the streamlines of the flow exiting the chamber. The various size fractions of PM were sampled directly from the primary dilution, as the tunnel was built to a single stage PM sampling specification. The samples were collected on a $47 \mathrm{~mm}$ Teflon coated glass fiber filters (T60A20). The flow through the cyclone was controlled using mass flow controllers.

\subsubsection{GAS BAG SAMPLING}

Tedlar bags were used to collect samples of dilute exhaust gas and dilution air during each emissions test. The bag samples were analyzed at the end of every test to perform background correction of the regulated emission constituents. The dilute exhaust bag served as a QA/QC check point when compared with the continuous sample.

\subsection{INSTRUMENTATION CONTROL AND DATA ACQUISISTION}

A software application developed in-house controlled the power absorbers and the data acquisition system. The program acquired the raw data ( $A D C$ codes) and a reduction program converted the raw data into proper engineering units using respective calibration curves.

\subsection{MEDIA}

Different types of media were used to collect samples for speciation and gravimetric analysis during the test procedure. The media were conditioned, weighed and handled as per

58 | P a g e 
the regulations outlined in 40 CFR, Part 1065. A list of species and the media used to collect them is given in the Table 4.

Table 4 List of unregulated species and sample media

\begin{tabular}{|l|c|}
\hline \multicolumn{1}{|c|}{ Species } & Collecting Media \\
\hline Volatile Organic Compound (VOC), & VOC Steel Canister \\
\hline PAH, n-PAH & $\begin{array}{c}\text { TX 40 70 mm Filter + } \\
\text { PUF/XAD Cartridge }\end{array}$ \\
\hline Aldehydes & DNPH Cartridge \\
\hline PM2.5 Metals/Ions & Teflo (47mm) Filter \\
\hline PM1.0/PM 2.5 Gravimetric & T60A20 (47mm) Filter \\
\hline
\end{tabular}

\subsection{MEDIA CONDITIONING AND WEIGHING}

The media used in collecting PM for gravimetric analysis were conditioned and preweighed in the WVU engine research center facility before transporting them to the test site. The filters were conditioned in an environmentally controlled chamber (Class 1000) maintained at $70^{\circ} \mathrm{F}$ with a relative humidity of $50 \%$, for at least 1 hour and not more than 80 hours before being weighed according to 40 CFR, Part 1065 specifications. The $70 \mathrm{~mm}$ filters were placed in the glass petri dishes and $47 \mathrm{~mm}$ filters were placed in plastic analyslides, with their lids closed but not sealed in order to prevent dust from accumulating on the media while allowing for humidity exchange. Two reference filters were conditioned with the test filters and placed in the environmental chamber in order to account for change in the filter weight due to fluctuation in humidity. The reference filters were weighed before and after any set of media were weighed to ensure that the conditions in the environmental chamber were stable.

The filters were pre-weighed after a stabilization period of 24 hours using a Metler -Toledo Microbalance. The balance was interfaced with a computer in which the filter weights were logged for future reference and use. The filters were conditioned for several hours in the chamber before the petri-dishes were sealed and packed in padded envelopes for shipping to the test site. 


\subsection{MEDIA SHIPPING AND TRACKING}

The petri-dishes containing the filter media were sealed and placed in padded envelopes after they had been pre-weighed. The set of envelopes containing media required for one test were placed together to aid in quick loading of the media into the sampling system. The sealed media were transported overnight to the test site in coolers filled with frozen water pouches. After the media was received at the test site it was placed in the conditioning room until used. A media tracking application was developed to identify the media with the test sequence and run number. The tracking tool also aided in QA/QC protocol. The used media were placed back into their respective petri-dishes in the conditioning room and were tracked before shipping to the location, where they were analyzed. 


\section{CHAPTER 4 - EMISSIONS TESTING PROCEDURE}

\subsection{LABORATORY SET-UP}

The chassis dynamometer which is built onto a flat bed trailer was set-up on a flat surface and leveled to prevent variation in the vehicle's inertial loading which is simulated using rotating flywheels. The emissions measurement container which houses the analyzers, dilution tunnel, dynamometer control and signal conditioning devices was placed close to the chassis dynamometer. This reduced the length of exhaust tubing between the tail pipe and the dilution tunnel in turn reducing thermophoretic and other losses of particulate matter in the transfer tube. The blower was placed at the end of the dilution tunnel and a flexible air duct was used in connecting the tunnel to the blower. HEPA filters were installed to the inlet manifold of the dilution tunnel. After all the connections were made to the dilution tunnel the instrument trailer was prepared for testing.

\subsection{LABORATORY CHECKS}

Initial laboratory set-up procedures include complete measurement system verification followed by calibration. All required system verifications are performed as per requirements stated in 40 CFR, Part 1065, Subpart D. The measurement container is equipped with the Horiba Mexa 7200 Motor Exhaust Gas Analyzer, which is capable of automatically performing the required analyzer verification tests. The verification procedure and pass criteria of the tests were in accordance to the provisions described in 40 CFR, Part 1065, Subpart D. Table 6 lists the complete set of analyzer verification checks performed on field prior to the commencement of the testing. Table 5 lists the complete set of leak checks performed on the gaseous and PM measurement systems.

Table 5 Gaseous and PM measurement system verification checks

\begin{tabular}{|c|c|}
\hline Leak Checks & Pass Criteria \\
\hline Leak and Delay Time Check (all analyzers) & $\begin{array}{c}\text { Within } \pm 5 \% \text { over } 30 \text { sec } \\
\text { interval }\end{array}$ \\
\hline PM System 1 Leak Check & \\
\hline PM System 2 Leak Check & \\
\hline
\end{tabular}


Table 6 Gaseous analyzer verification checks

\begin{tabular}{|c|c|}
\hline Analyzer Checks & Pass Criteria \\
\hline THC1 Hang-up & \\
\hline THC2 Hang-up & Within $\pm 1 \%$ \\
\hline $\mathrm{CO}(\mathrm{L}), \mathrm{CO}_{2}$ Interference Check & Within $\pm 2 \%$ \\
\hline THC, $\mathrm{O}_{2}$ Interference Check & Within $\pm 1 \%$ \\
\hline $\mathrm{CO}_{2}$ Quench $\mathrm{NO}_{\mathrm{x}} 1$ & Within $\pm 1 \%$ \\
\hline $\mathrm{CO}_{2}$ Quench $\mathrm{NO}_{\mathrm{x}} 2$ & Within $\pm 1 \%$ \\
\hline $\mathrm{H}_{2} \mathrm{O}$ Quench $\mathrm{NO}_{\mathrm{x}} 1$ & Within $\pm 1 \%$ \\
\hline $\mathrm{H}_{2} \mathrm{O}$ Quench $\mathrm{NO}_{\mathrm{x}} 2$ & $\mathrm{PFCH}_{4}>0.85$ and $\mathrm{PFC}_{2} \mathrm{H}_{6}<0.02$ \\
\hline Non-Methane Cutter $\mathrm{Efficiency}$ & \\
\hline
\end{tabular}

\subsection{MASS FLOW CONTROLLER CALIBRATION}

Mass flow controllers were used in controlling the flow through cyclonic particle classifier, TPM flow through the filter and various other unregulated emissions sampling systems. The calibration was performed against a Laminar Flow Element supplied by Meriam Flow Measurement Devices. Meriam provides a calibration equation and co-efficient for each LFE which is obtained through calibration involving a flow meter that is traceable to NIST standards. A five point calibration was performed on the MFCs between fully open and fully closed position.

Differential pressure across the LFE and absolute pressure was measured using a Heise pressure reader and the temperature was measured using a Fluke Temperature calibrator. The actual flow measured through the LFE was converted to standard flow by 40 CFR, Part 1065 specified standard condition of $20^{\circ} \mathrm{C}$ and $101.1 \mathrm{kPa}$.

\subsection{CVS-SSV DILUTION TUNNEL VERIFICATION}

The CVS system was verified by injecting a known quantity of propane into the primary dilution tunnel while CVS-SSV system operating. The concentration of the propane was determined using a pre-calibrated HFID analyzer and the mass of propane injected was 
measured by the flow data and the density of propane. The propane injection test helped in determining leak in the tunnel and any discrepancy in the flow measuring device (CVS-SSV system).

The method uses a propane injection kit with a critical flow orifice meter to accurately measure the amount of propane injected into the tunnel. The flow rate of propane through the orifice meter is determined by measuring the inlet temperature and pressure using Equation 13.

$$
q=\frac{A+(B * P)+\left(C * P^{2}\right)}{\sqrt{460+T}}
$$

Equation 13

Where:

$\mathrm{q}=$ flow rate through orifice in SCFM

A, B, C = Calibration coefficients of the orifice provided by the propane injection kit manufacturer (Horiba)

$\mathrm{P} \quad=$ Absolute orifice inlet pressure, in psia

$\mathrm{T} \quad=$ Orifice inlet temperature in ${ }^{\circ} \mathrm{F}$

The total flow through CVS is given by Equation 14

$$
Q=\frac{\dot{V}}{60} * t
$$

Equation 14

Where:

Q $\quad=$ Total volume in SCF

$\mathrm{V}=$ = Flow rate in SCFM measured by the subsonic venture of the CVS tunnel

$\mathrm{t} \quad=$ Time interval in seconds, usually 300 seconds

The calculated sample concentration was determined by Equation 15 


$$
C_{c a l c}=\left(\frac{q}{Q} * 10^{6}\right) * 3
$$

Equation 15

The system error is given by Equation 16

$$
\text { Error }=\left(\frac{C_{a b s}}{C_{c a l c}}-1\right) * 100
$$

Equation 16

Where:

$\mathrm{C}_{\mathrm{obs}}=$ Measured concentration of the injected propane by HFID analyzer

If the error is greater than $2 \%$, then the cause for discrepancy was found and corrected. The error could be due to various reasons such as leaks before the sampling plane, leaks after the sampling plane and improper analyzer calibration. Three repeatable propane injections within a difference $0.5 \%$ of each other is required to pass the dilution tunnel verification test.

\subsection{TEST PROCEDURE}

Before mounting the vehicle on the chassis dynamometer the appropriate flywheel combination was determined and locked in place to simulate the inertial load of the vehicle. The inertia setting for the bus was equal $34450 \mathrm{lbs}$. The outer rear wheel on the drive axle is removed and fitted with hub adapters which are later connected to the face plate. The vehicle was backed onto the dynamometer and the vehicle drive axle which drives the flywheel assembly and power absorbers were connected through a hub adapter. The vehicle was leveled with the drive axle and the tires were checked for any distortion as it would add to the vehicle loading. The vehicle exhaust was now connected to the dilution tunnel via insulated transfer tubes. The vehicle was chained down to the dynamometer bed as a safety measure.

The vehicle was made to run at a high speed after being mounted on the dynamometer to warm the lubricating oil in the differentials. This was done to reduce additional load on the vehicle due to highly viscous oil. During warming up of the differentials the gas analyzers were zero-spanned with blower operating at set-point. The driver interface speed monitor and 
communication head sets were put in place to aid the driver in following the scheduled drive cycle. A dummy test was conducted by making vehicle to run over the scheduled drive cycle with dummy media loaded in the tunnel to check whether the gas analyzers operated within the range for which they were calibrated and to check the flow through the mass flow controllers. If the analyzers pegged or measured below the range then they were recalibrated with proper span gas and the mass flow controllers were checked for any malfunction. After the warm up run the vehicle was shut down and allowed to soak for twenty minutes. During the soak time the official media required for various sampling were loaded in their respective holders and mounted onto the tunnel. The media loading was carried out in the controlled chamber to avoid accumulation of dust or other debris, a set of field blanks were maintained to study the effect of transportation on the used and unused media.

This study was unique for the fact that the work plan required adequate mass collection on the thermally denuded and non-denuded filters for toxicity and mutagenicity assays. The toxicity analysis required $1 \mathrm{mg}$ loading on the filters and the mutagenicity assays required $0.5 \mathrm{mg}$ loading on the filters. However, natural gas PM consisting predominantly of organic fraction, would be completely devoid of any mass in the thermally denuded sample. Hence, the test matrix was decided to be based on the mass loading on the filters of the non-denuded stream.

Initial test runs were conducted to infer the PM mass emissions from the test vehicles. Due to the lack of accurate weighing capabilities on field, the number of hours of cycle repetitions was decided based on the initial test runs for each cycle. Table 7 below summarizes the number of repetitions of the individual cycle to attain adequate mass loading.

Table 7 Cycle repetitions for PM mass loading

\begin{tabular}{|l|c|c|}
\hline Test Cycle & $\begin{array}{c}\text { Cycle Duration } \\
\text { (Min) }\end{array}$ & $\begin{array}{c}\text { Number of } \\
\text { Repetition }\end{array}$ \\
\hline 3x UDDS & 53 & 10 \\
\hline Idle & 60 & 13 \\
\hline $45 \mathrm{MPH}$ & 60 & 12 \\
\hline
\end{tabular}


Vehicles were repeatedly exercised over their respective drive cycles for the stipulated number of testing hours to collect PM onto the filters. The filters were not replaced and mass from consecutive testes were repeatedly loaded onto the same filters. Decision to extend the test matrix, if required were made based on approximate and unconditioned weighing of the filters at the Stockton, CARB facility. Unregulated sampling streams were sampled over three repetitions of each cycle. Tunnel blanks and system blanks were collected for all sample streams at the end of each testing day. Figure 4-1 and Table 8 summarizes the different special sampling streams with their respective sampling instruments and flow rate settings.

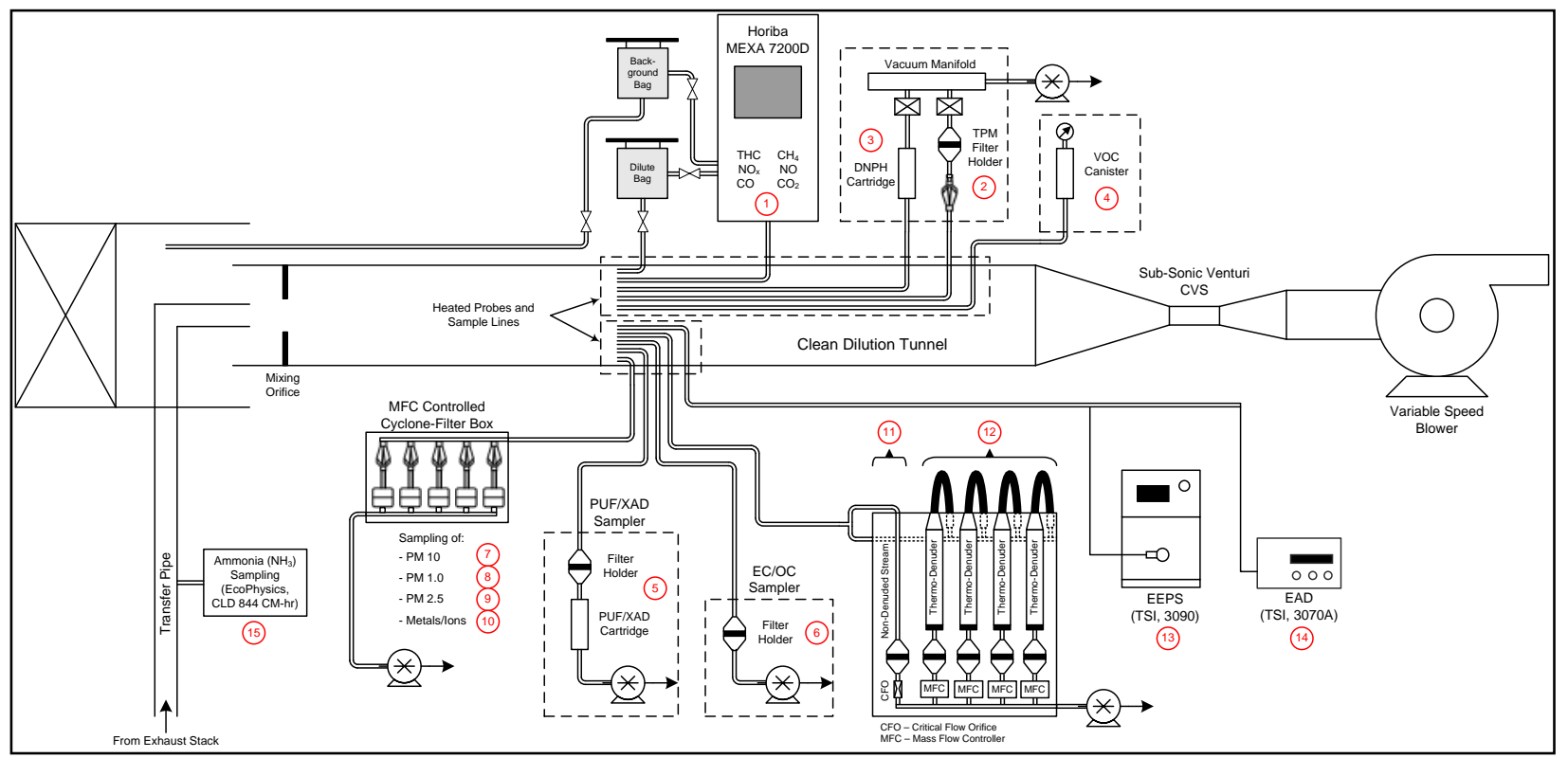

Figure 4-1 Schematic of CVS and sampling streams

Table 8 Summary of sampling media, sampling devices and methods used to analyze unregulated exhaust species

\begin{tabular}{|c|l|c|c|}
\hline ID & \multicolumn{1}{|c|}{ Sample } & Media Type & Flow rate/ Flow Control \\
\hline \multicolumn{2}{|c|}{ Unregulated Sampling } \\
\hline 3 & Carbonyls & DNPH cartridge & 2 lpm, Critical flow orifice \\
\hline 4 & VOC & Steel Canister & $\begin{array}{c}\text { Critical flow orifice provided } \\
\text { by MLD }\end{array}$ \\
\hline 5 & PAH & PUF/XAD and 90mm T60A20 & 200 lpm, Critical flow orifice \\
\hline 6 & PM 2.5 EC/OC & $\begin{array}{c}\text { Pre-fired Quartz (WVU Critical flow } \\
\text { Cyclone Sampling) }\end{array}$ & $\begin{array}{c}16.7 \text { lpm (2.5 micron URG } \\
\text { Cyclone) }\end{array}$ \\
\hline 9 & PM 2.5 Ions & $\begin{array}{c}\text { Teflon (WVU Critical flow Cyclone } \\
\text { Sampling) }\end{array}$ & $\begin{array}{c}16.7 \text { lpm (2.5 micron URG } \\
\text { Cyclone) }\end{array}$ \\
\hline
\end{tabular}




\begin{tabular}{|c|l|c|c|}
\hline 10 & PM 2.5 Metals & $\begin{array}{c}\text { Teflon (WVU Critical flow Cyclone } \\
\text { Sampling) }\end{array}$ & $\begin{array}{c}16.7 \text { lpm (2.5 micron URG } \\
\text { Cyclone) }\end{array}$ \\
\hline 13 & Particle Size Dist. & Transient EEPS, EAD & 10 lpm \\
\hline ID & Sample & Media Type & Flow rate/ Flow Control \\
\hline \multicolumn{5}{|c|}{ Toxicity Sampling } \\
\hline 11 & $\begin{array}{l}\text { Toxicity stream } \\
\text { with Volatiles }\end{array}$ & $\begin{array}{c}47 \mathrm{~mm} \text { T60A20 } \\
200 \mathrm{lpm} \text {, Critical flow orifice }\end{array}$ \\
\hline 12 & $\begin{array}{l}\text { Toxicity stream } \\
\text { w/o Volatiles }\end{array}$ & $\begin{array}{c}47 \mathrm{~mm} \text { T60A20 through the } \\
\text { Thermodenuder System }\end{array}$ & $\begin{array}{c}50 \mathrm{l} / \mathrm{min} / \text { denuder stream, } \\
\text { mass flow controller }\end{array}$ \\
\hline 11 & ROS Tox & $47 m m$ T60A20 & $2.5 \mathrm{scfm}$, mass flow controller \\
\hline 11 & Mutagencity & $47 \mathrm{~mm}$ T60A20 & $2.5 \mathrm{scfm}$ mass flow controller \\
\hline
\end{tabular}

\subsection{EMISSIONS CALCULATIONS}

\subsubsection{EQUATIONS TO CALCULATE DISTANCE SPECIFIC MASS OF UNREGULATED SPECIES}

The equation used in calculating the distance specific emissions of unregulated components is similar to the equation used in calculating the regulated emissions. The unregulated species analyses were carried out by multiple institutions as tabulated in Table X.

The chemical speciation analysis was performed by calculating the total volumetric flow through the tunnel.

$$
V_{\text {Tunnel }}=\left(V_{\text {mix }}+V_{\text {min } i}+V_{\text {PUF / XAD }}+V_{\text {DNPH }}+V_{V O C}+V_{\text {Denuder }}+V_{P M 1.0}+V_{P M 2.5}+V_{P M 10}\right)
$$

Equation 17

Where:

$$
\begin{array}{ll}
V_{\text {Tunnel }} & =\text { Total tunnel flow before removing the samples, in SCF } \\
V_{\text {mix }} & =\text { Total flow through the tunnel with samples removed for analysis, in SCF } \\
V_{\text {mini }} & =\text { Total flow through the secondary dilution tunnel, in SCF } \\
V_{\text {PUF } / X A D} & =\text { Total flow through the PUF/XAD cartridge, in SCF } \\
V_{\text {DNPH }} \quad=\text { Total flow through the DNPH cartridge, in SCF }
\end{array}
$$




$$
\begin{array}{ll}
V_{\text {VOC }} & =\text { Total flow collected into VOC canisters, in SCF } \\
V_{\text {Denuder }} & =\text { Total flow from denuded and non-denuded stream, in SCF }
\end{array}
$$

$\mathrm{V}_{\mathrm{PM} 1.0}, \mathrm{~V}_{\mathrm{PM} 2.5}, \mathrm{~V}_{\mathrm{PM} 10.0}=$ Total flow through the respective cyclones, in SCF

The tunnel concentration of unregulated species is calculated by the following equation.

$$
\text { Species }_{\text {TestUncorrected }}=V_{\text {tunnel }} * \text { Species }_{\text {sample }} * 10^{-6}
$$

Equation 18

Where,

Species $_{\text {TestUncorrected }}$ : Tunnel concentration of individual species without correcting for background concentration (gms).

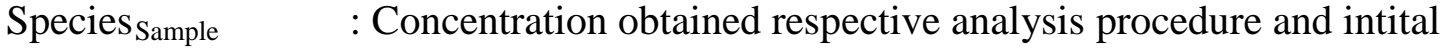
data processing from SWRI ( $\quad$ ).

$V_{\text {tunnel }} \quad$ : As calculated in Equation $17\left(\mathrm{~m}^{3}\right)$

$$
\text { Sepcies }_{\text {TestUncorrected }}^{\text {Dis tan ceSpecific }}=\frac{\text { Species }_{\text {TestUncorrected }}}{\text { TotalDis tan ce }}(\mathrm{g} / \text { mile })
$$

Equation 19 


\section{CHAPTER 5 - RESULTS AND DISCUSSIONS}

\subsection{REGULATED EMISSIONS}

A summary of the regulated emissions from the two buses tested over the three different test cycles are shown in Figure 5-1 and Figure 5-2. Figure 5-1 represents the regulated emissions as work specific and Figure 5-2 represents the regulated emissions as distance specific. The emissions from the chassis laboratory are usually represented as distance specific, however due to the availability of hub work (ahp-hr) the results are also represented in a work specific format. The work specific results can be used as an indication of the vehicles emissions limits with respect to its certification values. Results are presented as averaged results of number of tests that satisfy a $\mathrm{COV}$ of $5 \%$ for $\mathrm{CO}_{2}$ emissions. The error bars indicate the maximum and minimum emission values of the averaged tests. The $\mathrm{CO}_{2}$ are scaled down by a factor of 100 for ease of data plotting. Idle emissions are plotted as $\mathrm{g} / \mathrm{sec}$ and scaled by a factor of 100 for ease of plotting. The hydrocarbon (HC) plotted in the chart represents THC. The presence of an oxidation catalyst reduced the concentration of NMHC to very low levels, and hence the THC concentrations were very close to the methane concentrations. THC values are plotted to avoid reporting very small and in some cases negative NMHC values.

Regulated emissions were sampled over a triplicate length UDDS cycle. The 2010 compliant ISL G-280 Cummins CNG engines are stoichiometrically fueled, 3-way catalyst equipped engines. The 3-way catalyst is effective in reducing NOx emissions with stoichiometric fuelling. Axle-work specific NOx emissions from the vehicles were close to certification limit of 0.20 $\mathrm{g} / \mathrm{bhp}$-hr. However, it is to be noted that certification values are calculated to work available at flywheel, and the chassis dynamometer calculates work available at the wheels. Engine work would be significantly higher than the work available at wheels due to transmission inefficiencies and loading from auxiliary systems such as air compressors.

NOx emissions from bus 2 were 30\% lower than bus 1 emissions over the UDDS cycle. This could be due to the differences in catalytic activity in reducing NOx emissions. Further the difference in catalytic activity is also observed in the emissions of CO and THC from the two 
buses. Bus 2 was observed to emit 49\% lower CO emissions than bus 1, similarly the THC emissions from bus 2 were $46 \%$ lower than bus 1 . It is also to be noted that a $6 \%$ difference in CO2 emissions over the UDDS cycle between the two buses indicate a very similar fuel consumption. The PM emissions from bus 2 were 52\% greater than of bus 1 . CNG vehicles are usually characterized by low PM mass emissions with higher organic carbon or volatile carbon fraction of PM. A major portion of the PM emissions from a natural gas vehicle is attributed to entry of lubrication oil into the combustion chamber. The observed results could indicate a higher lubrication oil effect on bus 2 in comparison to bus 1.

NOx emissions were close to detection limits, during the $45 \mathrm{MPH}$ steady state cycles, due to a steady fuelling rate. Stoichiometric engine's fuelling is usually characterized by slight oscillations in air-fuel ratio known as dithering. Dithering aids in the simultaneous reduction of NOx, CO and hydrocarbons. The frequency and amplitude of dithering, directly affects the reduction efficiencies of the compounds, since the optimum reduction window is continuously changing. The dithering procedure is better controlled and well optimized during steady state operation as throttle demand and load variations are minimal. Hence, the resulting dithering frequencies and amplitude during cruise mode operation results in the best possible efficiencies for NOx, CO and THC emissions. As a result the $45 \mathrm{MPH}$ cruise mode driving cycle resulted in low NOx emissions of 0.096 gms/ahp-hr and 0.157 gms/ahp-hr for bus 1 and bus 2 respectively. 


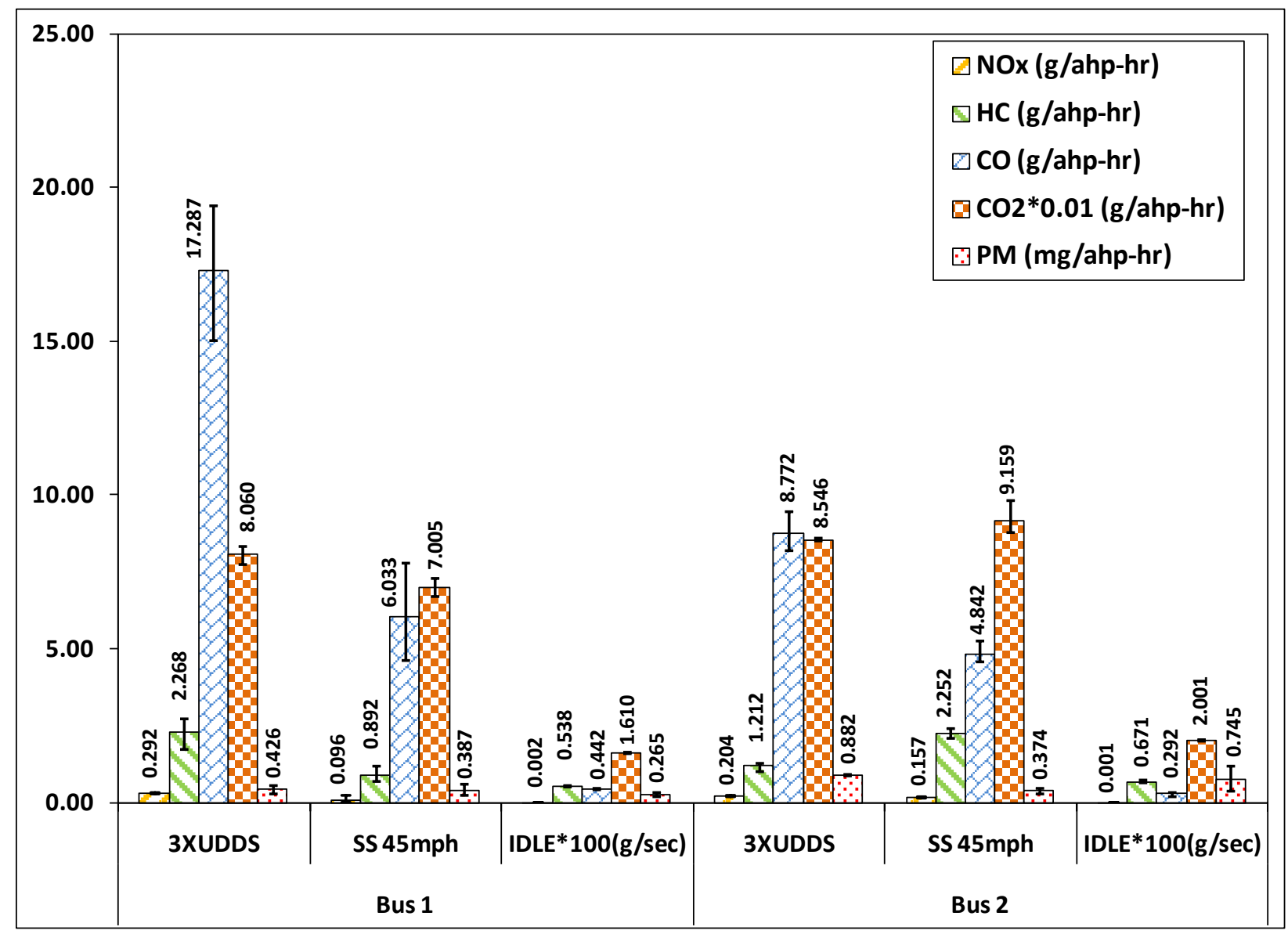

Figure 5-1 Axle work-specific regulated emissions summary of test vehicles over three different driving cycles

The PM mass emission on idle mode from bus 2 was $64 \%$ higher than bus 1 . This could be attributed to the increased lubrication oil consumption by engine in bus 2 . Although the total miles travelled by both buses are very similar, differences in day to day duty cycle as result of difference in operating routes could possibly affect emissions rates from engines, specifically PM. Vehicles operating on routes characterized by denser traffic and more frequent stops would operate extensively on lower loads and subject to frequent accelerations and decelerations which could influence oil consumption rate of an engine and result in higher mass emissions rates of PM. 


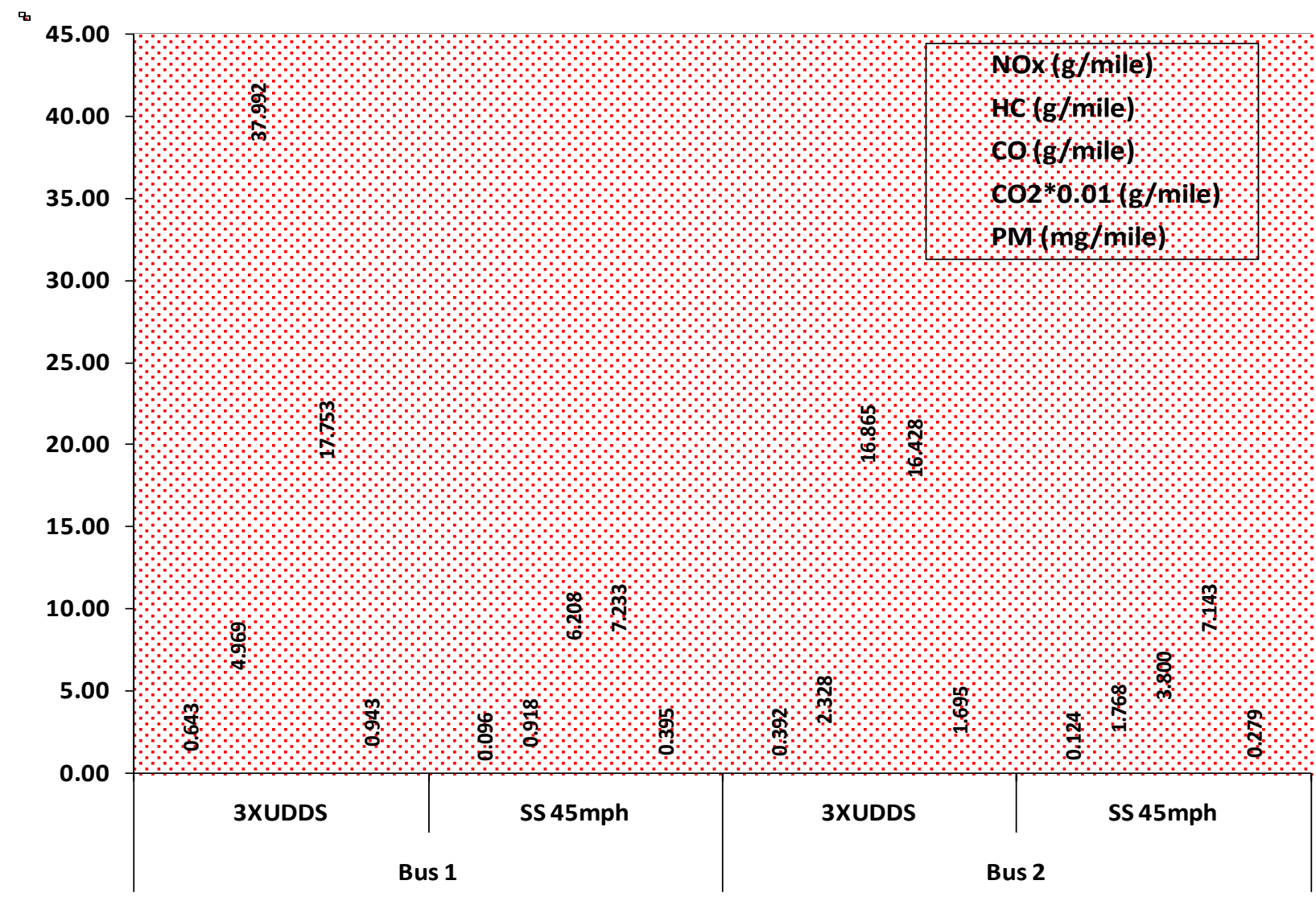

Figure 5-2 Distance specific regulated emissions summary of test vehicles over three different driving cycles.

Oxidation catalysts are more efficient in reducing NMHC than methane. Methane usually requires sustained high exhaust temperatures for complete catalytic light-off. The fact that the Cummins ISLG platform is stoichiometrically fueled, results in sustained high exhaust temperature, even at low load operating conditions. Hence, the THC emissions represented in the figures above, indicate near complete methane emissions with NMHC concentrations below detection limits of a FID analyzer. However, $\mathrm{ppb}$ levels of volatile hydrocarbon could be detected through gas chromatography analysis of canister samples and results of such analysis are presented in section 5.2.3.

\subsection{UNREGULATED EMISSIONS}

The study involved the speciation of carbonyl, PAH compounds, EC/OC and VOC. PAH compounds were extracted and quantified by South West Research Institute (SWRI) and carbonyl, EC/OC and VOC were analyzed by Monitoring Laboratory Divisions (MLD) of CARB. 
Raw data reported by the different institutions were reduced with tunnel flow rates to calculate grams of species in the dilution tunnel.

The results are reported uncorrected for background, with background values plotted separately as tunnel background concentrations. This method has been adopted to avoid reporting negative background corrected test values in cases of concentrations with high measurement uncertainty due to measurement close to or below detection limit.

\subsubsection{POLY AROMATIC HYDROCARBONS (PAH) EMISSIONS}

The PAH data contains speciation results of 22 different PAH compounds. PAH compounds have major health impacts and EPA has classified these pollutants under the Hazardous Air Pollutants (HAP) category. PAH compounds are considered to be carcinogenic in nature. Emissions of PAH compounds are a characteristic to diesel engines, and these compounds are most often associated with PM fraction of diesel emissions. However, their concentrations in CNG exhaust have found to be minimal and mostly originating from lubrication oil combustion. Sampling of PAH compounds was targeted towards three phases, namely gas phase, semivolatile phase and particle phase. The gas phase and semi-volatile phase are captured in the PUF/XAD cartridge and the particle phase is captured in the $90 \mathrm{~mm}$ T60A20 upstream filter. 


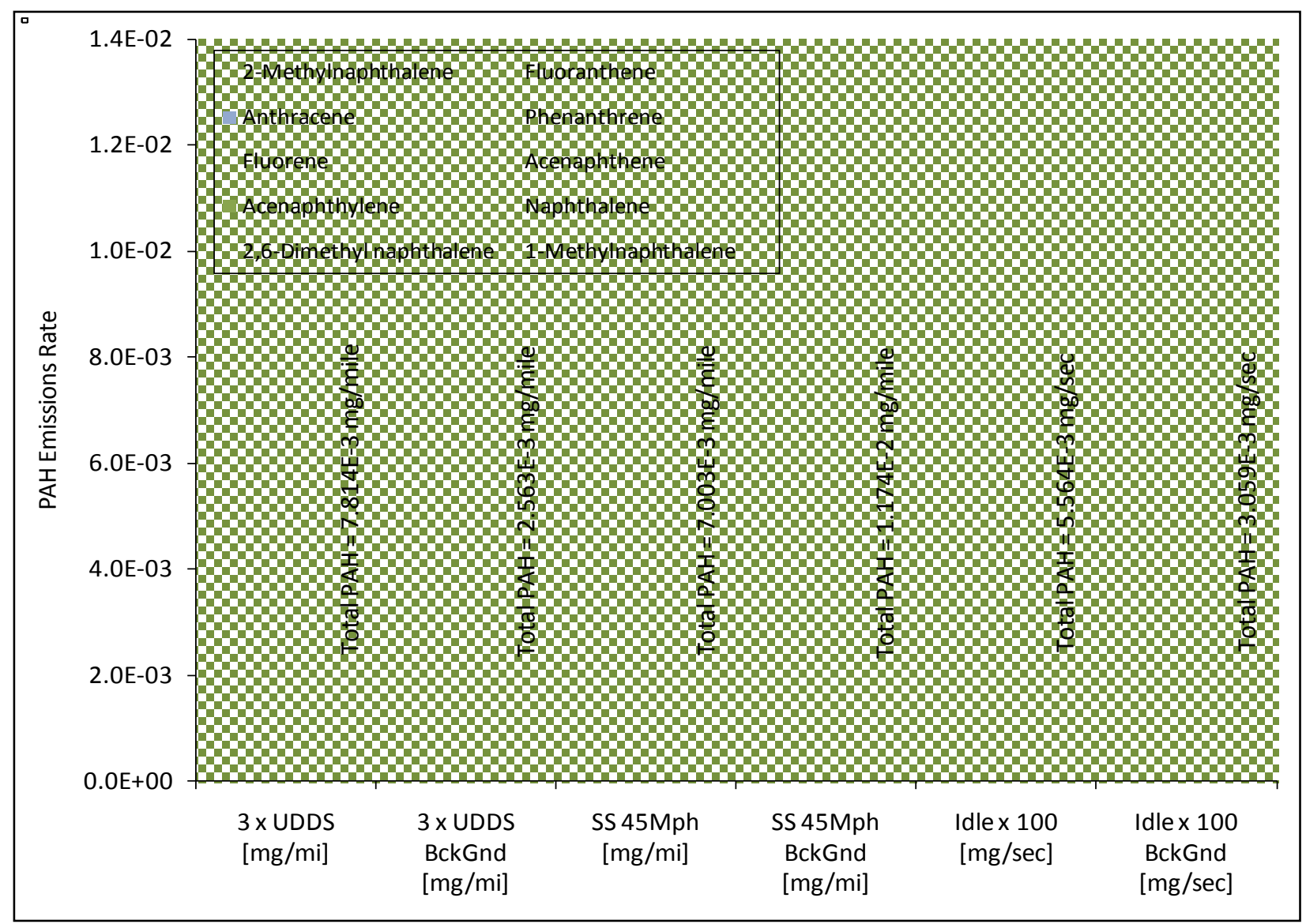

Figure 5-3 Distance specific (time-specific for idle) PAH emissions results of CNG bus 1

Figure 5-3 and Figure 5-4 and represent the distance-specific PAH emissions of bus 1 and bus 2 respectively. Only analytes whose concentrations were found to be within the detection limits of the instruments have been reported. SWRI had assigned a zero concentration for analytes below detection limit.

The PAH emissions from the two buses were observed to be the same order of magnitude. With the absence of aromatic content in the fuel, the source of PAH emissions could be attributed to the lubrication oil consumption in the engine. Naphthalene was found to be the major contributor towards $\mathrm{PAH}$ emissions in both vehicles. Also, significantly high PAH concentrations were detected in the tunnel background samples. Similar observations were documented during in previous studies conducted in California. 


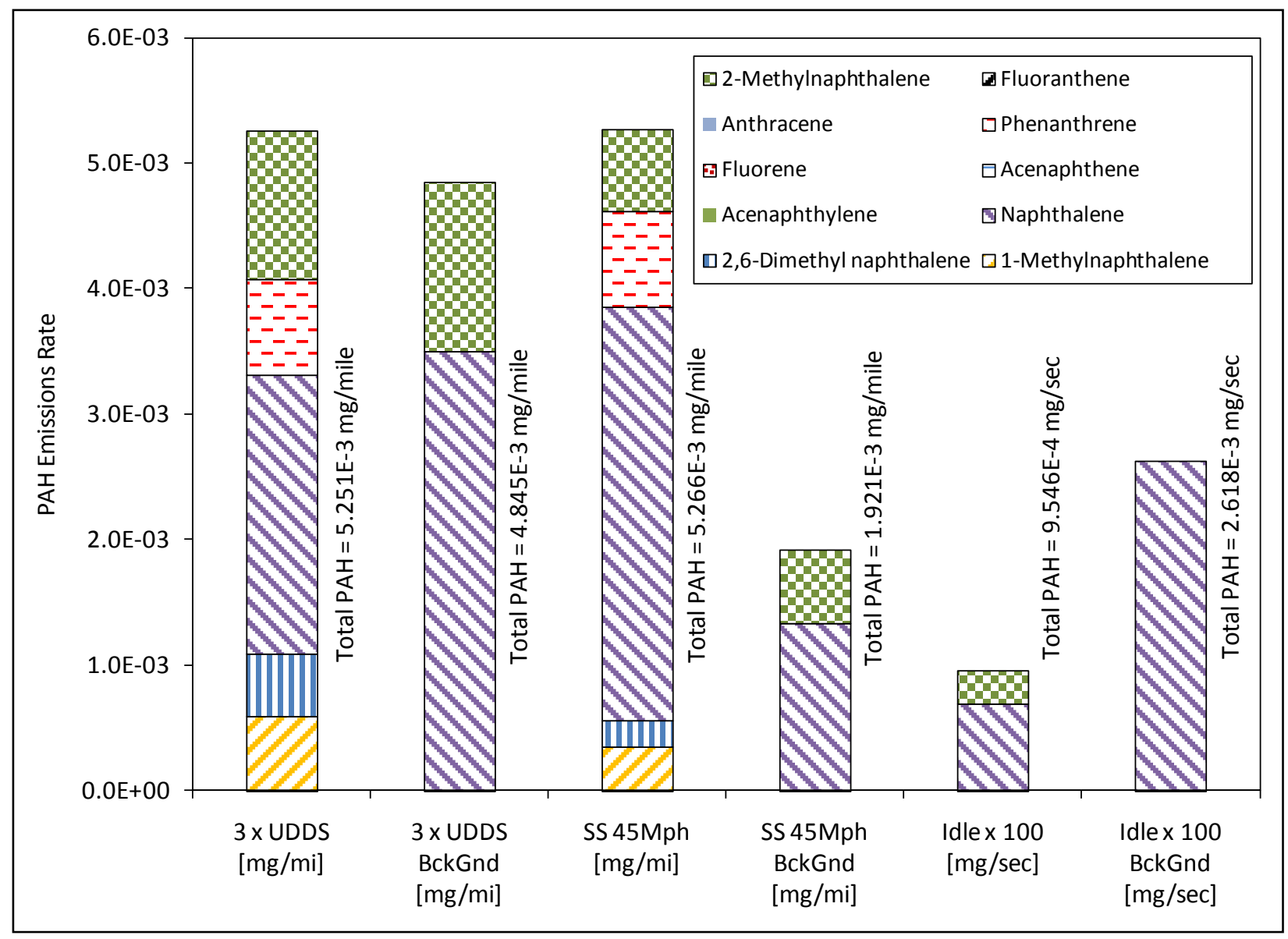

Figure 5-4 Distance specific (time specific for idle) PAH emissions results of CNG bus 2

\subsubsection{ELEMENTAL CARBON/ORGANIC CARBON (EC/OC)}

EC/OC data was reported by CARB MLD. Samples were collected on to pre-fired quartz filters and shipped to MLD for EC/OC quantification. The analysis method involved the ThermalOptical Reflectance (TOR) and Transmittance (TOT) procedure. TOT procedure is based on the EPA/NIOSH 5040 method and reports corerected EC fraction that take into account the pyrolysis of OC during the analysis procedure. The method quantifies 4 different $O C$ fractions depending on the amount of $\mathrm{CO}_{2}$ liberated at different temperatures and oxidizing environments. Also, reported is the EC fraction oxidized above $550^{\circ} \mathrm{C}$. TOR method utilizes a laser and a photo diode to monitor the reflectance of the light non-absorbing $\mathrm{OC}$ and the light absorbing EC, to quantify the pyrolyzed portion of the OC. Similarly, the transmittance method utilizes a laser photo diode and a photocell to detect the transmittance of the filter. Both the 
transmittance and reflectance aid in correcting for the organic fraction that was pyrolyzed and converted to EC during the heat ramps.

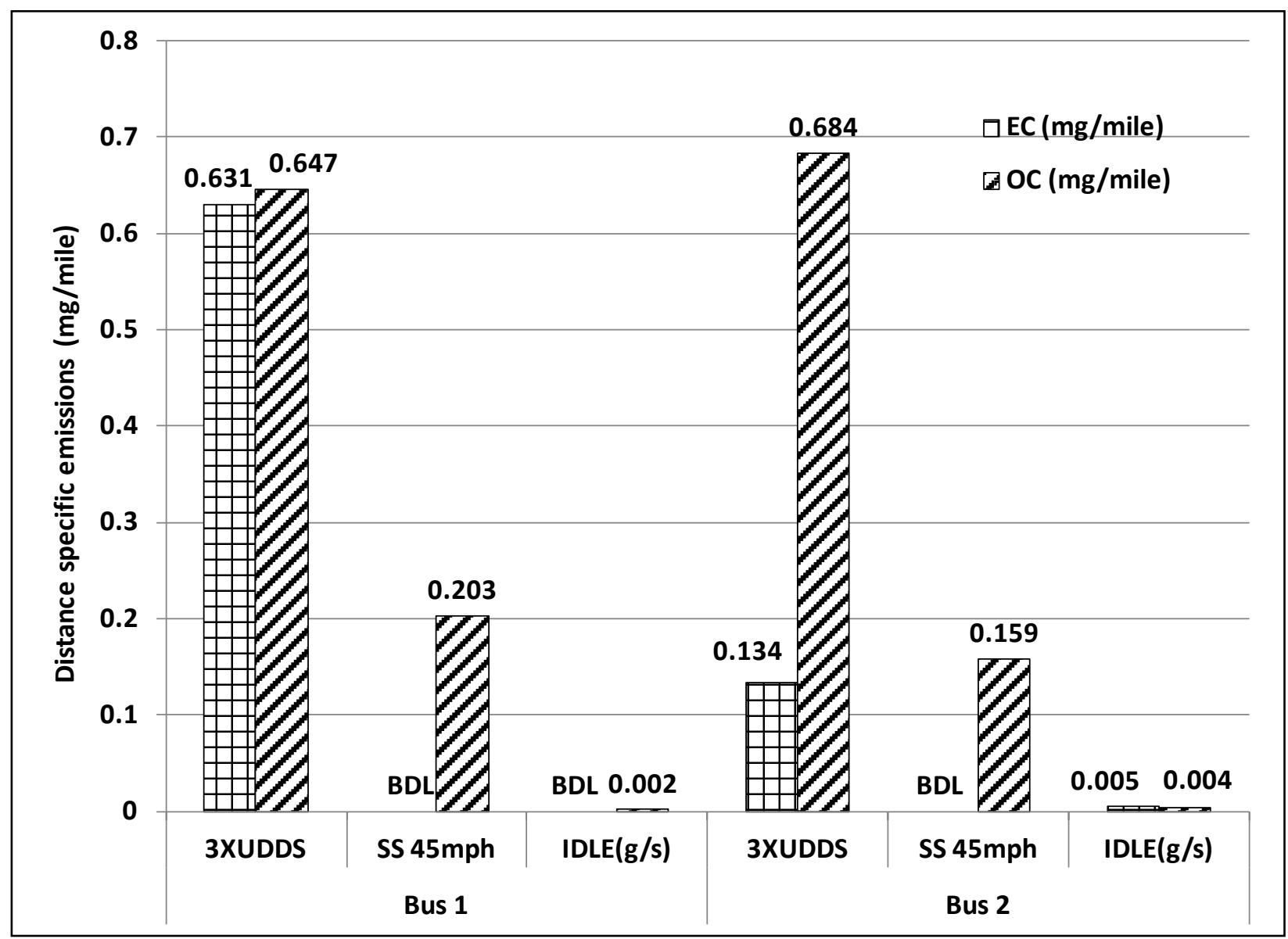

Figure 5-5 Distance specific EC and OC emissions results of bus 1

Figure 5-5 show the distance specific EC and OC emissions for bus1 and bus 2 respectively. Results of all the different OC and EC quantification methods for a given cycle have been plotted. It can be seen that the PM from the CNG buses were completely organic in nature and is consistent with the established theory of CNG vehicles exhaust being completely or very low on EC concentrations. The TEC observed in the UDDS cycle of bus 1could be an outlier in the transmittance data. EC and OC emissions during the idle of bus 1 were observed to be close to background levels.

The organic fraction content of PM from bus 2 is similar to that observed in bus 1 . A significant contribution in EC carbon is also observed and reported by the transmittance and the reflectance method. However, the EC fraction was reported to be below detection limit. 
The source of the EC in these samples could be likely attributed to lubrication oil combustion, during the low speed, and idle modes of the UDDS cycle. Also, to support the argument $0.005 \mathrm{mg} / \mathrm{sec}$ of EC fraction is also observed during the idle mode operation.

\subsubsection{VOLATILE ORGANIC COMPOUNDS (VOC)}

VOC analysis was carried out in cooperation with CARB MLD and data post processing was performed by WVU to report emissions as distance specific VOC emissions. 28 different VOC compounds were quantified. Concentrations reported to be below detection limits have been omitted in Figure 5-6 and Figure 5-7.

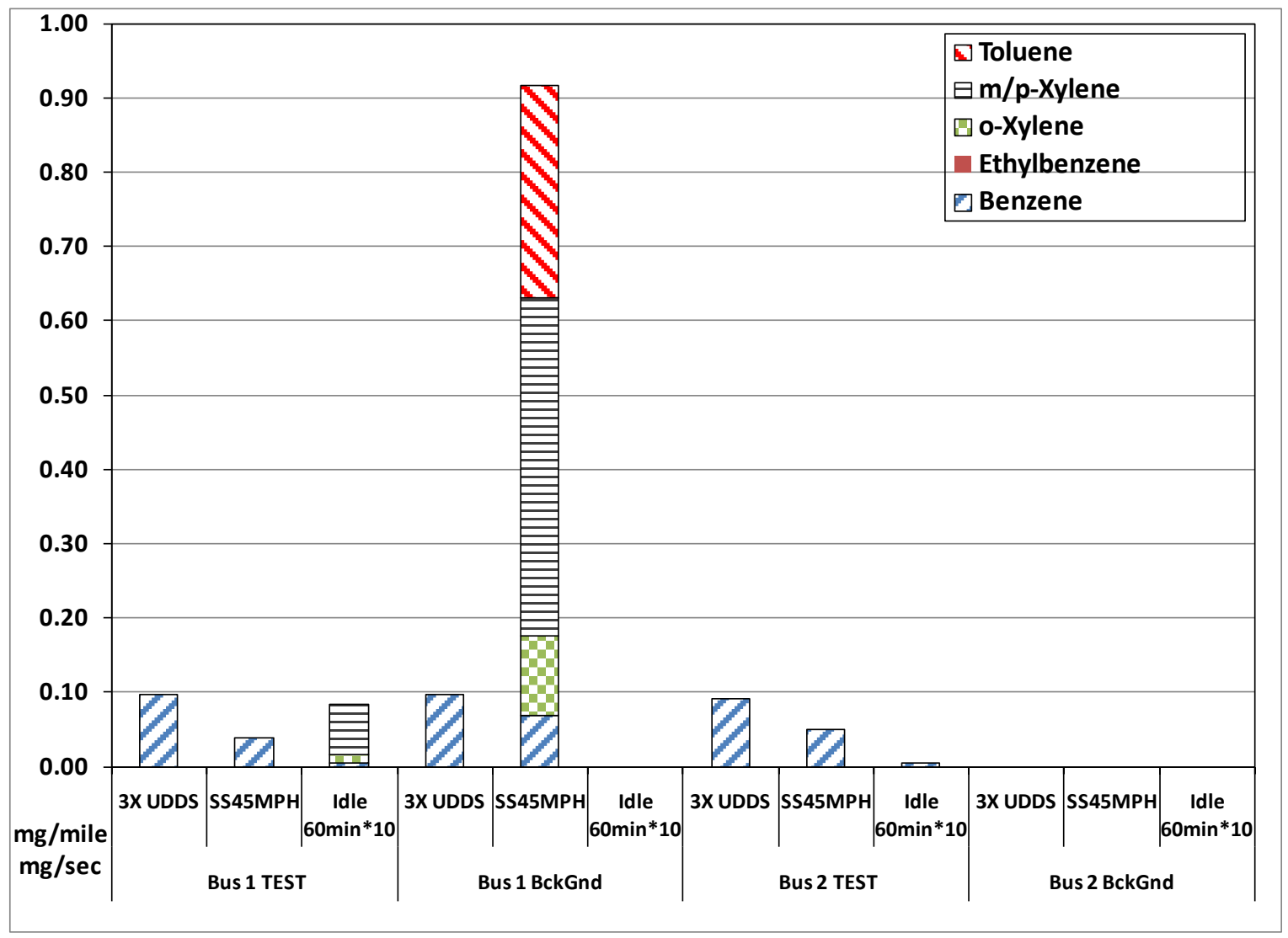

Figure 5-6 Distance specific BTEX emissions results from bus 1 and bus 2

Figure 5-6 shows the comparison of benzene, toluene, ethyl benzene and isomers of xylene (BTEX) emissions from bus 1 and bus 2. BTEX compounds are part of the EPA list of hazardous air pollutants and indentified to be carcinogenic in nature. Mobile sources are identified as the major source of emission. The presence of the TWC after-treatment system in the test vehicle was effective in reducing the concentrations of most BTEX compounds below detection limits. 
Oxidation catalyst are very effective in reducing higher-chain hydrocarbons over a wide temperature range unlike lower chain hydrocarbons which require higher exhaust temperatures for catalytic light-off. Most concentrations of majority of the targeted species were lower or equal to background concentrations. However, high variations in background concentrations were detected based on the time of day.

Figure 5-7 shows the comparison of ethanol, acrolein and acetone emissions between bus 1 and bus 2 . Since all test concentrations are associated with high background values, the actual contribution of the exhaust to the concentrations of these species are very minimal. Also realtively high background variability due to time of data collection, introduces higher uncertainties in the actual vehicle exhaust emissions of these species.

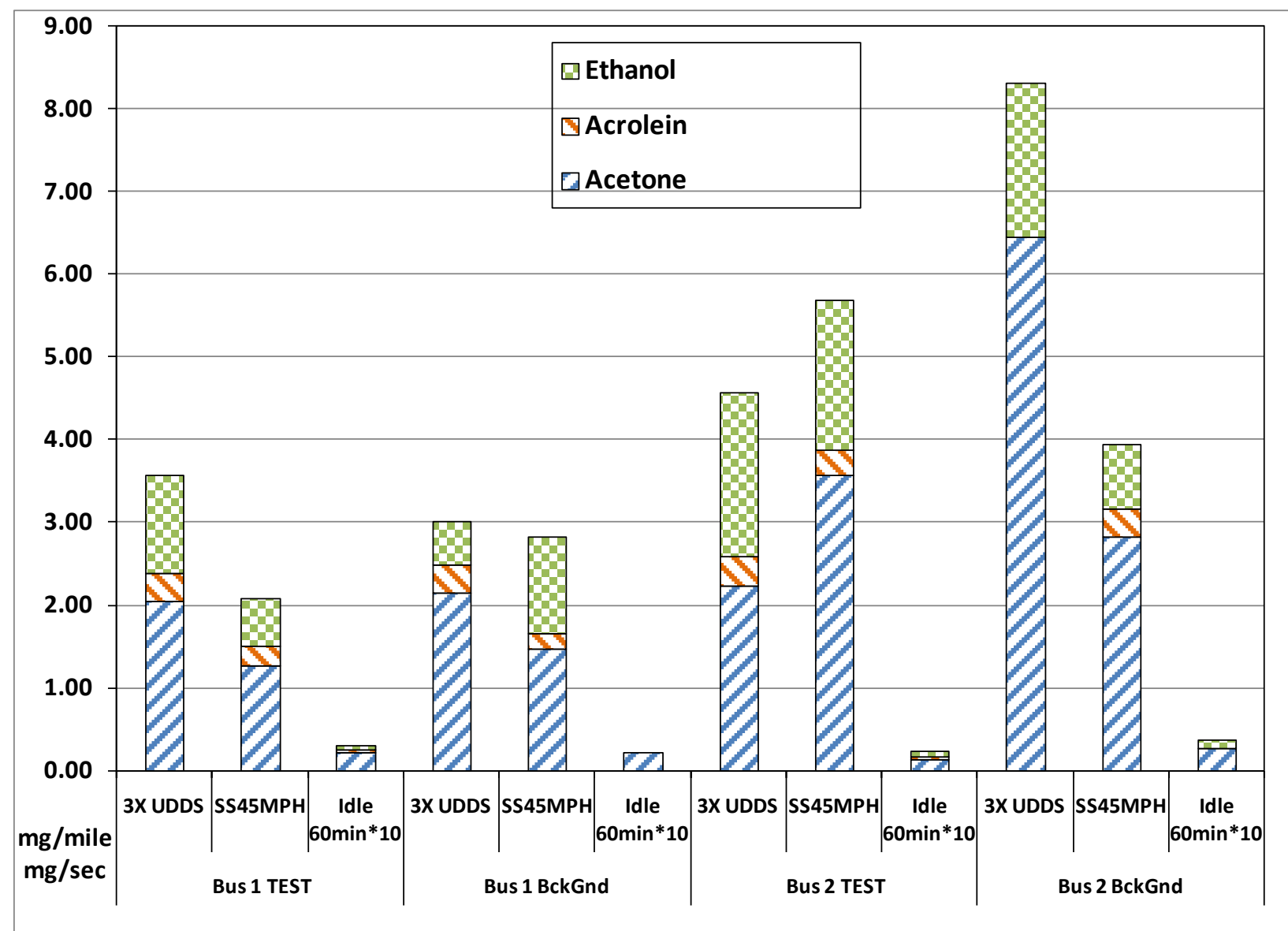

Figure 5-7 Distance specific VOC emissions of bus 1 and bus 2 


\subsubsection{CARBONYL EMISSIONS}

Carbonyl analysis was carried out by CARB MLD. Further data processing was completed by WVU to report distance specific emissions of the carbonyl species. Idle emissions and its background values are represented in units of $\mathrm{mg} / \mathrm{sec}$. Species whose concentration were reported to below detection limits have been omitted from the chart. Figure 5-8 shows the distance specific carbonyl emissions from bus 1 and bus2 over the three test cycles. Formaldehyde and acetaldehyde are commonly observed in significant concentrations in the exhaust of baseline CNG vehicles. However, oxidation catalysts have previously shown to reduce the concentration of most carbonyl species by over $90 \%$. The TWC in the current study has been effective in reducing both formaldehyde and acetaldehyde to levels found in background air. Since the vehicle in the current study was stoichiometrically fueled, exhaust temperatures were sufficient for sustained catalytic activity even in lower engine loads and idle conditions.

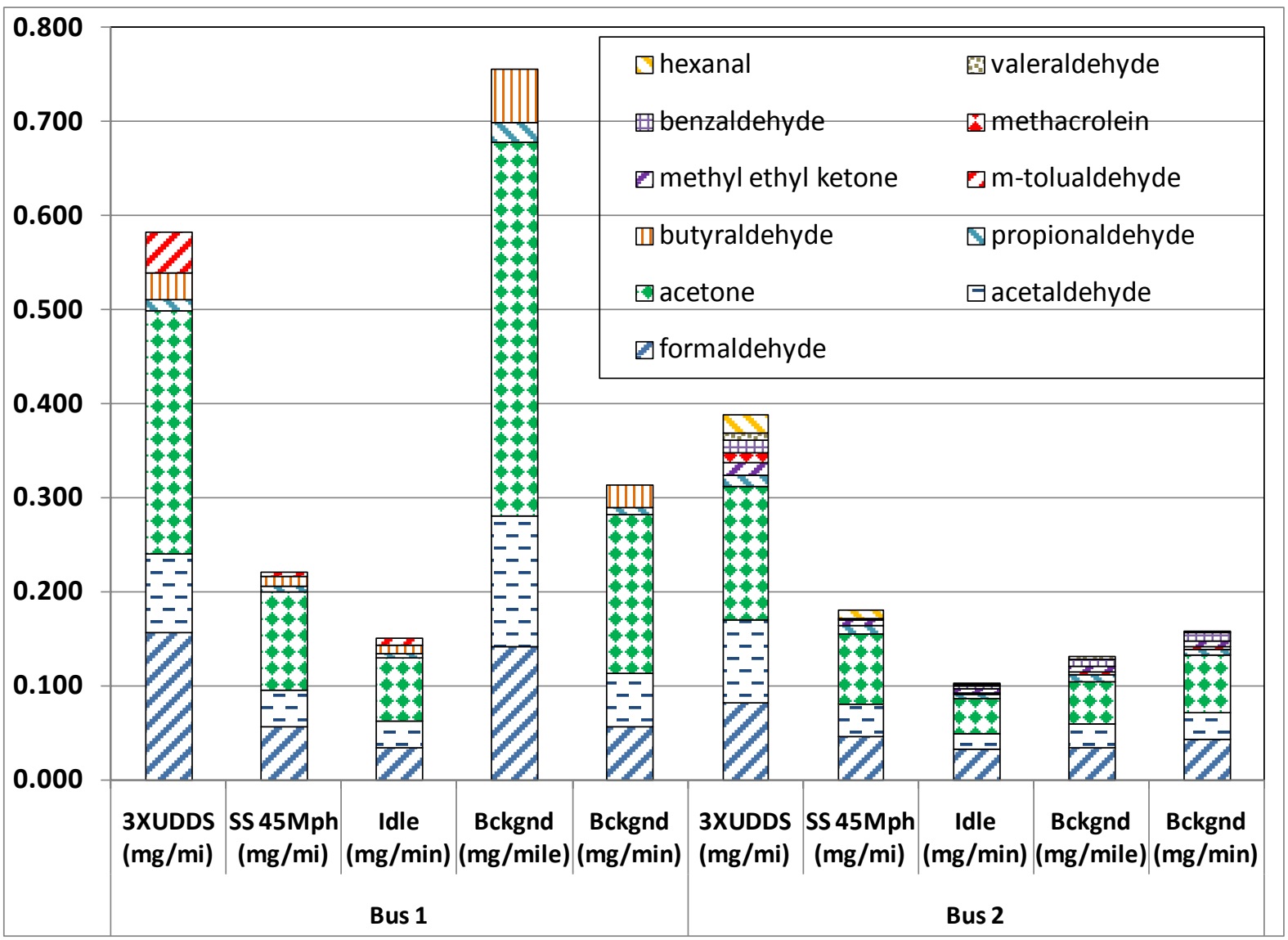

Figure 5-8 Distance specific (time-specific for idle) carbonyl emissions results for CNG bus1 and bus 2 


\subsubsection{WATER SOLUBLE IONS EMISSIONS}

Figure 5-9 show the water soluble ions data for the two CNG buses tested in this study. The CNG fuel being completely devoid of sulfur, did not contribute to any sulfate formation. However, the results do not show a possibility of sulfate formation due to contribution from lubrication oil. All water soluble ions concentrations were close to levels found in the background. Since the concentrations detected were very low, it could be concluded that the water soluble ions results would contain a high level of measurement uncertainty. Significant levels of gas phase ammonia were detected in the exhaust. The ion analysis also further indicates the presence of ammonium in both the test and background samples. The ammonium ions detected in the tunnel blank samples could be tunnel artifacts during the background sample collection period. The ammonium ions in the form of nitrates could have formed during exhaust gas dilution in the CVS system.

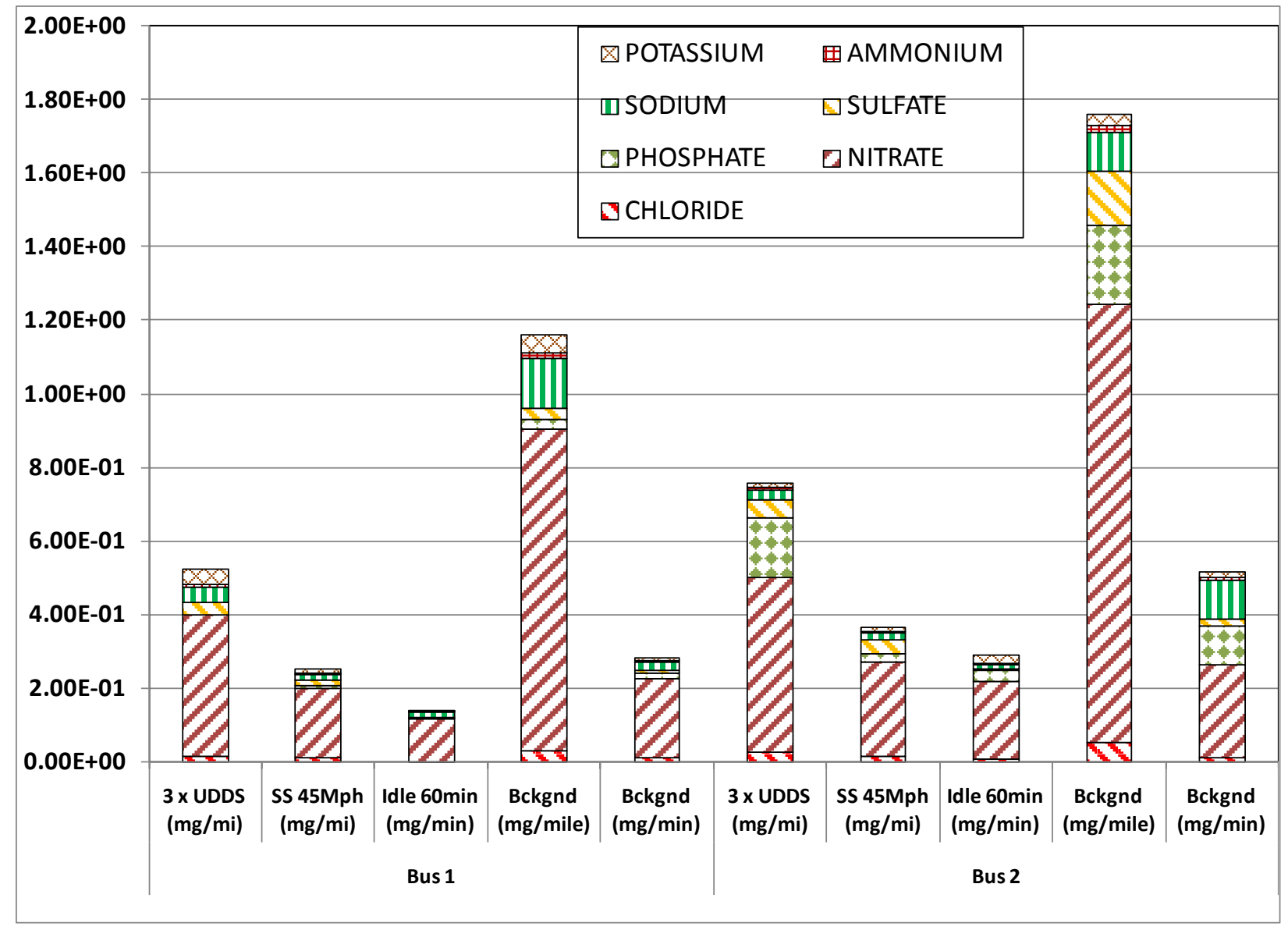

80 | Page 
Figure 5-9 Distance specific (time-specific for idle) water soluble ion emissions results of CNG bus 1 and bus 2

\subsubsection{METALS EMISSIONS}

Figure 24 shows the metals data for the two CNG buses tested in this study. 50 different elements were analyzed through an analysis method involving inductively coupled plasma mass spectroscopy (ICPMS) technique. The chart displays only elements that were detected at levels higher than background concentrations. Calcium were relatively high in the exhaust and background samples. The results of the metal analysis were used to derive correlation between the various toxicity assays, in an attempt to observe the effect of metallic fraction of PM on toxicity. Literature has elucidated the influence of transition metal concentrations towards certain toxicity assays (Verma et al., 2010). Hence, the current study presents correlation of mass fractions of transition metals such as $\mathrm{Cu}, \mathrm{Fe}, \mathrm{V}$ and $\mathrm{Zn}$ with net PM toxicity. Figure 5-10 shows only few of the metals that were detected in significant concentrations in the sample. However, further analysis will present Pearson's correlation for all metallic species to PM toxicity in order to present the dominant metallic species that could possibly contribute to the oxidative potential of engine out PM. 


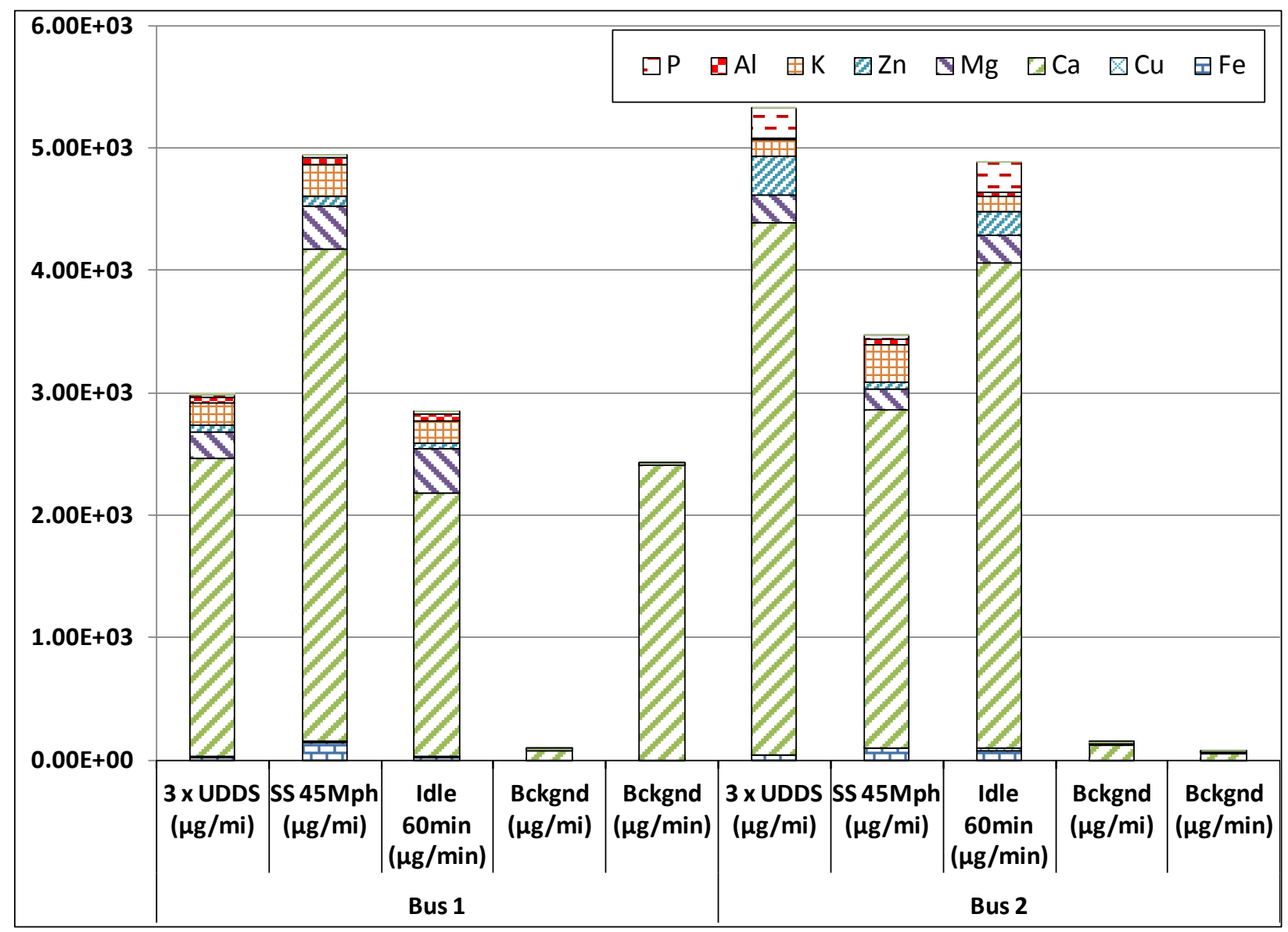

Figure 5-10 Distance specific (time-specific for idle) metals emissions results of CNG bus 1 and bus 2

\subsubsection{OIL ANALYSIS}

Table 9 shows the results of the oil analysis performed on the samples collected from the two vehicles. The oil analysis reported wear metal and additive metals present in the oil sample. The results reported in ppm show calcium, zinc phosphorus as the major additive components present in the oil. Interestingly the oil also shows significant concentration of iron and copper which can be classified as wear metals from engine components. 
Table 9 Results of wear and additive metal analysis in oil samples

\begin{tabular}{cccc}
\hline & $(\mathrm{ppm})$ & Bus 1 & Bus 2 \\
\hline \multirow{4}{*}{ Wear Metals } & $\mathrm{Fe}$ & 19 & 9 \\
& $\mathrm{Cu}$ & 188 & 168 \\
& $\mathrm{Cr}$ & 2 & 2 \\
Additive Metals & $\mathrm{Ca}$ & 1393 & 1391 \\
& $\mathrm{Mg}$ & 54 & 6 \\
& $\mathrm{Zn}$ & 971 & 927 \\
& $\mathrm{P}$ & 640 & 514 \\
Contaminant & $\mathrm{Si}$ & 46 & 4 \\
Metals & $\mathrm{Na}$ & 16 & 16 \\
& $\mathrm{~K}$ & 8 & 1 \\
\hline
\end{tabular}

The result of the oil analysis corroborates the metals and elemental analysis presented in Figure 5-10. $\mathrm{Ca}, \mathrm{P}$ and $\mathrm{Zn}$ are predominant additive metals that are observed both in the oil sample as well as PM samples, hence indicating the direct contribution of lubrication oil to PM.

\subsection{TOXICITY RESULTS}

Three different in-vitro toxicity assays were performed as part of this study. Two cell free assays namely DHBA and DTT assays and one cell based assay using rat alveolar cells known as macrophage ROS assay were performed on the collected PM samples. Both the denuded and the non-denuded PM were extracted from the filter using purified water and further concentrated to be used in each of the assays. The current work will not document the details of the assay procedures, since detailed analysis procedures are documented elsewhere in the literature (Cho et al., 2005, Li et al., 2003, Verma et al., 2010). The toxicity units reported by the three different assays differ by the methodology represented by the assays. The DHBA and DTT assay represent the consumption of a chemical reagent that directly represents electron transfer from oxygen molecule to hydroxyl radicals, which further indicates the onset of oxidative stress or broadly the oxidative potential of a toxic species. Macrophage ROS on the other end depends on the ROS species that are produced within a cell to produce fluorescence in the presence of a certain reagent and the fluorescence produced is measured against a reference control substance called zymosan. As a result the toxicity data is reported as equivalent zymosan units. It is to be noted that, the units accompanying each assay cannot be 
used to conclude the magnitude of the health effect in general. However, the results should be interpreted as an indication to oxidative potential and further correlate the magnitude of the response to magnitude of different PM composition in order to derive conclusions about the various compositions of PM that could drive its overall toxicity. The absolute magnitude of the health effects of PM would be very hard to discern as there exists no correlation or reference between the reported toxicity units and observed health effects. This aspect is one of the main differences between an in-vitro and a in-vivo studies. The results of in-vivo studies indicate the actual end effect of the toxic species in a living animal body.

\subsubsection{DTT ASSAY}

DTT assay was performed by University of California, Los Angeles, School of Medicine. PM samples collected from the two vehicles were placed in analyslides and frozen and shipped over dry ice to the laboratory for analysis. The detailed procedures and experimental setup for the DTT assay is well documented in the works by Cho et al. (Cho et al., 2005) and Li et al.(Li et al., 2003).

Table 10 Results of DTT assay for non-denuded particulate matter

\begin{tabular}{cccccc}
\hline & $\begin{array}{c}\text { Total } \\
\text { Sampling } \\
\text { Time } \\
\text { (hrs) }\end{array}$ & $\begin{array}{c}\text { Total } \\
\text { Distance } \\
\text { (miles) }\end{array}$ & $\begin{array}{c}\text { Total } \\
\text { PM } \\
\text { (mg/mi) }\end{array}$ & $\begin{array}{c}\text { Total PM } \\
\text { (mg/hr) }\end{array}$ & $\begin{array}{c}\text { Non- } \\
\text { Denuded PM } \\
\text { DTT } \\
\text { consumption } \\
\text { (nmole/min) }\end{array}$ \\
\hline $\begin{array}{c}\text { 3X UDDS-Bus 1 } \\
\text { 45 MPH (Bus } \\
\begin{array}{c}\text { 1+Bus 2) } \\
\text { Idle (Bus 1+Bus }\end{array}\end{array}$ & 6.2 & 115.8 & 1.48 & 27.7 & 1885.22 \\
$\begin{array}{c}\text { 2) } \\
\text { 3X UDDS-Bus 2 }\end{array}$ & 19.4 & 1009.5 & 0.17 & 7.65 & 2402.61 \\
\hline
\end{tabular}

Table 10 above shows the results of the DTT assay for the non-denuded PM samples collected from the two buses. It can be seen that the total sample time over each cycle has proceeded over several hours in order to collect sufficient sample for observing responses through the assays. The DTT assay required a minimum of $1 \mathrm{mg}$ of net PM sample collected over the filter for an effective toxicity analysis. Although, extended sampling times and high volume rate sampling rates were employed, the mass emissions rates of PM from most vehicle 
operation specifically during idle and cruise mode operation was very low. Hence, the analysis institution decided to integrate filter samples from both the buses for the $45 \mathrm{MPH}$ and idle operation as one single test data. The process of integrating two filters from different buses poses different challenges during data interpretation. Mainly the differences in mass emission rates of PM between the two buses is averaged to one single data point, and moreover an attempt to produce correlation between toxicity and various PM fractions becomes less accurate due to the availability of lesser data points. However, an analysis carried out with insufficient mass could have most likely resulted in toxicity levels below detection limits no discernible data to study the composition of PM that would drive the oxidative stress reactions. The process of integrating filter samples resulted in observable toxicity responses from the DTT assay.

The DTT assay uses the consumption of a chemical called dithiothreitol (DTT) as a marker for quantifying oxidative stress. Hence the DTT activity unit "nmole/min" represents the consumption rate of DTT in the presence of the PM sample.

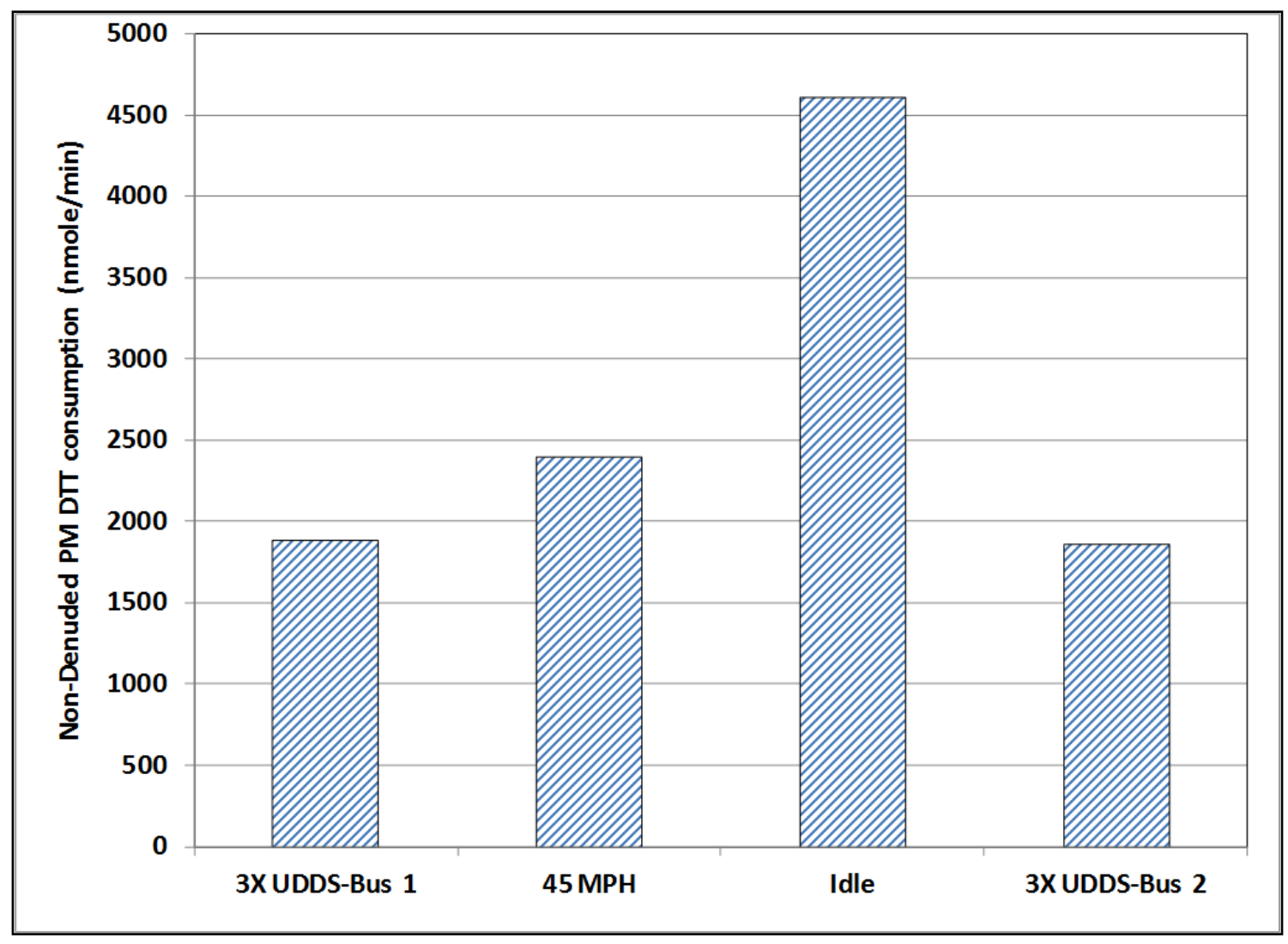


Figure 5-11 Results of DTT consumption rate for different driving cycles

Figure 5-11 shows the bar chart of the total PM mass specific DTT activity of the nondenuded PM sample. Since the steady state and idle operation did not result in adequate PM loading, samples from the two buses were integrated into one. Hence, the values represented here can be viewed as an average toxicity of two vehicles over the same driving cycle. It is to be noted that although the PM mass emissions from the steady state operation did vary significantly, PM mass emission rate during idle from bus 2 was an order of magnitude higher than Bus 1.

Figure 5-12 shows the mass specific DTT activity over the different driving cycles. The results show mass specific DTT activity to be of the same order of magnitude over all driving cycles. The order of magnitude higher mass specific DTT activity from bus 2 during UDDS cycle is a result of normalizing measured DTT activity to a lower PM mass collected on the filter samples over this cycle.

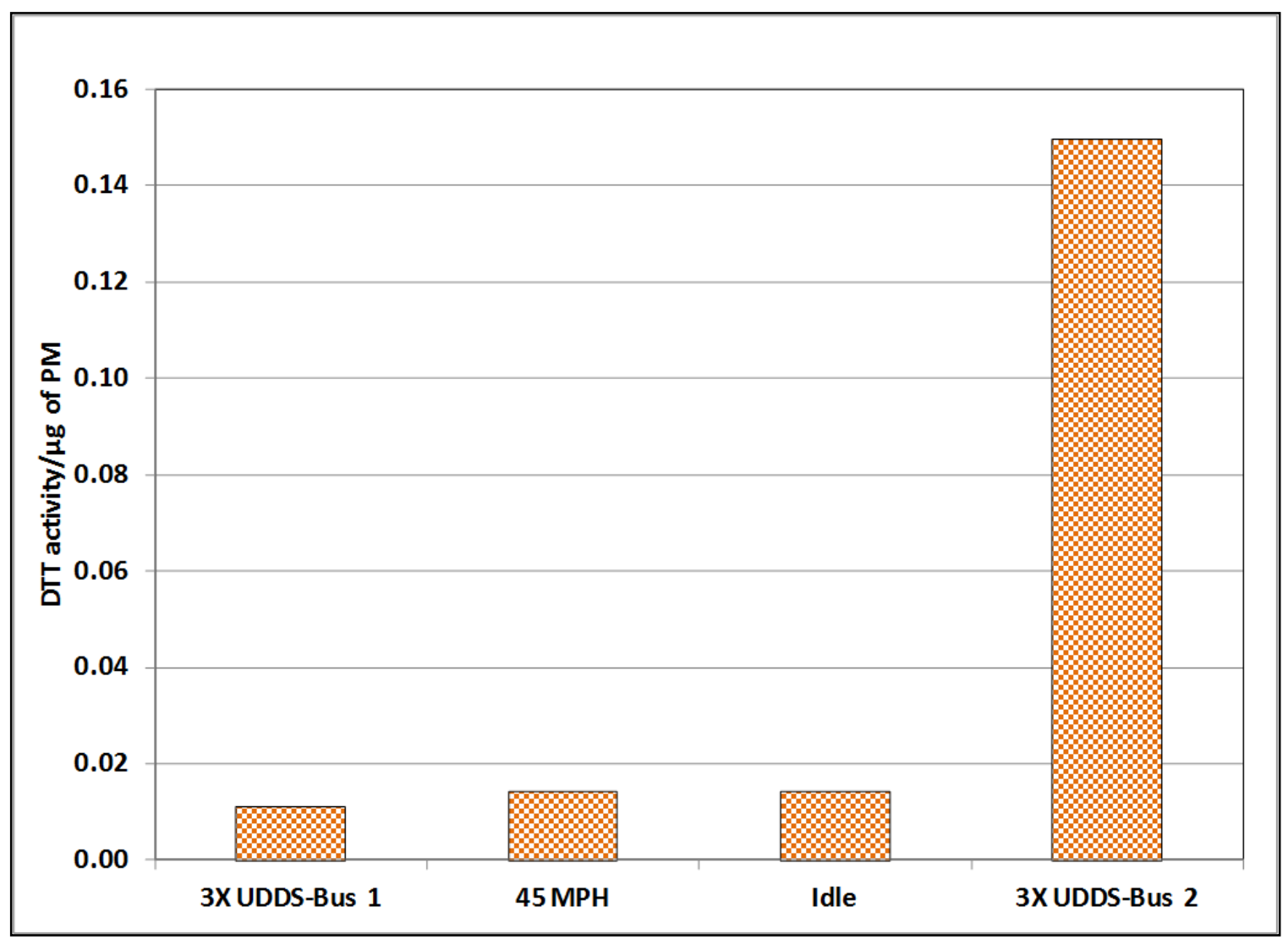

Figure 5-12 PM mass specific DTT activity over driving cycles 


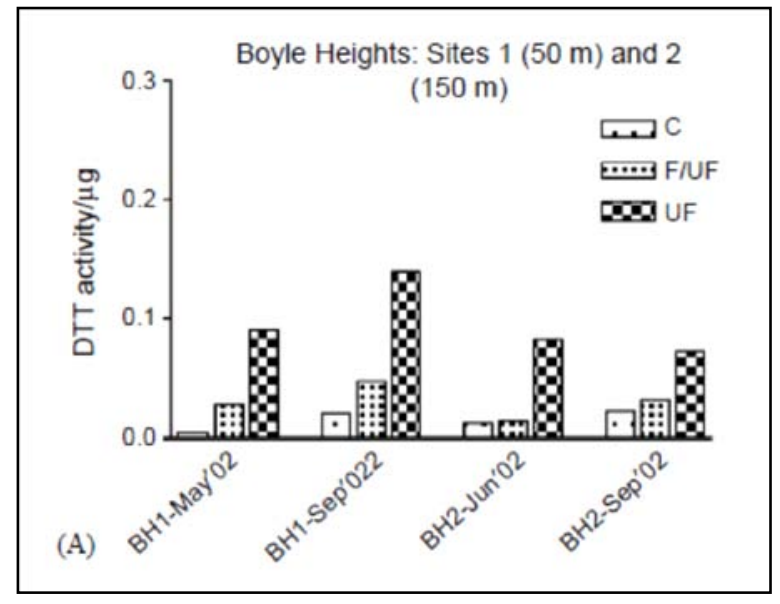

Figure 5-13 Ambient DTT activity levels in Boyle Heights, CA (Cho et al., 2005)

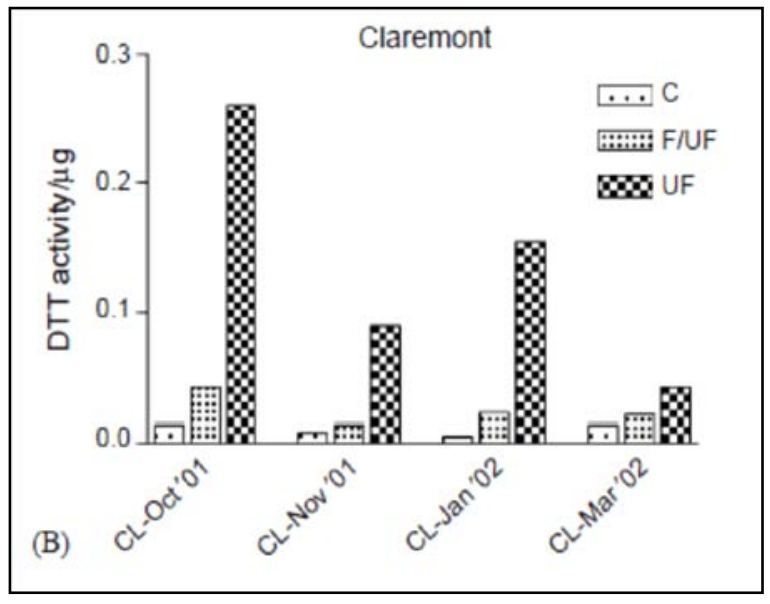

Figure 5-14 Ambient DTT activity levels in Calremont, CA (Cho et al., 2005)

Figure 5-13 and Figure 5-14 serve as a reference to compare the results obtained in this study to previously observed ambient DTT activity in certain regions of California (Cho et al., 2005). It can be seen that DTT activity of heavy-duty natural gas vehicle PM samples is on an average an order of magnitude lesser than DTT activity of ambient PM. 


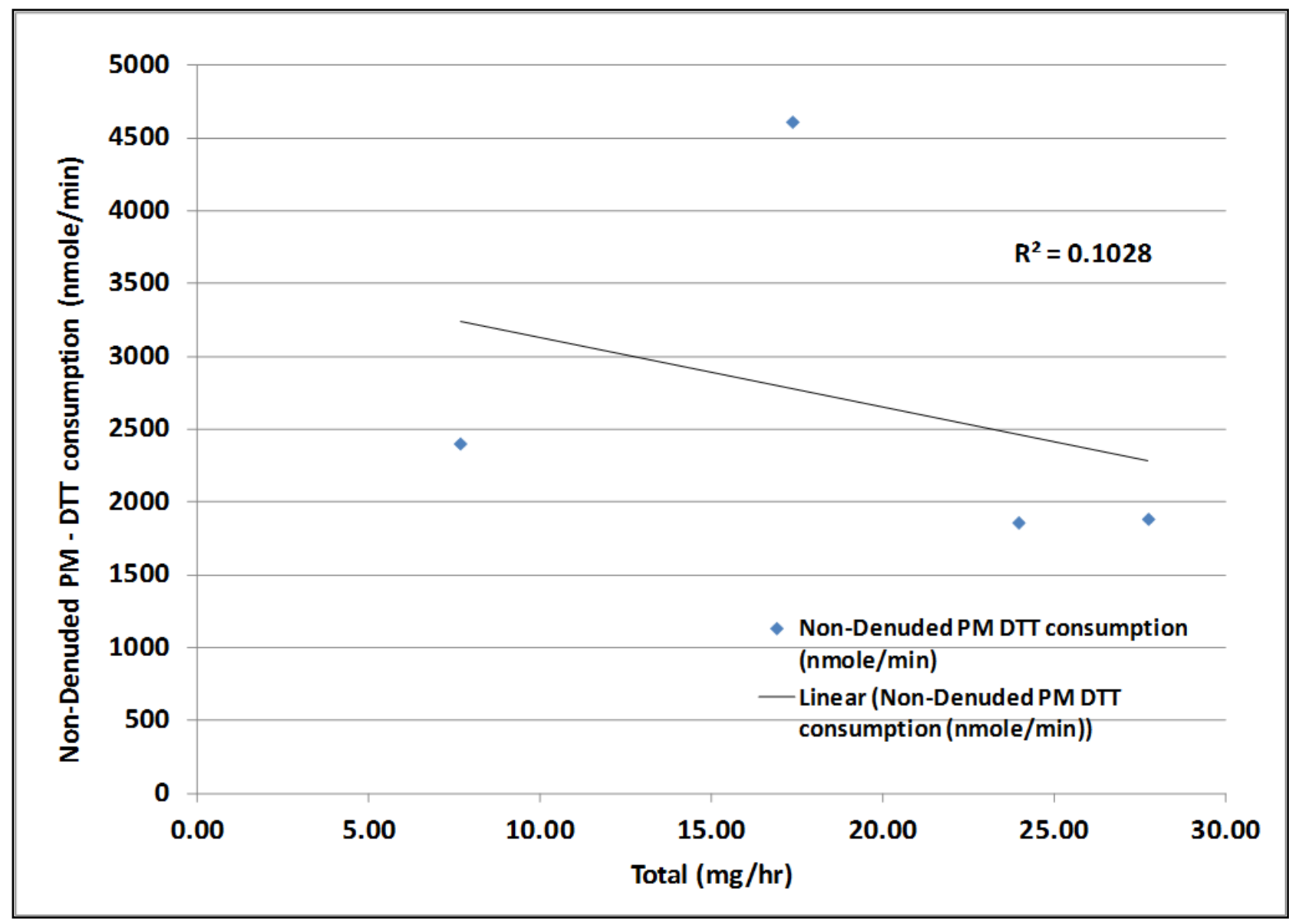

Figure 5-15 Scatter plot of total PM emission rates over different driving cycle vs. DTT consumption rate

Figure 5-15 shows the scatter plot between DTT consumption rate of non-denuded particulate matter and mass emissions rate of PM over different driving cycles. It can be seen that no significant correlation exists between DTT activity and total mass emissions rate of PM. The results show that total mass emissions of PM, is not directly related to DTT consumption rate. Hence, detailed compositional analysis must be presented to illustrate the dependence of DTT activity on individual fractions that constitute the total PM emissions.

\subsubsection{ELEMENTAL COMPOSITION VS. DTT ACTIVITY}

Literatures reviewed in section 2.4.1.1 have documented the influence of transition metals specifically copper and iron in increased DTT activity. This section will provide correlation analysis of various metals that contributed to significant mass emissions from the heavy-duty 
natural gas vehicle. The metals analyzed include transition metals and metals that are markers for lubrication oil influence such as S, P and Zn.

Table 11 Pearson Correlation coefficient (R) and level of significance (p) for elemental species Vs. DTT activity

\begin{tabular}{ccc}
\hline Species & $\mathrm{R}$ & $\mathrm{p}$ \\
\hline $\mathrm{Cu}$ & $\mathbf{0 . 9 9}$ & $\mathbf{0 . 0 1}$ \\
$\mathrm{Mg}$ & 0.84 & 0.16 \\
$\mathrm{Al}$ & 0.79 & 0.21 \\
$\mathbf{P}$ & $\mathbf{0 . 9 9}$ & $\mathbf{0 . 0 1}$ \\
$\mathrm{S}$ & 0.57 & 0.43 \\
$\mathrm{~K}$ & 0.43 & 0.57 \\
$\mathrm{Ca}$ & 0.73 & 0.26 \\
$\mathrm{~V}$ & 0.57 & 0.43 \\
$\mathrm{Cr}$ & 0.81 & 0.19 \\
$\mathrm{Mn}$ & 0.70 & 0.30 \\
$\mathrm{Fe}$ & 0.28 & 0.72 \\
$\mathrm{Co}$ & -0.24 & 0.76 \\
Zn $^{*}$ & $\mathbf{0 . 9 3}^{*}$ & $\mathbf{0 . 0 7}$ \\
\hline
\end{tabular}

Table 11 shows the Pearson correlation between total mass of elements measured and measured DTT activity. Results show that $\mathrm{Cu}(\mathrm{R}=0.99)$ and $P(R=0.98)$ exhibits a significant correlation $(p<0.05)$ towards DTT activity. For the current data set, the R value in the $95 \%$ confidence interval is 0.95 and in the $90 \%$ confidence interval is 0.90 . Both $\mathrm{Cu}$ and $\mathrm{P}$ show significant correlation in the 95\% confidence interval whereas $\mathrm{Zn}(\mathrm{R}=0.93)$ falls within the $90 \%$ confidence interval. Hence, in further analysis $\mathrm{Zn}$ will also be considered as a significant factor influencing DTT activity of PM samples. The results shown here further corroborate the findings of other studies that have illustrated the potency of transition metals to induce oxidative stress. One of the surprising findings of the current study is the low correlation of DTT activity to Fe mass content in PM. The lower correlation of Fe in comparison to $\mathrm{Cu}$ could be due to the fact that the DTT activity is extremely sensitive to $\mathrm{Cu}$ and Mn (DTT consumption is approximately 300 times faster) in comparison to Fe (Charrier and Anastasio, 2012). Hence as a result we could be observing the masking effect of copper over the possible DTT activity of Fe. 
It is to be noted that the presence of Cu can be viewed as a sampling artifact, as the use of $\mathrm{Cu}$ in exhaust after-treatment systems has been prohibited by the US regulatory agencies. Although, the influence of copper on DTT activity is pronounced, the contribution of $\mathrm{Zn}$ and P towards the DTT activity suggests the role of lubrication oil emissions in PM toxicity. A report presented by Desert Research Institute (DRI) documents that Zn, P and S are the dominant elements' found in lubrication oil that manifests itself in exhaust PM emissions also (Fujita et al., 2006).

Metal chelation is a process through which the oxidative effect of metals can be suppressed by either addition of a chemical reagent or through removal of metals from the sample. In the case of the DTT assay a reagent called DTPA (Diethylenetriamine pentaacetic acid) is used to suppress the activity of transition metals to observe the possible effect of other species towards DTT consumption rate. The results showed no DTT consumption rate post metal chelation process, indicating a complete contribution of transition metals $\mathrm{Cu}$ and $\mathrm{Zn}$ and possibly the presence of non-metal phosphorus towards DTT consumption.

DTT activity of the thermally denuded PM was of the same of order of magnitude of the non-denuded PM, further supporting the argument of the contribution elements and metals to the observed DTT activity. Since the denuded PM mass was insufficient to generate toxicity data individually for bus1 and bus 2, filter samples from both buses were integrated as one single sample set. As a result, the toxicity data from the denuded PM sample could only be used as an evidence to support the correlation obtained through the non-denuded PM samples. Since, the process of thermal denuding removes only the volatile species, the effect of metals on the toxicity of the denuded PM sample should theoretically remain the same.

\subsubsection{PAH, ELEMENTAL CARBON AND ORGANIC FRACTION VS. DTT ACTIVITY}

The analysis in this section presents correlation between PAH compounds, elemental carbon and organic carbon mass to DTT activity. Most PAH compounds were found to be below detection limits. However, a few lighter PAH compounds (molecular weight less than 200) were observed to be significantly higher background concentrations. Hence, the total PAH emissions for compounds with molecular weight less than 200 were chosen to be correlated with DTT 
activity. DTT activity has shown to be sensitive towards OC concentrations, however, OC analysis from pre-fired quartz filters, in the case of natural gas vehicle exhaust could be biased with large positive sampling artifact and hence appear to not correlate well with DTT activity. This could be mainly attributed to the fact that the OC measured could be simply gas phase lower chain hydrocarbons (predominantly methane) adsorbed onto the filter rater than particle phase organic carbon formed as result of gas to particle conversion.

Table 12 Pearson correlation for volatile, semi-volatile and elemental carbon compounds Vs. DTT activity

\begin{tabular}{ccc}
\hline Species & $\mathrm{R}$ & $\mathrm{p}$ \\
\hline PAH & & \\
(MW<200) & $\mathbf{0 . 9 8}$ & $\mathbf{0 . 0 1}$ \\
OC & 0.78 & 0.21 \\
EC & 0.84 & 0.16 \\
\hline
\end{tabular}

Table 12 shows the Pearson correlation for PAH compounds with molecular weight less than 200, organic carbon and elemental carbon emissions to the measured DTT activity of the non-denuded PM sample. Only PAH compounds show statistically significant $(p<0.05)$ correlation with DTT activity. The results obtained in this study is contrary to results reported in the work by Ntziachristos et al. which showed low correlation coefficients for PAH compounds with molecular weight less than 250 (Ntziachristos et al., 2007). However, it is to be noted that the cited literature is based on ambient PM, and it differs significantly in the type of PAH compounds detected in the sample. PAH compounds that were observed to be in significant concentrations in the current study are characteristic of natural gas vehicle emissions. The absence of aromatic content in fuel results in minimal higher chain PAH compounds concentration and is characterized by lower chain PAH compounds such as phenanthrene, and isomers of methyl naphtlene. Although, no direct particle formation mechanisms have been documented by these compounds, it is possible that lower chain PAH compounds can readily adsorb onto other particle sites such as metal species and contribute to particle phase PM. The high correlation coefficient for the light PAH compounds suggest a possible combined redox activity of transition metals and PAH compounds. 
Organic carbon and elemental carbon showed no statistical significant correlation with DTT activity. This can be further substantiated with the results of the post metal chelation DTT activity suggesting no redox activity from any other fraction of PM other than metals.

\subsubsection{MULTIVARIATE REGRESSION FOR DTT ACTIVITY}

Since more than one factor presented statistically good correlation with DTT activity, a multivariate regression was performed to establish a relationship between the different factors in predicting DTT activity. Matlab statistical tool was used to perform the multivariate regressions analysis. The general equation for multivariate regression is show in Equation 20.

$$
\text { Predicted DTT activity }(Y)=\beta_{0}+\beta_{1} * X_{1}+\beta_{2} * X_{2}+\beta_{3} * X_{3}
$$

Equation 20

Where $\beta_{0}, \beta_{1}, \beta_{2}, \beta_{3}$ are regression coefficients obtained through the analysis.

Two regression analyses were performed by considering the effect of copper and by eliminating the influence of $\mathrm{Cu}$ in DTT activity. The regression analysis as result of the absence of $\mathrm{Cu}$ will essentially indicate the complete contribution of lubrication oil based emissions on DTT activity.

Table 13 lists the results of the two regression analysis performed on the DTT activity data. The coefficients derived from the analysis can be used to predict DTT activity with known masses of the factors considered. 
Table 13 Results of multivariate regression analysis

\begin{tabular}{ccccc}
\hline & Factor & Coefficient & Value & $\mathbf{R}^{\mathbf{2}}$ \\
\hline & Constant & $\beta_{0}$ & 0 & \\
Regression & $\mathrm{Cu}$ & $\beta_{1}$ & 2.681 & \\
$\mathbf{1}$ & $\mathrm{Zn}$ & $\beta_{2}$ & -0.3573 & 1 \\
& $\mathrm{P}$ & $\beta_{3}$ & 0.444 & \\
& $\mathrm{PAH}$ & & & \\
& $(\mathrm{MW}<200)$ & $\beta_{4}$ & -835.4 & \\
Regression & $\mathrm{Zn}$ & $\beta_{\mathrm{O}}$ & 1849.3 & \\
$\mathbf{2}$ & $\mathrm{P}$ & $\beta_{1}$ & 0 & \\
& $\mathrm{PAH}$ & $\beta_{2}$ & 0 & 0.97 \\
& $(\mathrm{MW}<200)$ & $\beta_{3}$ & 0.0235 & \\
\hline
\end{tabular}

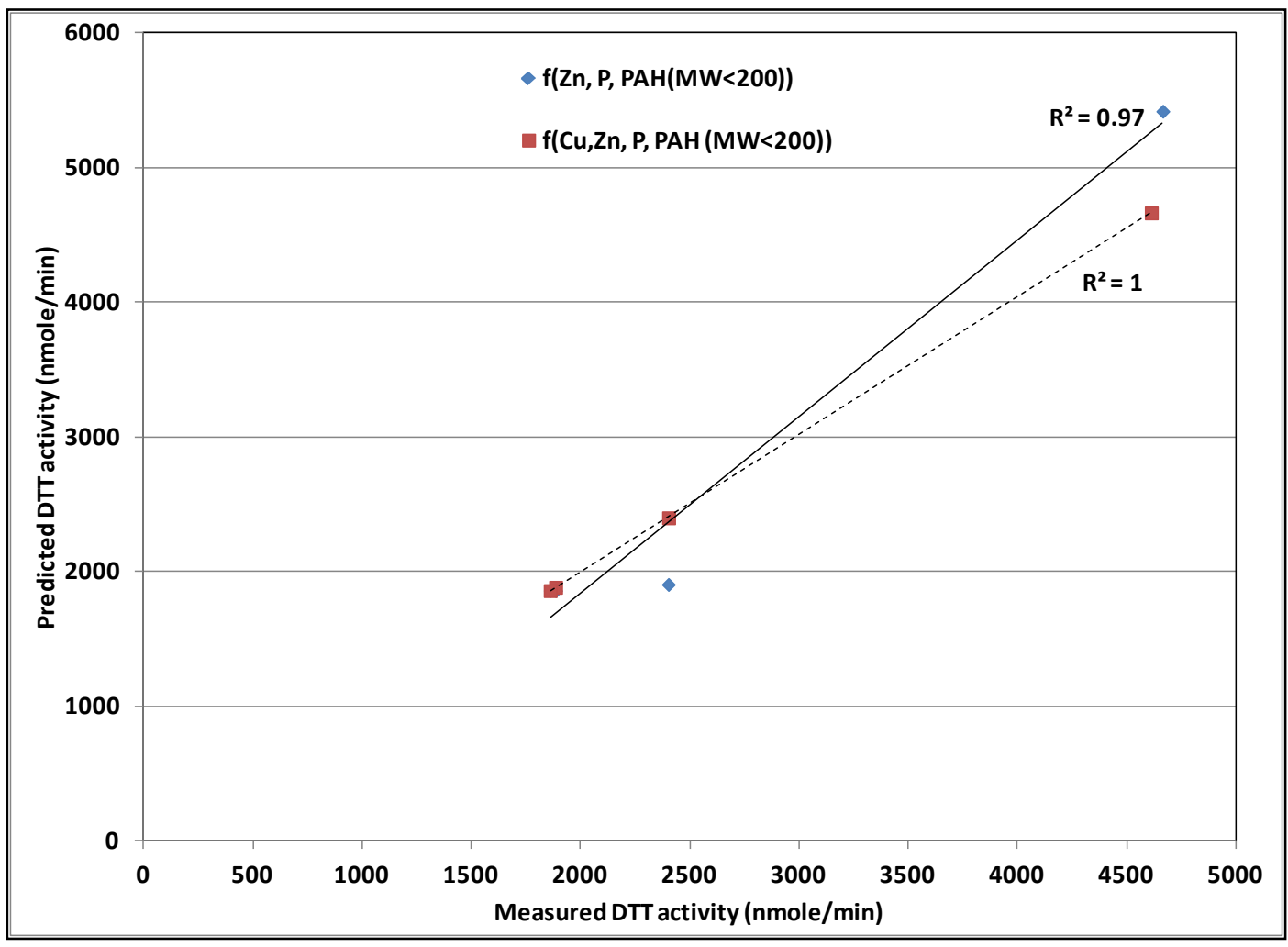

Figure 5-16 Scatter plot of the predicted DTT activity Vs. measured DTT activity 
Figure 5-16 shows the scatter plot between predicted DTT activities as result of the regression analysis to the measured DTT activity. It can be seen that with the inclusion of $\mathrm{Cu}$ as a factor the analysis results in $a R^{2}=1$ and with the exclusion of $C u$ it decreases to $R^{2}=0.97$.

\subsubsection{DHBA ACTIVITY}

The DHBA activity contrary to the DTT activity measures the formation of dihydroxybenzoic acid produced as result of fenton reactions of transition metals with hydroxyl ions. The formation of DHBA can be considered as a marker for the process of consumption of antioxidants within livings cells in the event of exposure to metal concentrations.

The DHBA assay has been a relatively new development in the field of toxicity assays and is targeted towards discerning the effect of metal concentration on cellular oxidative stress. The analysis for the DHBA assay was performed on the same PM extracts used for the DTT analysis. Table 14 shows the results of the DHBA formation rates in nmole/min for the non-denuded PM samples.

Although the same filter extracts were used for the DTT and DHBA assay, it appears that the DTT activity is more sensitive to lower concentrations of PM in comparison to the DHBA assay. The results of the DHBA assay indicate DHBA formation of test samples to be comparable to rates obtained from background filter samples. The results suggest that within the scope of this study, the PM mass emissions rates from heavy-duty natural gas vehicles to be insufficient to exhibit discernible DHBA formation rates in order to provide further analysis. Since, the DTT activity as discussed in the previous section provides insight into the role of PAH and metals present in the same sample, it further supports my assumption of the lower sensitivity of the DHBA assay towards ultra-low PM emission rates. 
Table 14 Results for DHBA formation of non-denuded particulate matter.

\begin{tabular}{cccccc}
\hline & $\begin{array}{c}\text { Total } \\
\text { Sampling }\end{array}$ & $\begin{array}{c}\text { Total } \\
\text { Distance } \\
\text { (miles) }\end{array}$ & $\begin{array}{c}\text { Total } \\
\text { PM } \\
\text { (mg/mi) }\end{array}$ & $\begin{array}{c}\text { Total PM } \\
\text { (mg/hr) }\end{array}$ & $\begin{array}{c}\text { Non- } \\
\text { Denuded PM } \\
\text { DHBA } \\
\text { formation } \\
\text { (nmole/min) }\end{array}$ \\
\hline 3X UDDS-Bus 1 & 6.2 & 115.8 & 1.48 & 27.72 & 599.84 \\
45 MPH & 22.4 & 1009.5 & 0.17 & 7.65 & $1716.15^{*}$ \\
Idle & 19.0 & NA & NA & 17.34 & 527.14 \\
3X UDDS-Bus 2 & 9.7 & 181.7 & 1.28 & 23.94 & 558.18 \\
\hline
\end{tabular}

* $45 \mathrm{MPH}$ test was characterized by very low PM mass and as a result the DHBA formation rate for the test was characterized by high measurement uncertainty and values lower than background PM samples DHBA formation rate.

Table 15 presents the Pearson correlation coefficient for elemental species with observed DHBA formation rate. It can be seen that no statistical significance in the $95 \%$ confidence interval exists $(p<0.05)$ for any of the metals or elemental species. Literature suggests that the DHBA formation is well correlated with $\mathrm{Cu}$ and Fe concentrations. However, results from the current study do not show any statistically significant correlation between DHBA formations and measured mass emissions rates of metals. The lack of correlation exhibited in this study could be as result of the very low PM mass and consequently the chemical kinetics of the assay unable represents oxidative stress reaction within cells. Results from Table 15 show better correlation for sulfur, potassium, vanadium, iron and cobalt. However, the lack of significance in the correlation demands a larger data set to derive better conclusions. 
Table 15 Pearson Correlation coefficient (R) and level of significance (p) for elemental species Vs. DHBA formation

\begin{tabular}{ccc}
\hline Species & $\mathrm{R}$ & $\mathrm{P}$ \\
\hline $\mathrm{Cu}$ & -0.05 & 0.95 \\
$\mathrm{Mg}$ & 0.37 & 0.63 \\
$\mathrm{Al}$ & 0.45 & 0.55 \\
$\mathrm{Ca}$ & 0.52 & 0.47 \\
$\mathrm{P}$ & -0.29 & 0.72 \\
$\mathrm{~S}$ & $\mathbf{0 . 7 0}$ & $\mathbf{0 . 3 0}$ \\
$\mathbf{K}$ & $\mathbf{0 . 8 0}$ & $\mathbf{0 . 2 0}$ \\
$\mathbf{V}$ & $\mathbf{0 . 7 0}$ & $\mathbf{0 . 3 0}$ \\
$\mathrm{Cr}$ & 0.42 & 0.58 \\
$\mathrm{Mn}$ & 0.57 & 0.43 \\
$\mathrm{Fe}$ & $\mathbf{0 . 8 9}$ & $\mathbf{0 . 1 1}$ \\
$\mathrm{Co}$ & $\mathbf{0 . 7 0}$ & $\mathbf{0 . 3 0}$ \\
$\mathrm{Zn}$ & 0.10 & 0.90 \\
\hline
\end{tabular}

\subsubsection{ALVEOLAR MACROPHAGE ASSAY}

Results of the alveolar macrophage cell based assay, represents the mass of ROS species present in the PM sample as function of fluorescence exhibited by a compound called DCFH-DA. The fluorescence exhibited by the sample is referenced to the fluorescence exhibited by a control substance called zymosan. Hence the fluorescence exhibited by a PM sample will be expressed in units of mass of zymosan per mass of particulate matter. Table 16 shows the results of the alveolar macrophage assay for the different driving cycles for bus 1 and bus 2 .

Figure 5-17 shows the bar plot of the results of the macrophage assay over different driving cycles both CNG buses. Figure 5-18 shows a figure adapted from a study conducted by Verma et al. that has documented the macrophage assay results for various technology diesel and gasoline vehicles (Verma et al., 2010). Comparing the results of the study presented by Verma et al. to the current study it can be seen that the response of heavy-duty natural gas vehicles PM over the UDDS cycle to be comparable to Euro V diesel vehicles. However, since the engine of the buses tested in the current study is a stoichiometrically operated natural gas engine, it would be appropriate to view the toxicity response of the current study with results from a gasoline vehicle, as both vehicles would have a similar after-treatment system configuration 
(Verma et al., 2010). From Figure 5-18 it can be seen that the macrophage assay results observed in this study is one order of magnitude lower than Euro III gasoline vehicles. This could be attributed to the fact that the current study observed ultra low level PM mass emissions and hence the resulting toxicity responses could be characterized by levels of uncertainty. Literature has suggests that the macrophage assay has been developed to be sensitive to PM mass less than $0.1 \mathrm{mg}$. Steady state operation of both vehicles and idle operation of Bus 1 resulted in low PM mass being collected in the sample filter. Although a net filter weight of $0.1 \mathrm{mg}$ was achieved, significant portion of the mass could be a result of positive artifacts due to gas phase adsorption on the T60A20 filter material as opposed to actual mass contribution from particle phase material.

Table 16 Results of macrophage assay for non-denuded particulate matter

\begin{tabular}{|c|c|c|c|c|c|c|c|}
\hline Test Cycle & $\begin{array}{c}\text { Total } \\
\text { Sampling } \\
\text { Time } \\
\text { (hrs) }\end{array}$ & $\begin{array}{c}\text { Total } \\
\text { Distance } \\
\text { (miles) }\end{array}$ & $\begin{array}{c}\text { Total } \\
\text { Non- } \\
\text { Denuded } \\
\text { PM (mg) }\end{array}$ & $\begin{array}{c}\text { Non- } \\
\text { Denuded } \\
\text { Total PM } \\
\text { (mg/mi) }\end{array}$ & $\begin{array}{c}\text { Non- } \\
\text { Denuded } \\
\text { Total PM } \\
\text { (mg/hr) }\end{array}$ & $\begin{array}{c}\text { Non- } \\
\text { Denuded } \\
\text { PM ROS } \\
\text { activity } \\
\text { ( } \mu \text { g of } \\
\text { Zymosan } \\
\text { units) }\end{array}$ & $\begin{array}{c}\text { Non- } \\
\text { Denuded } \\
\text { PM ROS } \\
\text { activity ( } \mu \mathrm{g} \\
\text { of } \\
\text { Zymosan } \\
\text { units/mg } \\
\text { of PM) }\end{array}$ \\
\hline Bus 1 UDDS & 6.18 & 231.6 & 109.87 & 0.47 & 17.77 & 297030.19 & 2703.47 \\
\hline Bus1 $45 \mathrm{MPH}$ & 8.00 & 360.2 & 38.81 & 0.11 & 4.85 & 0 & 0.00 \\
\hline Bus 1 Idle & 12.0 & NA & 29.21 & NA & 2.43 & 108341.62 & 3709.06 \\
\hline Bus 2 UDDS & 7.07 & 183.0 & 231.22 & 1.26 & 32.72 & 99801.49 & 431.63 \\
\hline Bus $245 \mathrm{MPH}$ & 11.0 & 495.8 & 49.62 & 0.10 & 4.51 & 30602.34 & 616.73 \\
\hline Bus 2 Idle & 6.00 & NA & 199.52 & NA & 33.25 & 278701.09 & 1396.86 \\
\hline
\end{tabular}




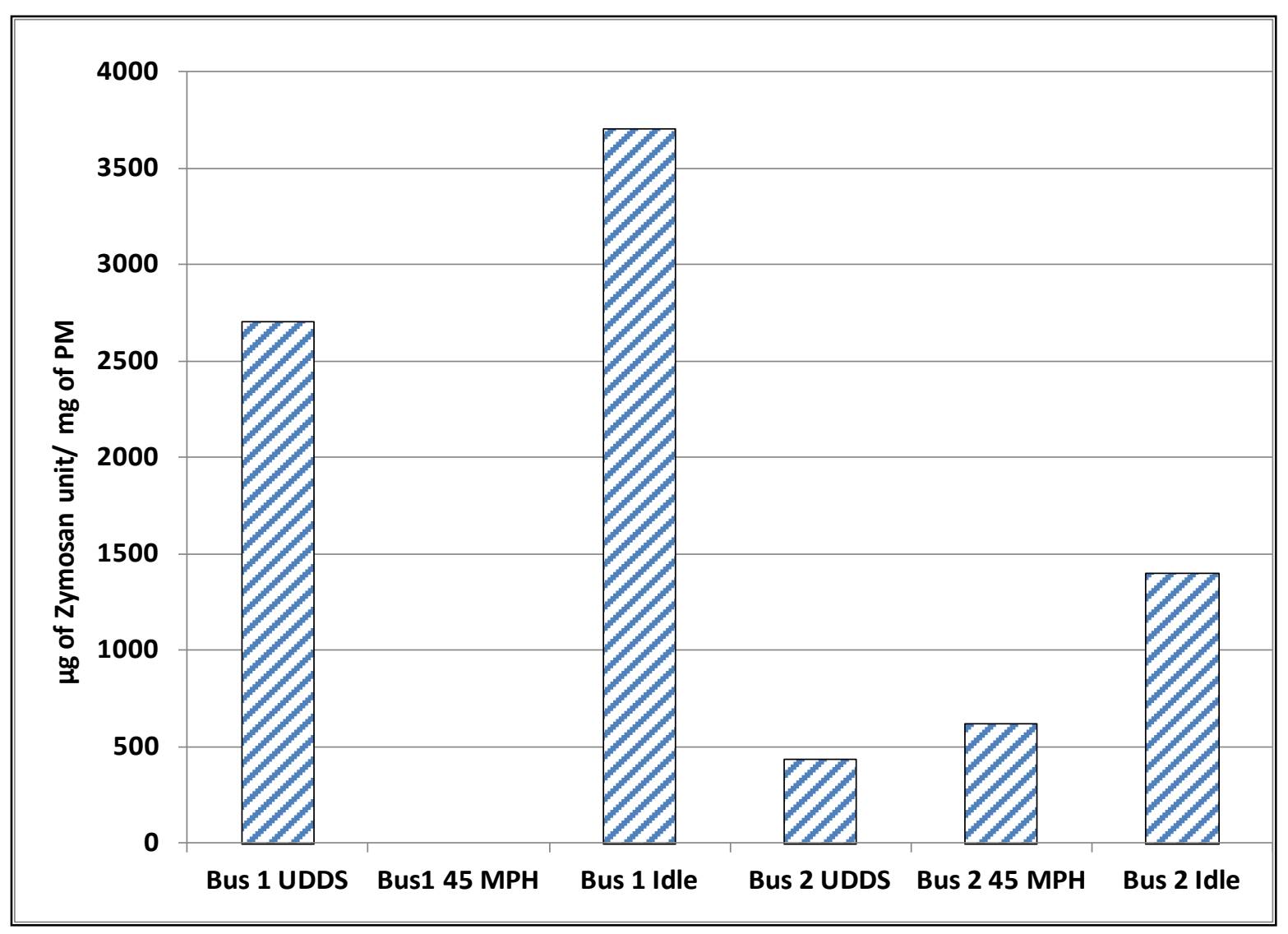

Figure 5-17 PM mass specific macrophage ROS activity over different driving cycles

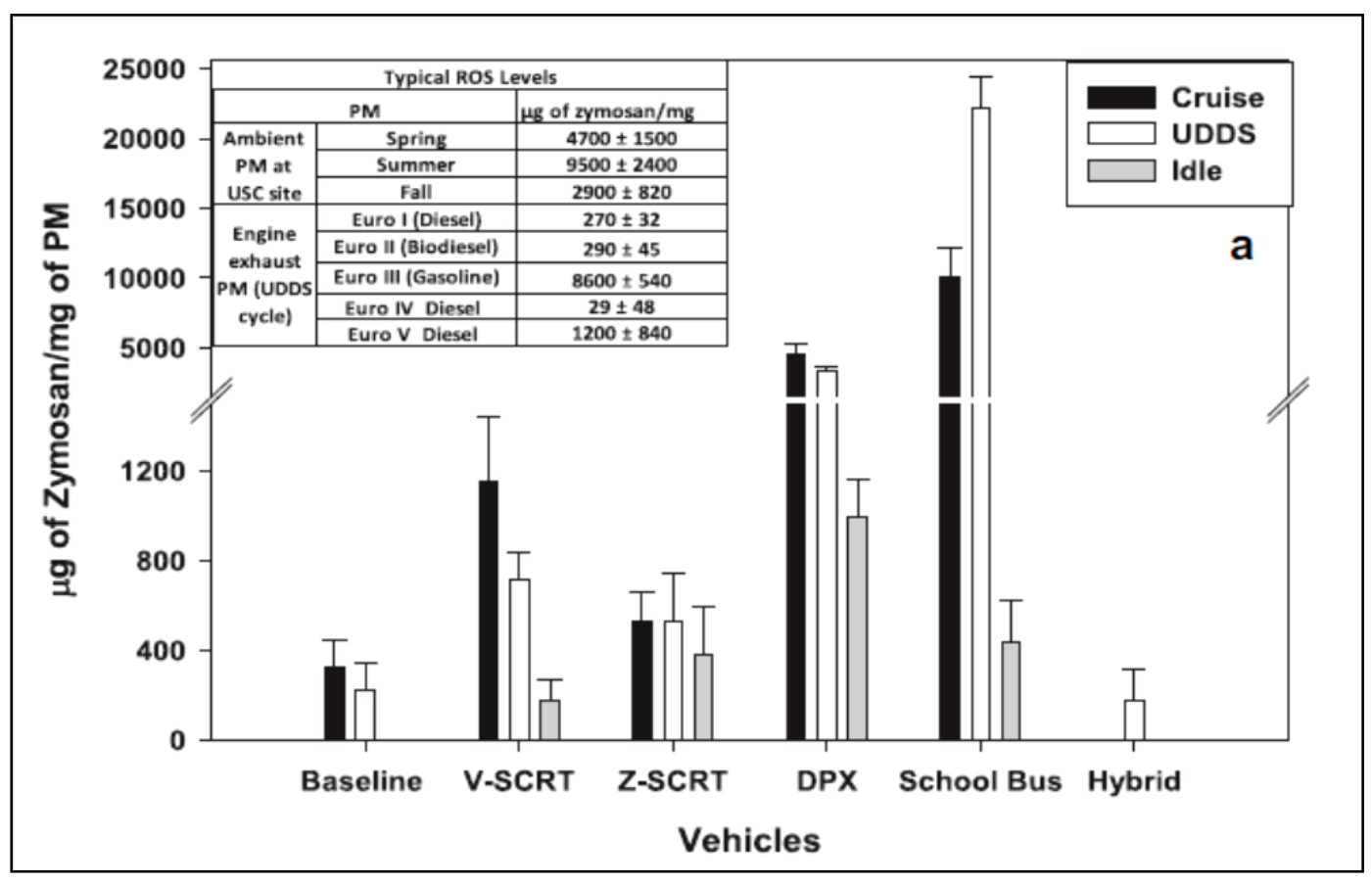

Figure 5-18 PM mass specific macrophage ROS activity of different technology vehicles and ambient PM (Verma et al., 2010) 


\subsubsection{ELEMENTAL CARBON/ORGANIC CARBON COMPOSITION VS. MACROPHAGE ROS ACTIVITY}

This section presents the analysis of the effect of EC/OC on the observed macrophage ROS activity. Since, the macrophage ROS assay utilizes living rat alveolar tissues, the response provided by this assay can be viewed as an actual dose vs. response characteristic of the elemental and organic carbon mass of the PM samples. However, no findings in literature document the relationship of EC/OC mass to the observed ROS activity. Literary knowledge of the response of macrophage ROS to heavy-duty PM has been illustrated only for metals and transition metal composition in PM. This could be due to the fact that the assay is specifically more responsive to water soluble compounds, since aqueous suspensions of the PM are administered to the cell cultures.

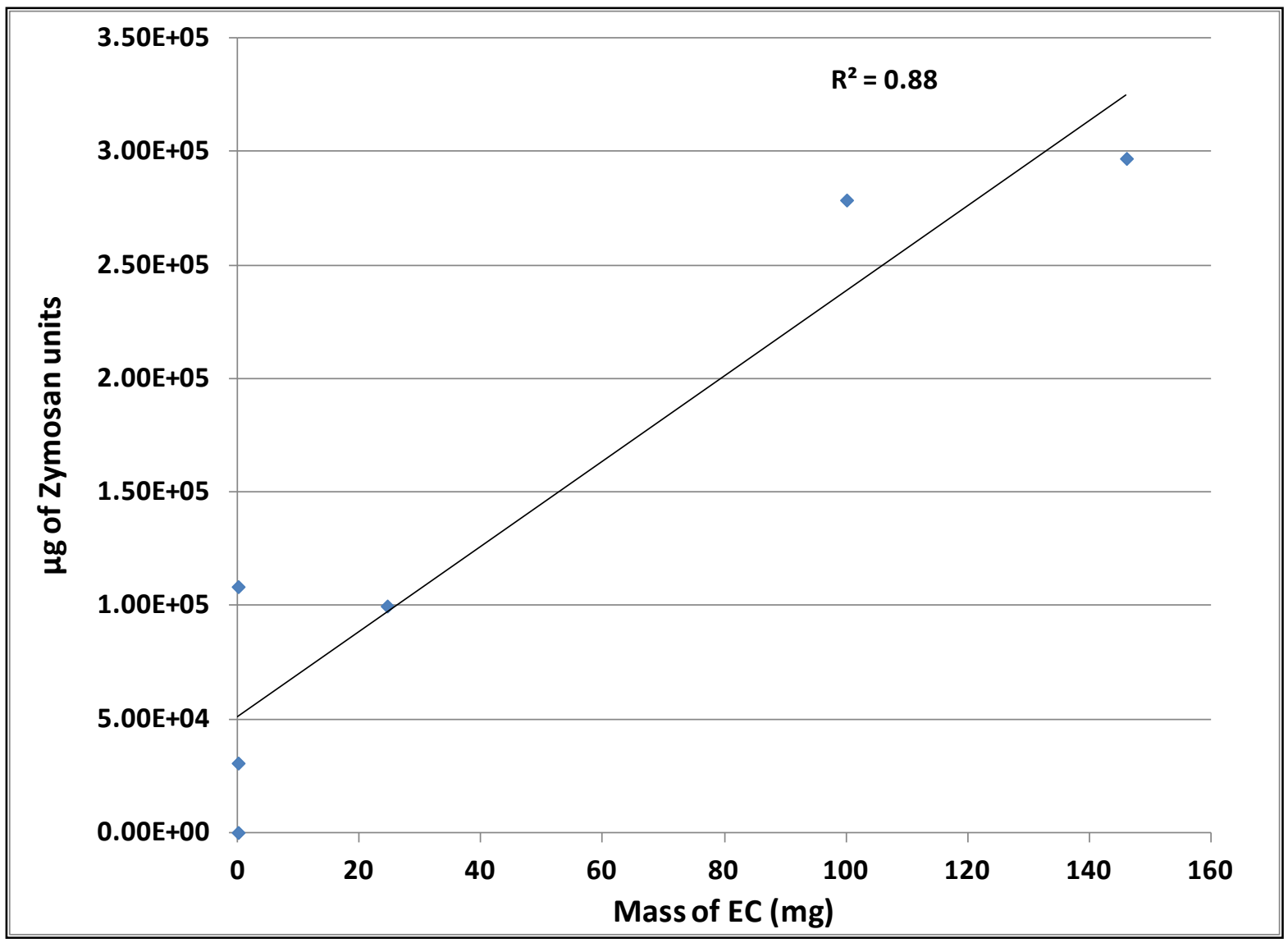

Figure 5-19 Scatter plot of macrophage ROS activity Vs. elemental carbon mass 
Figure 5-19 shows the relationship between EC mass and observed macrophage ROS activity of the non-denuded PM. Table 17 below shows the Pearson correlation coefficient and level of significance for the correlation analysis of EC and OC mass to ROS macrophage activity. The results shows statistically significant correlation between EC mass and macrophage ROS activity $(R=0.94$ and $p=0.004)$. However, it is to be noted that the $E C$ mass for 3 out the 6 data points were reported to be zero. Since, there is lack of literary evidence to corroborate the findings of this study, a greater spread in the EC mass could have provided a better confidence in presenting the correlation between EC mass and macrophage ROS activity. Natural gas PM has characteristically been devoid of EC fraction, and the major contribution to elemental carbon has been from in cylinder combustion of lubrication oil. The correlation analysis of organic carbon mass resulted in correlation coefficient of $R=0.55$ with $p=0.25$, suggesting no statistical significance in the observed correlation. One of the drawbacks in quantifying OC composition of heavy-duty natural gas PM is the possibility of results being skewed by positive gas phase adsorption artifacts on the pre-fired quartz filters.

Table 17 Pearson Correlation coefficient (R) and level of significance (p) for EC/OC Vs. ROS macrophage activity of non-denuded PM

\begin{tabular}{ccc}
\hline Species & $\mathrm{R}$ & $\mathrm{P}$ \\
\hline $\mathrm{EC}$ & 0.94 & 0.004 \\
$\mathrm{OC}$ & 0.55 & 0.25 \\
$\mathrm{PAH}$ & & \\
$(\mathrm{MW}<200)$ & -0.50 & 0.30 \\
\hline
\end{tabular}

A correlation analysis of EC composition vs. denuded PM resulted in poor correlation coefficient $(R=0.39, p=0.43)$. This could be due to the fact that the EC mass collected on the denuded filters were so negligible and the presence of other compounds such as metals could have masked the activity of EC.

Correlation analysis between PAH compounds with molecular weight less than 200 and the ROS activity of non-denuded PM resulted in a negative correlation with no statistical significance. All PAH compounds are insoluble in water and the solubility decreases with 
increasing molecular weight. PAH compounds have previously not been observed to correlate well with macrophage ROS activity (Biswas et al., 2009a). PAH compounds are most often bound with other particulate matter composition such as soot or elements. Hence, the influence of PAH compounds in producing ROS can be viewed as a combined effect of the PAH and its substrate that it is adsorbed on to. Alveolar macrophage assay utilizes ionized water to extract particulate matter from the sample filters and to subsequently introduce it into the cell cultures. PAH compounds having very low levels of water solubility could have resulted in lesser extraction rates from the filter and consequently a statistically insignificant correlation.

\subsubsection{ELEMENTAL COMPOSITION VS. MACROPHAGE ROS ACTIVITY}

Table 18 below shows the Pearson correlation results between elemental composition and macrophage ROS activity detected from denuded and non-denuded PM. The results indicate statistically significant correlation coefficients for the highlighted elements in the table with the ROS activity detected from the denuded PM. The metals that exhibited significant correlation with the ROS activity of the denuded PM resulted in lower correlation coefficient with nondenuded PM. This trend could be attributed to a possible presence of a volatile coating over the elemental particles, thereby preventing the interaction of the alveolar cell tissues and the elemental fraction. As stated earlier, PAH compounds are characterized by low vapor pressures and hence tend to condense on EC or elemental particulate matter fraction. This semi-volatile coating over soot or elemental composition could change the toxicity nature of that particular fraction. Previous studies by Verma et al. have shown the characteristic transition metals to generate ROS through the macrophage ROS assay (Verma et al., 2010). Literature does not illustrate the effect of PAH in the generation ROS through this particular assay. Also the results of the current study indicate no significant correlation of ROS activity with PAH emissions in the exhaust. Lesser correlation of non-denuded PM ROS activity with elemental composition could be a result of lower either a low sample extraction rate from the filters as result of insolubility of $\mathrm{PAH}$ with water and or a layer of $\mathrm{PAH}$ compounds over the elemental fraction possibly preventing the interaction of elemental fraction and alveolar tissue cells. 
Table 18 Pearson Correlation coefficient (R) and level of significance (p) for elemental species vs. macrophage ROS activity for denuded and non-denuded PM

\begin{tabular}{ccccc}
\hline & \multicolumn{2}{c}{ Denuded PM } & \multicolumn{2}{c}{ Non-Denuded PM } \\
\hline Species & $\mathrm{R}$ & $\mathrm{P}$ & $\mathrm{R}$ & $\mathrm{P}$ \\
\hline $\mathrm{Cu}$ & $\mathbf{0 . 9 7}$ & $\mathbf{0 . 0 0}$ & $\mathbf{0 . 5 2}$ & $\mathbf{0 . 2 8}$ \\
$\mathrm{Mg}$ & 0.28 & 0.59 & 0.06 & 0.90 \\
$\mathrm{Al}$ & 0.27 & 0.59 & 0.06 & 0.90 \\
$\mathbf{P}$ & $\mathbf{0 . 9 9}$ & $\mathbf{0 . 0 0}$ & $\mathbf{0 . 5 6}$ & $\mathbf{0 . 2 5}$ \\
$\mathrm{S}$ & $\mathbf{0 . 7 3}$ & $\mathbf{0 . 0 9}$ & $\mathbf{0 . 3 4}$ & $\mathbf{0 . 5 0}$ \\
$\mathrm{K}$ & 0.30 & 0.55 & 0.07 & 0.88 \\
$\mathrm{Ca}$ & 0.33 & 0.52 & 0.72 & 0.11 \\
$\mathrm{~V}$ & 0.83 & 0.04 & 0.40 & 0.43 \\
$\mathrm{Cr}$ & $\mathbf{0 . 9 9}$ & $\mathbf{0 . 0 0}$ & $\mathbf{0 . 5 5}$ & $\mathbf{0 . 2 6}$ \\
$\mathrm{Mn}$ & 0.49 & 0.32 & 0.20 & 0.69 \\
$\mathrm{Fe}$ & $\mathbf{0 . 9 5}$ & $\mathbf{0 . 0 0}$ & $\mathbf{0 . 4 7}$ & $\mathbf{0 . 3 3}$ \\
$\mathrm{Co}$ & 0.68 & 0.13 & 0.27 & 0.60 \\
Zn & $\mathbf{0 . 9 4}$ & $\mathbf{0 . 0 0}$ & $\mathbf{0 . 4 9}$ & $\mathbf{0 . 3 2}$ \\
\hline
\end{tabular}

The study by Verma et al. provides a comprehensive analysis of the effect of various elements, specifically transition metals in generation of ROS species through this particular assay. $\mathrm{Mn}, \mathrm{Co}, \mathrm{Cr}$, Fe were some of the metals identified by the study to correlate statistically well with ROS activity from the macrophage ROS assay (Verma et al., 2010). The cited literature has documented ROS activity from a DPF-SCR equipped vehicle. Hence, the elemental composition observed in the current study is significantly different from that cited in the literature. The current study reports significant emissions of lubrication oil based elements such as $\mathrm{P}, \mathrm{S}, \mathrm{Zn}$ and $\mathrm{Ca}$. On the contrary the study by Verma et al. have documented lower concentration of lubrication oil based elements and hence lower observed correlation with the ROS activity. The statistically significant correlation exhibited by $\mathrm{P}, \mathrm{Zn}$ and $\mathrm{S}$ with the denuded PM ROS activity suggests that lubrication oil based emissions driving bulk of the toxicity reactions in natural gas engines as opposed to diesel engines, which could be dominated by catalyst performance, DPF efficiencies and elemental emissions from after-treatment devices. 


\subsubsection{MULTIVARIATE REGRESSION FOR MACROPHAGE ROS ACTIVITY}

A multivariate regressions analysis was performed between the combination of elemental mass compositions and the observed macrophage ROS activity to discern the combined contribution of the different elemental mass towards ROS generation. Since a majority of the mass of denuded PM originates from the lubrication oil, $\mathrm{P}$ and $\mathrm{Zn}$ are considered to be the two major factors influencing the ROS activity. In addition to these two elemental fractions $\mathrm{Cr}$, $\mathrm{S}$ and Fe mass was included to test for the regression. The results showed that regressions 1 and regression 2 as shown in Table 19 showed a positive correlation with $R^{2}$ equal to 0.98 and 0.88 respectively. The addition of Fe as a variable resulted in negative correlation contradicting the individual influence of Fe on ROS activity. Hence, the Fe was not considered as variable influencing ROS activity in this case.

Table 19 Multivariate regressions analysis results for elemental composition and macrophage ROS activity

\begin{tabular}{ccccc}
\hline & Factor & Coefficient & Value & R \\
\hline & Constant & $\beta_{\mathrm{o}}$ & 39384 & \\
Regression & $\mathrm{P}$ & $\beta_{2}$ & 1 & 0.98 \\
$\mathbf{1}$ & $\mathrm{Zn}$ & $\beta_{3}$ & 0 & \\
& $\mathrm{Cr}$ & $\beta_{4}$ & -4 & \\
\hline & Constant & $\beta_{\mathrm{o}}$ & 35030 & \\
Regression & $\mathrm{P}$ & $\beta_{1}$ & 0 & 0.88 \\
$\mathbf{2}$ & $\mathrm{Zn}$ & $\beta_{2}$ & 1 & \\
& $\mathrm{~S}$ & $\beta_{3}$ & 0 & \\
\hline
\end{tabular}

Figure 5-20 shows the scatter plot of the predicted macrophage ROS activity to the measured ROS activity. The results show a good fit $\left(R^{2}=0.98\right)$ for a combined influence of $P, Z n$ and $\mathrm{Cr}$ and lower fit $\left(\mathrm{R}^{2}=0.88\right)$ for a combination of $\mathrm{P}, \mathrm{Zn}$ and $\mathrm{S}$. The results suggests that the lubrication oil based emissions in the form of elemental emissions of $\mathrm{P}$ and $\mathrm{Zn}$ together with a possible engine wear metal $\mathrm{Cr}$ are the dominant factors driving the ROS species generation in the alveolar macrophage assay. 


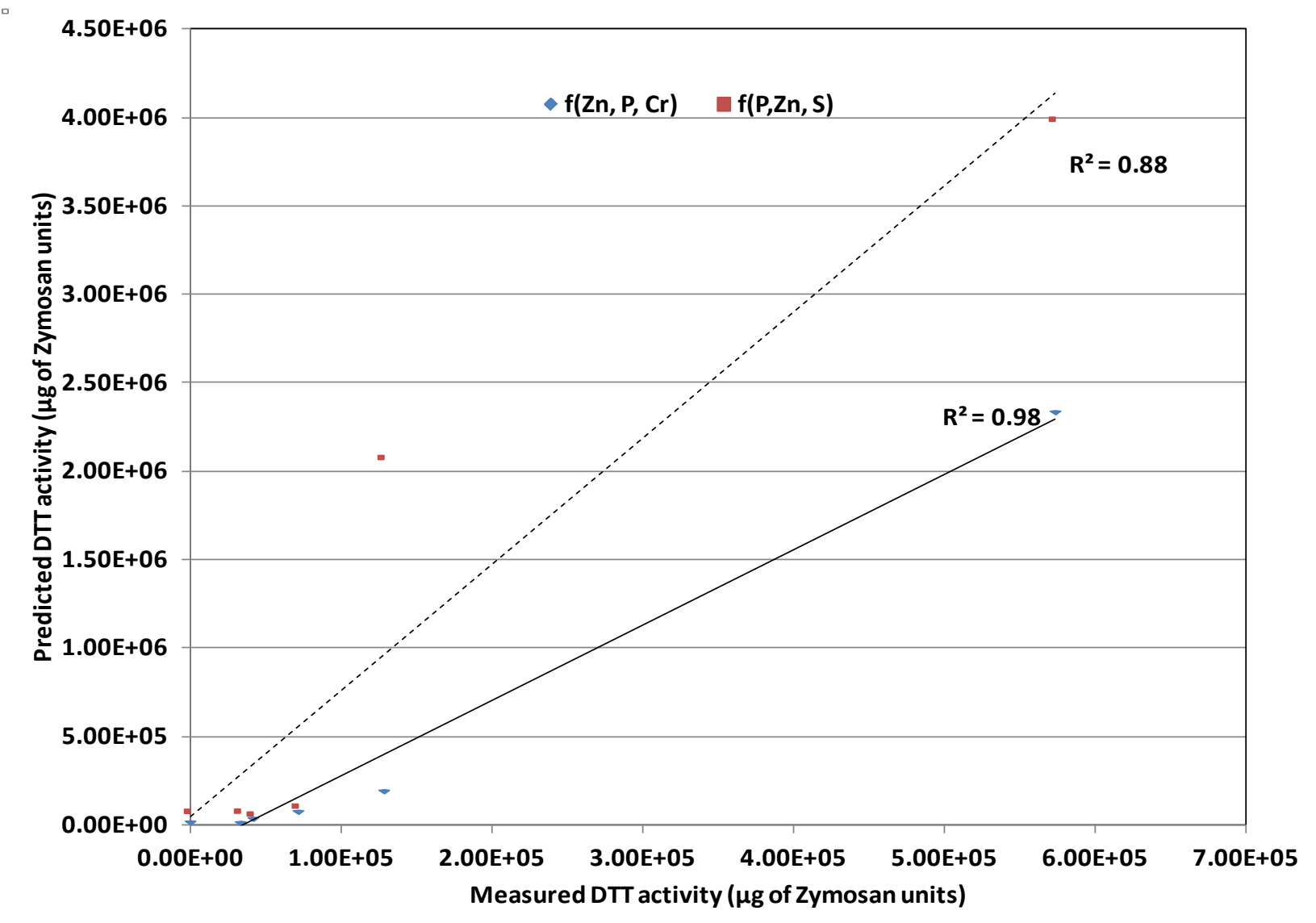

Figure 5-20 Scatter plot of the predicted macrophage ROS activity Vs. measured macrophage ROS activity

\subsection{PARTICLE SIZE DISTRIBUTION ANALYSIS}

Instantaneous particle size distribution and concentration measurements were performed using a TSI EEPS. Primary diluted exhaust was sampled from the CVS and instantaneous dilution ratio calculations were performed as function of exhaust flow and CVS tunnel flow. Secondary dilution was not used, in order to prevent excessive dilution of the exhaust thereby resulting in particle concentrations close to the detection limits of the instrument.

The particle size distribution analysis presented in this section will be further divided into three different bins namely: a) Nanoparticle (NP) with size ranges between 6.04 and $25.5 \mathrm{~nm} \mathrm{~b}$ ) Ultrafine particle (UFP) with size ranges between 29.4 and $107 \mathrm{~nm}$ and c) Fine particle (FP) with size ranges between 124 and $523.3 \mathrm{~nm}$. The bin diameters represent the different channels of the TSI EEPS and hence the diameters represented here are the electrical mobility diameter of the particles. 


\subsubsection{RESULTS OF PARTICLE SIZE DISTRIBUTION ANALYSIS FROM BUS 1}

Figure 5-21 shows the results of UDDS cycle integrated dilution ratio corrected particle size concentration over all the measured size ranges of the TSI EEPS for bus 1 . The illustrated results show the average integrated particle concentration over 4 consecutive UDDS runs with the error bars denoting the maximum and minimum integrated particle concentration values measured.

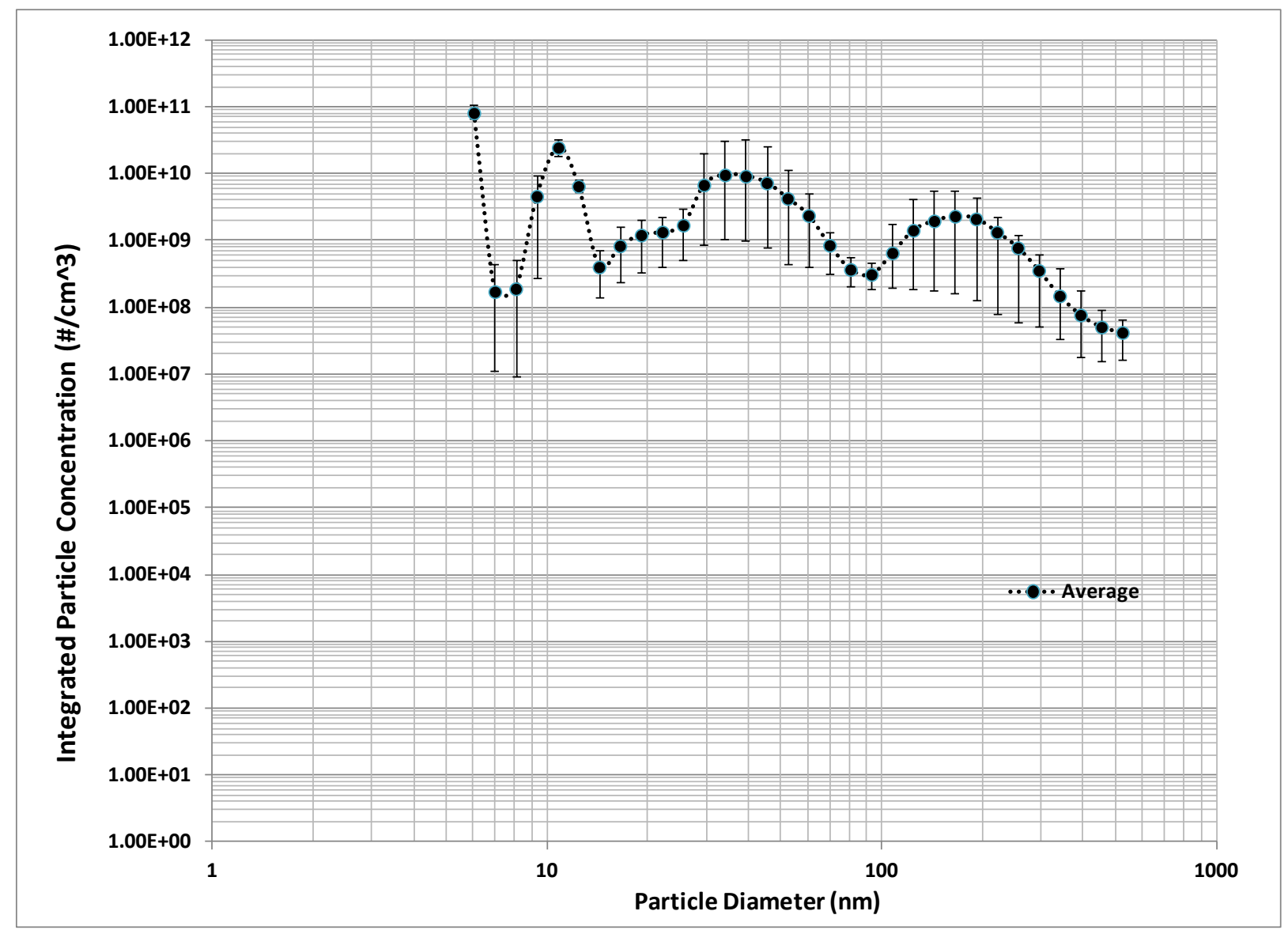

Figure 5-21 Results of integrated particle size distribution and concentration over UDDS cycle from bus 1

The integrated particle size distribution shows significant particle concentration in both nucleation and accumulation mode size ranges. However, the error bars in the accumulation mode size ranges indicate high variability in measured integrated particle concentrations, compared to the nucleation mode integrated particle concentrations. A highly repeatable integrated particle concentration was measured in the $10.8 \mathrm{~nm}$ size range of the EEPS. This method of presenting transient EEPS data would not provide information of the continuously 
changing particle size distribution and concentration measurement over the UDDS cycle, but the variability in the integrated particle concentration data will indicate if the measured particle concentration is characterized by instrument noise.

Contrary to the existing literary knowledge about the unpredictability of the formation of nucleation mode particles, the results from the current study indicates a particle size distribution characterized by lower variation particle number concentration over multiple repeats of a transient driving cycle. This could be attributed to a formation of nanoparticle from sources such as lubrication oil, whose contribution can be considered consistent in comparison to homogenous nucleation of VOCs and catalytic production of nucleation precursors. The high variability in integrated particle number concentration in the accumulation mode size bins indicate the integration of instrument noise in the size range and not actual particles detected. However, the repeatability of the integrated number concentration in the nucleation region indicates particle formation mechanism that is consistent from run to run. The high integrated number concentrations observed in the accumulation region cannot be attributed to particle mass, as they represent only instrument noise.

Figure 5-22 shows the results of the continuous tracking of particle CMD within the three defined size bins of this study. CMD within each particle size bin is plotted together with its corresponding aerosol length (a product of total instantaneous particle number concentration within the size bin and the instantaneous CMD). This method of analysis distributes a tri-modal distribution into three possible mono-modal distributions that can be tracked instantaneously over the course of a transient driving cycle. This method presents an opportunity to graphically visualize the simultaneous evolution of particles within each size bin relative to a time period in the driving cycle. It can be observed from the results shown in Figure 5-22 that the NP size bin reported particle predominantly below $10 \mathrm{~nm}$ with an average CMD over the entire driving cycle of $6.04 \mathrm{~nm}$. For a mono-disperse aerosol of $6.04 \mathrm{~nm}$ an aerosol length of $0.1 \mathrm{~mm} / \mathrm{cm}^{3}$ would represent a particle number concentration of $1.57 \times 10^{4} \mathrm{\#} / \mathrm{cm}^{3}$. Hence it can be seen from figure a) that the measured particle concentration in the NP size bin for the first 400 seconds of the UDDS cycle, was close to the detection limits of the instrument and hence the nucleation mode detected during this time period would be characterized by high levels of 
uncertainty. This time period cannot be considered as warm-up period, due to the fact that the post catalysts exhaust temperature was in the range of $300-500{ }^{\circ} \mathrm{C}$. Figure d) shows the three continuous repeats of the UDDS cycle, and its corresponding post-catalyst exhaust temperatures. As illustrated in Figure 5-22 a) a continuous emission of particles in the $6 \mathrm{~nm}$ size range is observed. The increase in aerosol length in the corresponding indicates a significant increase in total particle concentration within this size range. However, the observed particle CMD and aerosol length do not seem to correlate with transients of the UDDS cycle and tend to be a constant emission rate independent of vehicle operation. For example, periods of idle operation during the UDDS cycle did not result in lower aerosol length values in the NP size bin.
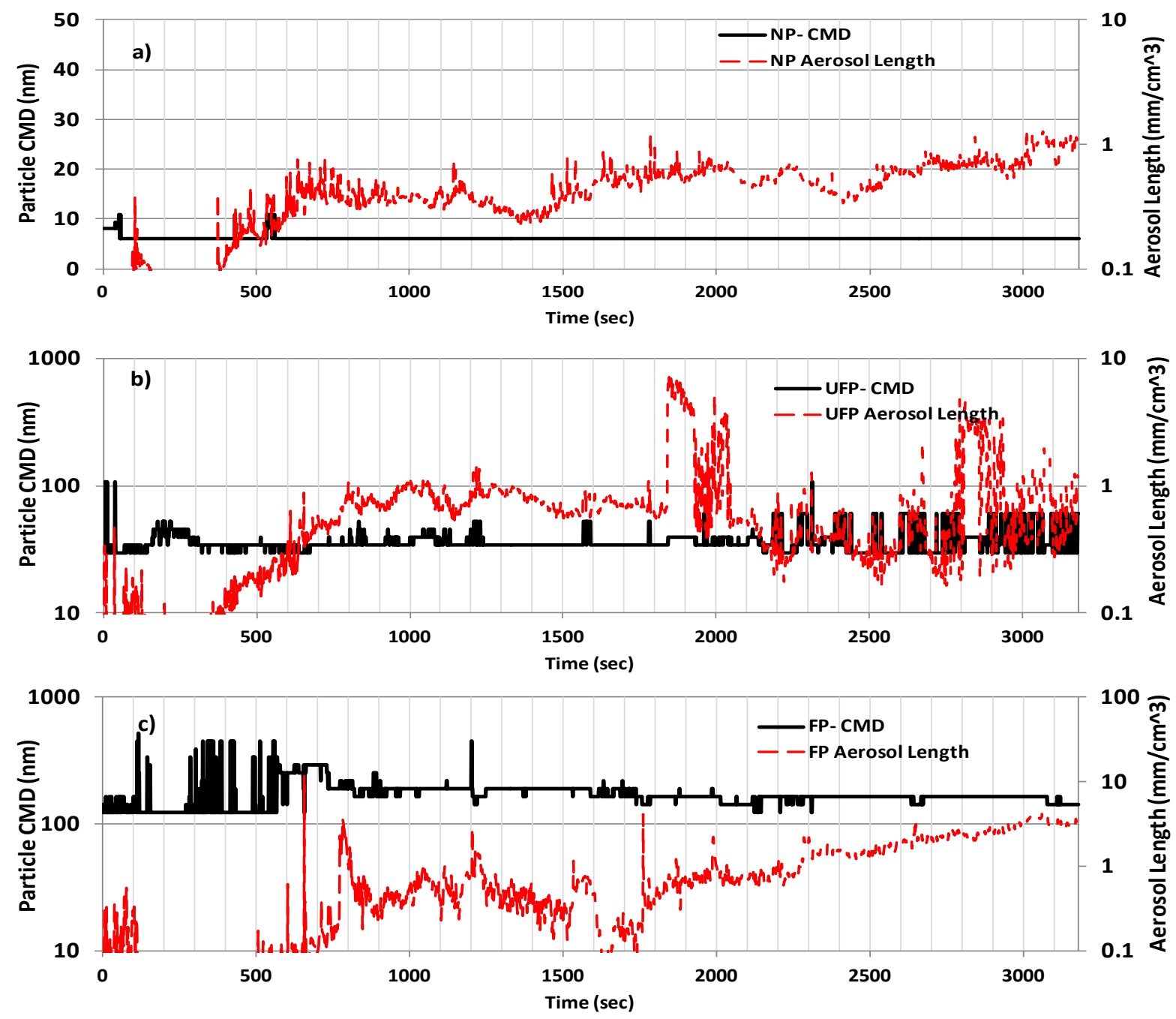

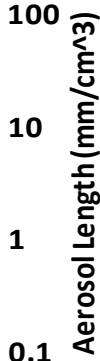




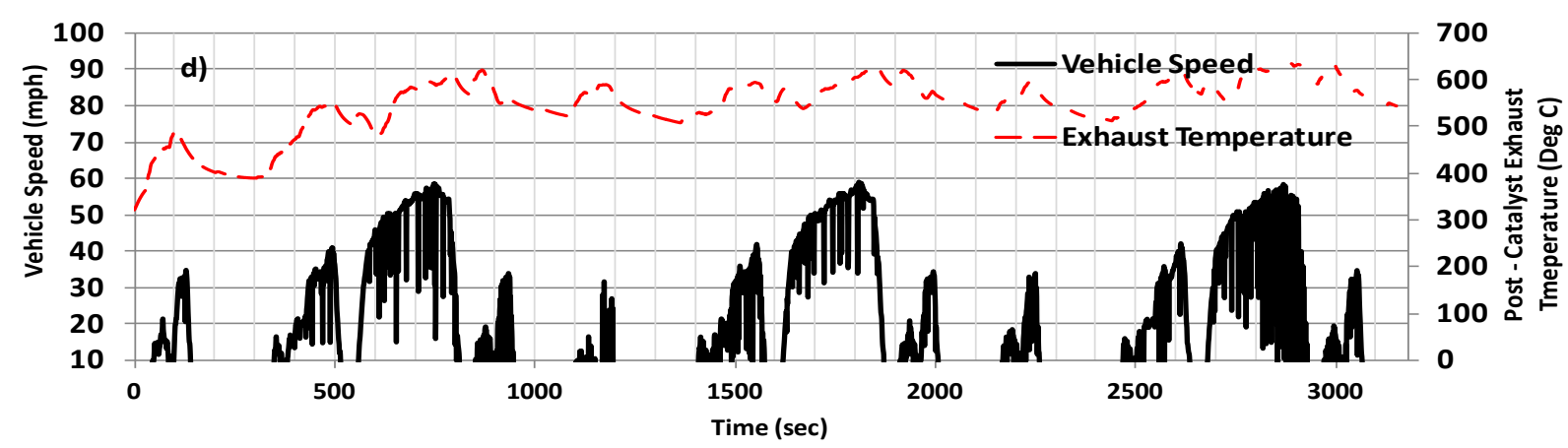

Figure 5-22 Bus 1 a) Transient NP bin CMD and corresponding aerosol length b) Transient UFP bin CMD and corresponding aerosol length c) Transient FP bin CMD and corresponding aerosol length d) UDDS cycle speed trace and post catalyst exhaust temperature

Results shown in Figure 5-22 b) represent the instantaneous CMD values for the UFP size bin and its corresponding aerosol length. The average CMD within this size bin over the entire UDDS cycle was $44 \mathrm{~nm}$. Hence an aerosol length of $1 \mathrm{~mm} / \mathrm{cm}^{3}$ for a mono-disperse aerosol stream with CMD $44 \mathrm{~nm}$ will correspond to a particle concentration of $2.3 \times 10^{4} \# / \mathrm{cm}^{3}$. As illustrated a high variability in the CMD values is observed over the course UDDS cycle. The instantaneous aerosol length calculation for this size range indicate particle concentrations close to the detection limits of the instrument for a major duration of the driving cycle. This indicates high levels of uncertainty in the measured distribution and concentration within this size fraction. The aerosol length calculations also indicate a significant particle contribution with a CMD in the size range of $34-50 \mathrm{~nm}$ between 1500 and $2000 \mathrm{sec}$ time period of the driving cycle. EC emission during the UDDS cycle was observed to be $0.134 \mathrm{mg} / \mathrm{mile}$ and a major fraction of the EC emissions would be comprised within the UFP size bin. Despite the fact that a gaseous fuel, would not contribute to in-cylinder EC formation, certain operating conditions could contribute to entrainment of lubrication oil into the combustion chamber, which could possibly result in EC fraction of the total PM.

Results shown in Figure 5-22 c) represent the instantaneous CMD values for the FP size bin and its corresponding aerosol length. The average CMD in this size bin over the entire driving cycle was $156 \mathrm{~nm}$. Hence an aerosol length of $1 \mathrm{~mm} / \mathrm{cm}^{3}$ for a mono-disperse aerosol stream of $156 \mathrm{~nm}$ will correspond to a total particle concentration of $6.41 \times 10^{3} \mathrm{\#} / \mathrm{cm}^{3}$. As illustrated particle concentration in this size range was close to the detection limits of the instrument, and the CMD reported for a significant duration of the driving cycle (up to 2000 seconds) in this 
chart could only be representing electrometer noise of the TSI EEPS. However, the later segment of the UDDS cycle reports a gradual increase in aerosol length for the reported CMD for this size bin. Since, the FP size bin includes particle size range that is uncharacteristic of CNG exhaust, the resulting CMD at $165 \mathrm{~nm}$ detected with significant particle concentrations could be a result of particle formation from tunnel artifacts such as degassing of adsorbed volatile materials from tunnel walls due to extended high exhaust temperatures or a possible artifact of ammonia emissions from the three-way catalyst. Stoichiometric fuelling of natural gas, together with a three-way oxidation catalyst, has been attributed to catalytic formation of ammonia at rich operating conditions. The formation of ammonia has been linked to the possible water gas shift reactions that could take place on the surface of the catalyst during NOx reduction at rich fuelling conditions (Defoort et al., 2003). Since findings of significant emissions of ammonia from heavy-duty natural gas vehicles was an important outcome of this study which was realized during the final phases of the project. Hence, a setup to quantify ammonia emissions and possibly investigate the influence of ammonia emissions on particle size distribution was not part of the initial test plan.

Figure 5-23 shows the results of the integrated particle size distribution and concentration measurements for the idle operation of bus 1 . The results show a particle size distribution with a predominant nucleation peak observed at a diameter of $8 \mathrm{~nm}$. Integrated peak particle concentration for the observed peak particle diameter was found to be $6.41 \times 10^{9} \mathrm{\#} / \mathrm{cm}^{3}$. An average post catalyst exhaust temperature of 300 Deg $C$ would have been sufficient for the light-off of higher chain hydrocarbons. Hence conditions for particle formation through gas to particle conversion of higher chain volatile organic carbon would not have been prevalent. Also, the EC/OC analysis and VOC speciation reported organic carbon, elemental carbon together and concentrations of VOCs to be below detection limit or at levels observed in tunnel background samples. 


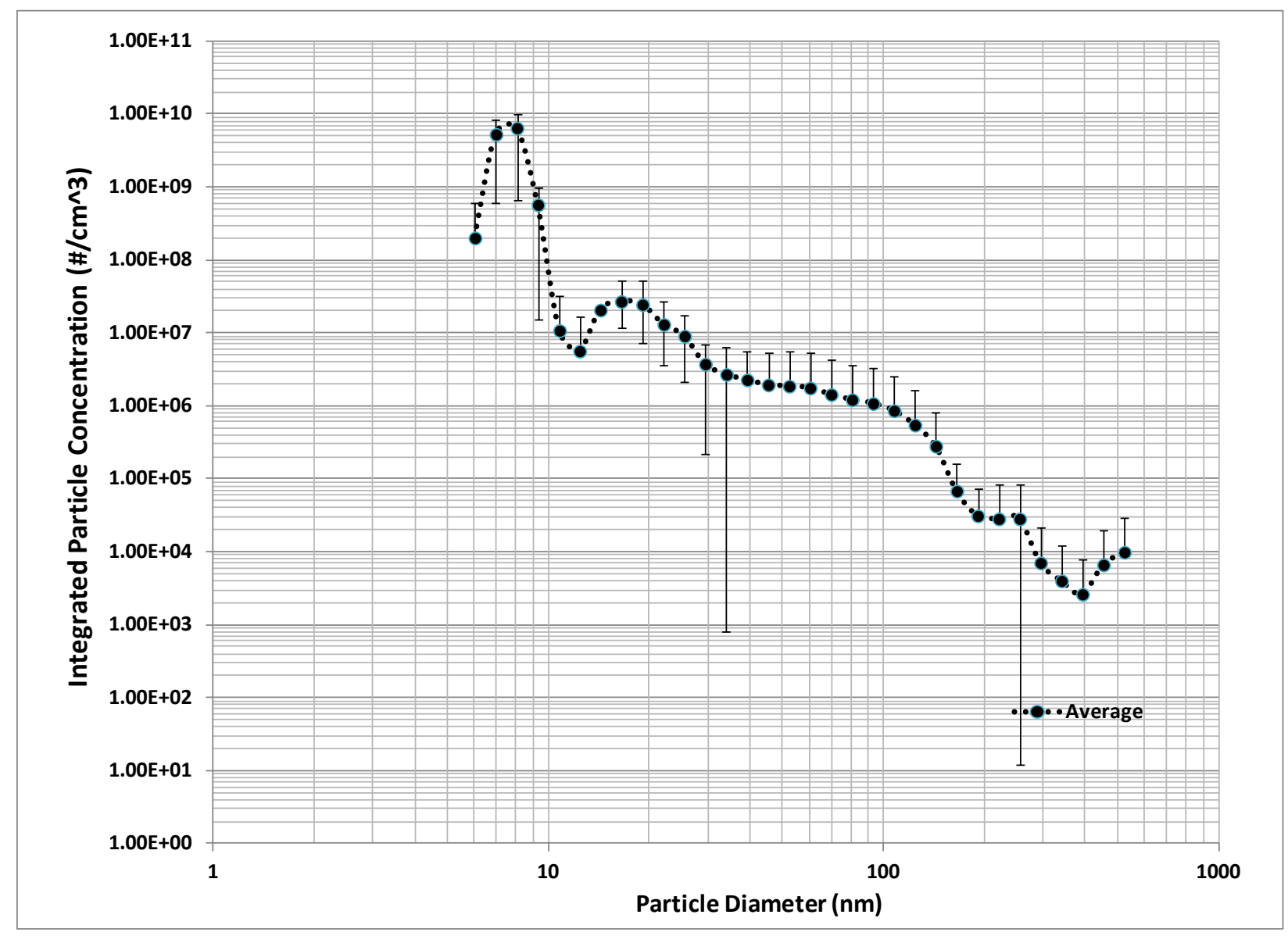

Figure 5-23 Results of integrated particle size distribution and concentration during Idle operation of bus 1

Figure 5-24 shows the integrated particle concentration over the entire size range of the EEPS over the $45 \mathrm{MPH}$ steady state driving cycle. The integrated particle concentration measurement over the entire size range of the EEPS indicates a highly variable distribution and particle number concentration. A consistent particle peak within the nucleation region as observed in both the UDDS and the idle operation is observed for the $45 \mathrm{MPH}$ steady state operation also. However, the nucleation mode particle concentrations observed during the steady state operation was characterized by higher variation in peak particle diameter than the UDDS and idle operation. The average integrated particle concentration results indicate high total particle number concentration in the accumulation mode size ranges. However, the results indicate particle number concentration to be successively decreasing with each consecutive test run. This could be attributed to the possible decrease in sampling artifacts in the CVS tunnel. The average integrated peak particle concentration is found to be $3.12 \times 10^{9} \mathrm{\#} / \mathrm{cm}^{3}$ at a particle 
diameter of $10.9 \mathrm{~nm}$. Results from run 4 indicate no accumulation mode size distribution and a marginal shift in the nucleation mode peak particle diameter towards $6 \mathrm{~nm}$. The results of the steady state mode further, supports our hypothesis of tunnel artifacts contributing to the majority of UFP and FP size bin particle size distribution and concentration. The particles detected as a result of tunnel artifacts could possibly ammonia based salts that could coat the walls of the tunnel during and consequently result in shedding during higher dilution tunnel temperatures. The steady state operation resulted in average post catalyst exhaust temperatures of about $530{ }^{\circ} \mathrm{C}$. Hence, a continuous high temperature exhaust sampling through the CVS could result in shedding of adsorbed material from tunnel walls, which further manifest itself in the UFP and FP size bin of the particle size distribution.

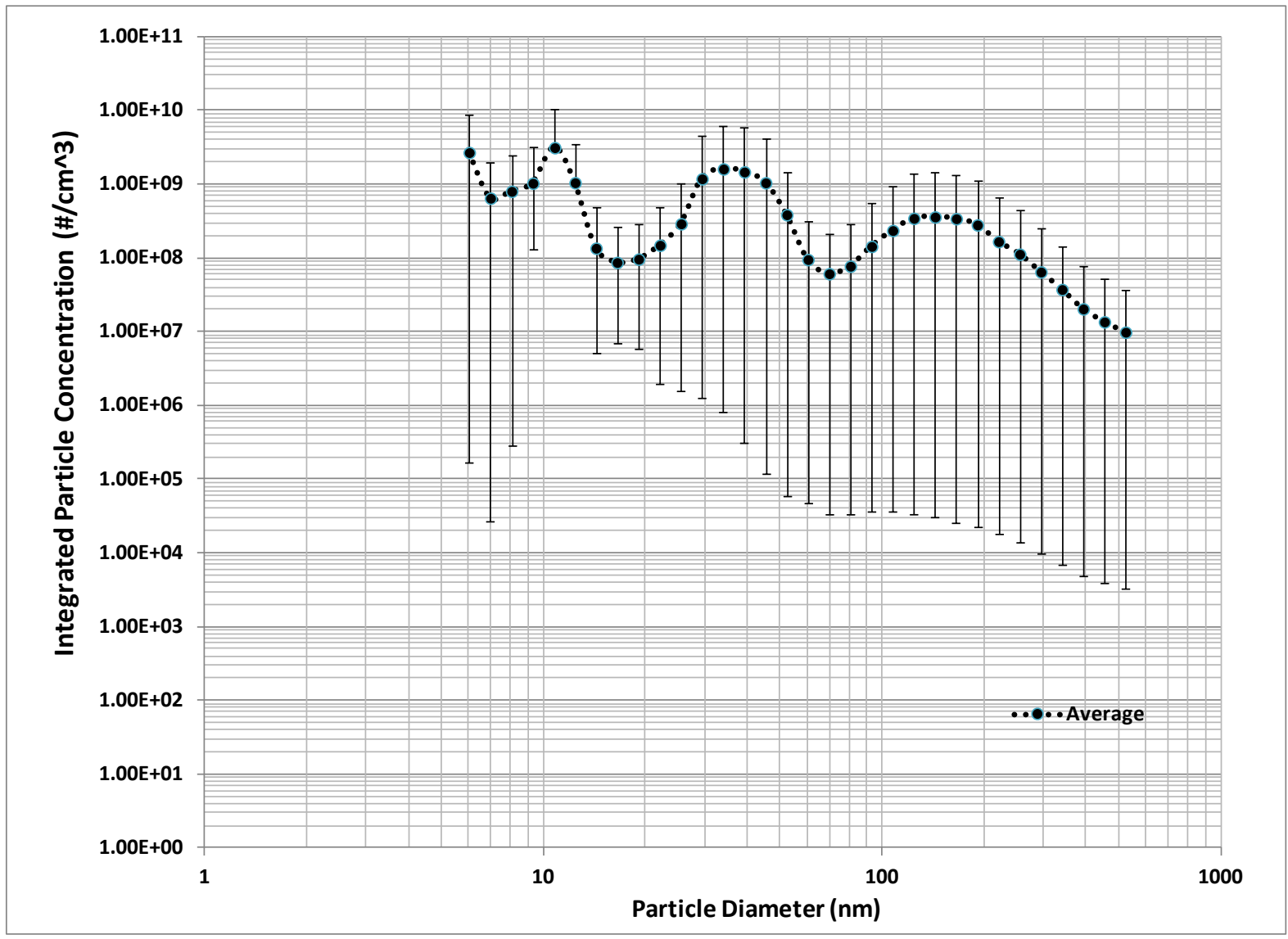

Figure 5-24 Results of integrated particle size distribution and concentration over the steady state 45 MPH cycle from bus 1 
Figure 5-25 a) shows the results of the instantaneous tracking of CMD of particles within the NP size bin with its associated aerosol length. As illustrated particle concentrations in the 10 $\mathrm{nm}$ size range is observed to be relatively repeatable over consecutive runs in comparison to particle concentrations over other size ranges. An aerosol length of $1 \mathrm{~mm} / \mathrm{cm}^{3}$ for a monodisperse aerosol stream of $10 \mathrm{~nm}$ would correspond to a particle concentration of $1 \times 10^{5} \mathrm{\#} / \mathrm{cm}^{3}$, which is an order of magnitude greater than normally observed ambient background particle concentrations. Figure 5-25 b) shows the results of the instantaneous tracking of CMD of particles within the UFP size bin with its associated aerosol length. An aerosol length of 1 $\mathrm{mm} / \mathrm{cm}^{3}$ for a mono-disperse aerosol stream of $107 \mathrm{~nm}$ would correspond to a particle concentration of $1 \times 10^{4} \# / \mathrm{cm}^{3}$. Figure 5-25 b) shows the results of the instantaneous tracking of CMD of particles within the FP size bin with its associated aerosol length. An aerosol length of 1 $\mathrm{mm} / \mathrm{cm}^{3}$ for a mono-disperse aerosol stream of $143 \mathrm{~nm}$ would correspond to a particle concentration of $1 \times 10^{3} \# / \mathrm{cm}^{3}$. Since, the measured concentration within both the UFP and FP size bin is close to the detection limits of the instrument, the detected CMDs could be characterized by higher levels of instrument noise.

If the particles in this size bin are assumed to be volatile in nature, then we can suggest that a steady state operation, contributes significant amount of nucleation precursors that assists in the formation of $10 \mathrm{~nm}$ particles. However, the results of the VOC, PAH and carbonyl suggest possible nucleation precursors and particle phase volatile organic compounds to be below detection limits of the analytic procedure. EC/OC analysis reported no elemental carbon, but $0.203 \mathrm{mg} / \mathrm{mi}$ of organic carbon mass that could be attribute to possible lower chain hydrocarbons getting adsorbed onto the pre-fired quartz filters. Figure 5-25 d) shows a consistent exhaust temperature averaging at $526{ }^{\circ} \mathrm{C}$. The catalytic light-off temperatures of hydrocarbons decrease with lower carbon content. Hence methane with one carbon atom has the highest light-off temperature close to $450{ }^{\circ} \mathrm{C}$. Hydrocarbons that are usually involved in particle formation are higher chain hydrocarbons with low vapor pressures that can readily undergo gas to particle conversion. However, with high exhaust temperatures as in the steady state operation, one would expect to observe a complete methane and ethane based hydrocarbon content in the exhaust, in contrast to BTEX or other PAH compounds that would 
have had catalytic reduction efficiencies of close to $100 \%$ at such high temperatures. The presence of a continuous nucleation mode size distribution peaking at $10 \mathrm{~nm}$ in diameter, suggests the possibility of non-volatile compounds that could be produced as result of lubrication oil entry into the combustion chamber. Figure 5-25 d) also shows the engine percent load through the steady state operation. As illustrated a constant engine load of about $35 \%$ of peak torque with momentary loading due to auxiliary systems such as radiator fan, and alternator are observed. Hence, the contribution of the nucleation mode particles must be independent of the engine operation and must be prevalent through the entire operating regime of the engine. Although, the entry of lubrication oil through the piston rings into the combustion chamber can be viewed as function of engine load, it is to be noted that modern heavy-duty diesel and natural gas engines have employed crank case ventilation to be inducted back into the engine. As result, we could be observing a continuous intake of oil mist and its associated decomposition by products entering into the combustion chamber. 

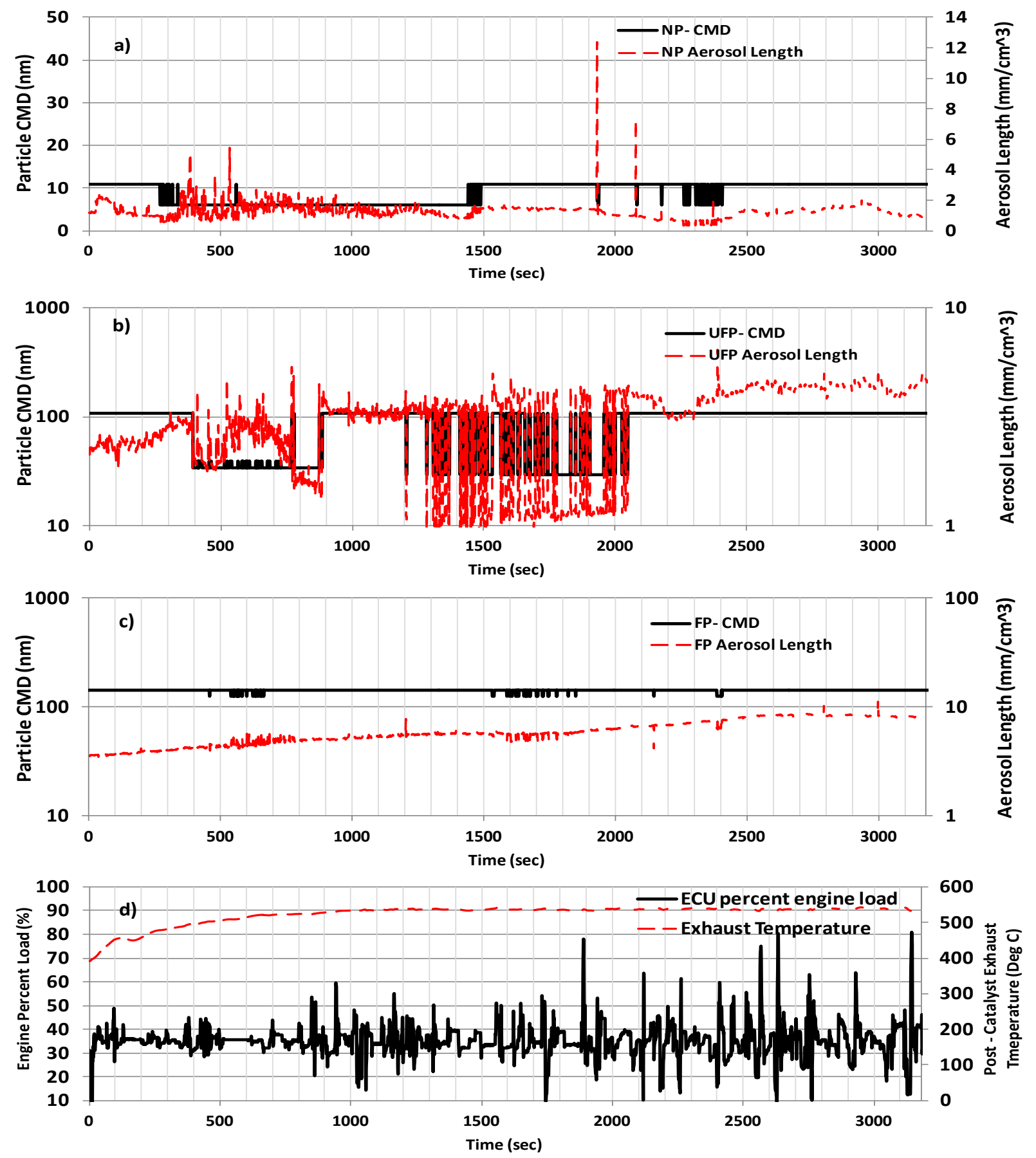

Figure 5-25 Bus 1 steady state $45 \mathrm{MPH}$ a) NP bin CMD and corresponding aerosol length b) UFP bin CMD and corresponding aerosol length $\mathrm{C}$ ) FP bin CMD and corresponding aerosol length d) engine percent load and post catalyst exhaust temperature 


\subsubsection{RESULTS OF PARTICLE SIZE DISTRIBUTION ANALYSIS FROM BUS 2}

Figure 5-26 shows the average integrated particle size distribution and concentration measurements from bus 2 over the UDDS cycle. The results show a highly variable particle size distribution, in contrast to the particle size distribution observed from bus 1 . A distinct nucleation mode size peak is observed at a CMD between 8 and $10 \mathrm{~nm}$. The particle size distribution from bus 2 is significantly different from that observed from bus 1 , with respect to the significant nucleation mode size distribution observed over the entire length of the UDDS cycle. Similar, to the results in bus 1 a significant particle size distribution is observed in the fine particle range ( $\mathrm{Dp}>100 \mathrm{~nm}$ ). However, the particle concentration in this size range is also seen progressively decreasing with successive hot starts, lending support to the assumption of possible tunnel artifacts contributing to particle count in this size range.

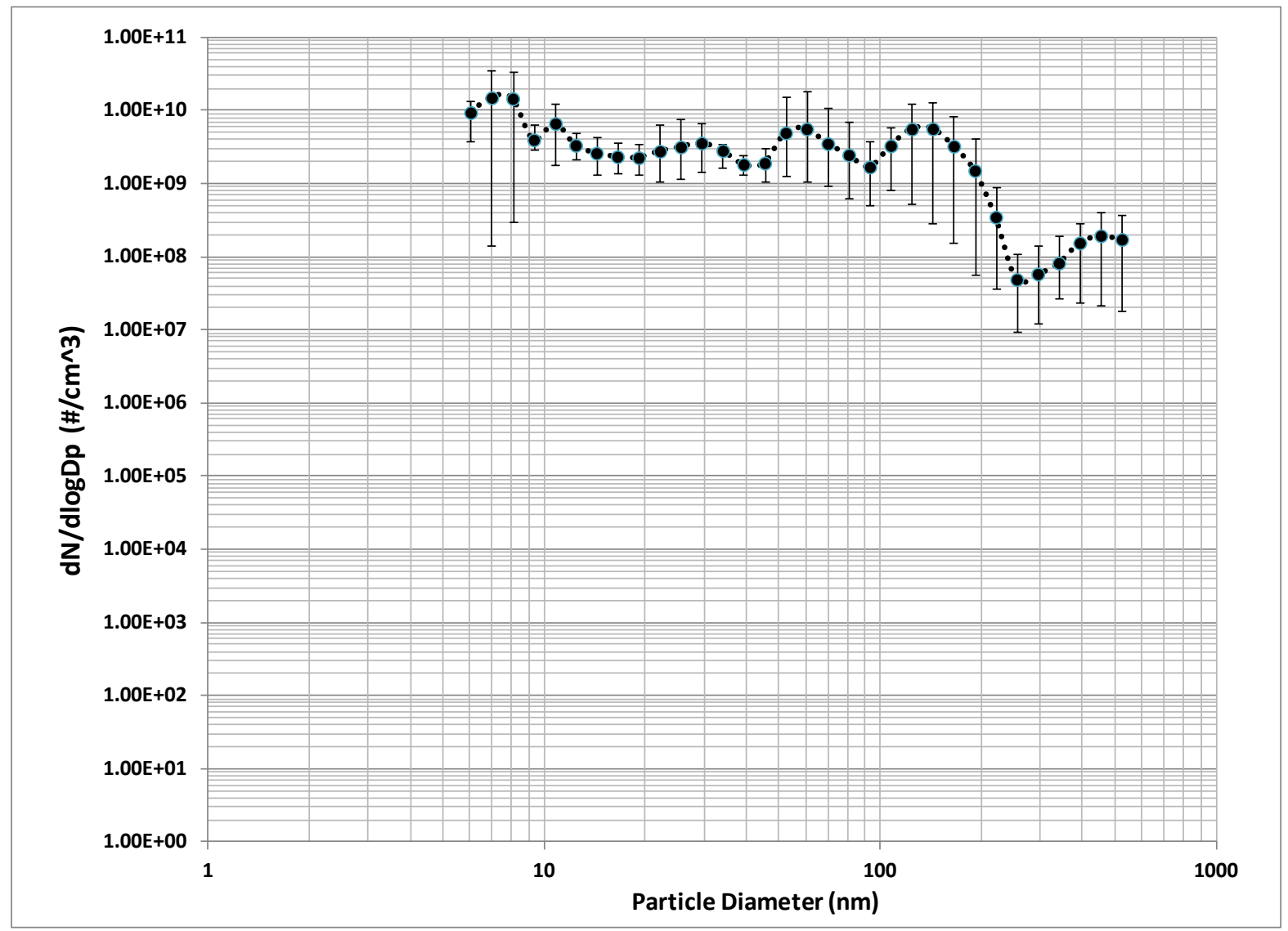

Figure 5-26 Results of integrated particle size distribution and concentration over the UDDS cycle of bus 2 

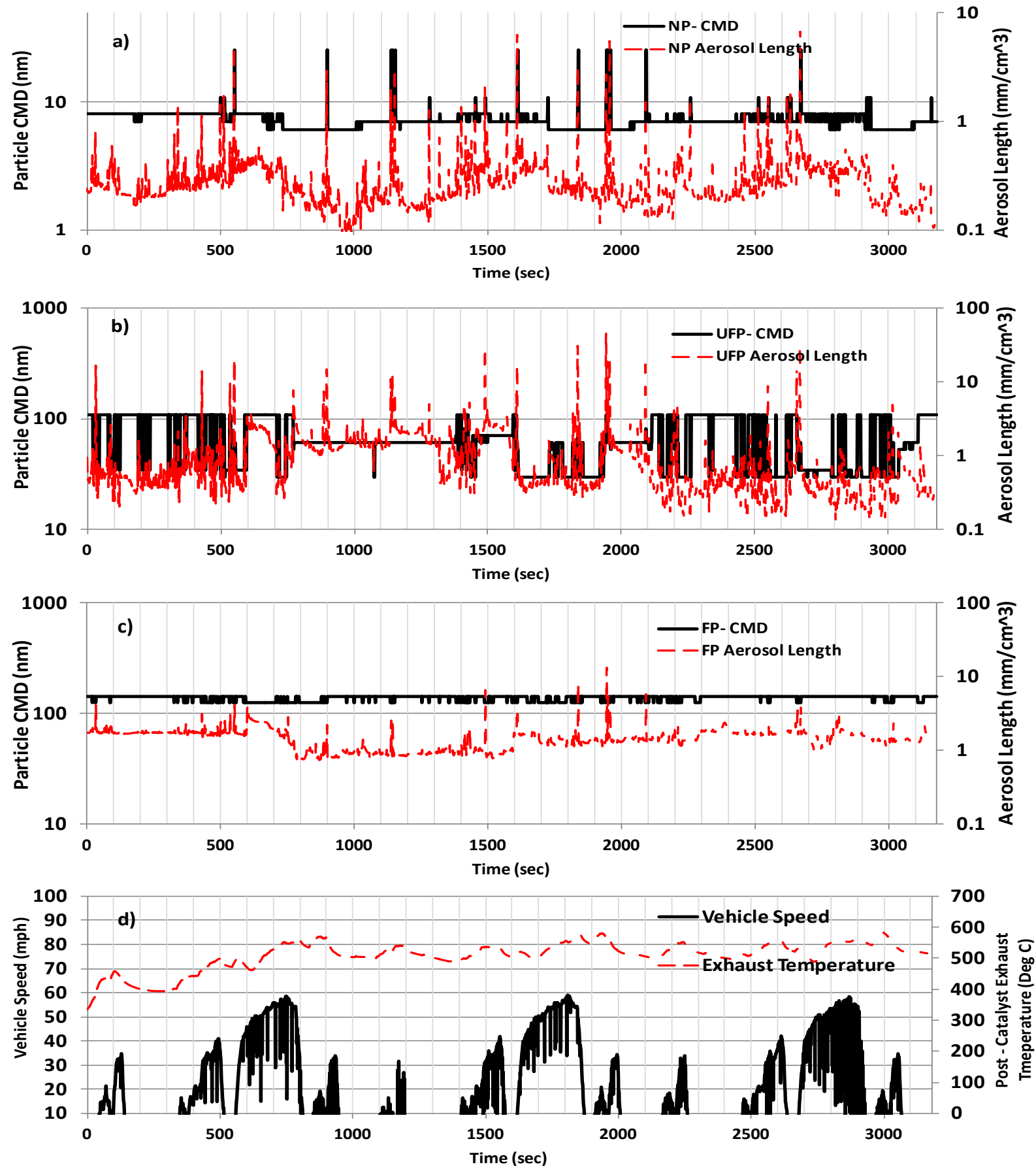

Figure 5-27 Bus 2 a) Transient NP bin CMD and corresponding aerosol length b) Transient UFP bin CMD and corresponding aerosol length c) Transient FP bin CMD and corresponding aerosol length d) UDDS cycle speed trace and post catalyst exhaust temperature

Figure 5-27 a) represents the instantaneous CMD tracking of particles within the NP size bin and its associated aerosol length. The average CMD within this size bin over the duration of the UDDS cycle was observed to be $7 \mathrm{~nm}$ with an average aerosol length of $0.29 \mathrm{~mm} / \mathrm{cm}^{3}$. For a 
mono-disperse particle stream of $7 \mathrm{~nm}$ an aerosol length of $0.29 \mathrm{~mm} / \mathrm{cm}^{3}$ would correspond to a particle number concentration of $4.14 \times 10^{4} \# / \mathrm{cm}^{3}$. As a result on an average particle number concentrations detected in this size bin were close to the detection limits of the instrument and as result could be characterized by higher levels of electrometer noise of the TSI EEPS. Figure 5-27 b) represents the instantaneous CMD tracking for particles within the UFP size bin and its associated aerosol length. The results showed a highly variable CMD values averaging at $68 \mathrm{~nm}$ were detected through the duration of the UDDS cycle. The CMD tracking reveal particle diameters in the size range 29 to $107 \mathrm{~nm}$ within this size bin. Average aerosol length for the UDDS driving cycle was observed to be $1.16 \mathrm{~mm} / \mathrm{cm}^{3}$. For a mono-disperse particle stream of $60 \mathrm{~nm}$ and aerosol length of $1.16 \mathrm{~mm} / \mathrm{cm}^{3}$ would correspond to a particle concentration of 1.76 $X 10^{4} \mathrm{\#} / \mathrm{cm}^{3}$. Hence, the detected concentration on an average was close to the detection limits of the instrument. However, periods of acceleration and idle operation resulted in particles with CMD of $60 \mathrm{~nm}$ with concentrations significantly greater than the instrument noise levels. Figure 5-27 c) represents the instantaneous CMD tracking for particles within the FP size bi with its associated aerosol length. The results show a constant particle CMD in the range of $138 \mathrm{~nm}$, the calculated aerosol length of $1 \mathrm{~mm} / \mathrm{cm}^{3}$ over the entire cycle duration, translates to a particle concentration of $7.25 \times 10^{3} \# / \mathrm{cm}^{3}$. Hence the detected particle CMD in this size range would be characterized by higher levels of instrument noise, and as a result cannot be associated with combustion generated particulate matter concentration. The exhaust temperatures (Figure 5-27 d)) through the duration of the cycle is consistently over 400 Deg C, suggesting conditions for optimal catalytic activity towards hydrocarbons and other organic fractions, capable of particle formation. The absence of a pronounced nucleation mode size distribution, suggests a lesser influence of lubrication oil over the exhaust nano particle formation of this vehicle over the UDDS cycle.

Figure 5-28 shows the results of the integrated particle size distribution and concentration measurement during idle operation from bus 2. Data was collected for an hour long idle cycle, and the distribution shown below represents the particle concentration integrated over the entire duration of the tests. The particle size distribution observed during the idle operation 
was observed to be very repeatable and a broad distribution encompassing particle diameters in the range of the NP, UFP and the FP size bin.

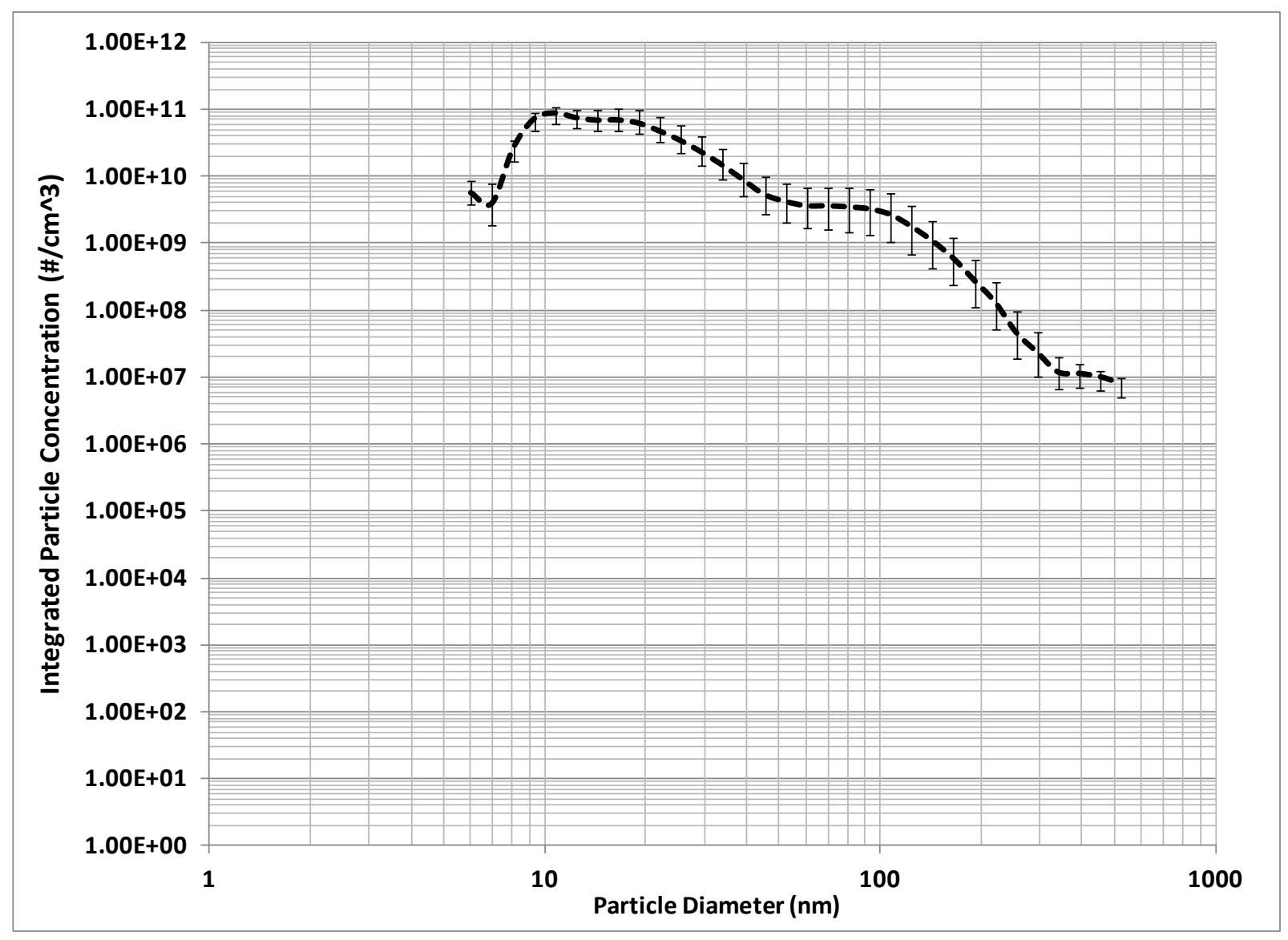

Figure 5-28 Results of integrated particle size distribution and concentration over Idle operation of bus 2

The particle sizing results obtained during the idle operation shows a very interesting distribution that measured particles with significant concentration almost through the entire particle range of the TSI EEPS. The very low run to run variation in the distribution and significant particle concentrations detected in all size ranges, suggest a factor that contributed to consistent particle formation mechanism. Bus 2 idle emissions reported the highest mass loading on the gravimetric filter on a time specific unit. Figure 5-29 shows the coloration of the gravimetric filter samples after 9 hours of sampling during the idle operation. As illustrated it can be seen that the idle operation appears to have produced significant mass of solid fraction of PM. 


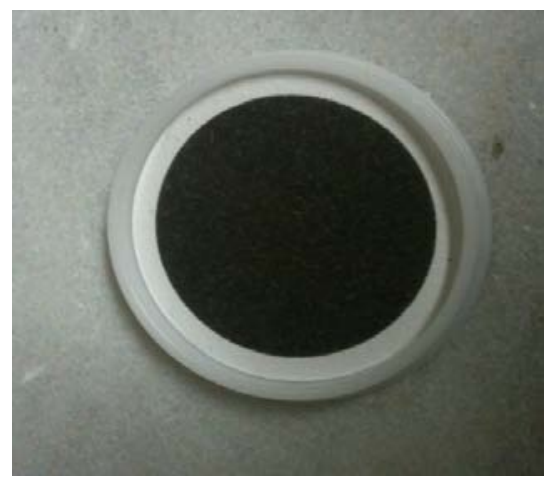

Figure 5-29 Gravimetric filter sample after 9 hours of PM sampling during the idle operation
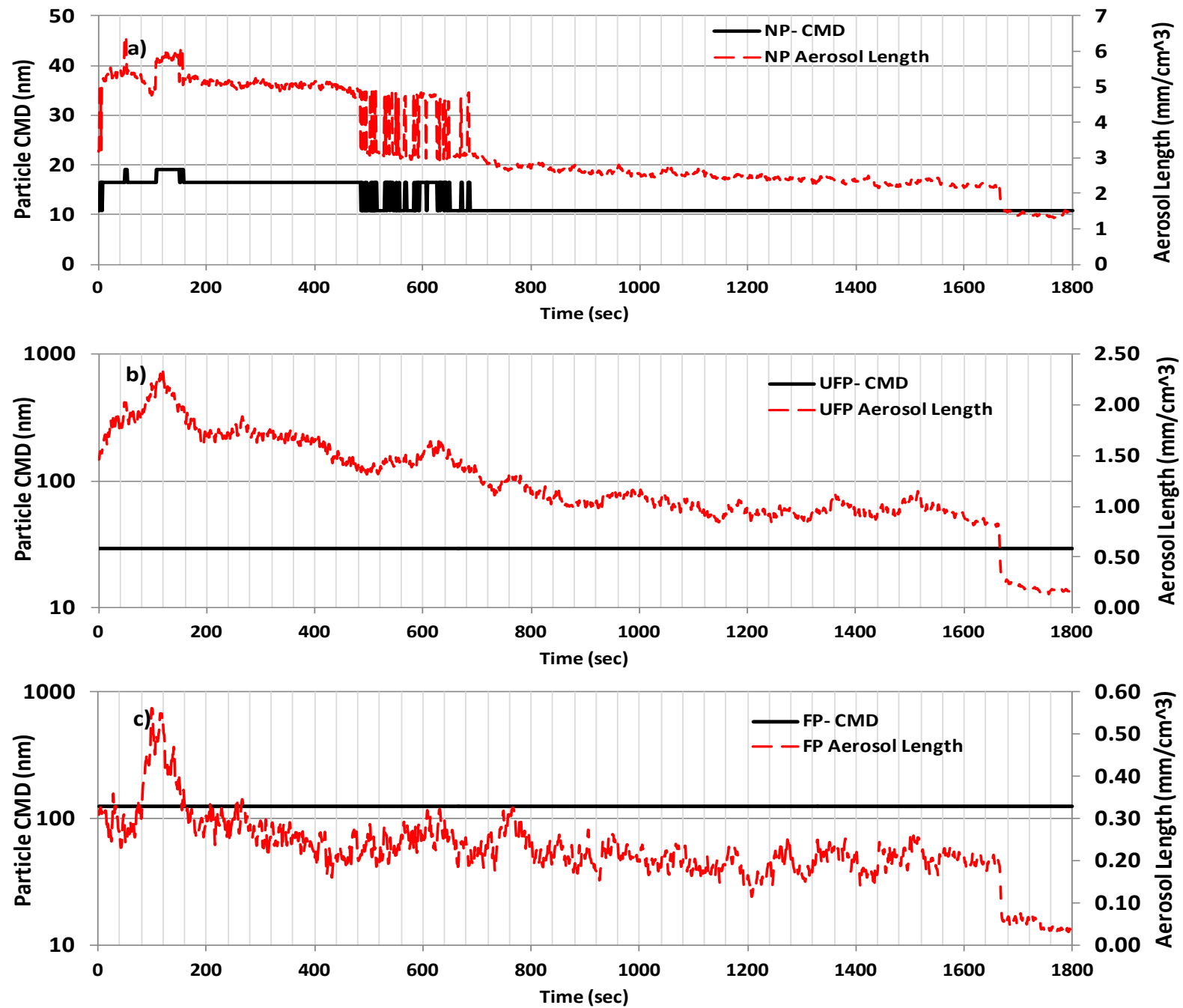

Figure 5-30 Bus 2 Idle operation a) NP bin CMD and corresponding aerosol length b) UFP bin CMD and corresponding aerosol length c) FP bin CMD and corresponding aerosol length

Figure 5-30 a) shows the results of the instantaneous CMD tracking of particle within the NP size bin with its associated aerosol length calculations. The results show a average particle 
CMD of $12 \mathrm{~nm}$ through the entire idle operation with a average aerosol length of $3.2 \mathrm{~mm} / \mathrm{cm}^{3}$. A mono-disperse aerosol stream of $12 \mathrm{~nm}$ with an aerosol length of $3.2 \mathrm{~mm} / \mathrm{cm}^{3}$ will correspond to a particle concentration of $2.67 \times 10^{5} \mathrm{\#} / \mathrm{cm}^{3}$. Hence the measured concentration is an order of magnitude greater than typical background noise, and hence can be considered as a significant tailpipe PM emission. An analysis of the instantaneous CMD tracking reveals the first 500 seconds of the operation to be characterized with higher particle concentration and slightly larger particles with CMD of $16 \mathrm{~nm}$ in the NP size bin. Beyond 600 seconds of the operation resulted in a steep decrease in aerosol length from about $5 \mathrm{~mm} / \mathrm{cm}^{3}$ to about 2.5 $\mathrm{mm} / \mathrm{cm}^{3}$, suggesting a possible change in some engine parameter that resulted in lower particle concentration.

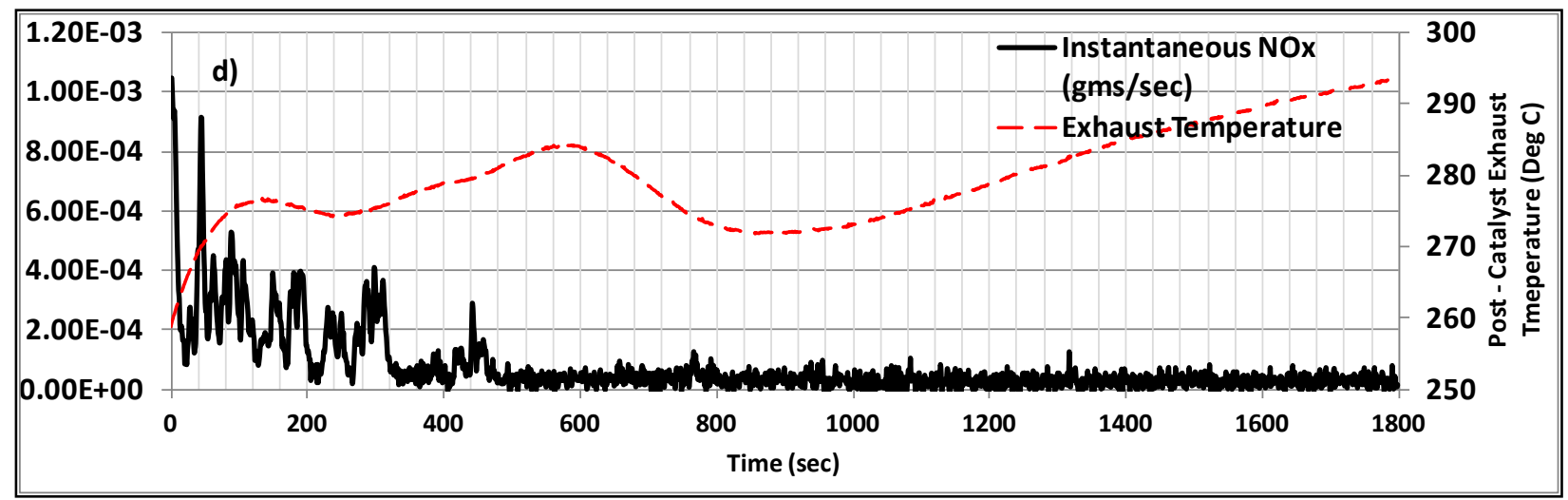

Figure 5-31 Instantaneous NOx and exhaust temperature trace during idle operation of Bus 2

Figure 5-31 shows the instantaneous NOx and exhaust temperature trace during the idle operation of bus 2. It can be seen that during the first 500 seconds of the idle period that was characterized by larger particles with higher concentration in the NP size bin, the exhaust temperatures were comparatively lower (in the range of 275 Deg C) than the later portion of the idle operation. Another interesting trend to be noted here is the subsequent decrease in NOx emissions with a gradual increase in exhaust temperature. This could be attributed to the dithering strategy of the air-fuel ratio employed in a stoichiometric engine to optimize NOx reduction over the three-way catalyst. The increase in exhaust temperature with subsequent decrease in NOx emissions can be attributed to the slight shift in equivalence ratio towards the rich mixtures, to achieve maximum NOx reductions during idle operation. It is well documented that rich air-fuel conditions, exhibit higher NOx conversion ratios over three-way catalysts, 
hence manufacturers tend to shift towards slightly richer mixture ( $\Phi>1.05$ almost $100 \%$ NOx conversion efficiencies) (Defoort et al., 2003) during transient, low speed and idle operations. This shift in air fuel ratio can have indirect implications on particle formation mechanism due to the increase in exhaust temperature and as result better catalytic activity. The $16 \mathrm{~nm}$ CMD over the initial 500 seconds operation could gas phase compound condensing on solid fractions such as metallic nanoparticles. As observed in results from bus 1, lubrication oil could contribute significantly to solid fraction emissions in the NP size bin. Assuming, we observe the same trend with bus 2 also, a combination lower exhaust temperatures and presence of low vapor phase organics (possibly lube oil based) could result in particle formation mechanism that involves heterogeneous nucleation of gas phase precursors onto solid fractions, thereby resulting in particle CMD slightly larger than 8-10 $\mathrm{nm}$ range that has been previously observed from both the vehicles. Moreover, the instantaneous change in particle CMD from $17 \mathrm{~nm}$ to $10 \mathrm{~nm}$ with increase in exhaust temperatures also suggests the possibility of the attainment of catalytic light-off temperature and the subsequent prevention of heterogeneous nucleation.

Figure 5-30 b) shows the instantaneous CMD tracking for particles within the UFP size bin with its associated aerosol length calculations. The results show a constant particle CMD of 29 $\mathrm{nm}$ for the entire idle period. The particle size distribution observed during idle operation did not result in three distinct modes however, it should evidence of tri-modal distribution within the TSI EEPS size range of $6.04 \mathrm{~nm}$ to $500 \mathrm{~nm}$. The second modal distribution was observed between the size range of $25 \mathrm{~nm}$ and $45 \mathrm{~nm}$ with the resulting CMD consistently at $29 \mathrm{~nm}$. The average aerosol length for this size bin was calculated to be $3.2 \mathrm{~mm} / \mathrm{cm}^{3}$. As illustrated the aerosol length also show a gradual decrease in its value indicating a decrease particle concentration since the CMD remained constant. It is also seen that close to 30 minutes of idle operation the particle concentration in this size range falls to levels below the detection levels of the instrument. Since, the visual inspection of the gravimetric filter suggests significant black carbon emissions, it necessary to correlate masses of the different size bins to EC emissions to discern the size bin of the particle size distribution that contributes to EC emissions.

Figure 5-30 c) shows the instantaneous CMD tracking of particles within the FP size bin with its associated aerosol length calculations. The results show a constant particle CMD of 
$124.1 \mathrm{~nm}$ through the entire duration of the idle operation with an average aerosol length of $0.22 \mathrm{~mm} / \mathrm{cm}^{3}$. This size range is commonly attributed to solid fraction of PM emissions that contribute majorly towards the mass fraction of PM. In analysis of results from bus 1 , the results indicated a possibility of tunnel artifacts to contribute to particles within this size bin. However, results from idle operation of bus 2 could suggest evidence of extensive lubrication oil combustion that contributes to a steady concentration of particles within this size bin as observed in all size bins for this vehicle operation.

Figure 5-32 shows the results of the integrated particle size distribution and concentration measurement from bus2 during the steady state $45 \mathrm{MPH}$ operation.

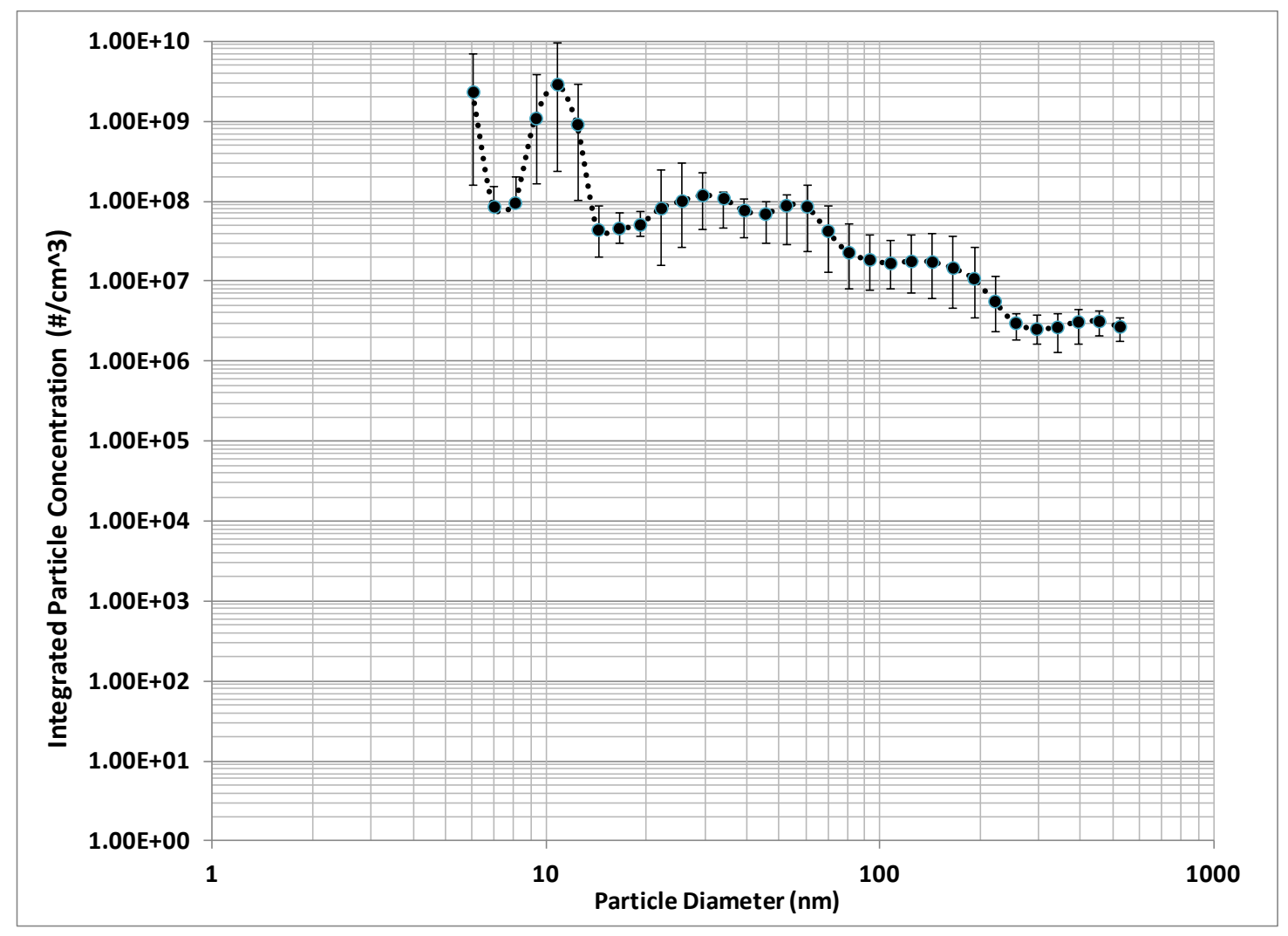

Figure 5-32 Results of integrated particle size distribution and concentration over steady state $45 \mathrm{MPH}$ operation of bus 2

As illustrated the characteristic nucleation mode peak with a CMD of $10.8 \mathrm{~nm}$ is observed during the steady state $45 \mathrm{MPH}$ operation also. However, the larger particles diameters observed during the idle operation are not very distinctive during the steady state operation, suggesting the larger particle diameters to be characteristic of idle mode operation and not 
significant during high speed vehicle operation. The observed $10.8 \mathrm{~nm}$ nucleation mode peaking at an average particle concentration of $2.92 \times 10^{9} \# / \mathrm{cm}^{3}$, appears to be a characteristic distribution of CNG vehicles with both vehicles regardless of the operation exhibiting this distribution.
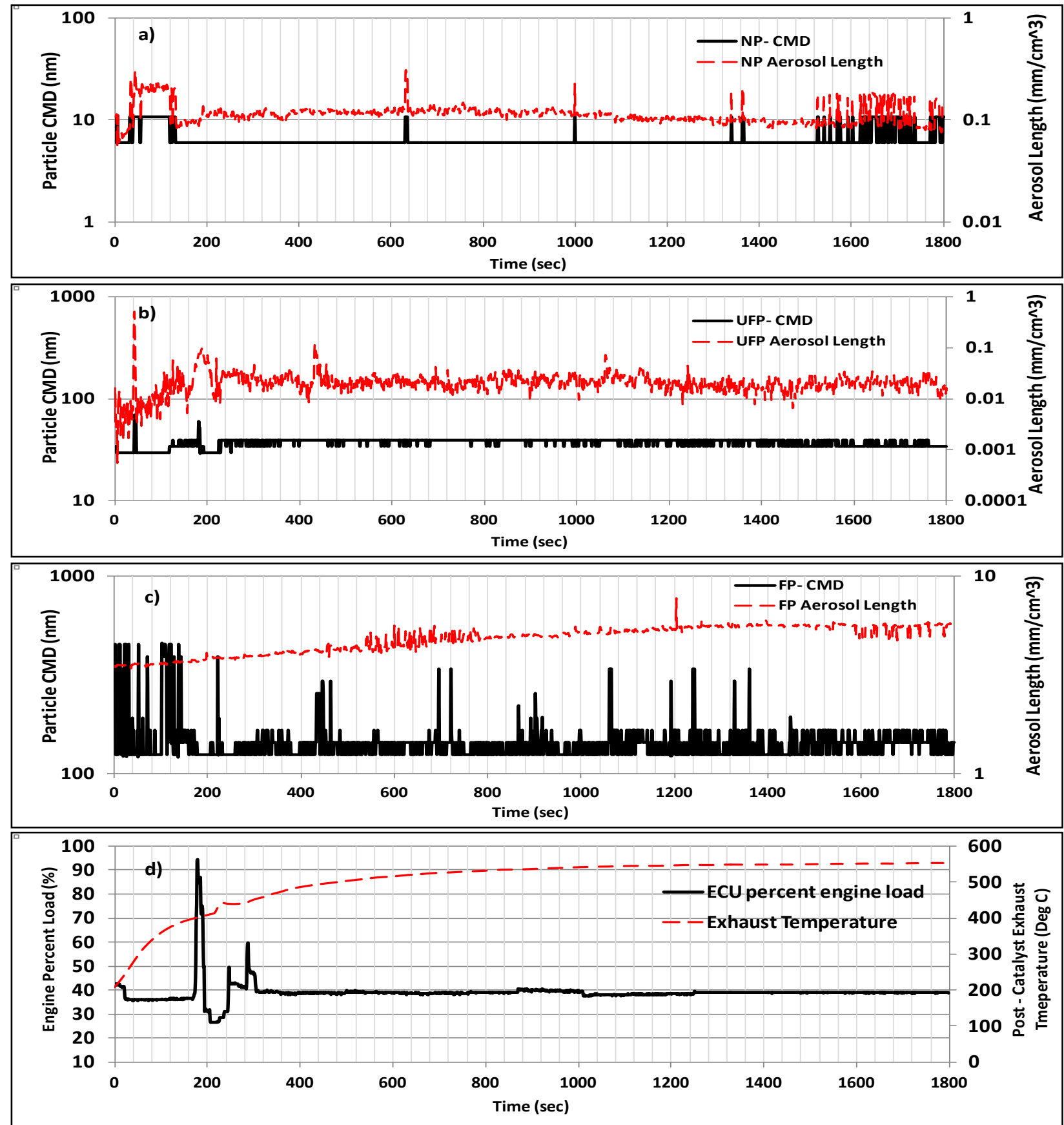

Figure 5-33 Bus 2 steady state 45 MPH operation a) NP bin CMD and corresponding aerosol length b) UFP bin CMD and corresponding aerosol length c) FP bin CMD and corresponding aerosol length d) ECU engine percent load and exhaust temperature trace 
Figure 5-33 a) shows the instantaneous particle CMD tracking of particles within the NP size bin for bus 2 during the steady state $45 \mathrm{MPH}$ operation. The results show an average CMD of $6.5 \mathrm{~nm}$ during the steady state operation with a calculated aerosol length of $0.11 \mathrm{~mm} / \mathrm{cm}^{3}$. Particle concentrations detected in this size range was close to the detection limits of the instrument, and could be characterized with higher levels of electrometer noise. However, a typical nucleation mode peak with previously observed CMD was detected during the steady state operation of this vehicle also.

Figure 5-33 b) shows the instantaneous CMD tracking of particles within the UFP size bin for bu2 during the steady state operation. The results showed an average CMD of $37 \mathrm{~nm}$ with a

corresponding average aerosol length of $0.02 \mathrm{~mm} / \mathrm{cm}^{3}$. The calculated aerosol lengths over the entire duration of the steady state cycle indicate particle concentrations below the detection limits of the instrument. The EC/OC analysis from bus 2 over the $45 \mathrm{MPH}$ cycle reported no EC mass emissions, and the particle concentrations within the UFP size bin also, corroborate the findings of the EC/OC analysis.

Figure 5-33 c) shows the instantaneous tracking of particle CMD within the FP size bin, with its associated aerosol length calculation. The particle CMD within the size range showed high variation with an average CMD value of $145 \mathrm{~nm}$. The aerosol length calculations for the observed CMD range indicate particles with particle count that could be associated with high levels of electrometer noise. Gravimetric results of PM from bus 2 indicated the lowest PM mass emissions compared to the idle and transient operating conditions. The particle size distribution analysis, together with the size bin based analysis indicate particle concentrations close to the detection limits of the instrument, with no significant particle contribution from any of the three particle size bins.

\subsection{RESULTS OF PARTICLE MASS ANALYSIS}

This section presents the results of the mass calculations derived from the particle size distribution and concentration measurement performed for the two buses. This section will sue the effective density functions explained in detail in section 2.5.2. The particle mass results presented by the post processing tool of the TSI EEPS assumes unit density, while the results 
presented in this section will use the density function developed by Maricq et al. Since, the gravimetric filter sampling represents all a PM particles below the cut point of $2.5 \mu \mathrm{m}$, the section will use the custom defined bins of nanoparticle (NP) of size range between 6.04 and $25.5 \mathrm{~nm}$, ultra-fine particle (UFP) of size range between 29.4 and $107 \mathrm{~nm}$ and fine particle (FP) with size range between 124 and $523.3 \mathrm{~nm}$. The bin wise calculation of particle mass will further enable to present a correlation between mass of various exhaust species and their respective category in the total particle size distribution.

Table 20 lists the distance specific and time specific (idle operation) PM mass emissions calculated from particle size distribution and concentration measurements of bus 1 . The computed values for the UDDS cycle show an average $7.08 \mu \mathrm{g} / \mathrm{mi}$ mass emissions of PM within the NP size bin, $0.155 \mathrm{mg} / \mathrm{mi}$ and $1.415 \mathrm{mg} / \mathrm{mi}$ mass emissions within the UFP and FP size bin respectively. The results show that the mass calculated for the NP size bin, exhibit greater repeatability with a standard deviation $2.60 \mu \mathrm{g} / \mathrm{mi}$ in comparison to standard deviation of 130.36 and $1173.27 \mathrm{\mu g} / \mathrm{mi}$ for the UFP and FP size bin respectively. The higher variability of mass for the UFP and FP size bin is directly related to the high run to run variation of particle size distribution and concentration measurements reported in this size range.

Particulate matter mass emissions in the NP size bin for the steady state $45 \mathrm{MPH}$ operation was similar to the UDDS operation with an average distance specific mass of $2.562 \mu \mathrm{g} / \mathrm{mi}$. The NP size bin reported particle mass emissions with low standard deviation.

Table 20 Results of the bin wise particle mass calculations of bus 1

\begin{tabular}{cccc}
\hline & Nanoparticle $(\mu \mathrm{g} / \mathrm{mi})$ & $\begin{array}{c}\text { Ultrafine Particle } \\
(\mathrm{mg} / \mathrm{mi})\end{array}$ & $\begin{array}{c}\text { Fine Particle } \\
(\mathrm{mg} / \mathrm{mi})\end{array}$ \\
\cline { 2 - 4 } UDDS & 9.364 & 0.3222 & 1.971 \\
& 5.846 & 0.0676 & 0.749 \\
& 4.024 & 0.0369 & 0.171 \\
Average & 9.117 & 0.1929 & 2.768 \\
Max & 7.088 & 0.155 & 1.415 \\
Min & 9.364 & 0.322 & 2.768 \\
Std Dev & 3.064 & 0.118 & 1.244 \\
( $\mu$ g/mile) & & & 1173.27 \\
\hline SS 45 MPH & 2.60 & 130.36 & Fanoparticle $(\mu \mathrm{g} / \mathrm{mi})$ \\
\hline
\end{tabular}




\begin{tabular}{|c|c|c|c|}
\hline & & (mg/mi) & (mg/mi) \\
\hline & 2.414 & 0.1167 & 0.9369 \\
\hline & 0.349 & 0.0024 & 0.0093 \\
\hline & 0.055 & 0.0005 & 0.0023 \\
\hline & 0.113 & 7.873E-06 & $2.782 \mathrm{E}-05$ \\
\hline & 9.878 & 0.4057 & 2.0232 \\
\hline Average & 2.562 & 0.105 & 0.594 \\
\hline Max & 9.878 & 0.406 & 2.023 \\
\hline Min & 2.507 & 0.105 & 0.594 \\
\hline $\begin{array}{c}\text { Std Dev } \\
(\mu \mathrm{g} / \mathrm{mile})\end{array}$ & 4.21 & 175.38 & 895.12 \\
\hline \multirow{4}{*}{ Idle (mass/hr) } & Nanoparticle ( $\mu \mathrm{g} / \mathrm{hr})$ & Ultrafine Particle (mg/hr) & $\begin{array}{c}\text { Fine Particle } \\
(\mathrm{mg} / \mathrm{hr})\end{array}$ \\
\hline & 0.563 & $4.152 \mathrm{E}-03$ & 1.627E-03 \\
\hline & 3.535 & 2.327E-06 & $1.200 \mathrm{E}-06$ \\
\hline & 4.618 & $1.166 \mathrm{E}-04$ & $9.103 \mathrm{E}-04$ \\
\hline Average & 2.905 & 0.001 & 0.001 \\
\hline Max & $1.713 \mathrm{E}+00$ & $2.728 \mathrm{E}-03$ & 7.810E-04 \\
\hline Min & $2.342 \mathrm{E}+00$ & $1.421 \mathrm{E}-03$ & $8.451 \mathrm{E}-04$ \\
\hline Std Dev $(\mu \mathrm{g} / \mathrm{hr})$ & 2.10 & 2.36 & 0.81 \\
\hline
\end{tabular}

The NP size bin mass PM mass emission during the idle operation was found to be 2.90 $\mu \mathrm{g} / \mathrm{hr}$. Mass emissions in the NP size bin was two orders of magnitude lower than that observed during the UDDS and steady-state $45 \mathrm{MPH}$ operation.

Figure 5-34 shows the graphical representation of data presented in Table 20. The error bars in the chart represent maximum and minimum deviations from the calculated average PM mass emissions. The results challenge existing outlook of heavy-duty natural gas PM emissions, which considers lower exhaust temperature activity such as low speed and idle operation to contribute to the bulk of the nucleation mode particle mass and number concentrations. Insufficient catalytic activity has been attributed to the bulk of the nanoparticle formation due to higher tailpipe concentration nucleation mode precursors. However, advanced stoichiometric natural gas engines are not plagued by lower exhaust temperatures, and hence exhibit sufficient catalytic activity of low vapor pressure higher chain hydrocarbons, even at idle operating conditions. Hence, the nucleation mode particles observed during almost all vehicle 
operations with varying degrees of magnitude seem to indicate a particle formation mechanism that is independent of catalytic activity or in other words a particle composition not representing particle formation mechanism of gas to particle conversion.

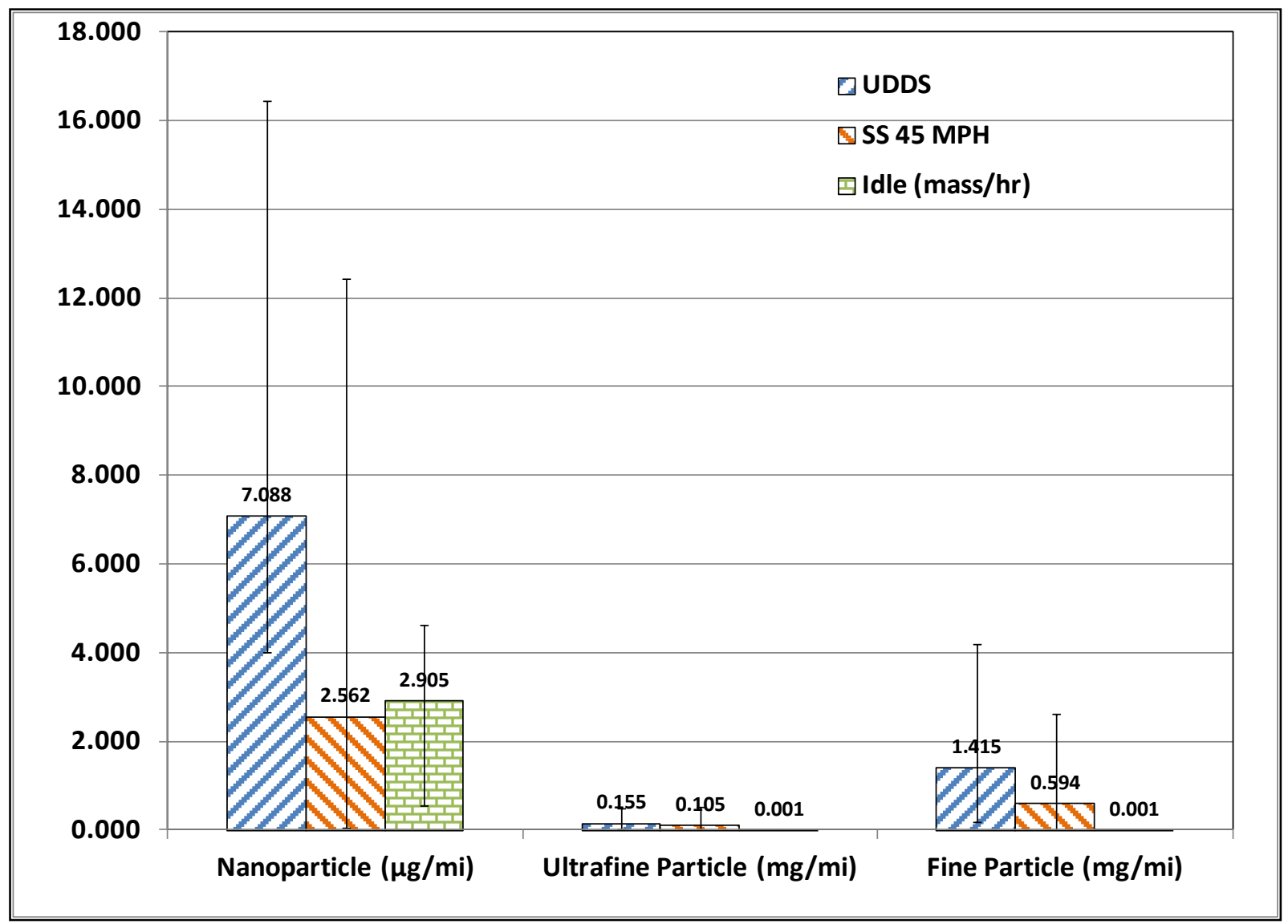

Figure 5-34 Bar plot of bin wise particle mass calculations of bus 1

Table 21 shows the results of the bin wise particle mass calculations for bus 2 over the three vehicle operation. Distance specific PM mass emissions over the UDDS cycle for bus 2 is comparable to that observed in bus 1 . The average NP size bin PM mass for the UDDS cycle was calculated to be $6.95 \mu \mathrm{g} / \mathrm{mi}$, with a standard deviation of $4.69 \mu \mathrm{g} / \mathrm{mi}$. The average mass emissions in the NP size bin between the two vehicles are within $2 \%$ of each other, suggesting a similar particle formation mechanism between both vehicles over a transient operation. 
Table 21 Results of the bin wise particle mass calculations of bus 2

\begin{tabular}{|c|c|c|c|}
\hline \multirow{5}{*}{ UDDS } & Nanoparticle ( $\mu \mathrm{g} / \mathrm{mi})$ & $\begin{array}{l}\text { Ultrafine Particle } \\
\text { (mg/mi) }\end{array}$ & $\begin{array}{l}\text { Fine Particle } \\
(\mathrm{mg} / \mathrm{mi})\end{array}$ \\
\hline & 13.531 & 0.7539 & 2.742 \\
\hline & 7.066 & 0.3168 & 4.189 \\
\hline & 4.050 & 0.2768 & 0.389 \\
\hline & 3.157 & 0.1643 & 0.196 \\
\hline Average & 6.951 & 0.378 & 1.879 \\
\hline Max & 13.531 & 0.754 & 4.189 \\
\hline Min & 3.794 & 0.214 & 1.683 \\
\hline $\begin{array}{l}\text { Std Dev } \\
(\mu \mathrm{g} / \mathrm{mile})\end{array}$ & 4.69 & 258.80 & 1926.49 \\
\hline \multirow{5}{*}{ SS $45 \mathrm{MPH}$} & Nanoparticle $(\mu \mathrm{g} / \mathrm{mi})$ & $\begin{array}{c}\text { Ultrafine Particle } \\
(\mathrm{mg} / \mathrm{mi})\end{array}$ & $\begin{array}{c}\text { Fine Particle } \\
(\mathrm{mg} / \mathrm{mi})\end{array}$ \\
\hline & 0.494 & 0.010 & 0.030 \\
\hline & 0.207 & 0.005 & 0.017 \\
\hline & 0.185 & 0.003 & 0.014 \\
\hline & 1.395 & 0.005 & 0.020 \\
\hline \multirow{4}{*}{$\begin{array}{c}\text { Average } \\
\text { Max } \\
\text { Min } \\
\text { Std Dev } \\
\text { ( } \mu \mathrm{g} / \mathrm{mile})\end{array}$} & 0.571 & 0.006 & 0.020 \\
\hline & 1.395 & 0.010 & 0.030 \\
\hline & 0.385 & 0.002 & 0.006 \\
\hline & 0.57 & 2.67 & 6.85 \\
\hline \multirow{5}{*}{ Idle (mass/hr) } & Nanoparticle $(\mu \mathrm{g} / \mathrm{hr})$ & Ultrafine Particle (mg/hr) & $\begin{array}{l}\text { Fine Particle } \\
\text { ( } \mathrm{mg} / \mathrm{hr})\end{array}$ \\
\hline & 1948.37 & 9.86 & 7.87 \\
\hline & 1073.04 & 3.38 & 2.50 \\
\hline & 2650.13 & 19.27 & 13.33 \\
\hline & 1748.60 & 4.74 & 3.30 \\
\hline Average & 1855.036 & 9.311 & 6.750 \\
\hline Max & 795.10 & 9.96 & 6.58 \\
\hline Min & 781.99 & 5.93 & 4.25 \\
\hline Std Dev $(\mu \mathrm{g} / \mathrm{hr})$ & 649.03 & 7201.63 & 4984.78 \\
\hline
\end{tabular}

The average NP size bin mass for the steady state operation for bus 2 resulted in an order of magnitude lower particle mass in comparison to bus 1 . The NP size bin mass for the steady state operation from bus 2 was calculated to be $0.571 \mu \mathrm{g} / \mathrm{mi}$ with a standard deviation of 0.57 $\mu \mathrm{g} / \mathrm{mi}$. If lubrication oil is assumed to contribute to the bulk of the NP size bin mass, then bus 2 steady state operation resulted in lower lubrication consumption in comparison to bus 1. 
One of the interesting findings of the study is the observed mass emissions rate of PM from bus 2 during its idle operation. PM mass emission in the NP size bin during the idle operation was found to be three orders of magnitude greater than that observed in bus 1 . The average mass emission in the NP size bin was calculated to be $1855.03 \mu \mathrm{g} / \mathrm{mi}$ with a standard deviation of $649.03 \mu \mathrm{g} / \mathrm{mi}$. Similarly, the UFP and FP size bin also reported mass that are 3 orders of magnitude greater than that observed from bus 1.

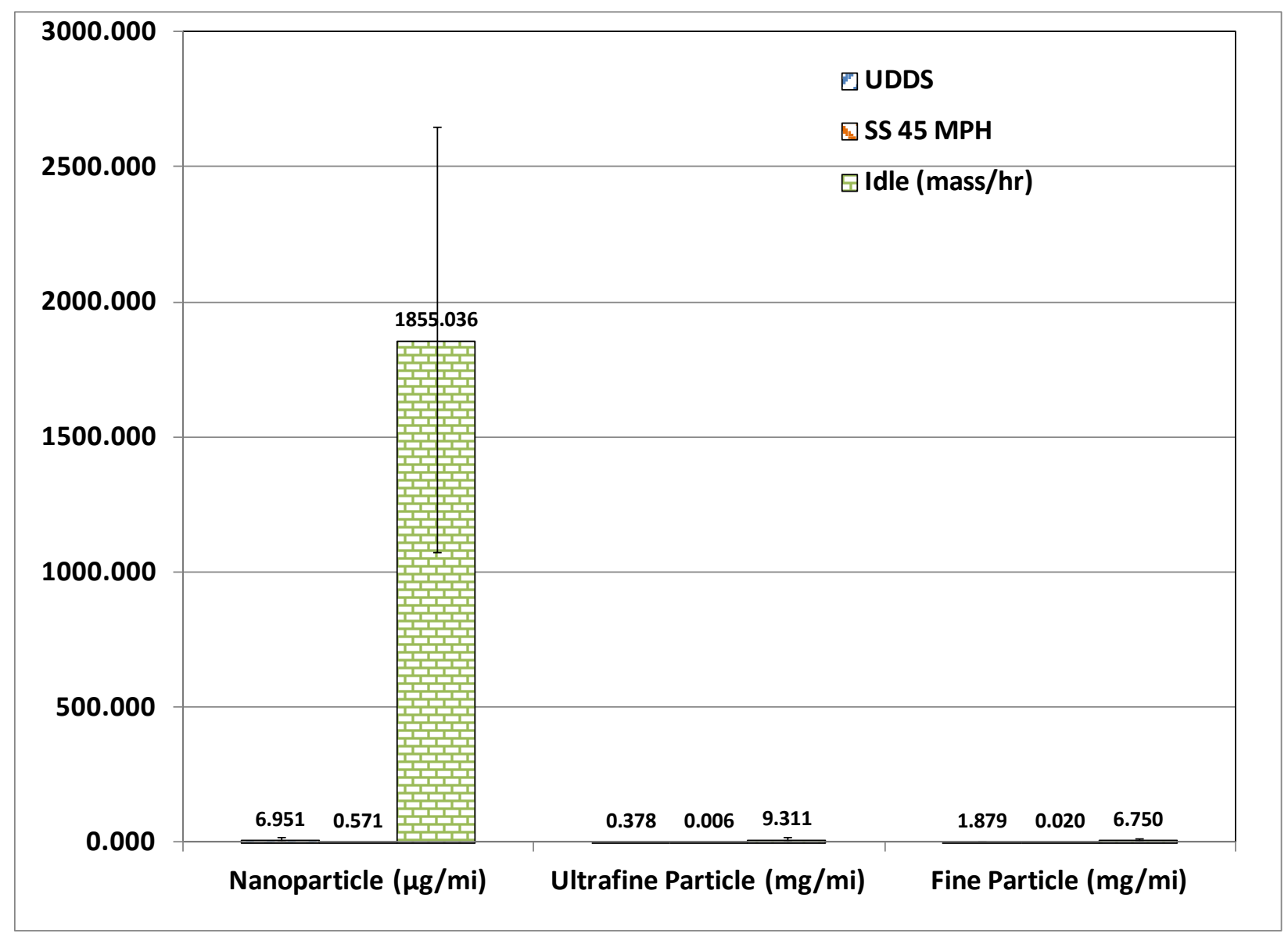

Figure 5-35 Bar plot of bin wise particle mass calculations of bus 2

Figure 5-35 shows the graphical representation of the bin wise mass calculation results of bus 2 shown in Table 21. The results show evidence of extensive lubrication oil consumption that has manifested itself as particle mass emissions in all three size bins namely NP, UFP and FP. This further suggest the possibility of oil entry into the combustion chamber resulting in ash and EC emissions contributing to mass in the UFP and FP size bin. However, the lack of volatile precursors and no evidence of homogenous nucleation presents a case of uncertainty in 
attempting discern the composition of the NP size bin. The upcoming sections will present correlation of mass within this size bin with individual speciated mass in order to suggest possible compositional scenario for PM in this size bin.

\subsubsection{PARTICLE MASS ANALYSIS CORRELATIONS}

The previous sub sections of the results chapter, elucidated the mass correlations of various fractions of PM namely, metals, EC/OC and certain PAH compounds to toxicity responses. The results indicated a strong correlation of mass of metals to both DTT and the alveolar macrophage assay. Since, a size segregated particle mass collection was not employed this section will attempt to correlate particle mass in the defined bins with mass of different PM fractions to infer the composition of the different size bins of the particle size distribution.

Table 22 shows the results of the Pearson's correlation analysis performed between mass of particles within the NP size bin and mass of metal emissions. The analysis considered mass of only 4 elements namely zinc, phosphorus, chromium and iron, since these elements exhibited statistically significant correlation towards response from DTT and the alveolar macrophage assay. Also, the four selected elements are characteristic components of lubrication oil consumption, and were found to comprise a significant fraction of the total elemental emissions

Table 22 Results of Pearson correlation coefficient and level of significance between elemental emissions and bin wise PM mass emissions

\begin{tabular}{ccc}
\hline \multicolumn{3}{c}{ Metals (Zn, P, Cr, Fe) } \\
\hline Size Bin & $\mathrm{R}$ & $\mathrm{P}$ \\
\hline NP & 0.96 & 0.002 \\
UFP & 0.25 & 0.636 \\
FP & -0.09 & 0.864 \\
\hline
\end{tabular}

The results exhibited an interesting correlation between the particle mass in the NP size bin with the elemental emissions. A Pearson's correlation factor of 0.96 with a level of significance p $=0.002$ was obtained. Hence, a statistically significant correlation in the $99 \%$ confidence interval is reported for the correlation of the NP size bin mass and metallic elements. It is to be noted that the results presented here is based on a statistical correlation only, since a size 
based PM sampling or results from a scanning electron microscope (SEM) is not available to corroborate the statistical significance.

Despite the lack of experimental evidence to corroborate the finding, an important study in the literature has documented a similar finding that suggest the possibility of nanoparticles composed of metallic and elemental fractions. The study by Lee et al. was aimed at gaining insight into the fraction of metal emissions in the ultrafine particle portion. To accomplish this goal the investigators used a $1.9 \mathrm{~L}$ diesel generator, with fuel doped with varying levels of iron crystals. The results of the study showed an interesting particle size distribution that indicated self nucleation of the iron fraction in the fuel after combustion to form nanoparticles in the size range of $10 \mathrm{~nm}$. Further, the study indicated that engine load conditions with lower carbon content, enhanced the concentration of this nucleation mode, suggested to be made up of iron nanoparticles. The study further corroborated its finding through SEM images on these nanoparticles with single particle mass spectroscopy that confirmed the iron content. Also, one of the important conclusions of the study indicated the possibility of enhanced self nucleation of metallic particles during conditions of low soot or ultra low level soot emissions (Lee et al., 2006).

Figure 5-36 shows the particle size distribution results from the study by Lee et al. (Lee et al., 2006). The nucleation mode shown in the referenced study is very similar to the nucleation mode observed in the current study, and further corroborated by statistically significant correlation exhibited by nanoparticle size bin and elemental emissions. 


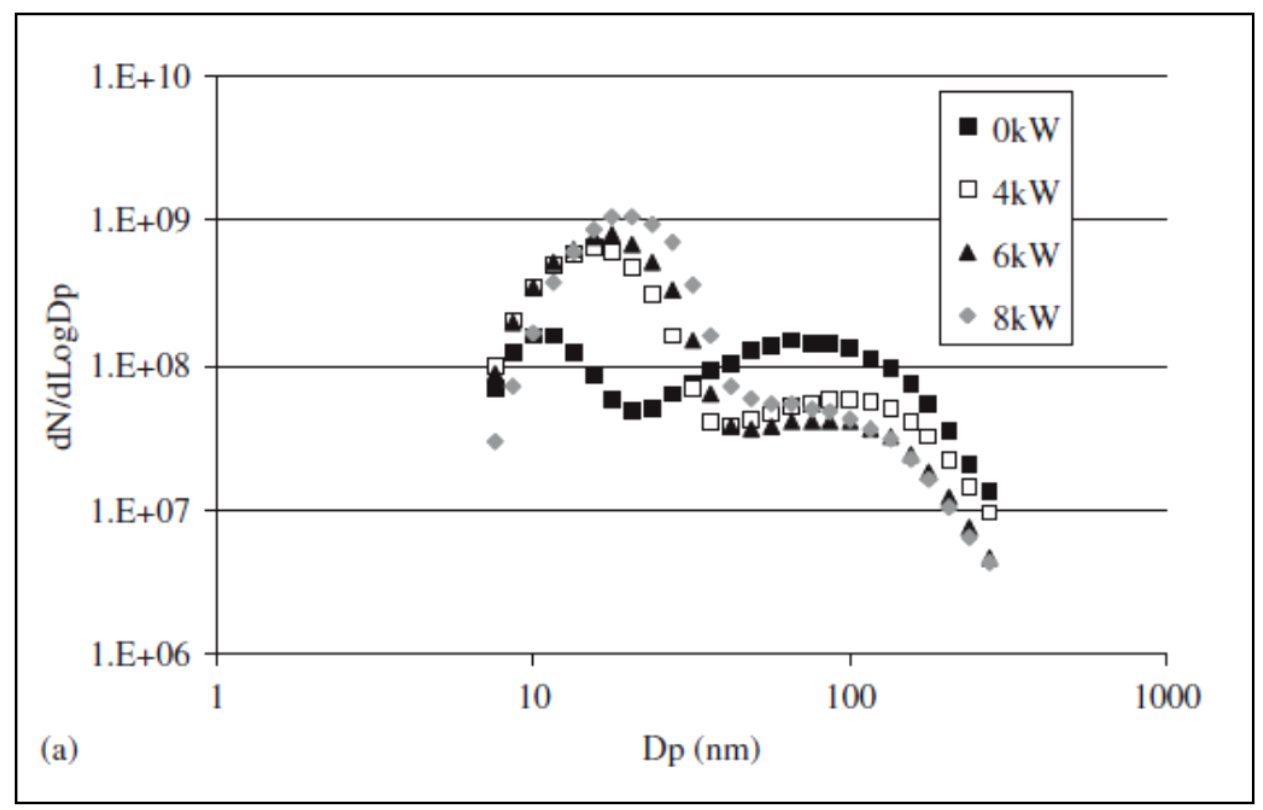

Figure 5-36 Chart adapted from (Lee et al., 2006) showing the iron based nucleation mode for a diesel fuel doped with $60 \mathrm{ppm}$ iron.

A comprehensive study by Khalek et al. has shown that lubrication oil additives undergo volatilization when passing thorugh the combustion chamber and a fraction of them renucleate to form nanoparticles while the remaining adsorb on to other accumulation mode particles. The study also documents that low soot conditions are most favorable conditions for nucleation of lubrication oil derived metals and elements. The study by Khalek et al. also suggests at the possibility of inorganic components such as calcium, zinc and magnesium forming solid nanoparticles (khalek et al., 1998).

Although, the above referenced study has pointed out the possibility of a low soot engine favoring, nucleation of inorganic species in the exhaust, no previous study has reported this finding. The salient feature of the current study is the fact that the lack of sulfur in the fuel, and the gaseous nature of the fuel, helps investigate the composition of the observed nucleation mode without any interference from possible sulfate formation, sulfuric acid particles and high EC content in the exhaust.

Although, lubrication oil based sulfur has been reported to be a significant contributor to formation of sulfuric acid particles during high temperature exhaust conditions (Thiruvengadam et al., 2012, Kittelson et al., 2008, Vaaraslahti et al., 2005), the sulfate composition in the 
exhaust of the natural gas engines have been found close to the levels observed in the background. Moreover, sulfur was observed in abundance as elemental composition rather than as a sulfate fraction as expected due to the predominant high temperature operation of stoichiometric natural gas engines. This is due to the fact that sulfate based nanoparticles are formed due to successive oxidation sulfur to $\mathrm{SO}_{2}$ in the combustion chamber and further to $\mathrm{SO}_{3}$ over catalytic surfaces. However, it is to be noted that $\mathrm{SO}_{2}$ to $\mathrm{SO}_{3}$ conversion can be achieved only with excess oxygen in the exhaust and at temperatures greater than $380 \mathrm{deg} C$. In the case of stoichiometric natural gas engines, lubrication oil based sulfur can still under go in cylinder oxidation to $\mathrm{SO}_{2}$, however a successive oxidation to $\mathrm{SO}_{3}$ would not be possible due to the oxygen deprived exhaust gas composition that is characteristic of stoichiometric engine operation. The lack of $\mathrm{SO}_{3}$ content inhibits the binary nucleation of sulfuric acid in the presence of water vapor. Hence, the nucleation mode observed in the current study differs from the nucleation mode observed from high temperature operation of advanced after-treatment equipped diesel engines, with respect to its composition. Nucleation mode observed from DPFSCR equipped vehicles are believed to be a result of binary nucleation of $\mathrm{SO}_{3}$ and water forming sulfuric acid droplets and that formed in the exhaust of stoichiometric natural gas engines seem to indicate a solid nucleation mode particles composed of inorganic fraction such as metals and elements.

The exhaust of the stoichiometric natural gas engine tested in this study does not exhibit significant mass emissions of sulfates and elemental carbon, but a significant mass emissions of lubrication oil based elements and metals such as phosphorus, zinc, magnesium, calcium, chromium and iron is observed together with a particle size distribution that is completely dominated in the nucleation mode size bin. The high temperature three-way catalyst activity also resulted in VOC and PAH content close to detection limits of the analytical method. Hence, the collective evidence, suggests a nucleation mode particle formation mechanism that represents a re-nucleation of combusted lubrication oil inorganic components during exhaust gas cooling and dilution.

Table 23 shows the Pearson's correlation results for mass of particles in the NP size bin with organic carbon mass and responses from DTT and alveolar macrophage assays. The 
correlation of organic carbon and NP size bin mass resulted in a fairly good correlation, but with no statistical significance. This could be due to the fact that the OC mass measured through the NIOSH method with a pre-fired quartz filter could be characterized by higher levels of positive artifacts from the high methane content in the natural gas engine exhaust, together with the affinity of pre-fired quartz in adsorbing hydrocarbons. Also, to corroborate the finding that indicates a solid inorganic nucleation mode fraction a correlation of NP size bin with DTT and alveolar macrophage ROS was performed. The results indicate good correlation factors of 0.98 and 0.76 for DTT and macrophage ROS respectively. The DTT correlations indicate a statistically significant correlation factor within the 95\% confidence interval, and the macrophage ROS resulting in a correlation factor marginally outside the $95 \%$ confidence interval, but can still be considered as statistically significant. Since, the DTT and the ROS macrophage assays independently correlated well with the mass of phosphorus, zinc, chromium and iron it lends support to the possibility a NP size bin consisting of inorganic self nucleated particles.

Table 23 Results of Pearson correlation coefficient and level of significance NP mass and DTT and ROS responses

\begin{tabular}{ccc}
\hline \multicolumn{3}{c}{ NP Size Bin } \\
\hline Size Bin & R & $P$ \\
\hline OC & 0.62 & 0.18 \\
DTT & 0.98 & 0.02 \\
ROS & 0.76 & 0.08 \\
\hline
\end{tabular}

It is clear from many early research papers that certain elemental emissions such as zinc, phosphorus, calcium and magnesium are characteristic of lubrication oil contribution. Most of these elements are major additives in lubrication oil that impart various properties to the oil in terms of rust protection, maintain the acidity of the oil, wear protection and viscosity enhancers. Additive such as zinc dialkyldithiophosphates (ZDDP) developed by Castrol is commonly used to impart antioxidant and anti-wear properties to lubrication oil. A study by Hosonuma et al. has elucidated the decomposition of ZDDP in an engine and its interaction with diesel soot. The study reports that ZDDP decomposes into zinc free phosphorus compounds and phosphorous free zinc compounds in an engine. And further only the free zinc is capable of combining with soot as an agglomerate and not phosphorus (Hosonuma et al., 1985). In relation to the current study, the results might be indicative of a similar decomposition of 
possible ZDDP additives in the lubrication oil that manifests itself as free phosphorus and zinc fractions in the exhaust, and further forming particles in the NP size bin.

The path of the lubrication oil into the combustion chamber or directly in to the exhaust needs to be addressed while attempting to link lubrication oil consumption with PM emissions. The magnitudes of lubrication oil based elemental emissions seem to suggest a significant contribution from lubrication oil independent of vehicle operation. Further, the presence of nucleation mode with a particle concentration of same order of magnitude between transient, idle and steady state operation indicates an oil transport mechanism not dependent on in cylinder pressure conditions. In the case of oil transport through piston rings, idle and low load operation would result higher elemental emissions and steady state operations with comparatively higher steady loads would result in lower elemental emissions. However, the results seem to indicate an oil transport mechanism such as through a closed crankcase ventilation (CCV) system which is a load independent contribution of oil into the combustion chamber. Beginning of MY 2007 engine manufacturers were mandated to either include crankcase emissions as part of the exhaust emission while certifying emissions or employ closed circuit crank case ventilation to induct crank case fumes back into the intake through the turbochargers. Crankcase blow-by gas consists of a mixture of in cylinder combustion gases together with oil in vapor and fine liquid droplets. To protect the components of the turbochargers from piqued oil droplets manufacturers usually employ filter or impaction methods to remove solid and liquid fractions of the blow by gas in order to induct only gas phase components into the combustion chamber. In sufficient maintenance of the CCV system could possibly result in loss of filtration efficiency of the blow-by gas contributing to higher levels of lubrication oil components into the combustion chamber. Since, the ISLG series Cummins engine employ CCV system with possibility of either a filter mechanism or impaction method to remove liquid and solid fractions of lubrication oil. However, the operational efficiencies of these filtration mechanisms are not monitored and could result in increased lubrication oil fractions to be inducted in to the intake of the engine. As a result we could be observing the combustion and the subsequent re-nucleation of the inorganic lubrication oil components to form nanoparticles in the exhaust. 
Another interesting result from the current study is the high mass emission of PM during the idle operation of bus 2. EC/OC analysis indicated an EC emission rate of $0.005 \mathrm{mg} / \mathrm{sec}$ during the idle operation of bus 2. The particle size distribution indicated a broad distribution with significant particle concentrations in NP, UFP and FP size bins. Since EC mass was not detected in all vehicle operations from both buses a statistical correlation was not possible. However, the sum of particle mass in the UFP and FP size bin resulted in a total mass (16.06 $\mathrm{mg} / \mathrm{hr}$ ) that is $10.7 \%$ lower than the EC mass $(18 \mathrm{mg} / \mathrm{hr})$ during the idle operation. The sum of particle masses from all the size bins resulted in a total mass of $17.91 \mathrm{mg} / \mathrm{hr}$ which is $0.47 \%$ lower than the EC mass during the same operation. The percent differences seem to indicate possible EC fractions in all size bins of the particle size distribution.

\subsubsection{SUMMARY OF ANALYSIS}

Table 24 shows the tabulated results of the different components analyzed and their statistical correlations with different toxicity assays. Symbol $\uparrow$ and $\downarrow$ in the table is used to denote the concentration of a given analyte to be high and low relative to background concentrations respectively. Symbol $\underline{1}$ is used to denote the possibility of higher concentration relative to background due to the presence of sampling artifacts such as gas phase adsorption on filter. Symbol $\uparrow$ is used to denote high variability in the measured data due to measurement close to the detection limits of the instrument. Symbol ** denotes Pearsons correlation coefficient with statistical significance level or 0.01 or in the $99 \%$ confidence interval. Symbol * denotes Pearsons correlation coefficient with statistical significance level or 0.05 or in the $95 \%$ confidence interval. $\diamond$ denotes poor Pearsons coefficient with statically insignificant $p$ value. 
Table 24 Tabulated summary of correlation and statistical significance of different components analyzed

\begin{tabular}{|c|c|c|c|c|c|}
\hline \multirow[t]{2}{*}{ Component } & \multirow[t]{2}{*}{$\begin{array}{c}\text { Concentration } \\
\text { relative to } \\
\text { Bckgnd }\end{array}$} & \multirow[t]{2}{*}{ Relationship } & \multirow[t]{2}{*}{$\begin{array}{l}\text { Level of } \\
\text { Correlation }\end{array}$} & \multicolumn{2}{|c|}{$\begin{array}{c}\text { Statistical Significance of } \\
\text { Correlation }\end{array}$} \\
\hline & & & & Significant & Insignificant \\
\hline \multirow{3}{*}{ EC } & Transient $\uparrow$ & DTT & Good & & 0 \\
\hline & Steady state $\downarrow$ & DHBA & Poor & & 0 \\
\hline & Idle $\uparrow$ & ROS & High & $* *$ & \\
\hline \multirow{3}{*}{ OC } & Transient $\mathbb{I}$ & DTT & Poor & & $\diamond$ \\
\hline & Steady state $\mathbb{1}$ & DHBA & Poor & & $\Delta$ \\
\hline & Idle 1 & ROS & Poor & & $\Delta$ \\
\hline \multirow{3}{*}{ PAH/VOC } & \multirow{3}{*}{$\begin{array}{c}\text { On all driving } \\
\text { types } \downarrow\end{array}$} & DTT & High & $\begin{array}{c}\mathrm{MW}<200 \\
* *\end{array}$ & \\
\hline & & DHBA & Poor & & $\Delta$ \\
\hline & & ROS & Poor & & $\Delta$ \\
\hline \multirow{3}{*}{ Carbonyl } & \multirow{3}{*}{$\begin{array}{c}\text { On all driving } \\
\text { types } \downarrow\end{array}$} & DTT & Poor & & $\Delta$ \\
\hline & & DHBA & Poor & & $\Delta$ \\
\hline & & ROS & Poor & & $\Delta$ \\
\hline \multirow{3}{*}{ Metals/Elements } & \multirow{3}{*}{$\begin{array}{l}\text { On all driving } \\
\text { types } \uparrow\end{array}$} & DTT & High & $\begin{array}{c}(\mathrm{Cu}, \mathrm{P}, \mathrm{Zn}) \\
\quad * *\end{array}$ & \\
\hline & & DHBA & Good & & $\begin{array}{c}(\mathrm{S}, \mathrm{K}, \mathrm{V}, \mathrm{Fe}, \\
\mathrm{Co}) \diamond\end{array}$ \\
\hline & & ROS & High & $\begin{array}{l}(\mathrm{Cu}, \mathrm{P}, \mathrm{S}, \mathrm{Cr}, \\
\mathrm{Fe}, \mathrm{Zn}) * *\end{array}$ & \\
\hline \multirow{6}{*}{$\begin{array}{l}\text { Nucleation mode } \\
\text { particles }\end{array}$} & \multirow{6}{*}{$\begin{array}{l}\text { On all driving } \\
\text { types } \uparrow\end{array}$} & $\begin{array}{l}\text { Metals/ } \\
\text { elements }\end{array}$ & High & $\begin{array}{l}(\mathrm{P}, \mathrm{Cr}, \mathrm{Fe} \\
\mathrm{Zn})\end{array}$ & \\
\hline & & EC & Poor & & $\Delta$ \\
\hline & & OC & Poor & & 0 \\
\hline & & DTT & High & $* *$ & \\
\hline & & ROS & Good & $*$ & \\
\hline & & PAH & Poor & & $\diamond$ \\
\hline \multirow{4}{*}{$\begin{array}{c}\text { Accumulation mode } \\
\text { particles }\end{array}$} & \multirow{4}{*}{$\begin{array}{l}\text { On all driving } \\
\text { types } \uparrow\end{array}$} & $\begin{array}{l}\text { Metals/ } \\
\text { elements }\end{array}$ & Poor & & $\diamond$ \\
\hline & & EC & Good & & $\Delta$ \\
\hline & & OC & Poor & & $\diamond$ \\
\hline & & PAH & Poor & & $\diamond$ \\
\hline
\end{tabular}




\subsection{REGIONAL DEPOSITION FRACTIONS AND PARTICLE SURFACE AREA ANALYSIS}

Toxicity analysis, particle size distribution results and mass correlations have exhibited a strong relationship between metal fractions in the nanoparticle size bin to be influential in the PM number count emissions as well as toxicity responses. The analysis in the current section will provide results of deposition fractions for the different size bin together with total surface area and the lung deposited surface area, which is important in assessing health risks from heavy-duty natural gas engines. The results used deposition fraction equations developed by International Commission for Radiological Protection (ISRP). The dosimetry model representing human respiratory pathway and particle movements through it has been largely used by the health effect research to discern ambient particle transport to the lungs. The deposition fractions calculated for the different regions of the respiratory system namely 1 ) head and nasal airways $\left.\left(D_{\text {head }}\right) 2\right)$ tracheobronchial $\left.\left(D_{t b}\right) 3\right)$ aleveolar $\left(D_{\text {alveolar }}\right)$ will be used to calculate the lung deposited surface area that these regions will be subjected to. Since deposition frcation is a function of particle size, the results will illustrate deposition fraction for the three classified size bins of the particle size distribution.

Figure 5-37 shows the total deposition fraction and regional deposition fractions for the electrometer channels that represent the size ranges of the TSI EEPS. The ICRP deposition model used for this calculation represents averaged values for men and women for three different activity types that include resting, light exercise and heavy exercise. The illustration shows up to $90 \%$ total particle deposition in the respiratory system for particles in the size range of $5 \mathrm{~nm}$. However, this includes deposition in the head and nasal airways that are not as critical with respect to health effects implications as compared to particle deposition in trachea bronchial and alveolar. Hence, further results and LDSA calculation in this study will use only a sum of $D F_{\text {tb }}$ and $D F_{\text {alveolar }}$ fractions, due to their significance of particle deposition in the inner pathways and gas exchange regions of the lungs. 


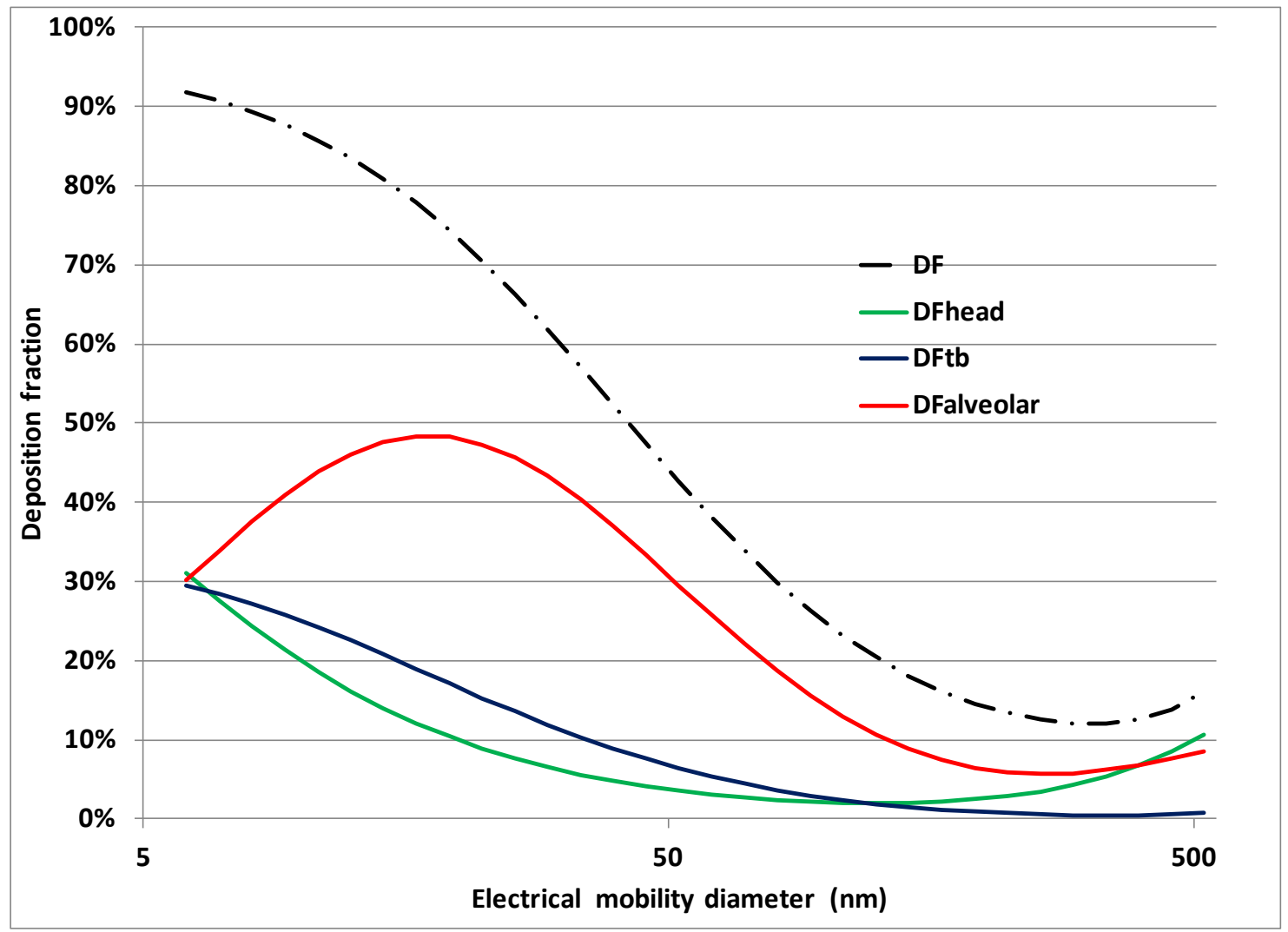

Figure 5-37 Regional and total deposition fractions calculated for the electrical mobility diameter range for the TSI EEPS instrument.

Table 24 lists the regional and total deposition fractions for the three different size bins of the particle size distribution measured over the UDDS cycle for bus 1 . The deposition fractions represented here are average over the entire driving cycle and represent the deposition of only the particle CMD as identified in section 5.4. 
Table 25 Regional deposition fractions for Bus 1 for the three different size bins over the UDDS cycle

\begin{tabular}{lc|cccc}
\hline \multicolumn{7}{c}{ Bus 1 UDDS } \\
\hline \multicolumn{7}{c}{ NP size Bin } \\
\hline Average & 6.11 & 0.917 & 0.309 & 0.293 & 0.304 \\
Std Dev & 0.501 & 0.006 & 0.014 & 0.005 & 0.016 \\
\hline \multicolumn{7}{c}{ UFP size Bin } \\
\hline \multicolumn{7}{c}{ DF } & DF $_{\text {head }}$ & DF $_{\text {tb }}$ & DF $_{\text {alveolar }}$ \\
\hline Average & 36.84 & 0.552 & 0.054 & 0.099 & 0.388 \\
Std Dev & 8.792 & 0.065 & 0.010 & 0.018 & 0.048 \\
\hline \multicolumn{7}{c}{ FP size Bin } \\
\hline \multicolumn{7}{c}{ Din } \\
\hline Average & 173.44 & 0.163 & 0.024 & 0.012 & 0.077 \\
Std Dev & 45.020 & 0.022 & 0.008 & 0.004 & 0.014 \\
\hline
\end{tabular}

Results from Bus 1 UDDS showed a total DF of 0.917 for an average CMD of $6.11 \mathrm{~nm}$ in the NP size bin and DF of 0.552 for an average CMD of $36.84 \mathrm{~nm}$. The FP size bin deposition can be considered insignificant because of the higher standard deviation in the measured CMD and low DF values. The NP size bin exhibits equal particle deposition fraction in both tracheobronchial and alveolar region of the lungs. The UFP size bin shows significant deposition in the alveolar region with a $\mathrm{DF}_{\text {alveolar }}$ of 0.388 in comparison to $\mathrm{DF}_{\mathrm{tb}}$ of 0.099 . Hence, suggesting the influence of elemental carbon if any on the alveolar tissues of the lungs.

Table 26 Regional deposition fractions for Bus 1 for the three different size bins over the steady state $45 \mathrm{MPH}$ cycle

\begin{tabular}{|c|c|c|c|c|c|}
\hline \multicolumn{6}{|c|}{ Bus 1 SS $45 \mathrm{MPH}$} \\
\hline \multicolumn{6}{|c|}{ NP size Bin } \\
\hline & CMD (nm) & DF & DF $_{\text {head }}$ & $D_{F_{t b}}$ & DF $_{\text {alveolar }}$ \\
\hline Average & 9.14 & 0.881 & 0.235 & 0.260 & 0.391 \\
\hline Std Dev & 2.267 & 0.030 & 0.061 & 0.025 & 0.065 \\
\hline \multicolumn{6}{|c|}{ UFP size Bin } \\
\hline & CMD (nm) & DF & $\mathbf{D F}_{\text {head }}$ & $D F_{t b}$ & DF $F_{\text {alveolar }}$ \\
\hline Average & 88.58 & 0.334 & 0.032 & 0.099 & 0.201 \\
\hline Std Dev & 32.690 & 0.162 & 0.018 & 0.018 & 0.125 \\
\hline \multicolumn{6}{|c|}{ FP size Bin } \\
\hline & CMD (nm) & DF & $\mathbf{D F}_{\text {head }}$ & $\mathrm{DF}_{\mathrm{tb}}$ & DF $_{\text {alveolar }}$ \\
\hline Average & 142.78 & 0.182 & 0.021 & 0.015 & 0.089 \\
\hline Std Dev & 3.100 & 0.004 & 0.000 & 0.001 & 0.003 \\
\hline
\end{tabular}


Table 25 lists the regional and total deposition fractions for the three different size bins of the particle size distribution measured over the steady state $45 \mathrm{MPH}$ cycle for bus 1 . The NP size bin reported a DF of 0.881 for an average CMD of $9.14 \mathrm{~nm}$ throughout the duration of the steady state operation. $\mathrm{DF}_{\mathrm{tb}}$ and $\mathrm{DF}_{\text {alveolar }}$ were calculated to be 0.26 and 0.391 respectively. The UFP size bin reported a DF of 0.334 with negligible deposition in the upper and tracheobronchial regions of the airways. The high variability in the measured CMD also indicates possible noise in measurement due to lower particle number count.

Table 27 Regional deposition fractions for Bus 1 for the three different size bins over the idle operation

\begin{tabular}{|c|c|c|c|c|c|}
\hline \multicolumn{6}{|c|}{ Bus 1 Idle } \\
\hline \multicolumn{6}{|c|}{ NP size Bin } \\
\hline & CMD (nm) & $\mathrm{DF}$ & $D_{F_{\text {head }}}$ & $\mathrm{DF}_{\mathrm{tb}}$ & $\mathrm{DF}_{\text {alveolar }}$ \\
\hline Average & 8.06 & 0.893 & 0.243 & 0.272 & 0.376 \\
\hline Std Dev & 0.000 & 0.000 & 0.000 & 0.000 & 0.000 \\
\hline \multicolumn{6}{|c|}{ UFP size Bin } \\
\hline & CMD (nm) & DF & $D_{F_{\text {head }}}$ & $D F_{t b}$ & $D_{\text {alveolar }}$ \\
\hline Average & 30.13 & 0.614 & 0.065 & 0.118 & 0.430 \\
\hline Std Dev & 5.803 & 0.034 & 0.005 & 0.009 & 0.026 \\
\hline \multicolumn{6}{|c|}{ FP size Bin } \\
\hline & CMD (nm) & DF & $D_{F_{\text {head }}}$ & $\mathrm{DF}_{\mathrm{tb}}$ & $\mathrm{DF}_{\text {alveolar }}$ \\
\hline Average & 127.16 & 0.203 & 0.021 & 0.019 & 0.106 \\
\hline Std Dev & 29.448 & 0.008 & 0.006 & 0.001 & 0.005 \\
\hline
\end{tabular}

Table 28 Regional deposition fractions for Bus 2 for the three different size bins over UDDS cycle

\begin{tabular}{|c|c|c|c|c|c|}
\hline \multicolumn{6}{|c|}{ Bus 2 UDDS } \\
\hline \multicolumn{6}{|c|}{ NP size Bin } \\
\hline & CMD (nm) & DF & DF $_{\text {head }}$ & $\mathrm{DF}_{\mathrm{tb}}$ & $D_{F_{\text {alveolar }}}$ \\
\hline Average & 7.26 & 0.903 & 0.271 & 0.281 & 0.343 \\
\hline Std Dev & 1.715 & 0.022 & 0.031 & 0.015 & 0.031 \\
\hline \multicolumn{6}{|c|}{ UFP size Bin } \\
\hline & CMD (nm) & DF & DF $_{\text {head }}$ & $\mathrm{DF}_{\mathrm{tb}}$ & DF $_{\text {alveolar }}$ \\
\hline Average & 67.75 & 0.396 & 0.037 & 0.061 & 0.263 \\
\hline Std Dev & 31.060 & 0.149 & 0.017 & 0.036 & 0.118 \\
\hline \multicolumn{6}{|c|}{ FP size Bin } \\
\hline & CMD (nm) & DF & $\mathrm{DF}_{\text {head }}$ & $\mathrm{DF}_{\mathrm{tb}}$ & $\mathrm{DF}_{\text {alveolar }}$ \\
\hline Average & 138.71 & 0.186 & 0.020 & 0.015 & 0.092 \\
\hline Std Dev & 8.185 & 0.009 & 0.000 & 0.001 & 0.007 \\
\hline
\end{tabular}


Table 29 Regional deposition fractions for Bus 2 for the three different size bins over the steady state $45 \mathrm{mph}$ cycle

\begin{tabular}{lccccc}
\hline \multicolumn{6}{c}{ Bus 2 SS 45 MPH } \\
\hline \multicolumn{7}{c}{ NP size Bin } \\
\hline Average & 6.55 & 0.911 & 0.297 & 0.288 & 0.316 \\
Std Dev & 1.483 & 0.019 & 0.039 & 0.016 & 0.042 \\
\hline \multicolumn{7}{c}{ UFP size Bin } \\
\hline \multicolumn{7}{c}{ CMD (nm) } & DF & DF $_{\text {head }}$ & DF $_{\text {tb }}$ & DF $_{\text {alveolar }}$ \\
\hline Average & 37.10 & 0.543 & 0.051 & 0.095 & 0.383 \\
Std Dev & 3.588 & 0.032 & 0.005 & 0.009 & 0.022 \\
\hline \multicolumn{7}{c}{ FP size Bin } \\
\hline \multicolumn{7}{c}{ DF } & DF $_{\text {head }}$ & DF $_{\text {tb }}$ & DF $_{\text {alveolar }}$ \\
\hline Average & 145.29 & 0.187 & 0.022 & 0.016 & 0.093 \\
Std Dev & 46.417 & 0.018 & 0.008 & 0.003 & 0.012 \\
\hline
\end{tabular}

Table 30 Regional deposition fractions for Bus $\mathbf{2}$ for the three different size bins over the idle operation

\begin{tabular}{|lccccc|}
\hline \multicolumn{7}{|c|}{ Bus 2 Idle } \\
\hline \multicolumn{7}{c|}{ NP size Bin } \\
\hline CMD (nm) & DF & DF $_{\text {head }}$ & DF $_{\text {tb }}$ & DF $_{\text {alveolar }}$ \\
\hline Average & 8.06 & 0.831 & 0.164 & 0.225 & 0.452 \\
Std Dev & 0.000 & 0.038 & 0.030 & 0.025 & 0.020 \\
\hline \multicolumn{7}{c|}{ UFP size Bin } \\
\hline Average & CMD (nm) & DF & DF $_{\text {head }}$ & DF $_{\text {tb }}$ & DF $_{\text {alveolar }}$ \\
Std Dev & 5.803 & 0.618 & 0.065 & 0.119 & 0.433 \\
\hline \multicolumn{7}{c}{0.000} & 0.000 & 0.000 & 0.000 \\
\hline & FP size Bin \\
\hline Average & 127.16 & 0.204 & 0.020 & 0.019 & 0.106 \\
Std Dev & 29.448 & 0.000 & 0.000 & 0.000 & 0.000 \\
\hline
\end{tabular}

Table 27 and Table 28 show the results of the regional deposition fraction calculation for the three different size bins for bus 2 over the UDDS cycle and the steady state $45 \mathrm{MPH}$ cycle respectively. The results show a similar deposition fraction values as observed from bus 1 due to the similarities in particle size distribution observed. However, a significant difference was 
observed during the idle operation of bus 2, where the particle size distribution resulted in a broader normal distribution curve encompassing both the NP and UFP size bin. As result the CMDs obtained for both size ranges where characterized by low standard deviation. The deposition fraction calculation indicates 0.831 and 0.618 total particle deposition for the NP and UFP size bin. Another interesting finding is that the alveolar deposition from both the NP and UFP size bin are very similar with 0.452 for NP size bin and 0.433 for UFP size bin. This is due to the fact that the CMD obtained for both size bins are within the particle diameter range that produce the highest deposition in the alveolar region. Since, the idle mode operation from bus 2 produced toxicity response that was an order of magnitude higher than other operating modes, the high alveolar deposition observed could result in high human lung tissue exposure to oxidative stress inducers.

Table 26 lists the regional and total deposition fractions for the three different size bins of the particle size distribution measured over the idle operation for bus 1 . NP size bin reported particle deposition fraction of 0.893 with $\mathrm{DF}_{\mathrm{tb}}$ and $\mathrm{DF}_{\text {alveolar }}$ calculated to be 0.272 and 0.376 respectively. The UFP size bin reported average particle CMD similar to that observed during the UDDS operation with an average CMD of $30.13 \mathrm{~nm}$ associated with a total deposition of 0.614. Alveolar deposition of particles with a $\mathrm{DF}_{\text {alveolar }}$ of 0.430 was the dominant deposition mechanism in this size bin.

Figure 5-38 shows the results of the total particle surface area calculation for the measured CMD in the different size bins for bus 1 operating over different drive cycles. The surface areas shown in Figure 5-38 will correspond to the particle surface are as emitted from the tailpipe of the vehicle. Figure 5-39 shows the results of the tracheobronchial and alveolar deposited surface area for the measured CMD in the different size bins for bus 1 over different drive cycles. The surface areas shown in Figure 5-39 will correspond to the particle surface are that will be exposed to the human tissue interactions. 


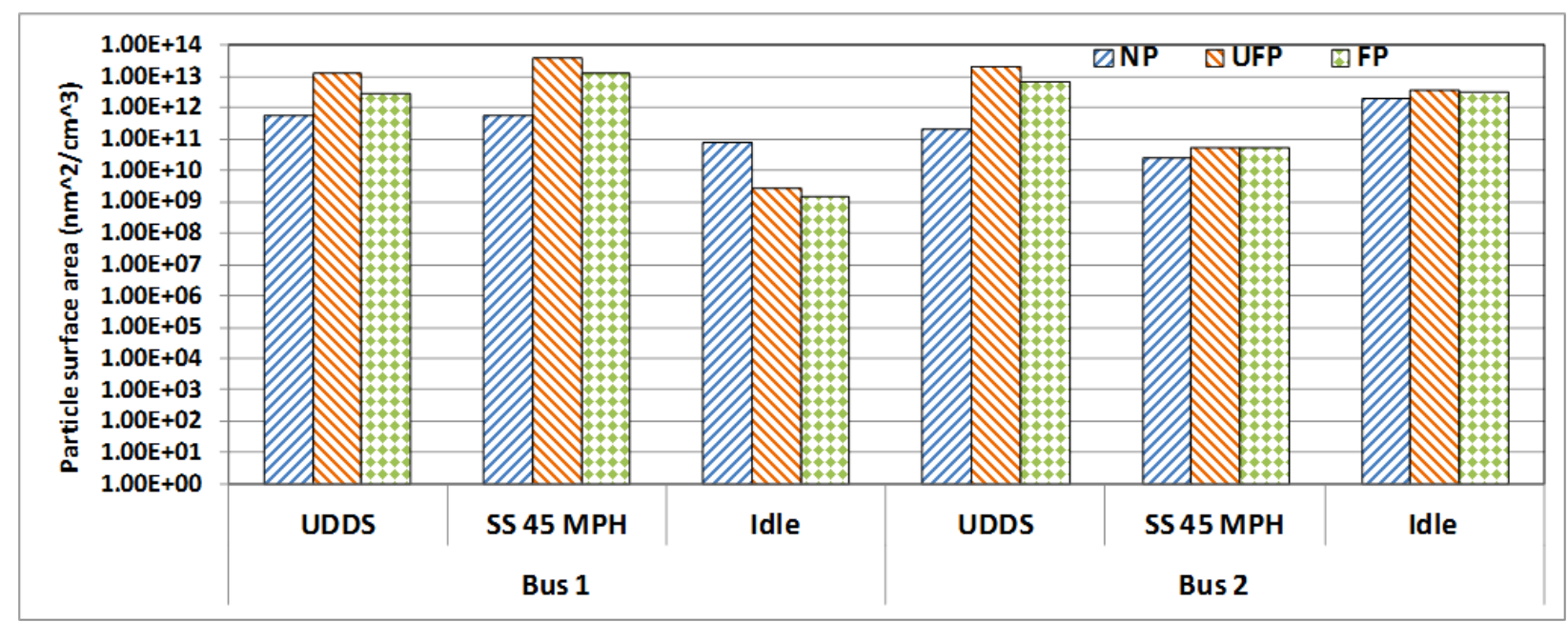

Figure 5-38 Total particle surface area calculated for the measured CMD in each size bin

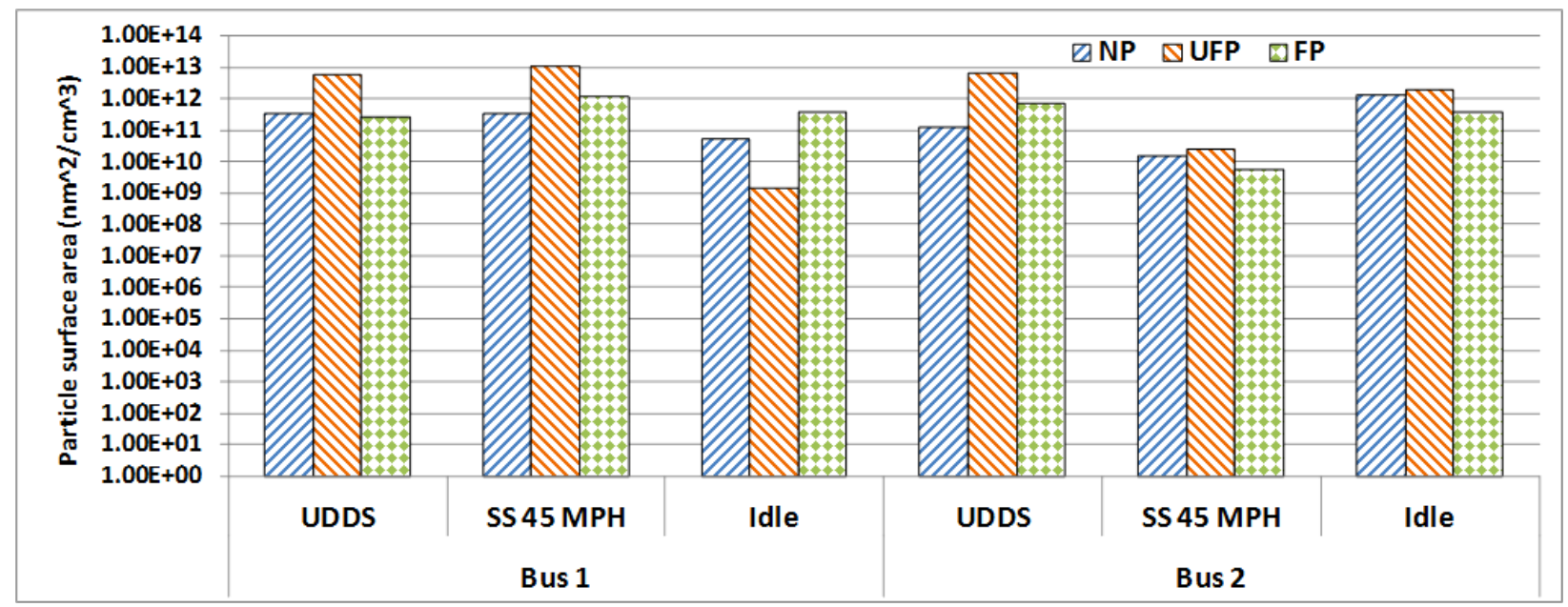

Figure 5-39 Tracheobronchial and alveolar deposited surface area calculated for the measured CMD in each size bin

As illustrated the results show maximum surface area of particulate matter for the CMD corresponding to the UFP size bin. The NP size bin surface area contribution is consistent through all operating modes. As observed in Figure 5-39 the lung deposited surface area (LDSA) for the NP and UFP size bins are of the same order of magnitude as observed in tailpipe owing to the high lung deposition in this size range. However, the LDSA of the FP size bin shows an order of magnitude change in particle surface area from that observed in the tailpipe.

Figure 5-40 show the particle surface area distribution representing engine out dilute particle size distribution and the surface area distribution calculated from applying the alveolar and tracheobronchial deposition fractions. The lung deposited particle surface area 
represented in the figure considers only the summation of the alveolar and tracheobronchial as deposition in the head and nasal airway can be considered as non critical with respect to health effects.

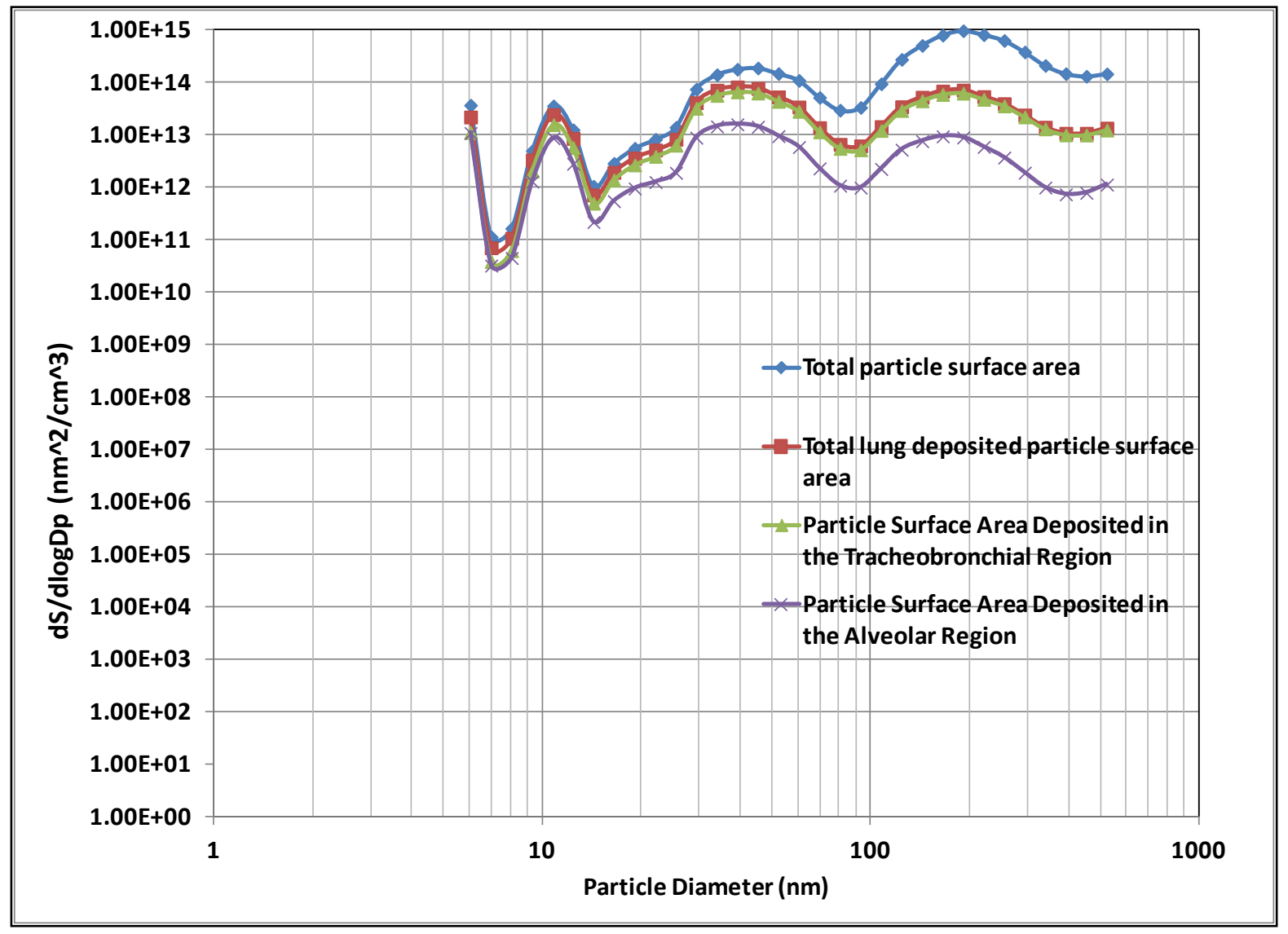

Figure 5-40 Particle surface area distribution, representing engine-out, lung deposited, tracheobronchial and alveolar deposition from bus 1 over the UDDS cycle.

The results shown for the UDDS cycle elucidates the significance of the nucleation mode distribution since almost the entire engine out particle surface area is exposed to the inner regions of the lung. The UFP and the FP mode particle suffer significant loss through impaction in the upper regions of the respiratory tract, hence decreasing its available surface area to the gas exchange regions by over two orders of magnitude. Particle size distribution analysis in the previous sections attributed particles in the FP size bin to possible dilution tunnel artifacts and not necessarily an engine out distribution. However, the UFP size range could be linked with elemental carbon fractions that were detected by $\mathrm{EC} / \mathrm{OC}$ analysis. 


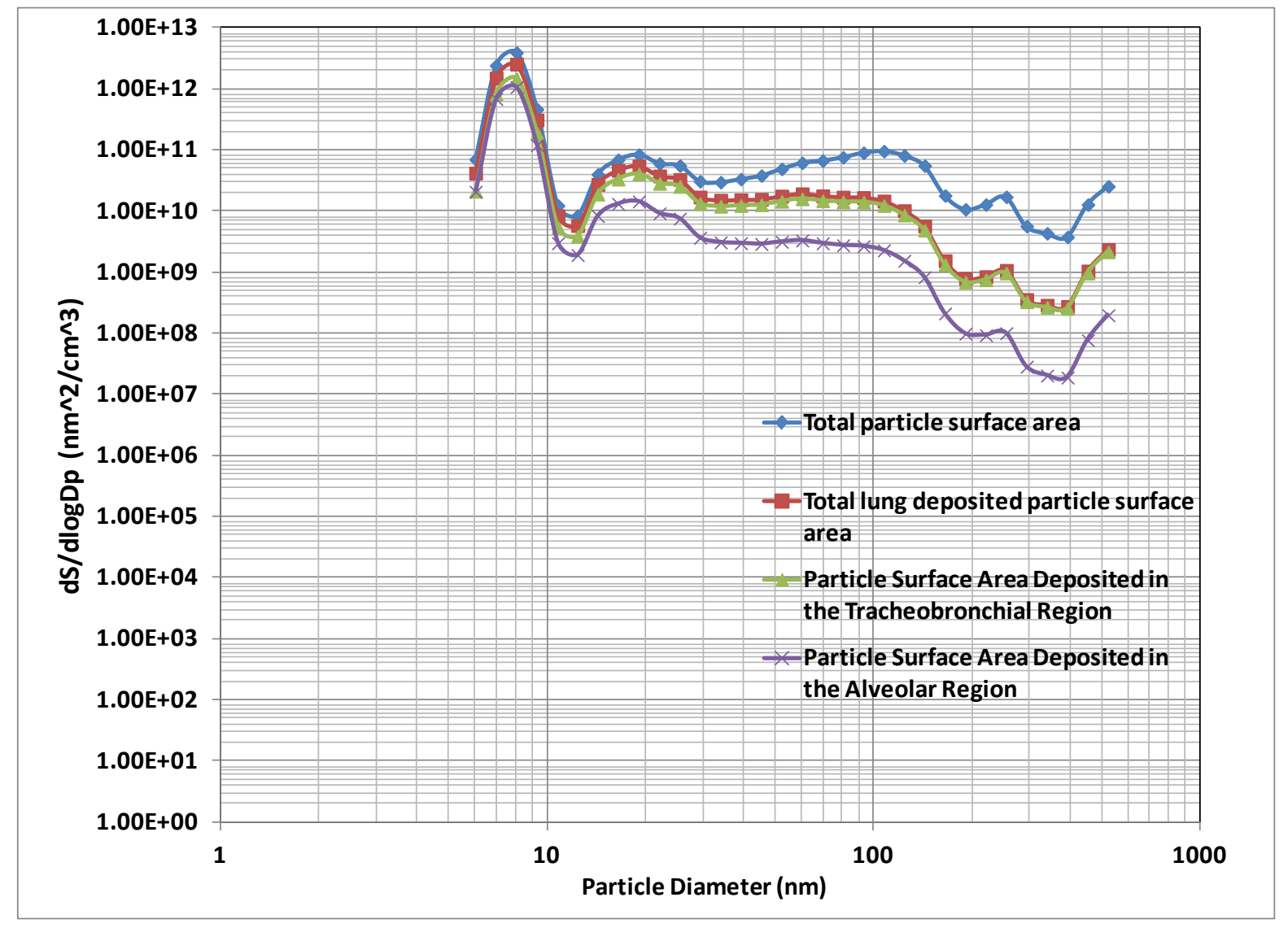

Figure 5-41 Particle surface area distribution, representing engine-out, lung deposited, tracheobronchial and alveolar deposition from bus 1 during Idle operation.

Figure 5-41 shows the particle surface area distributions for bus 1 during the idle operation. The results show a significant exposure to particle surface area in the NP size bin. Surface area deposition in the UFP and FP size bin are three orders of magnitude lower than the NP size bin.

Figure 5-42 shows the particle surface area distribution for bus 1 during the steady state 45 MPH operation. The particle size distribution and concentration measurement results obtained for this cycle resulted in the lowest particle number and mass emissions and as a result the number count measurements were characterized by higher levels of electrometer noise from the TSI EEPS. However, the nucleation mode surface area distribution was similar in magnitude to that observed during the UDDS cycle and idle operation. Since, the chemical speciation of gases and PM did not exhibit and volatile or inorganic fractions such as nitrates that could be contributing to higher particle size and greater mass, the surface area distribution in the UFP 
and FP size bin can be considered as not significant in relation to possible health effects due to particle toxicity.

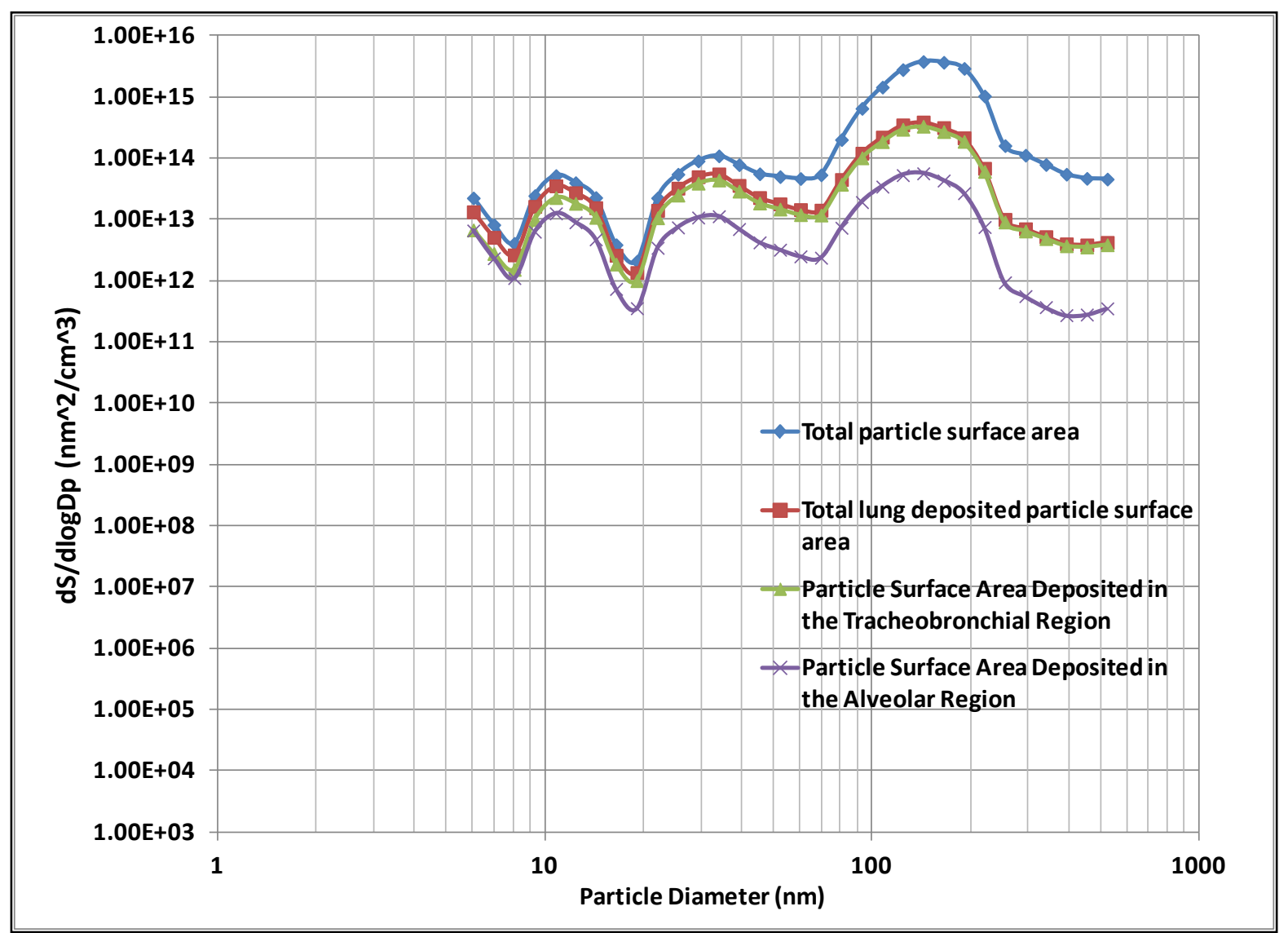

Figure 5-42 Particle surface area distribution, representing engine-out, lung deposited, tracheobronchial and alveolar deposition from bus 1 during steady state $\mathbf{4 5} \mathrm{mph}$ operation.

Figure 5-43 shows the particle surface area distribution from bus 2 over the UDDS cycle. Although the results show significant surface area contribution from UFP and FP size bins, chemical speciation of exhaust did not indicate any significant emissions of inorganic and carbon emissions that could be attributed to number count in that size range. Hence, the surface area distribution observed in the UFP and FP size category can be considered as possible sampling artifacts that may not reflect any adverse health effects of tailpipe nanoparticle emissions. Interestingly, the nanoparticle size bin, exhibited a surface area distribution with respect to magnitude and size range that was a similar to those observed from bus 1 during all driving cycles. 


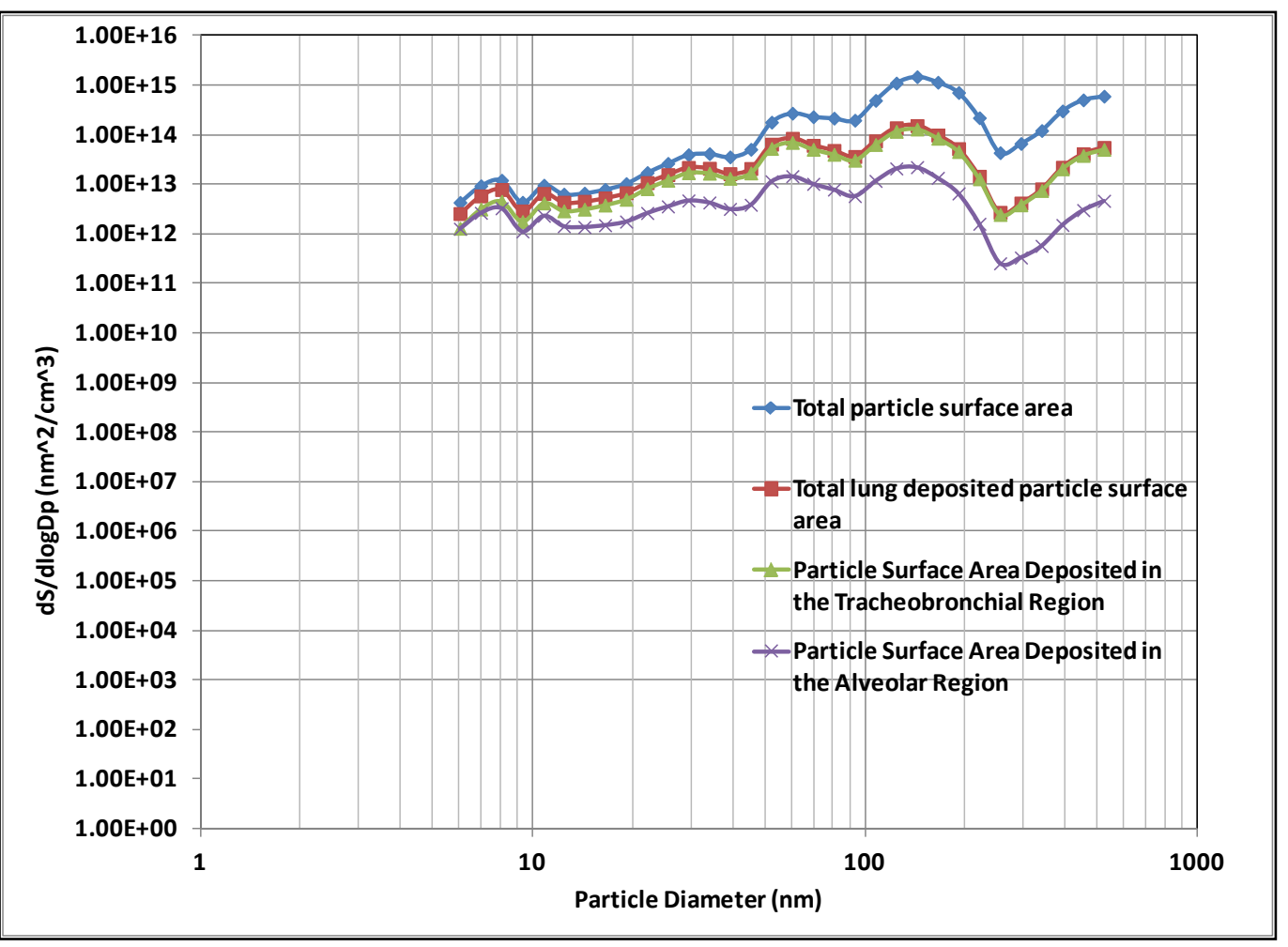

Figure 5-43 Particle surface area distribution, representing engine-out, lung deposited, tracheobronchial and alveolar deposition from bus 2 over the UDDS cycle.

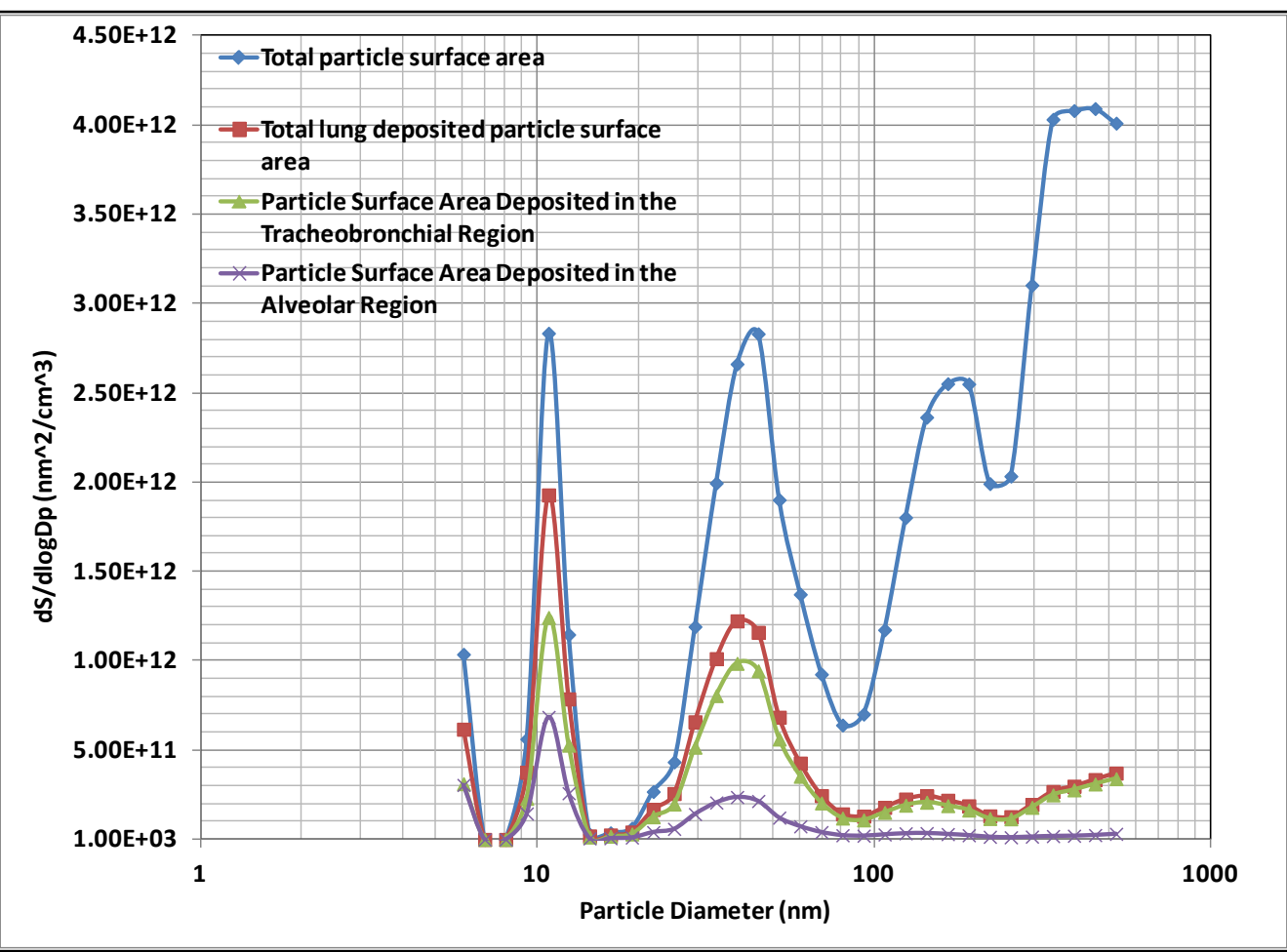

Figure 5-44 Particle surface area distribution, representing engine-out, lung deposited, tracheobronchial and alveolar deposition from bus 2 during steady state $45 \mathrm{mph}$ operation. 


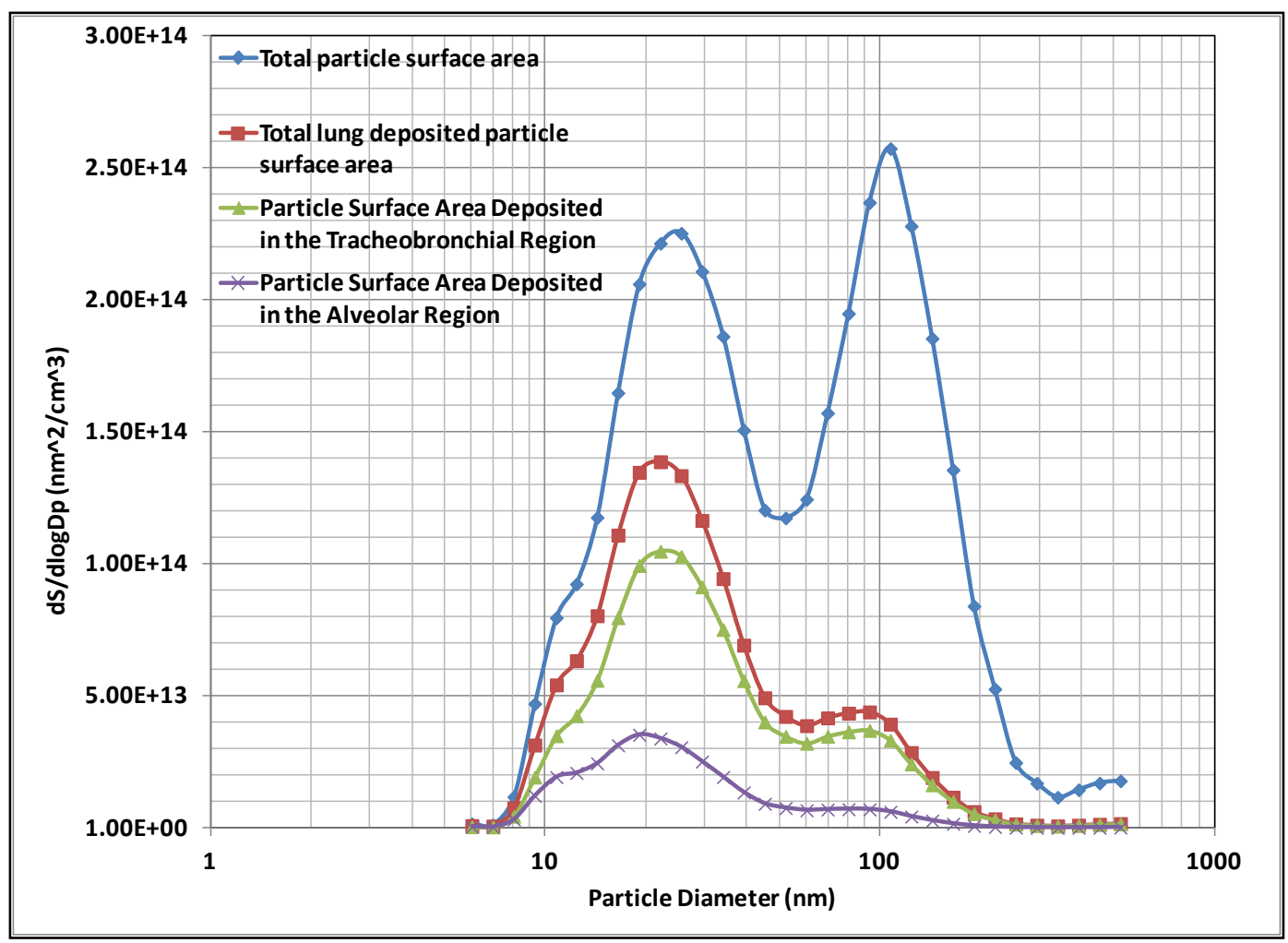

Figure 5-45 Particle surface area distribution, representing engine-out, lung deposited, tracheobronchial and alveolar deposition from bus 2 during idle operation.

Figure 5-44 shows the particle surface area distribution for the $45 \mathrm{MPH}$ steady state operation of bus 2. The total lung deposited surface area distribution shows an order of magnitude decrease in particle deposition for the FP size range. The NP size bin particle surface area deposited in the lungs is of the same order of magnitude as that observed from the tailpipe. This further elucidates the contribution of NP size bin particle to particle deposition in the lungs. A combination of higher toxicity potential and higher surface area for interaction could contribute to serious health effects.

Figure 5-45 shows the particle surface area distribution for the idle operation from bus 2 . The lung deposited surface area contribution for the idle operation is significant for particles within the UFP size range. Since, the idle operation resulted in higher emissions of EC, the UFP surface area illustrated here could be entirely contributed by EC fraction. It was observed in our toxicity analysis that EC fraction of the PM showed positive correlation towards the alveolar macrophage ROS assay. Although, we concluded that correlation needed a larger dataset to 
derive statistically significant conclusions, the surface area distribution deposited in the alveolar and tracheobronchial regions illustrate the importance of the UFP size range particles and its composition. A larger dataset will be required to quantify the EC composition of CNG exhaust and its associated toxicities to further illustrate the link the UFP size bin surface area deposition in the lungs to possible health effects.

\subsubsection{RELATIONSHIP BETWEEN ELECTRICAL AEROSOL DETECTOR RESPONSE TO PARTICLE SURFACE AREA}

The previous sections have illustrated the importance of particle surface area measurements and its regional deposition fractions, while relating toxicity responses to particle size distribution. However, measurement of lung deposited particle surface area can be achieved only through particle sizing equipments such as TSI EEPS for transient testing and TSI scanning mobility particle sizer (SMPS) for steady state testing. Measurement of particle surface area has traditionally been accomplished by primarily measuring the particle size distribution and concentration, and further calculating particle surface are by assuming spherical particle geometry. A recent study has shown an interesting relationship between diffusion charger based particle counter to particle surface area (Wilson et al., 2007). Diffusion charger based instruments are based on the principle of charge transfer to particles by mixing ionized air and particles. Charge transfer between particles and ionized air occur through the process of diffusion consequently number of particles can be measured through measurement of current from these ionized particles.

TSI electrical Aerosol Detector (TSI EAD) is a diffusion charger based instrument that reports a unique particle metric called as the aerosol length. Aerosol length can simply be viewed as the product of mean particle diameter (mono-modal distribution) and the total particle number count. However, the basic measurement principle of the EAD is the diffusion

based ionizing of particles and consequently measuring the electrometer current generated by impaction of these ionized particles. A recent work by Wilson et al. showed an interesting result that revealed high correlation of the raw EAD current to lung deposited surface area and regionally deposited particle surface area (Wilson et al., 2007). The findings of Wilson et al. is of high importance because, now the response of a diffusion charging based instrument can be 
directly correlated to lung deposited surface area. This also showed that the surface area offered by the particles is directly dependent on the charge acceptance by the particle through the process of diffusion. However, the study is based only on atmospheric particle size distribution and composition and further raises important questions regarding possible diffusion charging efficiencies for other particle size distribution and composition such as vehicle exhaust.

This section of the results will analyze the results of EAD measurements and particle surface area calculated from the various size bins of the particle size distribution to illustrate the correlation of EAD response to the lung deposited particle surface area.

Table 31 Pearson's correlation coefficient (R) and level of significance ( $p$ ) for correlation of EAD data Vs. regional and lung deposited surface area and particle number count in different size bins

\begin{tabular}{|c|c|c|}
\hline \multicolumn{3}{|l|}{ EAD Aerosol Length } \\
\hline & $\mathbf{R}$ & $\mathbf{p}$ \\
\hline NP size bin lung deposited surface area & 0.980 & 0.000 \\
\hline $\begin{array}{l}\text { NP size bin alveolar deposited surface } \\
\text { area }\end{array}$ & 0.980 & 0.000 \\
\hline $\begin{array}{l}\text { NP size bin Tracheobronchial deposited } \\
\text { surface area }\end{array}$ & 0.920 & 0.000 \\
\hline UFP size bin lung deposited surface area & 0.754 & 0.000 \\
\hline $\begin{array}{l}\text { UFP size bin alveolar deposited surface } \\
\text { area }\end{array}$ & 0.579 & 0.003 \\
\hline $\begin{array}{c}\text { UFP size bin Tracheobronchial deposited } \\
\text { surface area }\end{array}$ & 0.625 & 0.001 \\
\hline NP size bin total particle number & 0.690 & 0.000 \\
\hline UFP size bin total particle number & 0.481 & 0.010 \\
\hline
\end{tabular}

Table 30 shows the Pearson's correlation for average EAD response with lung, alveolar and tracheobronchial deposited surface area of each size bin of the particle size distribution for all vehicle operation. The results show high correlation coefficient with high statistical significance for EAD response and lung and regional deposition of particles of the NP size bin. However, the particle surface area in the UFP size bin showed only reasonable correlation for lung deposited and tracheobronchial and poor correlation with alveolar deposition. The poor correlation in the 
UFP region can be attributed to the lower particle concentration detected in this size range the possibility of sampling artifacts contributing to an inconsistent particle size distribution in the UFP and FP size bins.

EAD response with total particle number in the NP and UFP size bin correlated poorly, and further corroborating the existing theory of a diffusion chargers response being better correlated with particle surface are than particle number.

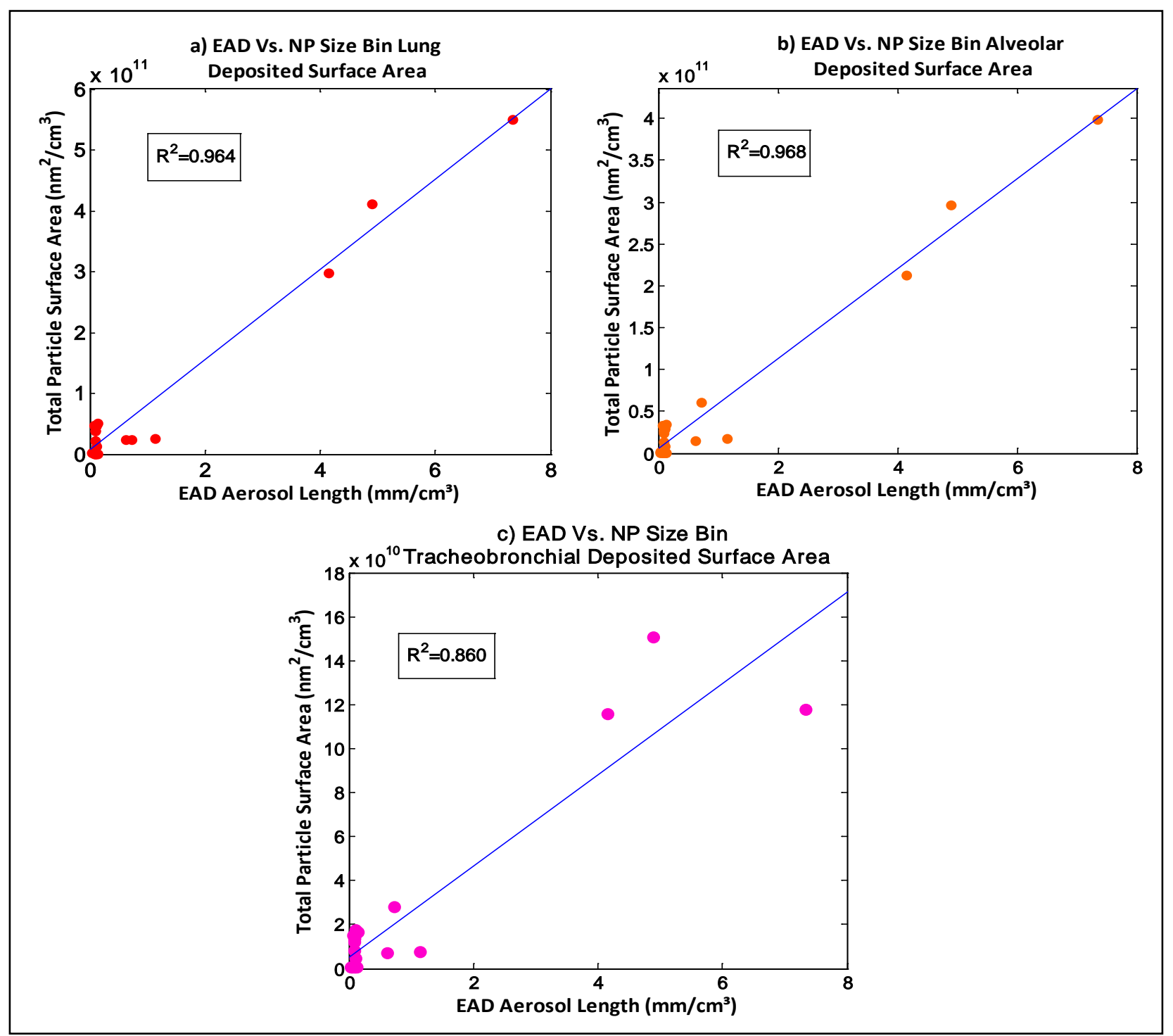

Figure 5-46 Scatter plot and linear fit of EAD data Vs. a) NP size bin total lung deposited particle surface area b) NP size bin alveolar deposited particle surface area c) NP size bin tracheobronchial deposited particle surface area 
Figure 5-46 shows the scatter plot with the liner fit for the EAD response with the total lung deposited surface area, alveolar deposited surface area and the tracheobronchial deposited surface area in the NP size bin. The illustration shows high correlation of EAD response to lung deposited surface area $\left(R^{2}=0.96\right)$, alveolar deposited surface area $\left(R^{2}=0.97\right)$ and tracheobronchial surface area $\left(R^{2}=0.86\right)$ in the NP size bin. The lung deposited surface area shown area considers only the summation of alveolar and tracheobronchial deposition fractions.

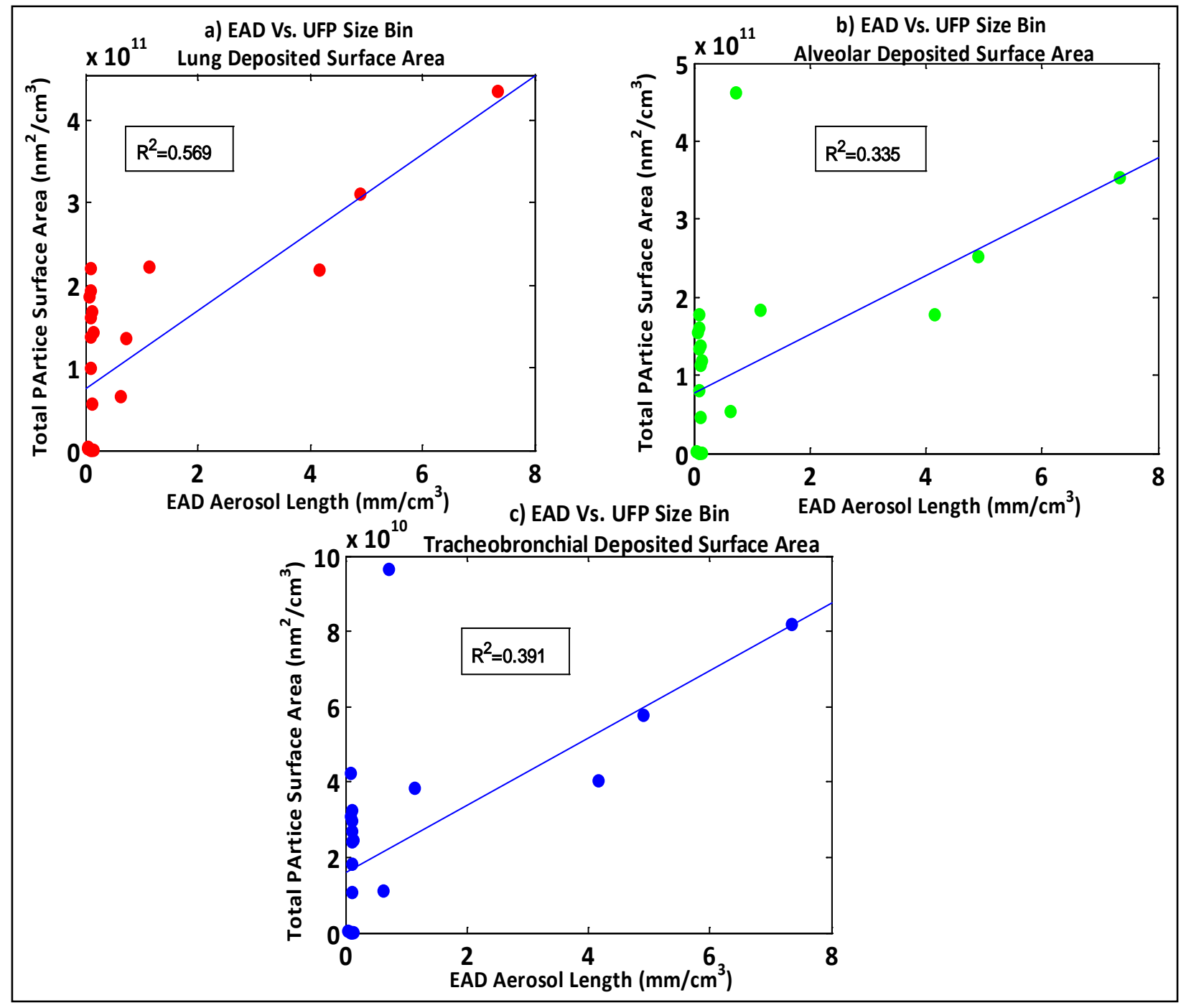

Figure 5-47 Scatter plot and linear fit of EAD data Vs. a) UFP size bin total lung deposited particle surface area b) UFP size bin alveolar deposited particle surface area c) UFP size bin tracheobronchial deposited particle surface area 
Figure 5-47 shows the scatter plot with the liner fit for the EAD response with the total lung deposited surface area, alveolar deposited surface area and the tracheobronchial deposited surface area in the UFP size bin. The illustration shows the poor correlation exhibited by the surface area deposition in the UFP size bin with the EAD response.

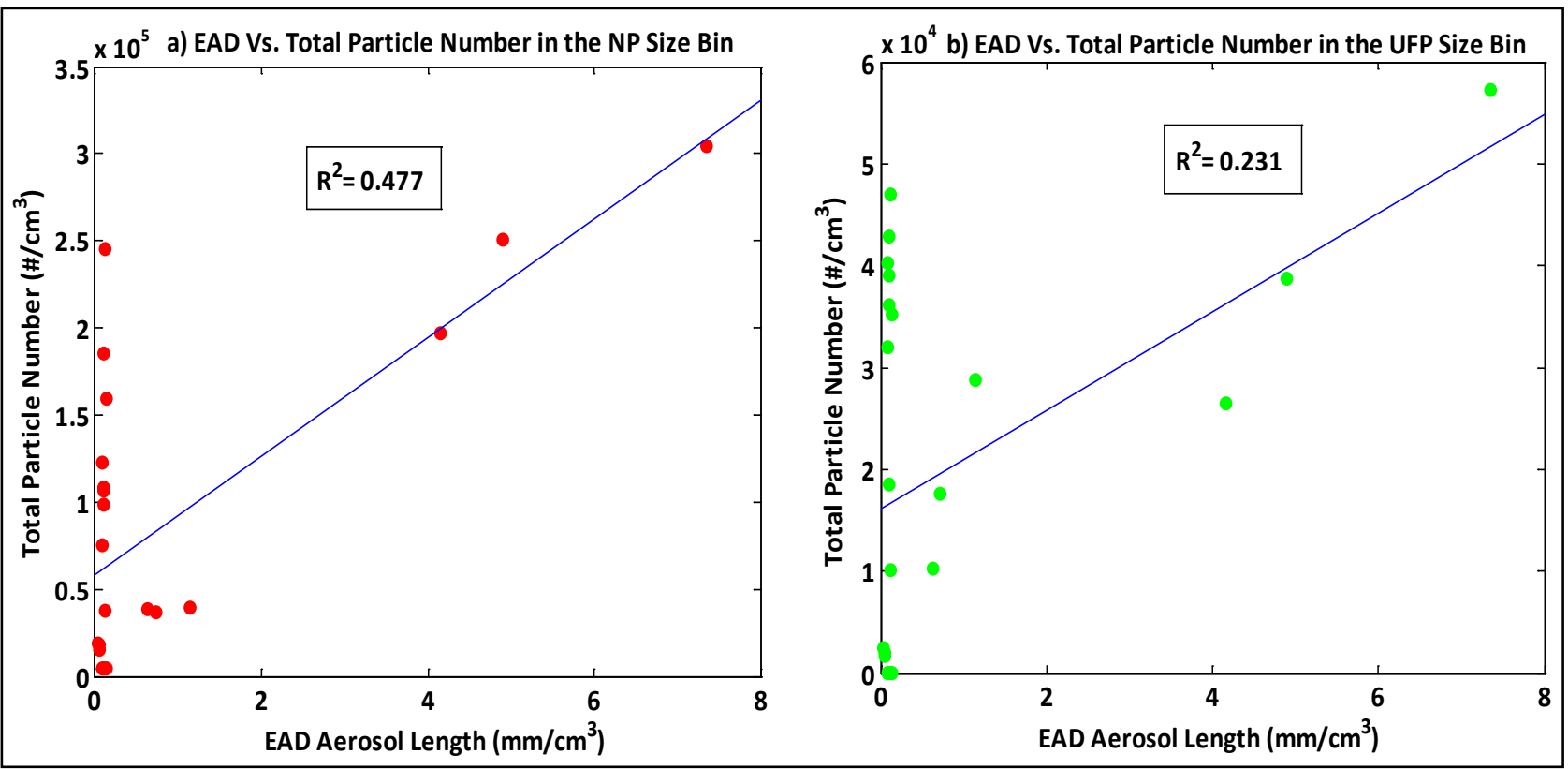

Figure 5-48 Scatter plot and linear fit of EAD data Vs. a) Total particle number count in the NP size bin b) Total particle number count in the UFP size bin

Figure 5-48 shows the correlation scatter plot between EAD response and total particle number count in the NP and UFP size bin. The FP size bin has been omitted for this correlation due to the uncertainties in particle size measurement due to low particle concentration and possibility of results being biased by sampling artifacts.

The results of this analysis show the ability to use the response of an EAD or any diffusion charging instrument to predict the lung deposited surface area of a particle stream. Hence, toxicity responses can be directly correlated with a simple diffusion charging instrument's response rather than the use of complex size and number count measurement instrumentation. 


\section{CHAPTER 6 - CONCLUSIONS}

The key objectives of this research to chemically characterize of the gas phase and particulate phase of the tailpipe emissions from heavy-duty natural gas engines was successfully carried out. The results further established the toxicity profile of thermally denuded and non-denude particulate matter and correlated observed toxicity with elemental and nanoparticle emissions.

The gas phase chemical speciation results reported all carbonyl, PAH and VOC emissions close to levels found in background or below the detection limits of the analytical method. The results of the gas phase speciation indicated no significant contribution to particle formation through homogenous nucleation or through vapor phase condensation on solid particulate matter. The findings of this study further challenge the existing view of high volatile and semivolatile fractions in the tailpipe of advanced heavy-duty natural gas engines. The results indicate that high exhaust temperature conditions, characteristic of a stoichiometric engine results in almost consistent catalytic activity of all hydrocarbons, and semi-volatile compounds with exception to methane. This sustained high efficiency catalytic activity results in lower particulate forming volatile compounds such $\mathrm{PAH}$ and carbonyl compounds which are considered to precursors to exhaust particle formation during dilution of vehicle exhaust.

Physical characterization of the PM yielded important information of the EC/OC split of the total PM emissions. OC emissions and VOC chemical speciation results by indicated a volatile organic fraction both in particulate phase and gas phase to be close to detection limits over most operating conditions. The OC concentrations reported for certain transient conditions could be a result of sampling artifacts due to the affinity of sampling media (pre-fired quartz) to absorption of gas phase hydrocarbons mainly in the form of methane. Interestingly the study reported significant EC emissions rate during idle operation that could be directly attributed to combustion of lubrication oil in the combustion chamber.

Elemental and ion analyses were also performed on the PM samples to further discern the non volatile fraction PM emitted from these vehicles. The results indicated a significant mass emission rates of metal and elements that can be linked to additives used in lubrication oil. 
Elements and metals such as $\mathrm{Ca}, \mathrm{P}, \mathrm{K}, \mathrm{Zn}, \mathrm{S}$ and $\mathrm{Mg}$ were found in significant concentration in the PM samples. The findings of the study directly relate lubrication oil as the single most dominant source to non-volatile fraction PM emissions in the tailpipe.

The primary objective of the study was to discern the toxicity potential of the volatile and non-volatile fraction of PM from advanced heavy-duty natural gas engines. The low mass emission rate of PM from these engines proved to be a major challenge in collecting PM for toxicity analysis. Toxicologists typically require close to a milligram of PM depending on the assay to obtain a valid toxicity response that can be considered statistically significant. Hence, the study utilized high-volume sampling to collect larger PM mass in shorter test duration. However, it is to be noted that increase in sampling volume will always be associated with sampling artifacts such as increased gas adsorption onto the filter material that could bias the mass specific toxicity results. Hence, the study has refrained from using a mass specific toxicity metric and has focused more on relating toxicity to various fractions of PM. Three different toxicity assays were used in this study namely a) DHBA b) DTT and c) ROS macrophage assay. The DHBA and DTT assay are chemical based assays that are highly sensitive to metal and elemental component of PM. The ROS assay does not have any specific affinity to a certain fraction and can be considered to be a representative of PM toxicity as a whole. Results of the PM indicated toxicity values lower than those reported earlier in literature of legacy and DPFSCR equipped diesel vehicles. This partly due to the fact that the PM emissions observed from advanced natural gas engines are characterized by low mass emission rates. However, certain operating conditions resulted in toxicity responses that were enough to draw statistically significant conclusions. Both DHBA and DTT assay correlated highly with mass of elements and metals such as $\mathrm{Zn}, \mathrm{Fe}$ and Co. The DTT assay resulted in high correlation with mass of $\mathrm{Cu}, \mathrm{Zn}, \mathrm{P}$ and PAH with molecular weight less than 200. The ROS assay also resulted in high statistical correlation with mass of $\mathrm{Zn}, \mathrm{P}$ and $\mathrm{Cr}$. The ROS assay statistically well correlated with EC fraction. However, lack of sufficient EC mass from natural gas engines imparts high level of uncertainty in the observed statistical correlation with ROS toxicity. Literature also lacks sufficient information on the toxicity response of ROS assay to EC fraction. Hence a larger dataset with larger non-zero EC mass result with corresponding ROS responses could further 
shed light on this observed statistical correlation. The results showed that the toxicity responses on a whole were statistically well correlated with mass of metals emissions from the natural gas vehicles. High exhaust temperatures resulted in VOC emissions close to detection limits resulting in no statistical correlation between VOC concentrations and toxicity responses, further indicating no contribution of VOCs to particle formation in stoichiometrically fueled natural gas engines. However, PAH emissions of compounds lower than 200 in molecular weight showed statistically good correlation with the DHBA assay indicating the possibility of PAH adsorbing or condensing on solid fractions of PM such as metals, elements and EC fractions.

The vehicles were tested over three driving cycles namely triple length UDDS, steady state $45 \mathrm{mph}$ and idle mode operation. Continuous particle size and concentration measurements were performed using the TSI EEPS. Particle size distribution measurements were presented to discern the particle formation mechanisms upon dilution of the exhaust of advanced natural gas engines. The results of the particle size distribution showed an consistent nucleation mode peak, independent of the vehicle operation and exhaust temperature conditions to be present. No significant accumulation mode particle size distribution was observed during UDDS or steady-state vehicle operation from both vehicles. However, idle operation from bus 2 showed significant mass loading on the filter with significant particle concentrations in the accumulation region of the particle size distribution. The study also divided the entire particle size distribution measured by the TSI EEPS into three size ranges namely NP size bin (6.04 nm-23.3 nm), UFP size bin (25.4 nm -100.1 nm) and FP size bin (112.3 nm to $500 \mathrm{~nm})$.

Once the study established statistically significant correlation between mass of metals and elemental with all three toxicity assays, the study further presented an analysis of the bin wise particle size derived mass to the mass of elemental and metal fractions in the exhaust. The statistically significant correlation of mass of metals to the NP size bin presented in this study, suggests the possibility of nucleation mode size particles to primarily consist of lubrication oil additives. Oil analysis report the presence of the similar elemental composition in the oil samples as that observed in the PM samples further corroborating the conclusion of lubrication 
oil as the primary source driving metallic emissions and consequently the toxicity of PM in heavy-duty natural gas engines.

The findings of the study the report the possibility of non-volatile nanoparticles in the size range of $10 \mathrm{~nm}$ is important from a regulatory perspective of enforcing number count regulation. Natural gas engine technology and after treatment configuration is significantly different from that of current model diesel, due to the absence of an exhaust particulate filter. Since, natural gas combustion is soot free, a particulate filtered is not required to meet PM regulations. Nucleation mode particles in diesel technology are usually related to nucleation of sulfates and water to form sulfuric acid particles. However a stoichiometric natural gas engine is devoid of excess oxygen in the exhaust and consequently prevents the $\mathrm{SO}_{3}$ formation. However, statistical correlations presented in this study indicate the possibility of nucleation mode particles of non-volatile metallic and elemental particles. Similar, findings were observed in a study by Lee et al. (Lee et al., 2006). The source of the precursors for this type of nucleation can be directed towards the lubrication oil consumption in these soot free engines transported via piston rings or more predominantly through the crank case ventilation system inducted in to the intake charge air of the engines. This type of homogenous nucleation may not be representative of only natural gas engines, and may be prevalent in diesel technology too. However, such particle formation would not be observed in a DPF equipped diesel due to the filtration process of the particulate filter, and the high soot content upstream of the DPF that would result mask this particle formation mechanism.

The possibility of existence of sub $23 \mathrm{~nm}$ non-volatile particles challenges existing protocol such as the European PMP that regulates solid particle number count above the $23 \mathrm{~nm}$ size range. The repeatability of this particle size distribution in the nucleation region indicates a strong and consistent contribution of lubrication oil to non-volatile PM fraction in the nucleation region, especially for vehicles equipped with crank case ventilation inducted in to the intake air. The study concludes that tailpipe toxicity of PM is strongly driven by metallic and elemental compositions that are believed to be in the nucleation region of the particle size distribution. Although all toxicity assay tested in this study were more of a bulk analysis of total PM mass extracted, the observed particle size distribution in the nucleation region suggests the 
possibility of greater penetration of these non-volatile particles in to the human respiratory system. The high lung deposited surface area offered by these particles could contribute to higher levels of oxidative stress in lung and respiratory tissues thereby increasing health risks.

Atmospheric studies have previously illustrated the strong correlation of diffusion charger response to particle surface area (Wilson et al., 2007). However, no study had previously illustrated a similar correlation for engine exhaust. The findings of this study clearly show the correlation between particle surface area and diffusion charger response clearly exists for engine exhausts also that is different from atmospheric PM in physical and chemical characteristics. The diffusion charger response in this study being that of an electrical aerosol detector shows statistically significant correlation with the total lung deposited surface area. With recent development in diffusion charger sensors such as the Pegasor Particle Sensor (PPS), tailpipe monitoring of particle charge is much more direct with no elaborate sampling systems. Hence, the findings of this study further concludes that a lung deposited surface area based metric for exhaust particle monitoring could be directly achieved through a tailpipe diffusion charger and can more appropriately relate to health effects in terms surface area exposure and dosage. 


\section{REFERENCES}

1998. Diesel and Gasoline Engine Exhausts and Some Nitroarenes. International Agency for Research On Cancer.

2012. IARC: Diesel Engine Exhaust Carcinogenic. 12 June 2012.

ANDERSON, J., PRESTON, H., WARRENS, C. \& BRETT, P. 2004. Fuel and Lubricant Effects on Nucleation Mode PArticle Emissions from a Euro III Light Duty Diesel Vehicle. SAE Paper2004-01-1989.

ANDERSSON, J., MAMAKOS, A., GIECHASKIEL, B., CARRIERO, M. \& MARTINI, G. 2010. Particle Measurement Programme (PMP) Heavy-Duty Inter-laboratory Correlation Exercise-Final Report. Ispra, Italy: European Commision- Joint Research Centre; Institute for Environment and Sustainability.

ARDANESE, R. 2008. Control of NOx and PM emissions from SCR-equipped 2010 compliant Heavy-Duty Diesel Engine over Different Engine-Out Calibrations. Doctor of Philosophy, West Virginia University.

ARDANESE, R., ARDANESE, M., BESCH, C. M., ADAMS, R. T., THIRUVENGADAM, A., SHADE, B. \& GAUTAM, M. 2009. PM Concentrations and Size Distributions from a Heavy-duty Diesel ENgine Programmed with Different Engine-out Calibrations to meet the 2010 Emission Limits. SAE, 2009-01-1183.

ASA-AWUKU, A. A. 2008. Characterizing Water-Soluble Organic Aerosol And Their Effects On Cloud Droplet Formation: Interactions of Carbonaceous Matter With Water Vapor. Doctor of Philosophy, Gerogia Institute of Technology.

AyAlA, A., GeBEL, M. E., OKAMOTO, R. A., REIGER, P. L., KADO, N. Y., COTTER, C. \& VERMA, N. 2003. Oxidation catalyst effect on CNG transit bus emissions. SAE, 2003-01-1900.

AYALA, A., KADO, N. Y., OKAMOTO, R. A., HOLMEN, B. A., KUZMICKY, P. A., KOBAYASHI, R. \& STIGLITZ, K. E. 2002. Diesel and CNG heavy-duty transit bus emissions over multiple driving schedules: Regulated pollutants and project overview. SAE, 2002-01-1722.

BAGELY, S. T., BAUMGAURD, K. J., GRATZ, L. D., JOHNSON, J. H. \& LEDDY, D. G. 1996. Characterization of Fuel and After-treatment Device Effects on Diesel Emissions. Health Effects Institute Research.

BAO, L., SEKIGUCHI, K., WANG, Q. \& SAKAMOTO, K. 2009. Comparison of Water-Solube Organic Components in Size-Segregated Particles between a Roadside and a Suburban Site in Saitama, Japan. Aerosol and Air Quality Research, 9, 412-420.

BISWAS, S., VERMA, V., SCHAUER, J. J., CASSEE, R. F., CHO, K. A. \& SIOUTAS, C. 2009a. Oxidative Potential of semi-Volatile and Non-Volatile Particulate Matter (PM) from Heavy-Duty Vehicles Retrofitted with Emission Control Technologies. Environmental Science and Technology, 43, 3905-3912. 
BISWAS, S., VERMA, V., SCHAUER, J. J. \& SIOUTAS, C. 2009b. Chemical Speciation of PM Emissions from Heavy-Duty Diesel Vehicles Equipped with Diesel Particulate Filter (DPF) and Selective Catalytic Reduction (SCR) Retrofits. Atmospheric Environment, 43, 19171925.

BURTSCHER, H. 2001. Tailpipe Particulate Emission Measurement for Diesel Engines. Windisch, Switzerland: Fachhochschule Aargau, University of Applied Science.

CHARRIER, J. G. \& ANASTASIO, C. 2012. On Dithiothreitol (DTT) as a Measure of Oxidative Potential for Ambient Particles: Evidence for the Importance of Soluble Transition Metals. Atmospheric Chemistry and Physics, 12, 11317-11350.

CHO, K. A. \& FROINES, R. J. 2006. The Chemical Toxicology of Particulate Matter. Los Angeles: University of California.

CHO, K. A., SIOUTAS, C., MIGUEL, H. A., KUMAGAI, Y., SCHMITZ, A. D., SINGH, M., EIGURENFERNANDEZ, A. \& FROINES, R. J. 2005. Redox activity of airborne particulate matter at different sites in the Los Angeles Basin. Environmental Research, 99, 40-47.

COUDRAY, C. \& FAVIER, A. 2000. Determination of salicylate hydroxylation products as an in vivo oxidative stress marker. Free Radical Biology and Medicine, 29, 1064-1070.

DEFOORT, M., OLSEN, D. \& WILSON, B. 2003. The effect of air-fuel ratio control strategies on nitrogen compound formation in three-way catalysts. International Journal of Engine Research, 5, 115-122.

DISTEFANO, E., EIGUREN, F. A., DELFINO, J. R., SIOUTAS, C., FROINES, R. J. \& CHO, K. A. 2009. Determination of metal-based hydroxy radical generating capacity of ambient and diesel exhaust particles. Inhalation Toxicology, 21, 731-738.

DOCKERY, D. W., POPE III, C. A., XU, X., SPENGLER, J. D., WARE, J. H., FAY, M. E., FERRIS JR., B. G. \& SPEIZER, F. E. 1993. An association between air pollution and mortality in six U. S. cities. New England Journal of MEdicine, 329, 1753-1759.

ENGLERT, N. 2004. Fine Particles and Human Health: A Review of Epidemiological studies. Toxicology Letters, 149, 235-242.

ENSOR, S. D. 2011. Aerosol Science and Technology: Histroy and Reviews. Research Triangle Institute.

FROELUND, K., OWENS, W. C., FRAME, E., BUCKINGHAM, J. P., GARBAK, J., TSEREGOUNIS, S. \& JACKSON, A. 2001. Impact of Lubrication Oil on Regulated Emissions of a Light Duty Mercedes Benz )M611 CDI Engine. SAE paper-2001-01-1901.

FUJITA, M. E., CAMPBELL, E. D. \& ZIELINSKA, B. 2006. Chemical Analysis of Lubrication Oil Samples from a Study to Characterize Exhaust EMissions from Light-Duty Gasoline Vehicles in the Kansas City Metropolitan Area. Reno, NV: Desert Research Institute.

FUJITA, M. E., ZIELINSKA, B., CAMPBELL, E. D., ARNOTT, P. E., SAGEBIEL, J., MAZZOLENI, L., CHOW, J., GABELE, P., CREWS, W., SNOW, R., CLARK, N., WAYNE, S. W. \& LAWSON, D. 2007. Varitaions in Speciated Emissions from Spark-Iginition and Compression-Ignition 
Motor Vehicles in Calfornia's South Coast Air Basin. Journal of the Air and Waste Management Association, 57, 705-720.

GEORGE, S. 2008. Investigation of Lubricant oil consumption and its contribution to particulate matter emissions. Doctor of Philosophy, West Virginia University.

GIECHASKIEL, B., BUENO, M. R., RUBINO, L., MANFREDI, U., DILARA, P. \& SANTI, D. G. 2007. Particle Measurement Programme (PMP): Particle Size and Number Emissions Before, During and After Regeneration Events of a Euro 4 DPF Equipped Light-Duty Diesel Vehicle. SAE, SAE 2007-01-1944.

GIECHASKIEL, B., DILARA, P., SANDBACH, E. \& ANDERSSON, J. 2008. Particle Measurement Programme (PMP) Light-Duty Inter-Laboratory Exercise: Comparison of Different Particle Number Measurement Systems. Measurement Science and Technology, 19.

HEI 2010. A Critical Review of the Literature on EMissions, Exposure and Health Effects. Boston: Health Effects Institute.

HERNER, D. J., HU, S., ROBERTSON, H. W., HUAI, T., CHANG, O. M. C., REIGER, P. L. \& AYALA, A. 2011. Effect of Advanced Aftertreatment for PM and NOx Reduction on Heavy-Duty Diesel Engine Ultrafine PArticle Emissions. Environmental Science and Technology, 45, 2413-2419.

HOSONUMA, K., YOSHIDA, K. \& MATSUNAGA, A. 1985. The Decomposition Products of Zinc Dialkyldithiophosphate in an Engine and Their Interaction with Diesel Soot. Wear, 103, 297-309.

HU, S., POlIDORI, A., ARHAMI, M., SHAFER, M. M., SCHAUER, J. J., CHO, K. A. \& SIOUTAS, C. 2008. Redox Activity and Chemical Speciation of Size Fractioned PM in the Communities of the Los Angeles-Long Beach Harbor. Atmospheric Chemistry and Physics, 8, 64396451.

JANSEN, L. K., LARSON, V. T., KOENIG, Q. J., MAR, F. T., FIELDS, C., STEWART, J. \& LIPPMANN, M. 2005. Associations between Health Effects and Particulate Matter and Black Carbon in Subjects with Respiratory Disease. Environmental Health Perspectives, 113, 1741-1746.

JOHNSON, K. C., DURBIN, T. D., JUNG, H., CHAUDHARY, A., DAVID, C. R., HERNER, D. J., ROBERTSON, W. H., HUAI, T., AYALA, A. \& KITTELSON, D. B. 2009. Evaluation of the European PMP Methodologies during On-Road and Chassis Dynamometer Testing for DPF Equipped Heavy-Duty Diesel Vehicles. Aerosol Science and Technology, 43, 962-969.

KHALEK, A. I. 2007. 2007 Diesel Particulate Measurement Research- E-66 Phase 3 Final Report. San Antonio, TX: Southwest Research Institute.

KHALEK, A. I., KITTELSON, D. B., GRASKOW, B. R. \& WEI, Q. 1998. Diesel Exhaust Particle Size: Measurement Issues and Trends. SAE, 980525.

KITTELSON, D. B. 1998a. Engine and Nanoparticles: A Review. Journal of Aerosol Science, 29, 575-588. 
KITTELSON, D. B. 1998b. Engines and Nanoparticles: A Review. Journal of Aerosol Science, 29, 575-588.

KITTELSON, D. B., WATTS, W. \& JOHNSON, J. 2002. Diesel Aerosol Sampling Methodology -CRC E-43. Coordinating Research Council.

KITTELSON, D. B., WATTS, W. F., JOHNSON, J. P., THORNE, C., HIGHAM, C., PAYNE, M., GOODIER, S., WARRENS, C., PRESTON, H., ZINK, U., PICKLES, D., GOERSAMNN, C., TWIGG, M. V., WALKER, A. P. \& BODDY, R. 2008. Effect of fuel and lube oil sulfur on the performance of a diesel exhaust gas regenerating trap. Environmental Science and Technology, 42, 9276-9282.

LANDREMAN, P. A., SHAFER, M. M., HEMMING, C. J., HANNIGAN, P. M. \& SCHAUER, J. J. 2012. A Macrophage-Based Method for the Assessment of the REactive Oxygen Species (ROS) Activity of Atmospheric Particulate Matter (PM) and Application to Routine (Daily-24h) Aerosol Monitoring Studies. Aerosol Scence and Technology, 42, 946-957.

LEE, D., MILLER, A., KITTELSON, D. B. \& ZACHARIAH, M. R. 2006. Charcaterization of metalbearing diesel nanoparticles using single-particle mass spectrometry. Journal of Aerosol Science, 37, 88-110.

LEV-ON, M., LETAVEC, C., UIHLEIN, J., KIMURA, K., ALLEMAN, T. L., LAWSON, D. R., VERTIN, K., GAUTAM, M., THOMPSON, G. J., WAYNE, S. W., CLARK, N., OKAMOTO, R. A., RIEGER, P., YEE, G., ZIELINSKA, B., SAGEBIEL, J., CHATTERJEE, S. \& HALLSTROM, K. 2002. Speciation of organic compounds from the exhaust of trucks and buses: Effect of fuel and aftertreatment on vehicle emissions profile. $S A E, 2002-01-2873$.

LI, N., SIOUTAS, C., CHO, K. A., SCHMITZ, A. D., MISRA, C., SEMPF, J., WANG, M., ODERLEY, T., FROINES, R. J. \& NEL, A. 2003. Ultrafine Particulate Pollutants Induce Oxidative Stress and Mitochondrial Damage. Environmental Health Perspectives, 111, 455-460.

LI, R., NING, Z., CUI, J., KHALSA, B., AI, L., TAKABE, W., BEEBE, T., MAJUMDAR, R., SIOUTAS, C. \& HSIAI, T. 2009. Ultrafine Particles from Dlesel Engines Induce Vascular Oxidative Stress Via JNK Activation. Free Radical Biology and Medicine, 46, 775-782.

LI, R., NING, Z., MAJUMDAR, R., CUI, J., TAKABE, W., JEN, N., SIOUTAS, C. \& HSAI, T. 2010. Ultrafine particles from diesel vehicle emissions at different driving cycles induce differential vascular pro-infalmmatory responses: Implication of chemical componenets and NF-kB signaling. Particle and Fibre Toxicology, 7.

LIU, G. Z., VASYS, N. V., DETTMANN, E. M., SCHAUER, J. J., KITTELSON, D. B. \& SWANSON, J. 2012. Comparison of Strategies for the MEasurement of MAss Emissions from Diesel Engines EMitting Ultra-Low Levels of Particulate Matter. Aerosol Scence and Technology, $43,1142-1152$.

LIU, Y.-Q., KEANE, M., ENSELL, M., MILLER, W., KASHON, W., ONG, T., MAUDERLY, J., LAWSON, D., GAUTAM, M., ZIELINSKA, B., WHITNEY, K. \& WALLACE, W. 2005. In-Vitro Genotoxicity of Exhaust Emissions of Diesel and Gasoline Engine Vehicles Operated on a Unified Driving Cycle. Journal of Environmental Monitoring, 7, 60-66. 
MAJEWSKI, A. 2005. Selective Catalytic Reduction [Online]. http://www.dieselnet.com/tech/cat scr.php. [Accessed 5/10/2012 2012].

MARICQ, M. \& XU, N. 2004. The Effective Density and Fractal Dimension of Soot PArticles from PRemixed Flames and Motor Vehicle Exhaust. Aerosol Science, 35, 1251-1274.

MCMILLIAN, M. H., CUI, M., GAUTAM, M., KEANE, M., ONG, T., WALLACE, W. \& ROBEY, E. 2002. Mutagenic Potential of Particulate Matter from Diesel Engine Operation on FischerTropsch as a Function of Engine Operating Conditions and Particulate Size. SAE, SAE 2002-01-1699.

NAKASHIMA, K., ISHIHARA, S. \& URANO, K. 1995. Influence of Piston Ring Gaps on Lubricant Oil FLow into the Combustion Chamber. SAE paper-952546.

NEMMAR, A., HOET, P. H. M., VANQUICKENBORNE, B., DINSDALE, D., THOMEER, M., HOYLAERTS, M. F., VANBILLOEN, H., MORTELMANS, L. \& NEMERY, B. 2002. Passage of Inhaled Particles into the Blood Circulation in Humans. Circulation, 411-414.

NTZIACHRISTOS, L., FROINES, R. J., CHO, K. A. \& SIOUTAS, C. 2007. Relationship between redox activity and chemical speciation of size-fractioned particulate matter. Particle and Fibre Toxicology, 4.

OBERDORSTER, G., FERIN, J. \& LEHNERT, B. E. 1994. Correlation Between Particle Size, In Vivo Particle Persistence, and Lung Injury. Environmental Health Perspectives, 102, 173-179.

POPE III, C. A., THUN, M. J., NAMBOODIRI, M. M., DOCKERY, D. W., EVANS, J. S., SPEIZER, F. E. \& HEATH JR., C. W. 1995. Particulate air pollution as a predictor of mortality in a prospective study of U.S. adults. American Journal of Respiratory and Critical Care Medicine, 151, 669-674.

RISTOVSKI, D. Z., MILJEVIC, B., SURAWSKI, C. N., MORAWSKA, L., FONG, M. K., GOH, F. \& YANG, A. I. 2012. Respiratory Health Effects of Diesel Particulate MAtter. Respirology, 17, 201212.

SAGER, M. T. \& CASTRANOVA, V. 2009. Surface Area of Particles Administered Versus Mass in Determining the Pulmonary Toxicity of Ultrafine and Fine Carbon Black: Comparison to Ultrafine Titanium Dioxide. Particle and Fibre Toxicology, 6.

SAKURAI, U., TOBIAS, H. J., PARK, K., ZARLING, D., DOCHERTY, S., KITTELSON, D. B., MCMURRY, P. H. \& ZIEMANN, P. J. 2003. On-Line Measurements of Diesel Nanoparticle Composition and Volatility. Atmospheric Environment, 379, 1199-1210.

SEAGRAVE, J., GIGLIOTTI, A., MCDONALD, D. J., SEILKOP, S., WHITNEY, K., ZIELINSKA, B. \& MAUDERLY, J. 2005. Composition, Toxicity, and Mutagenicity of Particulate and Semivolatile Emissions. Toxicological Sciences, 871, 232-241.

SHI, X. C., KEANE, M., ONG, T., HARRISON, J. C., SLAVEN, J. E., BUGARSKI, A. D., GAUTAM, M. \& WALLACE, W. 2005. Diesel Exhaust PArticulate Matter Dispersed in a Phospholipid Surfactant Induces Chromosomal Aberrations And Micronuclei But Not 6-ThioguanineResistant Gene Mutation In V79 Cells. Journal of Toxicology and Environmental Health, Part A, 68, 431-444. 
SUBHASIS, B., VISHAL, V., JAMES, J. S. \& CONSTANTINOS, S. 2009. Chemical Speciation of PM emissions from heavy-duty diesel vehicles equipped with diesel particulate filter (DPF) and Selective Catalytic Reduction (SCR) retrofits. Atmospheric Environment, 43, 19171925.

THIRUVENGADAM, A., BESCH, C. M., CARDER, D., OSHINUGA, A. \& GAUTAM, M. 2012. Influence of Real-World Engine Load Conditions on Nanoparticle Emissions from a DPF and SCR Equipped Heavy-Duty Diesel Engine. Environmental Science and Technology, 46, 19071913.

THIRUVENGADAM, A., CARDER, D., KRISHNAMURTHY, M. \& GAUTAM, M. Year. Comparison of Regulated and Unregulated Exhaust Emissions from a Fleet of Multi-Fuel Solid Resource Collection Vehicles. In: ASME Internal Combustion Engine Division, 2010 Fall Technical Conference, 2010 San Antonio, TX.

USEPA 2012. Code of Federal Regulations, Title 40, Part 1065 Engine-Testing Procedures. 57437-57470

VAARASLAHTI, K., KESKINEN, J., GIECHASKIEL, B., SOLLA, A., MURTONEN, T. \& VESALA, H. 2005. Effect of Lubricant on the Formation of Heavy-Duty Diesel Exhaust Nanoparticles. Environmental Science and Technology, 39, 8497-8504.

VERMA, V. 2011. Toxicological Characteristics of Particulate Matter in an Urban Environment and their Linakge to the Source Specific Constituents. Doctor of Philosophy, University of Southern California.

VERMA, V., NING, Z., CHO, K. A., SCHAUER, J. J., SHAFER, M. M. \& SIOUTAS, C. 2009. Redox Activity of Urban Quasi-Ultrafine particles from Primary and Secondary Sources. Atmospheric Environment, 43, 6360-6368.

VERMA, V., SHAFER, M. M., SCHAUER, J. J. \& SIOUTAS, C. 2010. Contribution of transition metals in the reactive oxygen species activity of PM emissions from retrofitted heavyduty vehicles. Atmospheric Environment, 44, 5165-5173.

WICHMANN, H. E. \& PETERS, A. 2000. Epidemilogical evidence of the effects of ultrafine particle exposure. Philosophical transactions of the Royal Society, 358, 2751-2769.

WILSON, E. W., STANEK, J., HAN, H.-S., JOHNSON, T., SAKURAI, H., PUI, Y. H. D., TURNER, J., CHEN, D.-R. \& DUTHIE, S. 2007. Use of the Electrical Aerosol Detector as an Indicator of the Surface Area of Fine Particles Deposited in the Lung. Journal of Air and Waste Management Association, 57, 211-220.

WU, C.-Y. \& ALLEN, A. Respiratory Deposition [Online]. University of Florida. Available: http://aerosol.ees.ufl.edu/respiratory/section05.html [Accessed 6/29/2012 2012].

ZIELINSKA, B., SAGEBIEL, J., MCDONALD, D. J., WHITNEY, K. \& LAWSON, D. R. 2004. Emission Rates and Comparitive Chemical Composition from Selected In-USe Diesel and GasolineFueled Vehicles. Journal of the Air and Waste Management Association, 54, 1138-1150. 
166 | P a g e 


\section{APPENDIX I- TSI EEPS PARTICLE SIZE DISTRIBUTION}

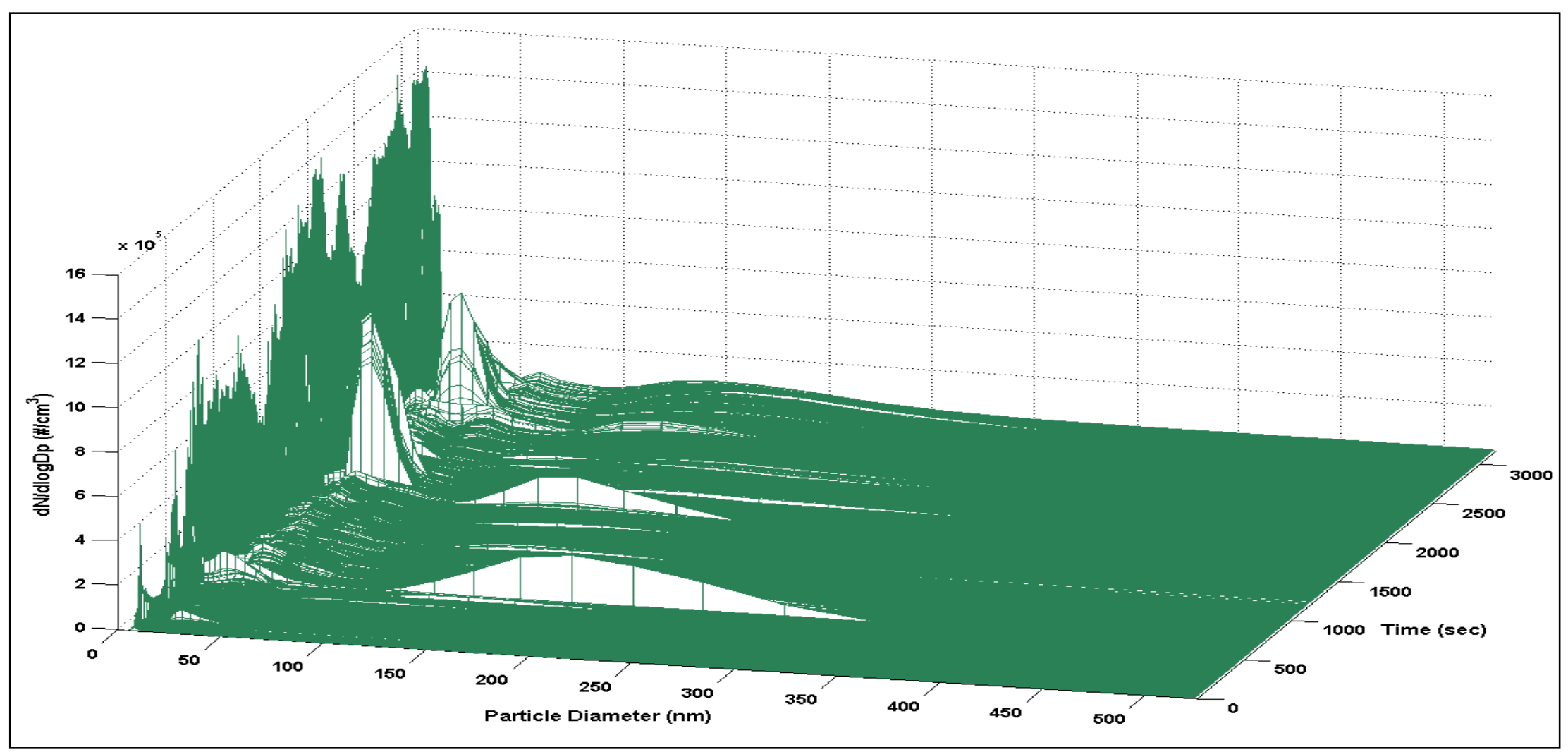

Bus 1 UDDS instantaneous particle size distribution measured with TSI EEPS (not corrected for dilution ratio)

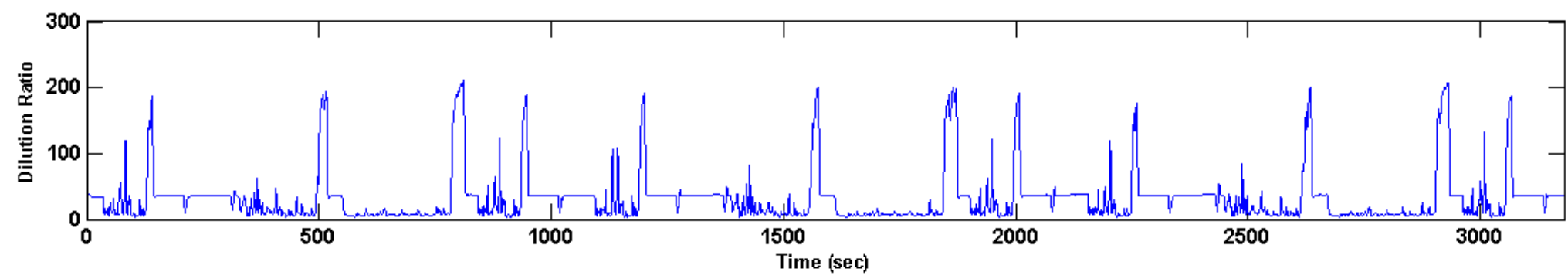

Bus 1 UDDS average instantaneous CVS dilution ratios 


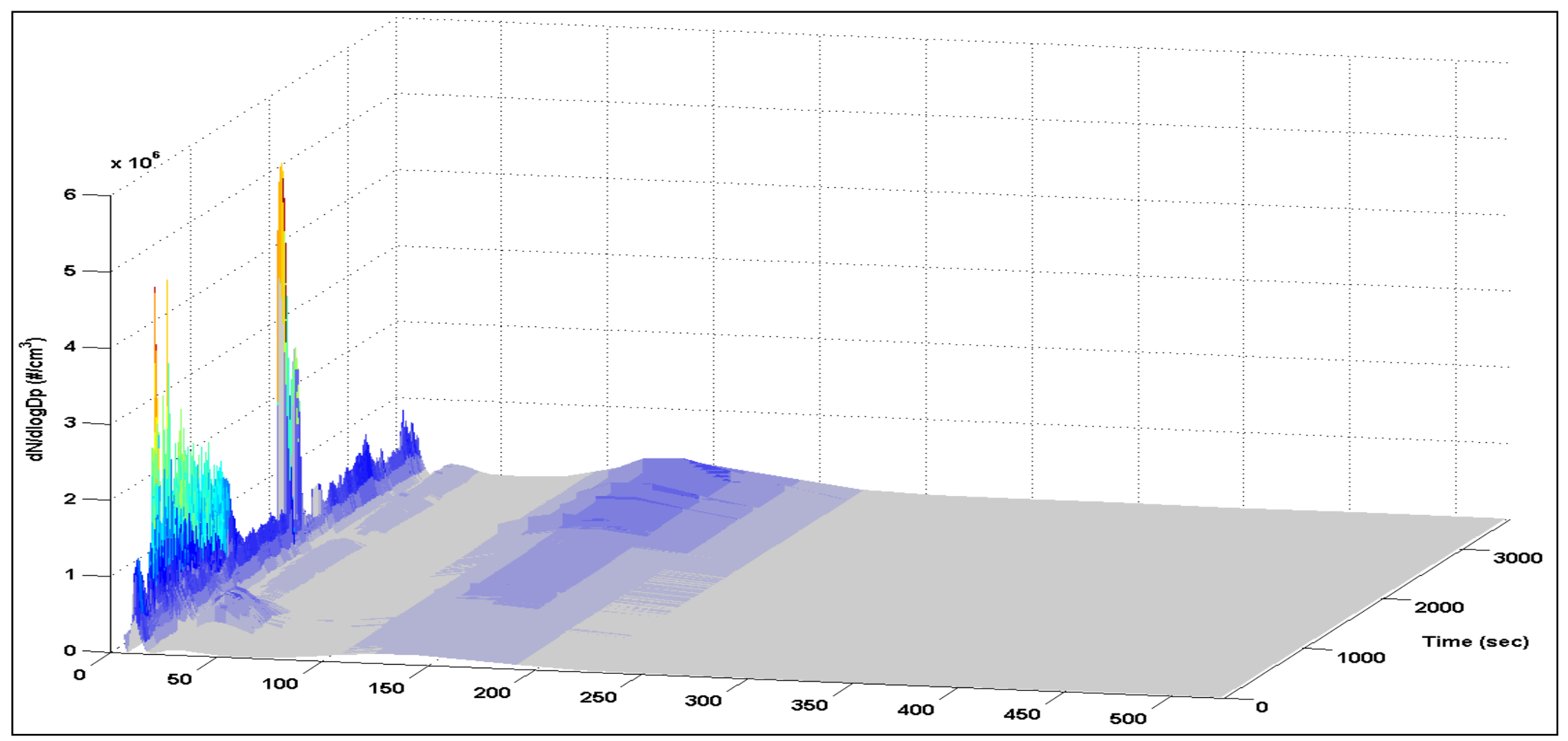

Bus 1 SS 45MPH instantaneous particle size distribution measured with TSI EEPS (not corrected for dilution ratio)

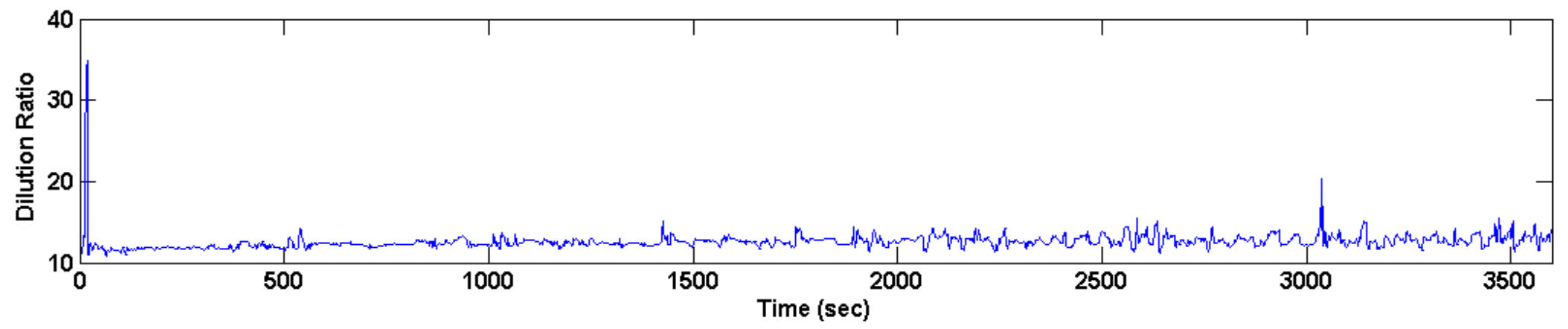

Bus 1 SS 45MPH average instantaneous CVS dilution ratios 


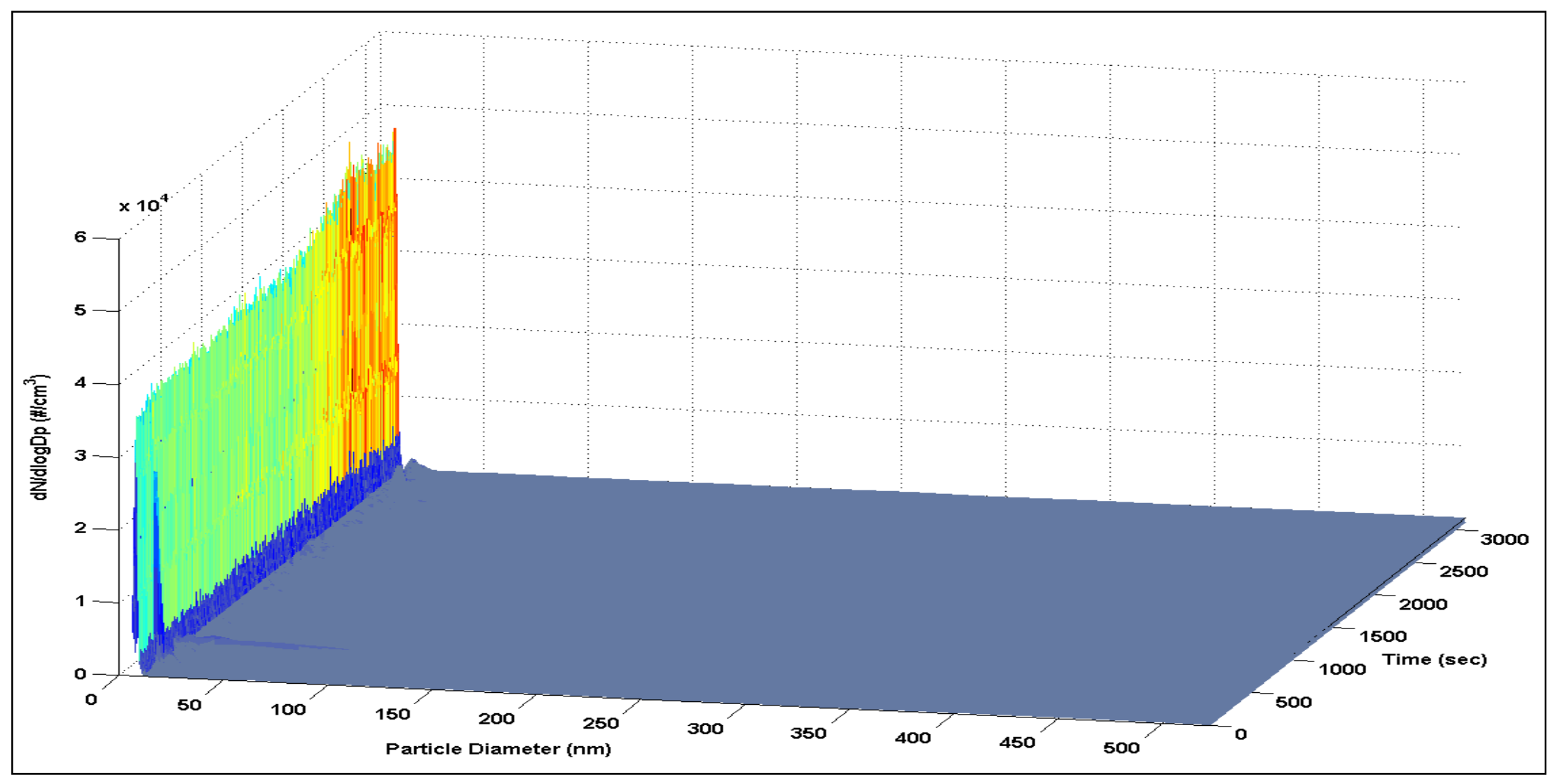

Bus 1 idle instantaneous particle size distribution measured with TSI EEPS (not corrected for dilution ratio)

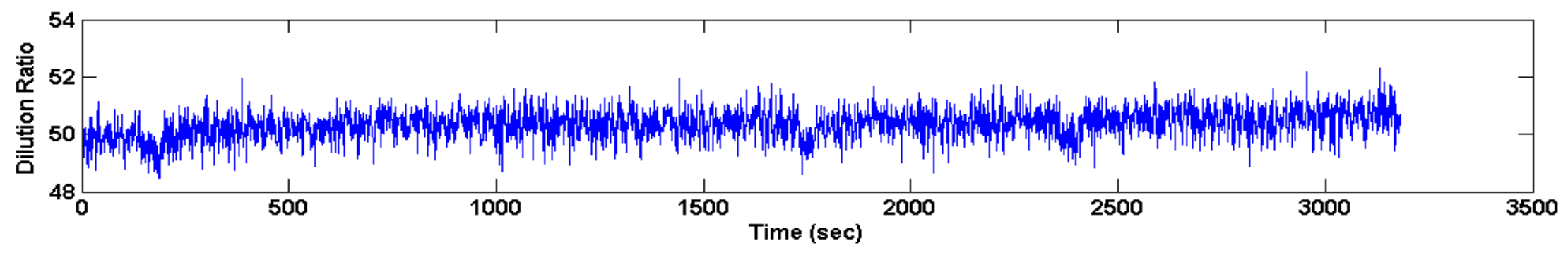

Bus 1 idle average instantaneous CVS dilution ratios 


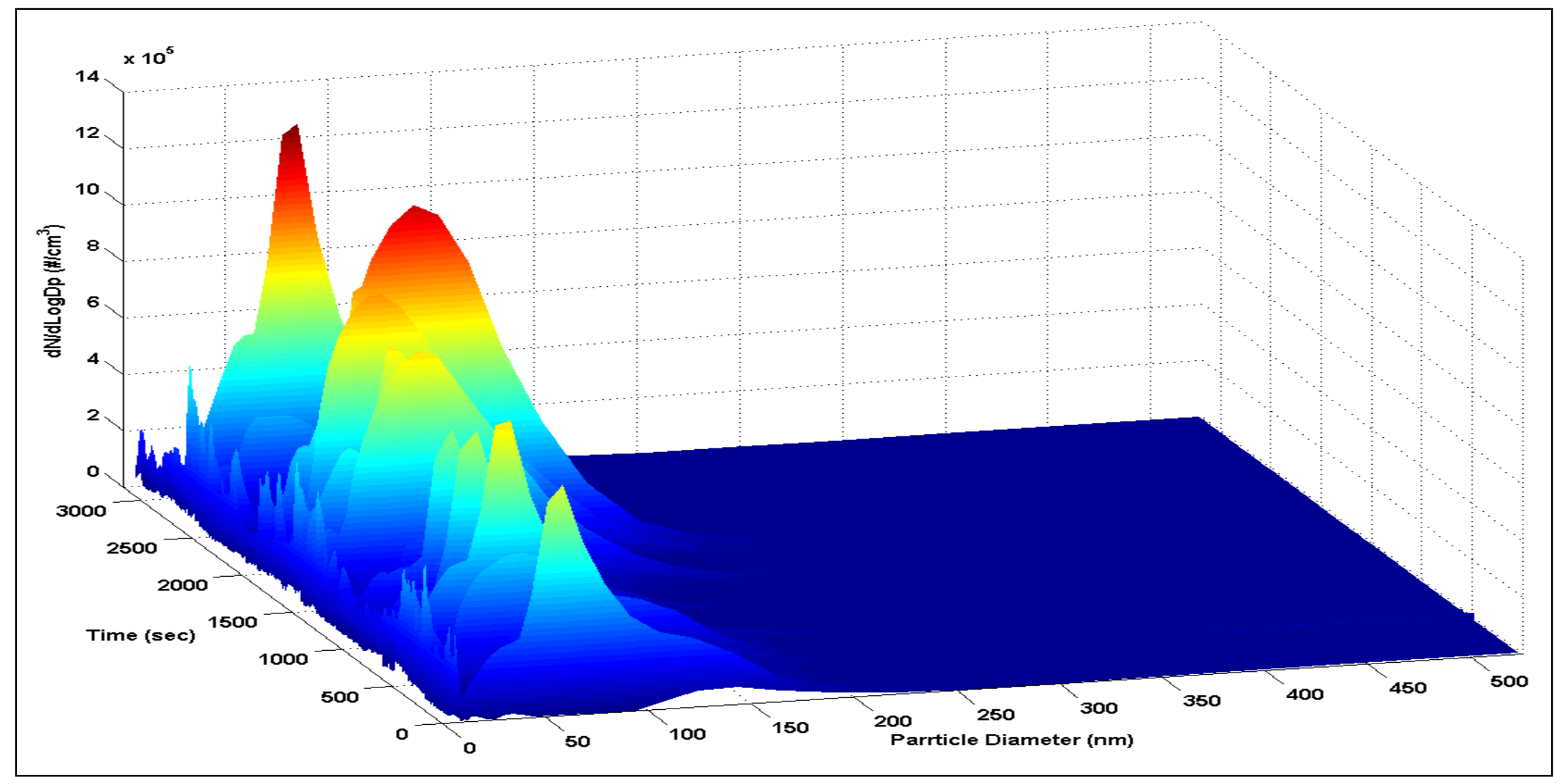

Bus 2 UDDS instantaneous particle size distribution measured with TSI EEPS (not corrected for dilution ratio)

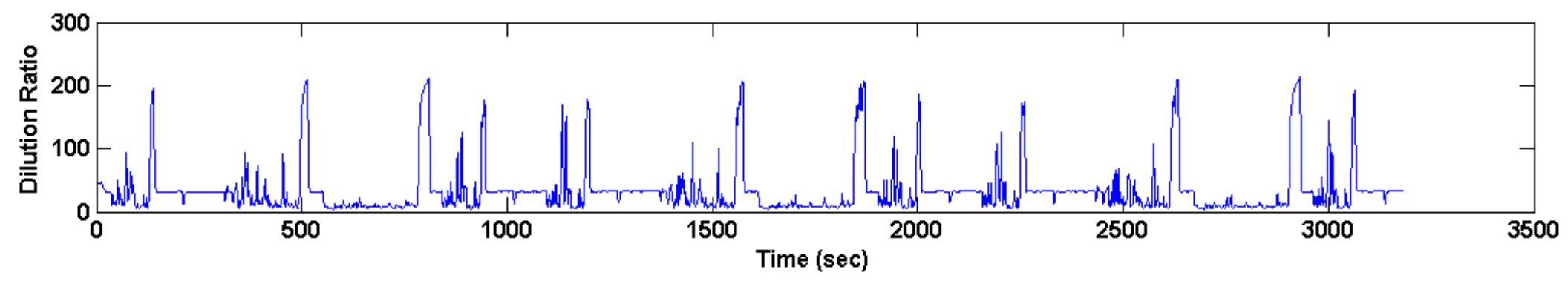

Bus 2 UDDS average instantaneous CVS dilution ratios 


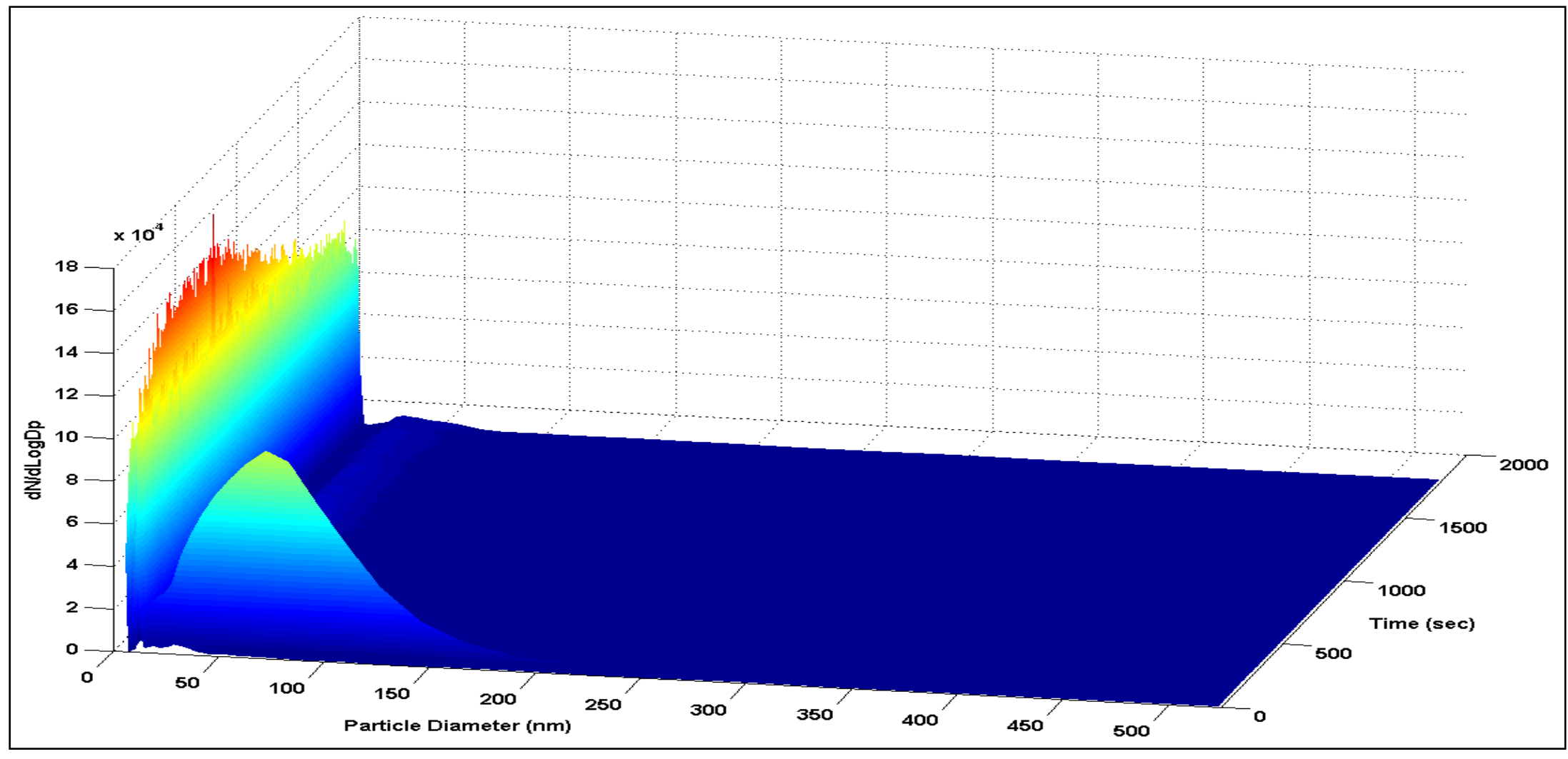

Bus 2 SS 45MPH instantaneous particle size distribution measured with TSI EEPS (not corrected for dilution ratio)

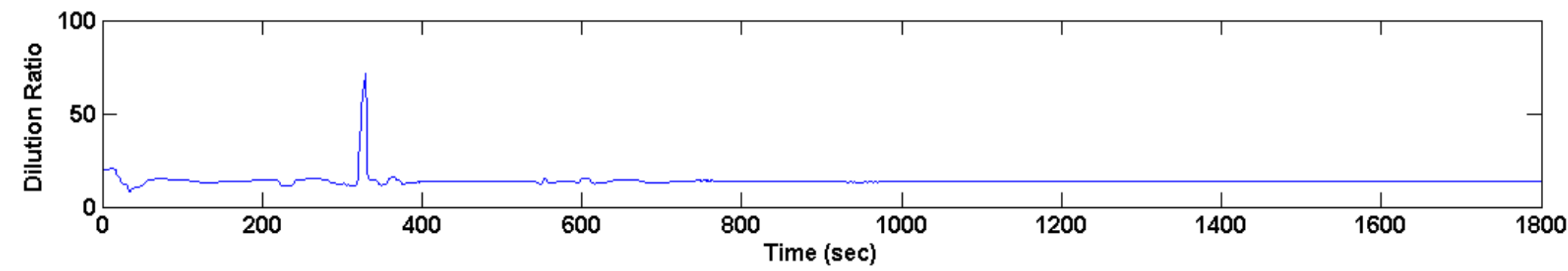

Bus 2 SS 45 MPH average instantaneous CVS dilution ratios 


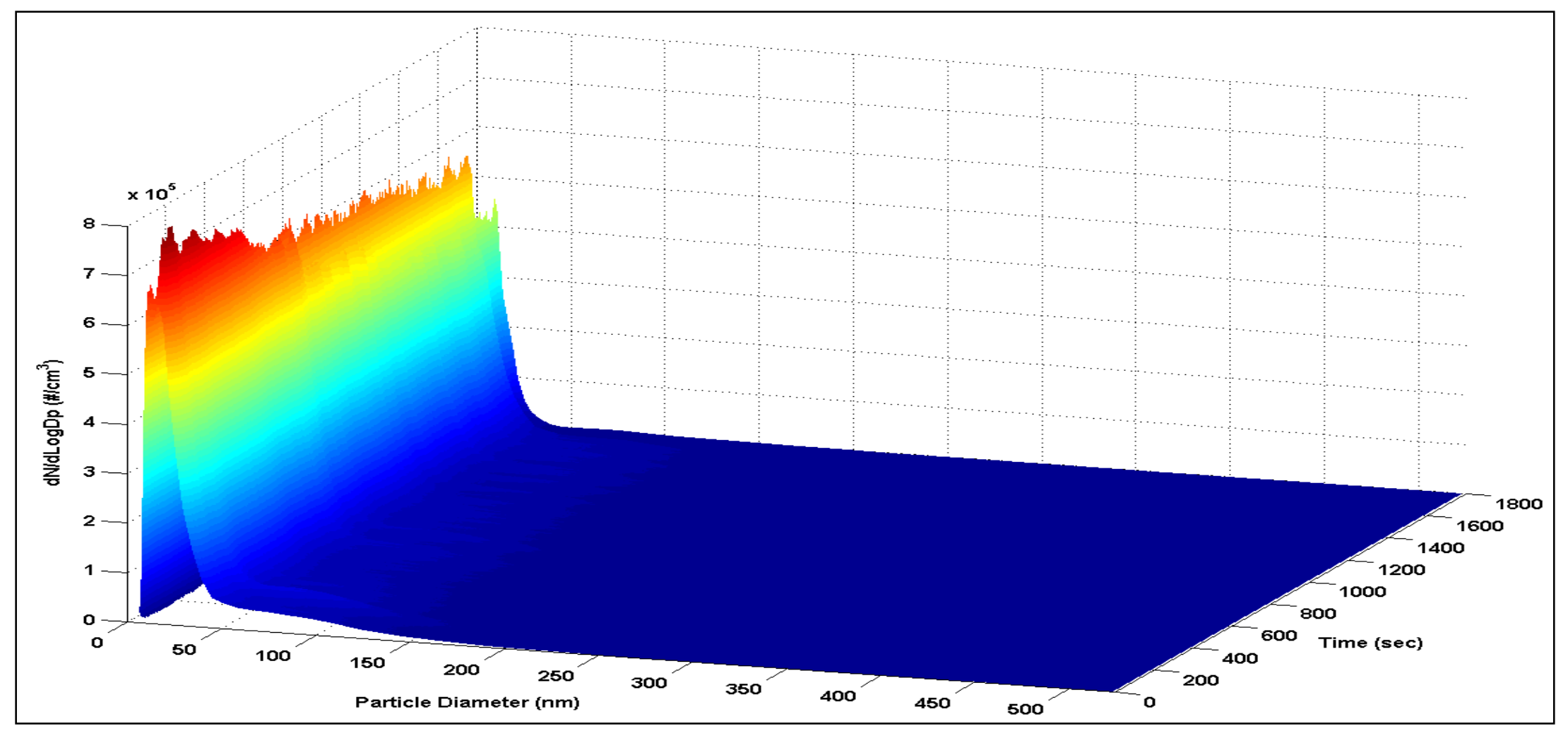

Bus 2 idle instantaneous particle size distribution measured with TSI EEPS (not corrected for dilution ratio)

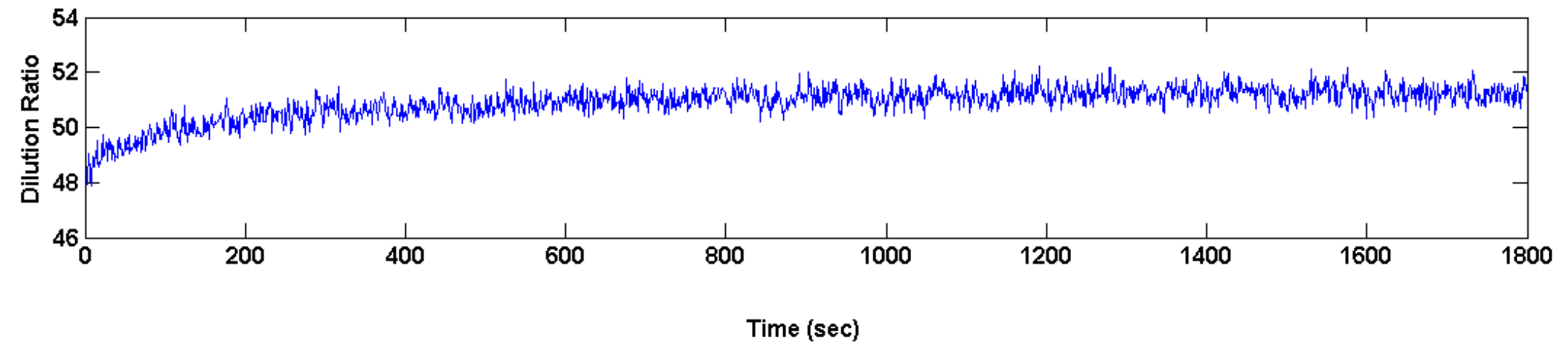

Bus 2 idle average instantaneous CVS dilution ratios

172 | P a g e 


\section{APPENDIX II- EC AND OC ANALYSIS RESULTS}

Detection limit: $0.8 \mathrm{mg} \mathrm{C} / \mathrm{cm}^{2}$; Reporting limit based on a quartz filter exposure area of $11.34 \mathrm{~cm}^{2}$.

\begin{tabular}{|c|c|c|c|c|c|c|}
\hline & Compound & $\begin{array}{c}\text { Carbon } \\
\text { Result } \\
\mu \mathrm{g} \mathrm{C/ \textrm {cm } ^ { 2 }} \\
\end{array}$ & $\begin{array}{c}\text { Carbon } \\
\text { Result } \\
\mu \mathrm{g} \mathrm{C} \\
\end{array}$ & $\begin{array}{c}\text { Carbon } \\
\text { Result } \\
\mu \mathrm{g} \mathrm{Clscf}\end{array}$ & $\begin{array}{l}\text { Total Carbon } \\
\text { Result mg C }\end{array}$ & $\begin{array}{c}\text { Total Carbon Result } \\
\text { mg C/mile \& mg } \\
\text { C/sec }\end{array}$ \\
\hline \multirow{15}{*}{ 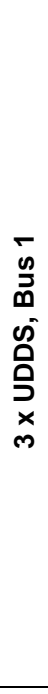 } & Organic Carbon 1 & $<.80$ & $<9.072$ & & & $\mathrm{BDL}$ \\
\hline & Organic Carbon 2 & 1.5 & 17.01 & 0.047 & 11.798 & 0.237 \\
\hline & Organic Carbon 3 & 1.6 & 18.144 & 0.050 & 12.585 & 0.252 \\
\hline & Organic Carbon 4 & $<.80$ & $<9.072$ & & & $\mathrm{BDL}$ \\
\hline & Elemental Carbon 1 & $<.80$ & $<9.072$ & & & $\mathrm{BDL}$ \\
\hline & Elemental Carbon 2 & $<.80$ & $<9.072$ & & & $\mathrm{BDL}$ \\
\hline & Elemental Carbon 3 & $<.80$ & $<9.072$ & & & $\mathrm{BDL}$ \\
\hline & Reflectance Pyrolyzed Organic Carbon & $<.80$ & $<9.072$ & & & $\mathrm{BDL}$ \\
\hline & Reflectance Organic Carbon & 4.1 & 46.494 & 0.128 & 32.249 & 0.647 \\
\hline & Reflectance Elemental Carbon & $<.80$ & $<9.072$ & & & $\mathrm{BDL}$ \\
\hline & Reflectance Total Carbon & 4.3 & 48.762 & 0.134 & 33.822 & 0.678 \\
\hline & Transmittance Pyrolyzed Organic Carbon & $<.80$ & $<9.072$ & & & BDL \\
\hline & Transmittance Organic Carbon & 4.3 & 48.762 & 0.134 & 33.822 & 0.678 \\
\hline & Transmittance Elemental Carbon & 4.0 & 45.36 & 0.125 & 31.462 & 0.631 \\
\hline & Transmittance Total Carbon & 4.3 & 48.762 & 0.134 & 33.822 & 0.678 \\
\hline \multirow{15}{*}{ 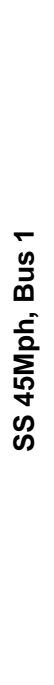 } & Organic Carbon 1 & $<.80$ & $<9.072$ & & & $\mathrm{BDL}$ \\
\hline & Organic Carbon 2 & 1.9 & 21.546 & 0.049 & 14.089 & 0.104 \\
\hline & Organic Carbon 3 & 1.3 & 14.742 & 0.034 & 9.640 & 0.071 \\
\hline & Organic Carbon 4 & $<.80$ & $<9.072$ & & & $\mathrm{BDL}$ \\
\hline & Elemental Carbon 1 & $<.80$ & $<9.072$ & & & $\mathrm{BDL}$ \\
\hline & Elemental Carbon 2 & $<.80$ & $<9.072$ & & & $\mathrm{BDL}$ \\
\hline & Elemental Carbon 3 & $<.80$ & $<9.072$ & & & $\mathrm{BDL}$ \\
\hline & Reflectance Pyrolyzed Organic Carbon & $<.80$ & $<9.072$ & & & $\mathrm{BDL}$ \\
\hline & Reflectance Organic Carbon & 3.7 & 41.958 & 0.096 & 27.437 & 0.203 \\
\hline & Reflectance Elemental Carbon & $<.80$ & $<9.072$ & & & $\mathrm{BDL}$ \\
\hline & Reflectance Total Carbon & 3.7 & 41.958 & 0.096 & 27.437 & 0.203 \\
\hline & Transmittance Pyrolyzed Organic Carbon & $<.80$ & $<9.072$ & & & $\mathrm{BDL}$ \\
\hline & Transmittance Organic Carbon & 3.7 & 41.958 & 0.096 & 27.437 & 0.203 \\
\hline & Transmittance Elemental Carbon & $<.80$ & $<9.072$ & & & $\mathrm{BDL}$ \\
\hline & Transmittance Total Carbon & 3.7 & 41.958 & 0.096 & 27.437 & 0.203 \\
\hline
\end{tabular}

173 | P a g e 
APPENDIX II CONTD.

\begin{tabular}{|c|c|c|c|c|c|c|}
\hline \multirow{11}{*}{ 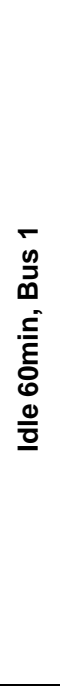 } & Organic Carbon 1 & $<.80$ & $<9.072$ & & & $\mathrm{BDL}$ \\
\hline & Organic Carbon 2 & 2.2 & 24.948 & 0.055 & 12.749 & 0.001 \\
\hline & Organic Carbon 3 & 1.6 & 18.144 & 0.040 & 9.272 & 0.001 \\
\hline & Elemental Carbon 1 & $<.80$ & $<9.072$ & & & $\mathrm{BDL}$ \\
\hline & Elemental Carbon 2 & $<.80$ & $<9.072$ & & & $\mathrm{BDL}$ \\
\hline & Elemental Carbon 3 & $<.80$ & $<9.072$ & & & $\mathrm{BDL}$ \\
\hline & Reflectance Organic Carbon & 4.2 & 47.628 & 0.106 & 24.340 & 0.002 \\
\hline & Reflectance Elemental Carbon & $<.80$ & $<9.072$ & & & $\mathrm{BDL}$ \\
\hline & Reflectance Total Carbon & 4.3 & 48.762 & 0.108 & 24.919 & 0.002 \\
\hline & Transmittance Pyrolyzed Organic Carbon & $<.80$ & $<9.072$ & & & $\mathrm{BDL}$ \\
\hline & Transmittance Organic Carbon & 4.3 & 48.762 & 0.108 & 24.919 & 0.002 \\
\hline \multirow{10}{*}{ 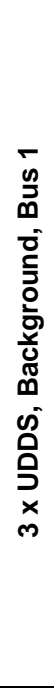 } & Organic Carbon 2 & $<.80$ & $<9.072$ & & & \\
\hline & Organic Carbon 3 & 1.0 & 11.34 & 0.094 & 7.866 & 0.002 \\
\hline & Organic Carbon 4 & $<.80$ & $<9.072$ & & & \\
\hline & Elemental Carbon 1 & $<.80$ & $<9.072$ & & & \\
\hline & Elemental Carbon 2 & $<.80$ & $<9.072$ & & & \\
\hline & Elemental Carbon 3 & $<.80$ & $<9.072$ & & & \\
\hline & Reflectance Pyrolyzed Organic Carbon & $<.80$ & $<9.072$ & & & \\
\hline & Reflectance Organic Carbon & 2.1 & 23.814 & 0.197 & 16.519 & 0.005 \\
\hline & Reflectance Elemental Carbon & $<.80$ & $<9.072$ & & & \\
\hline & Reflectance Total Carbon & 2.3 & 26.082 & 0.216 & 18.092 & 0.006 \\
\hline
\end{tabular}

174 | P a g e 
APPENDIX II CONTD.

\begin{tabular}{|c|c|c|c|c|c|c|}
\hline \multirow{15}{*}{ 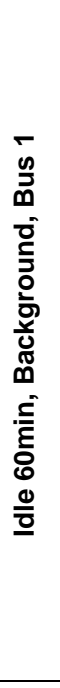 } & Organic Carbon 1 & $<.80$ & $<9.072$ & & & \\
\hline & Organic Carbon 2 & 1.2 & 13.608 & 0.091 & 6.941 & 0.002 \\
\hline & Organic Carbon 3 & 0.85 & 9.639 & 0.064 & 4.917 & 0.001 \\
\hline & Organic Carbon 4 & $<.80$ & $<9.072$ & & & \\
\hline & Elemental Carbon 1 & $<.80$ & $<9.072$ & & & \\
\hline & Elemental Carbon 2 & $<.80$ & $<9.072$ & & & \\
\hline & Elemental Carbon 3 & $<.80$ & $<9.072$ & & & \\
\hline & Reflectance Pyrolyzed Organic Carbon & $<.80$ & $<9.072$ & & & \\
\hline & Reflectance Organic Carbon & 2.3 & 26.082 & 0.174 & 13.304 & 0.004 \\
\hline & Reflectance Elemental Carbon & $<.80$ & $<9.072$ & & & \\
\hline & Reflectance Total Carbon & 2.3 & 26.082 & 0.174 & 13.304 & 0.004 \\
\hline & Transmittance Pyrolyzed Organic Carbon & $<.80$ & $<9.072$ & & & \\
\hline & Transmittance Organic Carbon & 2.3 & 26.082 & 0.174 & 13.304 & 0.004 \\
\hline & Transmittance Elemental Carbon & $<.80$ & $<9.072$ & & & \\
\hline & Transmittance Total Carbon & 2.3 & 26.082 & 0.174 & 13.304 & 0.004 \\
\hline \multirow{15}{*}{ 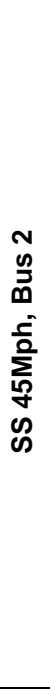 } & Organic Carbon 1 & $<.80$ & $<9.072$ & & & 0.000 \\
\hline & Organic Carbon 2 & 1.1 & 12.474 & 0.036 & 8.266 & 0.076 \\
\hline & Organic Carbon 3 & 0.87 & 9.8658 & 0.028 & 6.537 & 0.060 \\
\hline & Organic Carbon 4 & $<.80$ & $<9.072$ & & & 0.000 \\
\hline & Elemental Carbon 1 & $<.80$ & $<9.072$ & & & 0.000 \\
\hline & Elemental Carbon 2 & $<.80$ & $<9.072$ & & & 0.000 \\
\hline & Elemental Carbon 3 & $<.80$ & $<9.072$ & & & 0.000 \\
\hline & Reflectance Pyrolyzed Organic Carbon & $<.80$ & $<9.072$ & & & 0.000 \\
\hline & Reflectance Organic Carbon & 2.3 & 26.082 & 0.075 & 17.283 & 0.159 \\
\hline & Reflectance Elemental Carbon & $<.80$ & $<9.072$ & & & 0.000 \\
\hline & Reflectance Total Carbon & 2.4 & 27.216 & 0.078 & 18.034 & 0.166 \\
\hline & Transmittance Pyrolyzed Organic Carbon & $<.80$ & $<9.072$ & & & 0.000 \\
\hline & Transmittance Organic Carbon & 2.4 & 27.216 & 0.078 & 18.034 & 0.166 \\
\hline & Transmittance Elemental Carbon & $<.80$ & $<9.072$ & & & 0.000 \\
\hline & Transmittance Total Carbon & 2.4 & 27.216 & 0.078 & 18.034 & 0.166 \\
\hline
\end{tabular}

175 | P a g e 
APPENDIX II CONTD.

\begin{tabular}{|c|c|c|c|c|c|c|}
\hline \multirow{15}{*}{ 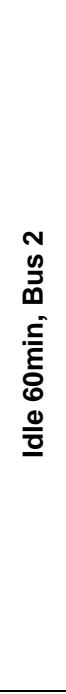 } & Organic Carbon 1 & $<.80$ & $<9.072$ & & & \\
\hline & Organic Carbon 2 & 1.4 & 15.876 & 0.038 & 10.837 & 0.001 \\
\hline & Organic Carbon 3 & 2.5 & 28.35 & 0.068 & 19.351 & 0.002 \\
\hline & Organic Carbon 4 & $<.80$ & $<9.072$ & & & \\
\hline & Elemental Carbon 1 & 3.8 & 43.092 & 0.103 & 29.414 & 0.003 \\
\hline & Elemental Carbon 2 & 3.6 & 40.824 & 0.098 & 27.866 & 0.003 \\
\hline & Elemental Carbon 3 & $<.80$ & $<9.072$ & & & \\
\hline & Reflectance Pyrolyzed Organic Carbon & $<.80$ & $<9.072$ & & & \\
\hline & Reflectance Organic Carbon & 5.5 & 62.37 & 0.149 & 42.573 & 0.004 \\
\hline & Reflectance Elemental Carbon & 7.0 & 79.38 & 0.190 & 54.183 & 0.005 \\
\hline & Reflectance Total Carbon & 12 & 136.08 & 0.326 & 92.886 & 0.009 \\
\hline & Transmittance Pyrolyzed Organic Carbon & 1.0 & 11.34 & 0.027 & 7.740 & 0.001 \\
\hline & Transmittance Organic Carbon & 5.9 & 66.906 & 0.160 & 45.669 & 0.004 \\
\hline & Transmittance Elemental Carbon & 6.5 & 73.71 & 0.177 & 50.313 & 0.005 \\
\hline & Transmittance Total Carbon & 12 & 136.08 & 0.326 & 92.886 & 0.009 \\
\hline \multirow{15}{*}{ 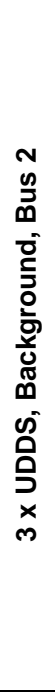 } & Organic Carbon 1 & $<.80$ & $<9.072$ & & & \\
\hline & Organic Carbon 2 & 1.0 & 11.34 & 0.090 & 7.445 & 0.002 \\
\hline & Organic Carbon 3 & 1.2 & 13.608 & 0.107 & 8.934 & 0.003 \\
\hline & Organic Carbon 4 & $<.80$ & $<9.072$ & & & \\
\hline & Elemental Carbon 1 & $<.80$ & $<9.072$ & & & \\
\hline & Elemental Carbon 2 & $<.80$ & $<9.072$ & & & \\
\hline & Elemental Carbon 3 & $<.80$ & $<9.072$ & & & \\
\hline & Reflectance Pyrolyzed Organic Carbon & $<.80$ & $<9.072$ & & & \\
\hline & Reflectance Organic Carbon & 2.7 & 30.618 & 0.242 & 20.102 & 0.006 \\
\hline & Reflectance Elemental Carbon & $<.80$ & $<9.072$ & & & \\
\hline & Reflectance Total Carbon & 3.0 & 34.02 & 0.269 & 22.336 & 0.007 \\
\hline & Transmittance Pyrolyzed Organic Carbon & $<.80$ & $<9.072$ & & & \\
\hline & Transmittance Organic Carbon & 3.0 & 34.02 & 0.269 & 22.336 & 0.007 \\
\hline & Transmittance Elemental Carbon & $<.80$ & $<9.072$ & & & \\
\hline & Transmittance Total Carbon & 3.0 & 34.02 & 0.269 & 22.336 & 0.007 \\
\hline
\end{tabular}

176 | P a g e 
APPENDIX II CONTD.

\begin{tabular}{|c|c|c|c|c|c|c|}
\hline \multirow{15}{*}{ 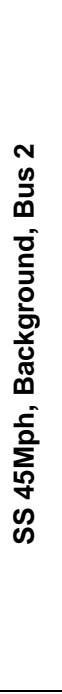 } & Organic Carbon 1 & $<.80$ & $<9.072$ & & & \\
\hline & Organic Carbon 2 & $<.80$ & $<9.072$ & & & \\
\hline & Organic Carbon 3 & 0.88 & 9.9792 & 0.070 & 6.547 & 0.002 \\
\hline & Organic Carbon 4 & $<.80$ & $<9.072$ & & & \\
\hline & Elemental Carbon 1 & $<.80$ & $<9.072$ & & & \\
\hline & Elemental Carbon 2 & $<.80$ & $<9.072$ & & & \\
\hline & Elemental Carbon 3 & $<.80$ & $<9.072$ & & & \\
\hline & Reflectance Pyrolyzed Organic Carbon & $<.80$ & $<9.072$ & & & \\
\hline & Reflectance Organic Carbon & 2.1 & 23.814 & 0.166 & 15.623 & 0.004 \\
\hline & Reflectance Elemental Carbon & $<.80$ & $<9.072$ & & & \\
\hline & Reflectance Total Carbon & 2.3 & 26.082 & 0.182 & 17.110 & 0.005 \\
\hline & Transmittance Pyrolyzed Organic Carbon & $<.80$ & $<9.072$ & & & \\
\hline & Transmittance Organic Carbon & 2.3 & 26.082 & 0.182 & 17.110 & 0.005 \\
\hline & Transmittance Elemental Carbon & $<.80$ & $<9.072$ & & & \\
\hline & Transmittance Total Carbon & 2.3 & 26.082 & 0.182 & 17.110 & 0.005 \\
\hline \multirow{15}{*}{ 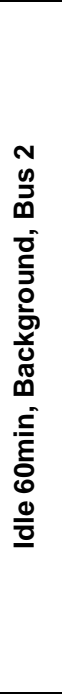 } & Organic Carbon 1 & $<.80$ & $<9.072$ & & & \\
\hline & Organic Carbon 2 & 1.2 & 13.608 & 0.099 & 9.437 & 0.003 \\
\hline & Organic Carbon 3 & 1.5 & 17.01 & 0.124 & 11.797 & 0.003 \\
\hline & Organic Carbon 4 & $<.80$ & $<9.072$ & & & \\
\hline & Elemental Carbon 1 & $<.80$ & $<9.072$ & & & \\
\hline & Elemental Carbon 2 & $<.80$ & $<9.072$ & & & \\
\hline & Elemental Carbon 3 & $<.80$ & $<9.072$ & & & \\
\hline & Reflectance Pyrolyzed Organic Carbon & $<.80$ & $<9.072$ & & & \\
\hline & Reflectance Organic Carbon & 3.4 & 38.556 & 0.281 & 26.739 & 0.007 \\
\hline & Reflectance Elemental Carbon & $<.80$ & $<9.072$ & & & \\
\hline & Reflectance Total Carbon & 3.7 & 41.958 & 0.306 & 29.098 & 0.008 \\
\hline & Transmittance Pyrolyzed Organic Carbon & $<.80$ & $<9.072$ & & & \\
\hline & Transmittance Organic Carbon & 3.4 & 38.556 & 0.281 & 26.739 & 0.007 \\
\hline & Transmittance Elemental Carbon & $<.80$ & $<9.072$ & & & \\
\hline & Transmittance Total Carbon & 3.7 & 41.958 & 0.306 & 29.098 & 0.008 \\
\hline
\end{tabular}

177 | P a g e 


\section{APPENDIX III- ICP-MS ANALYSIS RESULTS OF METALS AND ELEMENTS}

Water extracted from filter and analyses performed using magnetic-sector ICP-MS (Thermo-Finnigan Element 2)

\begin{tabular}{|c|c|c|c|c|c|}
\hline & $\begin{array}{c}\text { Bus 1 3X } \\
\text { UDDS } \\
\text { (ng/filter) }\end{array}$ & $\begin{array}{c}\text { Bus 1 60 min } \\
\text { IDLE (ng/filter) }\end{array}$ & $\begin{array}{c}\text { Bus 1 60 min } \\
\text { IDLE (ng/filter) }\end{array}$ & $\begin{array}{c}\text { Bus1 SS 45 } \\
\text { MPH } \\
\text { (ng/filter) }\end{array}$ & $\begin{array}{c}\text { Bus 1 SS 45 } \\
\text { MPH } \\
\text { (ng/filter) }\end{array}$ \\
\hline $\mathbf{L i}$ & $5.407 \mathrm{E}-01$ & $7.861 \mathrm{E}-02$ & $1.090 \mathrm{E}-01$ & $3.827 \mathrm{E}-02$ & $9.641 \mathrm{E}-02$ \\
\hline $\mathbf{B}$ & $3.070 \mathrm{E}+00$ & $6.973 \mathrm{E}-01$ & $4.412 \mathrm{E}+00$ & $3.907 \mathrm{E}+00$ & $5.809 \mathrm{E}+00$ \\
\hline $\mathbf{N a}$ & $3.784 \mathrm{E}+02$ & $1.100 \mathrm{E}+02$ & $1.101 \mathrm{E}+02$ & $8.359 \mathrm{E}+02$ & $1.659 \mathrm{E}+02$ \\
\hline $\mathbf{M g}$ & $8.035 \mathrm{E}+01$ & $4.770 \mathrm{E}+01$ & $1.547 \mathrm{E}+02$ & $4.862 \mathrm{E}+01$ & $8.645 \mathrm{E}+01$ \\
\hline $\mathbf{A l}$ & $1.831 \mathrm{E}+01$ & $1.747 \mathrm{E}+01$ & $1.859 \mathrm{E}+01$ & $5.858 \mathrm{E}+00$ & $1.504 \mathrm{E}+01$ \\
\hline $\mathbf{P}$ & $7.240 \mathrm{E}+00$ & $2.663 \mathrm{E}+00$ & $4.944 \mathrm{E}+00$ & $5.215 \mathrm{E}+00$ & $3.819 \mathrm{E}+00$ \\
\hline $\mathbf{S}$ & $1.185 \mathrm{E}+02$ & $2.767 \mathrm{E}+01$ & $4.433 \mathrm{E}+01$ & $6.738 \mathrm{E}+01$ & $1.015 \mathrm{E}+02$ \\
\hline $\mathbf{K}$ & $6.587 \mathrm{E}+01$ & $4.137 \mathrm{E}+01$ & $5.639 \mathrm{E}+01$ & $4.259 \mathrm{E}+01$ & $5.919 \mathrm{E}+01$ \\
\hline $\mathbf{C a}$ & $9.125 \mathrm{E}+02$ & $5.260 \mathrm{E}+02$ & $6.709 \mathrm{E}+02$ & $6.986 \mathrm{E}+02$ & $8.587 \mathrm{E}+02$ \\
\hline $\mathbf{T i}$ & $5.438 \mathrm{E}-02$ & $1.117 \mathrm{E}-01$ & $2.086 \mathrm{E}-01$ & $3.497 \mathrm{E}-02$ & $1.916 \mathrm{E}-02$ \\
\hline $\mathbf{V}$ & $5.078 \mathrm{E}-02$ & $2.096 \mathrm{E}-02$ & $4.782 \mathrm{E}-02$ & $7.157 \mathrm{E}-02$ & $4.527 \mathrm{E}-02$ \\
\hline $\mathbf{C r}$ & $6.414 \mathrm{E}-01$ & $2.164 \mathrm{E}-01$ & $1.177 \mathrm{E}+00$ & $3.628 \mathrm{E}+00$ & $4.065 \mathrm{E}+00$ \\
\hline $\mathbf{M n}$ & $6.683 \mathrm{E}+00$ & $7.282 \mathrm{E}-01$ & $1.327 \mathrm{E}+00$ & $1.009 \mathrm{E}+00$ & $1.255 \mathrm{E}+00$ \\
\hline $\mathbf{F e}$ & $6.306 \mathrm{E}+00$ & $5.315 \mathrm{E}+00$ & $4.497 \mathrm{E}+00$ & $3.612 \mathrm{E}+01$ & $1.862 \mathrm{E}+01$ \\
\hline $\mathbf{C o}$ & $5.434 \mathrm{E}-02$ & $6.541 \mathrm{E}-02$ & $9.177 \mathrm{E}-02$ & $9.572 \mathrm{E}-02$ & $1.326 \mathrm{E}-01$ \\
\hline $\mathbf{N i}$ & $2.207 \mathrm{E}+00$ & $3.611 \mathrm{E}-01$ & $9.734 \mathrm{E}-01$ & $2.067 \mathrm{E}+00$ & $2.527 \mathrm{E}+00$ \\
\hline $\mathbf{C u}$ & $4.430 \mathrm{E}+00$ & $7.837 \mathrm{E}-01$ & $1.958 \mathrm{E}+00$ & $8.145 \mathrm{E}-01$ & $3.088 \mathrm{E}+00$ \\
\hline $\mathbf{Z n}$ & $2.152 \mathrm{E}+01$ & $1.019 \mathrm{E}+01$ & $1.592 \mathrm{E}+01$ & $1.605 \mathrm{E}+01$ & $1.549 \mathrm{E}+01$ \\
\hline $\mathbf{M o}$ & $2.288 \mathrm{E}-01$ & $7.258 \mathrm{E}-02$ & $1.314 \mathrm{E}-01$ & $2.205 \mathrm{E}-01$ & $2.573 \mathrm{E}-01$ \\
\hline $\mathbf{R h}$ & $3.578 \mathrm{E}-04$ & $2.739 \mathrm{E}-04$ & $4.187 \mathrm{E}-04$ & $7.605 \mathrm{E}-04$ & $1.583 \mathrm{E}-03$ \\
\hline $\mathbf{P d}$ & $1.498 \mathrm{E}-02$ & $1.214 \mathrm{E}-02$ & $1.010 \mathrm{E}-02$ & $6.580 \mathrm{E}-03$ & $1.535 \mathrm{E}-02$ \\
\hline $\mathbf{C d}$ & $5.757 \mathrm{E}-02$ & $4.001 \mathrm{E}-02$ & $1.416 \mathrm{E}-01$ & $3.328 \mathrm{E}-02$ & $5.871 \mathrm{E}-02$ \\
\hline $\mathbf{P t}$ & $-1.150 \mathrm{E}-04$ & $1.441 \mathrm{E}-03$ & $2.318 \mathrm{E}-03$ & $8.121 \mathrm{E}-04$ & $1.160 \mathrm{E}-03$ \\
\hline & & & & & \\
\hline
\end{tabular}


APPENDIX III CONTD.

\begin{tabular}{|c|c|c|c|c|c|c|}
\hline & $\begin{array}{c}\text { Bus } 23 X \\
\text { UDDS } \\
\text { (ng/filter) }\end{array}$ & $\begin{array}{c}\text { Bus } 23 X \\
\text { UDDS } \\
\text { (ng/filter) }\end{array}$ & $\begin{array}{c}\text { Bus } 2 \text { SS } 45 \\
\text { MPH } \\
\text { (ng/filter) }\end{array}$ & $\begin{array}{c}\text { Bus } 2 \text { SS } 45 \\
\text { MPH } \\
\text { (ng/filter) }\end{array}$ & $\begin{array}{l}\text { Bus } 260 \mathrm{~min} \\
\text { Idle (ng/filter) }\end{array}$ & $\begin{array}{l}\text { Bus } 260 \mathrm{~min} \\
\text { Idle (ng/filter) }\end{array}$ \\
\hline $\mathbf{L i}$ & $2.696 \mathrm{E}-02$ & $1.206 \mathrm{E}-01$ & 5.097E-02 & $2.015 \mathrm{E}-02$ & $1.486 \mathrm{E}-03$ & $-4.301 \mathrm{E}-03$ \\
\hline B & $2.039 \mathrm{E}-01$ & $4.180 \mathrm{E}+00$ & $2.339 \mathrm{E}+00$ & 4.205E-01 & $9.778 \mathrm{E}-01$ & 4.453E-01 \\
\hline $\mathrm{Na}$ & $7.289 \mathrm{E}+00$ & $6.121 \mathrm{E}+01$ & $3.461 \mathrm{E}+01$ & $4.038 \mathrm{E}+01$ & $2.967 \mathrm{E}+01$ & $1.416 \mathrm{E}+02$ \\
\hline $\mathrm{Mg}$ & $4.597 \mathrm{E}+01$ & $4.004 \mathrm{E}+01$ & 4.193E+01 & $1.880 \mathrm{E}+01$ & $4.975 \mathrm{E}+01$ & $3.667 \mathrm{E}+01$ \\
\hline Al & $3.869 \mathrm{E}+00$ & 4.657E-01 & $8.110 \mathrm{E}+00$ & $1.026 \mathrm{E}+01$ & $9.719 \mathrm{E}+00$ & $5.542 \mathrm{E}+00$ \\
\hline $\mathbf{P}$ & $8.893 E+01$ & $2.531 \mathrm{E}+00$ & $8.110 \mathrm{E}+00$ & 7.311E-01 & $2.948 \mathrm{E}+01$ & $5.991 \mathrm{E}+01$ \\
\hline$S$ & $3.968 \mathrm{E}+01$ & $3.171 \mathrm{E}+01$ & $3.877 \mathrm{E}+01$ & $5.663 \mathrm{E}+00$ & $7.384 \mathrm{E}+01$ & $2.252 \mathrm{E}+01$ \\
\hline $\mathrm{K}$ & $8.185 E+00$ & $4.110 \mathrm{E}+01$ & $1.333 \mathrm{E}+01$ & $1.109 \mathrm{E}+02$ & $1.652 \mathrm{E}+01$ & $2.842 \mathrm{E}+01$ \\
\hline $\mathrm{Ca}$ & $9.310 \mathrm{E}+02$ & $6.921 \mathrm{E}+02$ & $6.672 \mathrm{E}+02$ & $3.108 \mathrm{E}+02$ & $6.875 \mathrm{E}+02$ & $7.750 \mathrm{E}+02$ \\
\hline Sc & $2.077 \mathrm{E}-03$ & $1.105 \mathrm{E}-03$ & $3.068 \mathrm{E}-03$ & $1.121 \mathrm{E}-03$ & 4.952E-03 & $-1.788 \mathrm{E}-03$ \\
\hline $\mathrm{Ti}$ & $2.092 \mathrm{E}-01$ & $9.177 \mathrm{E}-02$ & $3.680 \mathrm{E}-01$ & $5.446 \mathrm{E}-02$ & $1.083 \mathrm{E}-01$ & $1.440 \mathrm{E}-01$ \\
\hline $\mathbf{v}$ & 4.555E-02 & $2.450 \mathrm{E}-02$ & $9.860 \mathrm{E}-02$ & $1.646 \mathrm{E}-02$ & $3.898 \mathrm{E}-02$ & 7.131E-02 \\
\hline $\mathrm{Cr}$ & $5.884 \mathrm{E}-01$ & $6.924 \mathrm{E}-01$ & $4.902 \mathrm{E}+00$ & $2.239 \mathrm{E}-01$ & $6.216 \mathrm{E}-01$ & $1.158 \mathrm{E}+01$ \\
\hline $\mathrm{Mn}$ & $1.137 \mathrm{E}+00$ & $7.128 \mathrm{E}-01$ & $7.666 \mathrm{E}-01$ & 4.248E-01 & $7.856 \mathrm{E}-01$ & 7.504E-01 \\
\hline $\mathrm{Fe}$ & $9.560 \mathrm{E}+00$ & $3.434 \mathrm{E}+00$ & $3.038 \mathrm{E}+01$ & $1.848 \mathrm{E}+00$ & $4.312 \mathrm{E}+00$ & $2.131 \mathrm{E}+01$ \\
\hline Co & $5.320 \mathrm{E}+00$ & $6.234 \mathrm{E}-02$ & 8.105E-01 & $1.451 \mathrm{E}-01$ & $6.209 \mathrm{E}-02$ & $1.161 \mathrm{E}-01$ \\
\hline $\mathbf{N i}$ & $1.053 \mathrm{E}+00$ & 4.707E-01 & $2.172 \mathrm{E}+00$ & $3.995 \mathrm{E}-01$ & 6.017E-01 & $2.238 \mathrm{E}+00$ \\
\hline $\mathrm{Cu}$ & $1.871 \mathrm{E}+00$ & 4.843E-01 & 3.147E-01 & 4.723E-01 & $2.971 \mathrm{E}+00$ & $6.202 \mathrm{E}+00$ \\
\hline $\mathrm{Zn}$ & $9.711 \mathrm{E}+01$ & $2.126 \mathrm{E}+01$ & $1.155 \mathrm{E}+01$ & $9.531 \mathrm{E}+00$ & $2.082 \mathrm{E}+01$ & $4.502 \mathrm{E}+01$ \\
\hline Mo & 3.304E-01 & $3.642 \mathrm{E}-01$ & $2.671 \mathrm{E}-01$ & $3.486 \mathrm{E}-02$ & $1.381 \mathrm{E}-01$ & $5.874 \mathrm{E}-01$ \\
\hline $\mathbf{R h}$ & $1.265 \mathrm{E}-03$ & 1.207E-04 & 1.129E-04 & $9.029 \mathrm{E}-04$ & $6.975 \mathrm{E}-04$ & $1.709 \mathrm{E}-03$ \\
\hline Pd & $2.851 \mathrm{E}-03$ & $9.103 \mathrm{E}-03$ & $5.543 \mathrm{E}-03$ & $3.385 \mathrm{E}-03$ & 1.105E-02 & 2.277E-04 \\
\hline $\mathrm{Cd}$ & $6.370 \mathrm{E}-03$ & $1.917 \mathrm{E}-02$ & $4.436 \mathrm{E}-02$ & $3.932 \mathrm{E}-02$ & 4.634E-02 & $6.127 \mathrm{E}-02$ \\
\hline $\mathrm{Pt}$ & 5.299E-04 & $1.447 \mathrm{E}-03$ & $1.021 \mathrm{E}-04$ & $1.246 \mathrm{E}-03$ & $2.714 \mathrm{E}-03$ & $1.322 \mathrm{E}-03$ \\
\hline
\end{tabular}

179 | P a g e 


\section{APPENDIX III CONTD.}

\begin{tabular}{|c|c|c|c|c|c|c|}
\hline & $\begin{array}{l}\text { 3X UDDS } \\
\text { Background } \\
\text { (ng/filter) }\end{array}$ & $\begin{array}{l}60 \text { min Idle } \\
\text { Background } \\
\text { (ng/filter) }\end{array}$ & $\begin{array}{l}\text { SS } 45 \text { MPH } \\
\text { Background } \\
\text { (ng/filter) }\end{array}$ & $\begin{array}{l}\text { 3X UDDS } \\
\text { Background } \\
\text { (ng/filter) }\end{array}$ & $\begin{array}{l}\text { SS } 45 \text { MPH } \\
\text { Background } \\
\text { (ng/filter) }\end{array}$ & $\begin{array}{l}60 \text { min Idle } \\
\text { Background } \\
\text { (ng/filter) }\end{array}$ \\
\hline $\mathbf{L i}$ & 1.192E-01 & 6.737E-02 & 1.514E-02 & $1.931 \mathrm{E}-02$ & $3.858 \mathrm{E}-02$ & $2.784 \mathrm{E}-01$ \\
\hline B & $846 \mathrm{E}-01$ & 4.473E-01 & $.979 \mathrm{E}-01$ & $2.040 \mathrm{E}+00$ & $3.066 \mathrm{E}-01$ & $-9.148 \mathrm{E}-03$ \\
\hline $\mathrm{Na}$ & $7.806 \mathrm{E}+01$ & $5.604 \mathrm{E}+02$ & $9.040 \mathrm{E}+02$ & $3.157 \mathrm{E}+02$ & $3.653 \mathrm{E}+02$ & $4.342 E+02$ \\
\hline $\mathrm{Mg}$ & $5.881 E+01$ & $4.089 E+01$ & $4.287 E+01$ & $1.243 E+02$ & $8.569 E+01$ & $5.996 \mathrm{E}+01$ \\
\hline Al & $9.598 \mathrm{E}+00$ & $1.899 \mathrm{E}+01$ & $6.310 \mathrm{E}+00$ & $1.496 \mathrm{E}+01$ & $5.959 \mathrm{E}+00$ & $1.810 \mathrm{E}+01$ \\
\hline $\mathbf{P}$ & $4.311 E+00$ & $2.801 \mathrm{E}+00$ & $1.903 \mathrm{E}+00$ & $1.378 \mathrm{E}+00$ & $7.137 E+00$ & $2.808 \mathrm{E}+00$ \\
\hline$S$ & $4.978 \mathrm{E}+01$ & $6.009 \mathrm{E}+01$ & $1.212 \mathrm{E}+01$ & $1.059 \mathrm{E}+01$ & $1.633 \mathrm{E}+01$ & $6.079 E+01$ \\
\hline$K$ & $1.982 \mathrm{E}+01$ & $2.354 \mathrm{E}+01$ & $9.721 \mathrm{E}+00$ & $2.432 \mathrm{E}+01$ & $3.003 E+01$ & $1.286 \mathrm{E}+02$ \\
\hline $\mathrm{Ca}$ & $6.702 \mathrm{E}+02$ & $6.318 \mathrm{E}+02$ & $7.080 \mathrm{E}+02$ & $1.059 \mathrm{E}+03$ & $1.063 \mathrm{E}+03$ & $1.189 \mathrm{E}+03$ \\
\hline Sc & 8.919E-03 & $1.271 \mathrm{E}-04$ & $4.279 \mathrm{E}-03$ & $4.282 \mathrm{E}-03$ & $5.864 \mathrm{E}-03$ & $-7.703 E-04$ \\
\hline $\mathrm{Ti}$ & $3.331 E-02$ & $9.292 \mathrm{E}-02$ & $-4.212 \mathrm{E}-03$ & 5.449E-01 & $2.275 \mathrm{E}-01$ & $-4.774 \mathrm{E}-02$ \\
\hline $\mathbf{v}$ & $6.115 \mathrm{E}-02$ & 8.141E-03 & 7.439E-03 & $3.671 \mathrm{E}-02$ & & 1.736E-02 \\
\hline $\mathrm{Cr}$ & 4.571E-01 & $1.732 \mathrm{E}-01$ & $4.032 \mathrm{E}-01$ & $2.136 \mathrm{E}-01$ & $2.107 E-01$ & $4.125 \mathrm{E}-01$ \\
\hline $\mathrm{Mn}$ & $2.809 \mathrm{E}-01$ & 4.015E-01 & $2.369 \mathrm{E}-01$ & $1.774 \mathrm{E}+00$ & $7.888 \mathrm{E}-01$ & $1.161 \mathrm{E}+00$ \\
\hline $\mathrm{Fe}$ & $3.915 E+00$ & $1.366 \mathrm{E}+00$ & $3.359 \mathrm{E}+00$ & $5.598 \mathrm{E}+00$ & $3.972 \mathrm{E}+00$ & $3.612 E+00$ \\
\hline Co & $2.021 \mathrm{E}-01$ & $1.587 \mathrm{E}-02$ & $2.183 \mathrm{E}-02$ & $3.564 \mathrm{E}-02$ & $3.078 \mathrm{E}-02$ & $8.878 \mathrm{E}-02$ \\
\hline $\mathrm{Ni}$ & 1.707E-01 & $1.739 \mathrm{E}-01$ & $2.014 \mathrm{E}-01$ & $5.741 \mathrm{E}-01$ & $2.526 \mathrm{E}-01$ & $2.091 \mathrm{E}+00$ \\
\hline $\mathrm{Cu}$ & $1.321 \mathrm{E}+00$ & $1.814 \mathrm{E}-03$ & $3.224 \mathrm{E}-01$ & $1.540 \mathrm{E}-01$ & $3.822 \mathrm{E}-01$ & 6.647E-01 \\
\hline $\mathrm{Zn}$ & $1.414 \mathrm{E}+01$ & $9.562 E+00$ & $7.840 \mathrm{E}+00$ & $1.001 \mathrm{E}+01$ & $8.113 E+00$ & $4.824 \mathrm{E}+01$ \\
\hline Mo & $1.613 \mathrm{E}-01$ & $7.546 \mathrm{E}-02$ & $4.116 \mathrm{E}-02$ & $1.295 \mathrm{E}-01$ & $2.163 \mathrm{E}-01$ & $1.312 \mathrm{E}-01$ \\
\hline $\mathbf{R h}$ & 1.429E-03 & 4.442E-04 & $1.678 \mathrm{E}-03$ & $7.256 \mathrm{E}-04$ & $1.838 \mathrm{E}-04$ & $3.408 \mathrm{E}-04$ \\
\hline Pd & $1.753 \mathrm{E}-02$ & $1.231 \mathrm{E}-02$ & $3.312 \mathrm{E}-03$ & $8.078 \mathrm{E}-03$ & $1.043 \mathrm{E}-02$ & $8.223 \mathrm{E}-03$ \\
\hline $\mathrm{Cd}$ & $2.190 \mathrm{E}-02$ & $2.941 \mathrm{E}-02$ & $9.763 \mathrm{E}-03$ & $1.909 \mathrm{E}-02$ & $7.008 \mathrm{E}-02$ & 7.501E-02 \\
\hline Pt & $1.440 \mathrm{E}-03$ & $1.253 \mathrm{E}-03$ & $1.552 \mathrm{E}-03$ & 8.904E-04 & $5.558 \mathrm{E}-04$ & $1.641 \mathrm{E}-03$ \\
\hline
\end{tabular}

180 | P a g e 


\section{APPENDIX IV- CARBONYL RESULTS}

\section{Value of zero indicates analyte concentration below the detection limit of $0.007 \mathrm{mg} / \mathrm{L}$ of the extract. $4.4 \mathrm{ml}$ of the extract was used for analysis}

\begin{tabular}{|c|c|c|c|c|c|}
\hline & \multicolumn{5}{|c|}{ Bus 1} \\
\hline & $3 X U D D S(\mathrm{mg} / \mathrm{mi})$ & SS 45Mph (mg/mi) & Idle $(\mathrm{mg} / \mathrm{min})$ & Bckgnd (mg/mile) & Bckgnd (mg/min) \\
\hline formaldehyde & 0.157 & 0.058 & 0.035 & 0.142 & 0.058 \\
\hline acetaldehyde & 0.083 & 0.038 & 0.028 & 0.139 & 0.056 \\
\hline acrolein & 0.000 & 0.000 & 0.000 & 0.000 & 0.000 \\
\hline acetone & 0.259 & 0.104 & 0.068 & 0.397 & 0.169 \\
\hline propionaldehyde & 0.011 & 0.006 & 0.005 & 0.020 & 0.007 \\
\hline butyraldehyde & 0.029 & 0.012 & 0.008 & 0.057 & 0.023 \\
\hline m-tolualdehyde & 0.042 & 0.004 & 0.007 & 0.000 & 0.000 \\
\hline methyl ethyl ketone & 0.000 & 0.000 & 0.000 & 0.000 & 0.000 \\
\hline methacrolein & 0.000 & 0.000 & 0.000 & 0.000 & 0.000 \\
\hline benzaldehyde & 0.000 & 0.000 & 0.000 & 0.000 & 0.000 \\
\hline crotonaldehyde & 0.000 & 0.000 & 0.000 & 0.000 & 0.000 \\
\hline valeraldehyde & 0.000 & 0.000 & 0.000 & 0.000 & 0.000 \\
\hline \multirow[t]{3}{*}{ hexanal } & 0.000 & 0.000 & 0.000 & 0.000 & 0.000 \\
\hline & \multicolumn{5}{|c|}{ Bus 2} \\
\hline & 3XUDDS (mg/mi) & $\mathrm{SS} 45 \mathrm{Mph}(\mathrm{mg} / \mathrm{mi})$ & Idle (mg/min) & Bckgnd (mg/mile) & Bckgnd (mg/min) \\
\hline formaldehyde & 0.082 & 0.046 & 0.033 & 0.034 & 0.044 \\
\hline acetaldehyde & 0.088 & 0.034 & 0.017 & 0.025 & 0.028 \\
\hline acrolein & 0.000 & 0.001 & 0.000 & 0.000 & 0.000 \\
\hline acetone & 0.141 & 0.074 & 0.037 & 0.045 & 0.061 \\
\hline propionaldehyde & 0.013 & 0.009 & 0.004 & 0.007 & 0.006 \\
\hline butyraldehyde & 0.000 & 0.000 & 0.002 & 0.000 & 0.000 \\
\hline m-tolualdehyde & 0.000 & 0.000 & 0.000 & 0.004 & 0.003 \\
\hline methyl ethyl ketone & 0.013 & 0.006 & 0.004 & 0.005 & 0.006 \\
\hline methacrolein & 0.011 & 0.000 & 0.000 & 0.000 & 0.000 \\
\hline benzaldehyde & 0.013 & 0.000 & 0.002 & 0.008 & 0.009 \\
\hline crotonaldehyde & 0.000 & 0.000 & 0.000 & 0.000 & 0.000 \\
\hline valeraldehyde & 0.008 & 0.002 & 0.001 & 0.003 & 0.002 \\
\hline hexanal & 0.019 & 0.008 & 0.002 & 0.000 & 0.000 \\
\hline
\end{tabular}

181 | P a g e 


\section{APPENDIX V- PAH RESULTS}

BDL: Below detection limitdenotes analyte concentration below $0.1 \mu \mathrm{g} / \mathrm{sample}$

\begin{tabular}{|c|c|c|c|c|c|c|}
\hline Targeted Analyte & $\begin{array}{l}\text { Bus } 1 \text { UDDS } \\
\text { (mg/test) }\end{array}$ & $\begin{array}{c}\text { Bus } 1 \text { UDDS } \\
\text { Background } \\
\text { (mg/test) }\end{array}$ & $\begin{array}{l}\text { Bus } 160 \text { min } \\
\text { Idle (mg/test) }\end{array}$ & $\begin{array}{c}\text { Bus } 160 \text { min } \\
\text { Idle } \\
\text { Background } \\
\text { (mg/test) }\end{array}$ & $\begin{array}{c}\text { Bus } 1 \text { SS 45MPH } \\
\text { (mg/test) }\end{array}$ & $\begin{array}{c}\text { Bus } 1 \text { SS } 45 \\
\text { MPH } \\
\text { Background } \\
\text { (mg/test) }\end{array}$ \\
\hline Naphthalene & $1.814 \mathrm{E}-01$ & 4.261E-02 & 2.896E-01 & 6.319E-02 & 4.704E-01 & 3.209E-01 \\
\hline Acenaphthylene & BDL & $\mathrm{BDL}$ & $\mathrm{BDL}$ & $\mathrm{BDL}$ & $\mathrm{BDL}$ & $\mathrm{BDL}$ \\
\hline Acenaphthene & $\mathrm{BDL}$ & $\mathrm{BDL}$ & $\mathrm{BDL}$ & $\mathrm{BDL}$ & 2.464E-02 & $\mathrm{BDL}$ \\
\hline Fluorene & BDL & $\mathrm{BDL}$ & 1.991E-02 & BDL & $4.032 E-02$ & $\mathrm{BDL}$ \\
\hline Phenanthrene & $3.584 \mathrm{E}-02$ & $\mathrm{BDL}$ & 4.706E-02 & $1.986 \mathrm{E}-02$ & 1.277E-01 & $\mathrm{BDL}$ \\
\hline Anthracene & BDL & $\mathrm{BDL}$ & BDL & BDL & BDL & $\mathrm{BDL}$ \\
\hline Fluoranthene & BDL & $\mathrm{BDL}$ & BDL & BDL & $2.464 \mathrm{E}-02$ & BDL \\
\hline Pyrene & $\mathrm{BDL}$ & $\mathrm{BDL}$ & $\mathrm{BDL}$ & $\mathrm{BDL}$ & $\mathrm{BDL}$ & $\mathrm{BDL}$ \\
\hline Benzo[a]anthracene & $\mathrm{BDL}$ & $\mathrm{BDL}$ & $\mathrm{BDL}$ & $\mathrm{BDL}$ & $\mathrm{BDL}$ & $\mathrm{BDL}$ \\
\hline Chrysene & $\mathrm{BDL}$ & $\mathrm{BDL}$ & BDL & $\mathrm{BDL}$ & $\mathrm{BDL}$ & $\mathrm{BDL}$ \\
\hline Benzo[b]fluoranthene & $\mathrm{BDL}$ & $\mathrm{BDL}$ & $\mathrm{BDL}$ & $\mathrm{BDL}$ & $\mathrm{BDL}$ & $\mathrm{BDL}$ \\
\hline Benzo[k]fluoranthene & $\mathrm{BDL}$ & $\mathrm{BDL}$ & BDL & $\mathrm{BDL}$ & $\mathrm{BDL}$ & BDL \\
\hline Benzo[a]pyrene & $\mathrm{BDL}$ & $\mathrm{BDL}$ & $\mathrm{BDL}$ & $\mathrm{BDL}$ & $\mathrm{BDL}$ & $\mathrm{BDL}$ \\
\hline Indeno[1,2,3-cd]pyrene & $\mathrm{BDL}$ & $\mathrm{BDL}$ & $\mathrm{BDL}$ & $\mathrm{BDL}$ & $\mathrm{BDL}$ & $\mathrm{BDL}$ \\
\hline Dibenz $[a, h]$ anthracene & BDL & $\mathrm{BDL}$ & BDL & $\mathrm{BDL}$ & $\mathrm{BDL}$ & $\mathrm{BDL}$ \\
\hline Benzo[g,h,i]perylene & $\mathrm{BDL}$ & BDL & BDL & $\mathrm{BDL}$ & $\mathrm{BDL}$ & $\mathrm{BDL}$ \\
\hline 2-Methylnaphthalene & 8.960E-02 & $\mathrm{BDL}$ & $1.321 \mathrm{E}-01$ & $2.708 \mathrm{E}-02$ & $1.366 \mathrm{E}-01$ & $1.248 \mathrm{E}-01$ \\
\hline 1-Methylnaphthalene & 4.704E-02 & $\mathrm{BDL}$ & $6.878 \mathrm{E}-02$ & $\mathrm{BDL}$ & 7.391E-02 & 6.419E-02 \\
\hline 2,6-Dimethyl naphthalene & $3.584 \mathrm{E}-02$ & $\mathrm{BDL}$ & 4.344E-02 & $\mathrm{BDL}$ & 4.928E-02 & $1.961 \mathrm{E}-02$ \\
\hline 1-Methyl phenanthrene & BDL & $\mathrm{BDL}$ & BDL & $\mathrm{BDL}$ & BDL & $\mathrm{BDL}$ \\
\hline Benzo(e)pyrene & BDL & $\mathrm{BDL}$ & BDL & BDL & $\mathrm{BDL}$ & $\mathrm{BDL}$ \\
\hline Perylene & BDL & BDL & BDL & BDL & BDL & BDL \\
\hline
\end{tabular}

182 | P a g e 
APPENDIX V CONTD.

\begin{tabular}{|c|c|c|c|c|c|c|}
\hline Targeted Analyte & Bus 2 UDDS (mg/test) & $\begin{array}{c}\text { Bus } 2 \text { UDDS } \\
\text { Background } \\
\text { (mg/test) }\end{array}$ & $\begin{array}{l}\text { Bus } 260 \mathrm{~min} \\
\text { Idle }(\mathrm{mg} / \text { test })\end{array}$ & $\begin{array}{c}\text { Bus } 260 \mathrm{~min} \text { Idle } \\
\text { Background (mg/test) }\end{array}$ & $\begin{array}{c}\text { Bus } 2 \text { SS 45MPH } \\
(\mathrm{mg} / \text { test })\end{array}$ & $\begin{array}{c}\text { Bus } 2 \text { SS } 45 \\
\text { MPH } \\
\text { Background } \\
\text { (mg/test) }\end{array}$ \\
\hline Naphthalene & 1.0980E-01 & $5.7735 \mathrm{E}-02$ & 7.3961E-02 & $9.4244 \mathrm{E}-02$ & $3.5822 \mathrm{E}-01$ & $5.9878 \mathrm{E}-02$ \\
\hline Acenaphthylene & $\mathrm{BDL}$ & $\mathrm{BDL}$ & $\mathrm{BDL}$ & $\mathrm{BDL}$ & $\mathrm{BDL}$ & $\mathrm{BDL}$ \\
\hline Acenaphthene & $\mathrm{BDL}$ & $\mathrm{BDL}$ & $\mathrm{BDL}$ & $\mathrm{BDL}$ & $\mathrm{BDL}$ & $\mathrm{BDL}$ \\
\hline Fluorene & $\mathrm{BDL}$ & $\mathrm{BDL}$ & $\mathrm{BDL}$ & $\mathrm{BDL}$ & $\mathrm{BDL}$ & $\mathrm{BDL}$ \\
\hline Phenanthrene & 3.8093E-02 & $\mathrm{BDL}$ & $\mathrm{BDL}$ & $\mathrm{BDL}$ & $8.2838 \mathrm{E}-02$ & $\mathrm{BDL}$ \\
\hline Anthracene & $\mathrm{BDL}$ & $\mathrm{BDL}$ & $\mathrm{BDL}$ & $\mathrm{BDL}$ & $\mathrm{BDL}$ & $\mathrm{BDL}$ \\
\hline Fluoranthene & $\mathrm{BDL}$ & $\mathrm{BDL}$ & $\mathrm{BDL}$ & $\mathrm{BDL}$ & $\mathrm{BDL}$ & $\mathrm{BDL}$ \\
\hline Pyrene & $\mathrm{BDL}$ & $\mathrm{BDL}$ & $\mathrm{BDL}$ & $\mathrm{BDL}$ & $\mathrm{BDL}$ & $\mathrm{BDL}$ \\
\hline Benzo[a]anthracene & $\mathrm{BDL}$ & BDL & $\mathrm{BDL}$ & $\mathrm{BDL}$ & $\mathrm{BDL}$ & $\mathrm{BDL}$ \\
\hline Chrysene & $\mathrm{BDL}$ & $\mathrm{BDL}$ & $\mathrm{BDL}$ & $\mathrm{BDL}$ & $\mathrm{BDL}$ & $\mathrm{BDL}$ \\
\hline Benzo[b]fluoranthene & $\mathrm{BDL}$ & $\mathrm{BDL}$ & $\mathrm{BDL}$ & $\mathrm{BDL}$ & $\mathrm{BDL}$ & $\mathrm{BDL}$ \\
\hline Benzo[k]fluoranthene & $\mathrm{BDL}$ & $\mathrm{BDL}$ & $\mathrm{BDL}$ & $\mathrm{BDL}$ & $\mathrm{BDL}$ & $\mathrm{BDL}$ \\
\hline Benzo[a]pyrene & $\mathrm{BDL}$ & $\mathrm{BDL}$ & $\mathrm{BDL}$ & $\mathrm{BDL}$ & $\mathrm{BDL}$ & $\mathrm{BDL}$ \\
\hline Indeno[1,2,3-cd]pyrene & $\mathrm{BDL}$ & $\mathrm{BDL}$ & $\mathrm{BDL}$ & $\mathrm{BDL}$ & $\mathrm{BDL}$ & $\mathrm{BDL}$ \\
\hline Dibenz $[a, h] a n t h r a c e n e$ & $\mathrm{BDL}$ & $\mathrm{BDL}$ & $\mathrm{BDL}$ & $\mathrm{BDL}$ & $\mathrm{BDL}$ & $\mathrm{BDL}$ \\
\hline Benzo[g,h,i]perylene & $\mathrm{BDL}$ & $\mathrm{BDL}$ & $\mathrm{BDL}$ & $\mathrm{BDL}$ & $\mathrm{BDL}$ & $\mathrm{BDL}$ \\
\hline 2-Methylnaphthalene & $5.8260 \mathrm{E}-02$ & $2.2206 \mathrm{E}-02$ & $2.9136 \mathrm{E}-02$ & $\mathrm{BDL}$ & 7.1644E-02 & 2.6613E-02 \\
\hline 1-Methylnaphthalene & 2.9130E-02 & $\mathrm{BDL}$ & $\mathrm{BDL}$ & $\mathrm{BDL}$ & $3.8061 \mathrm{E}-02$ & $\mathrm{BDL}$ \\
\hline 2,6-Dimethyl naphthalene & $2.4648 \mathrm{E}-02$ & $\mathrm{BDL}$ & $\mathrm{BDL}$ & $\mathrm{BDL}$ & $2.2389 \mathrm{E}-02$ & $\mathrm{BDL}$ \\
\hline 1-Methyl phenanthrene & $\mathrm{BDL}$ & $\mathrm{BDL}$ & $\mathrm{BDL}$ & $\mathrm{BDL}$ & $\mathrm{BDL}$ & $\mathrm{BDL}$ \\
\hline Benzo(e)pyrene & $\mathrm{BDL}$ & $\mathrm{BDL}$ & $\mathrm{BDL}$ & $\mathrm{BDL}$ & $\mathrm{BDL}$ & $\mathrm{BDL}$ \\
\hline Perylene & $\mathrm{BDL}$ & $\mathrm{BDL}$ & $\mathrm{BDL}$ & $\mathrm{BDL}$ & $\mathrm{BDL}$ & $\mathrm{BDL}$ \\
\hline
\end{tabular}

183 | P a g e 


\section{APPENDIX V- DTT ANALYSIS RESULTS}

ND: Non-denuded PM sample

DTPA: DTPA results denote the presence of metal chelator to block the activity of metals towards DTT consumption.

D: Denuded PM sample

\begin{tabular}{|l|c|c|c|}
\hline \multicolumn{1}{|c|}{ Sample ID } & DTT Activity (nmole DTT/min/micg) & Lower 95\% & Upper 95\% \\
\hline Bus 1 UDDS ND Bckgnd & 0.000 & 0.000 & 0.000 \\
\hline Bus 1 UDDS ND & 0.011 & 0.008 & 0.013 \\
\hline Bus 1 UDDS ND Bckgnd + DTPA & 0.000 & 0.000 & 0.000 \\
\hline Bus 1 UDDS ND + DTPA & 0.000 & 0.000 & 0.000 \\
\hline Bus 2 UDDS ND Bckgnd & 0.000 & 0.000 & 0.000 \\
\hline Bus 2 UDDS ND & 0.008 & 0.005 & 0.011 \\
\hline Bus 2 UDDS ND Bckgnd + DTPA & 0.000 & 0.000 & 0.000 \\
\hline Bus 2 UDDS ND + DTPA & 0.001 & 0.000 & 0.002 \\
\hline Bus 1 \& 2 Idle ND Bckgnd & 0.000 & 0.000 & 0.000 \\
\hline Bus 1 \& 2 Idle ND & 0.007 & 0.006 & 0.009 \\
\hline Bus 1 \& 2 Idle ND Bckgnd + DTPA & 0.000 & 0.000 & 0.000 \\
\hline Bus 1 \& 2 Idle ND + DTPA & 0.002 & 0.001 & 0.002 \\
\hline Bus 1 \& 2 45 MPH ND Bckgnd & 0.024 & 0.017 & 0.030 \\
\hline Bus 1 \& 2 45 MPH ND & 0.007 & 0.006 & 0.009 \\
\hline Bus 1 \& 2 45 MPH ND Bckgnd + DTPA & 0.000 & 0.000 & 0.000 \\
\hline Bus 1 \& 2 45 MPH ND + DTPA & 0.000 & 0.000 & 0.000 \\
\hline Bus 1 \& 2 UDDS D1-4 Bckgnd & 0.089 & 0.066 & 0.113 \\
\hline Bus 1 \& 2 UDDS D1-4 & 0.010 & 0.008 & 0.013 \\
\hline Bus 1 \& 2 UDDS D1-4 Bckgnd + DTPA & 0.000 & 0.000 & 0.000 \\
\hline Bus 1 \& 2 UDDS D1-4 + DTPA & 0.002 & 0.002 & 0.003 \\
\hline Bus 1 \& 2 Idle D1-4 Bckgnd & 0.000 & 0.000 & 0.000 \\
\hline Bus 1 \& 2 Idle D1-4 & 0.010 & 0.010 & 0.011 \\
\hline Bus 1 \& 2 Idle D1-4 Bckgnd + DTPA & 0.000 & 0.000 & 0.000 \\
\hline Bus 1 \& 2 Idle D1-4 + DTPA & 0.002 & 0.002 & 0.003 \\
\hline
\end{tabular}

184 | P a g e 


\begin{tabular}{|l|l|l|l|} 
Bus 1 \& 2 45 MPH D1-4 Bckgnd & 0.060 & 0.050 & 0.071 \\
\hline Bus 1 \& 245 MPH D1-4 & 0.015 & 0.013 & 0.017 \\
\hline Bus 1 \& 245 MPH D1-4 Bckgnd + DTPA & 0.011 & 0.006 & 0.016 \\
\hline Bus 1 \& 2 45 MPH D1-4 + DTPA & 0.003 & 0.003 & 0.004 \\
\hline
\end{tabular}




\section{APPENDIX VI- DHBA ANALYSIS RESULTS}

ND: Non-denuded PM sample

D: Denuded PM sample

\begin{tabular}{|c|c|c|c|}
\hline & DHBA formation (nmoles/ $\mathbf{\mu g} / \mathbf{m i n}$ ) & lower 95\% & upper 95\% \\
\hline Bus 1 UDDS Bkg ND & 0.0000 & 0.0000 & 0.0000 \\
\hline Bus 1 UDDS Sample ND & 0.0035 & 0.0031 & 0.0038 \\
\hline Bus 2 UDDS ND bkg & 0.0000 & 0.0000 & 0.0000 \\
\hline Bus 2 UDDS ND & 0.0024 & 0.0020 & 0.0028 \\
\hline Bus 1 \& 2 Idle ND bkg & 0.0000 & 0.0000 & 0.0000 \\
\hline Bus 1 \& 2 Idle ND & 0.0008 & 0.0007 & 0.0010 \\
\hline Bus 1 \& 2 45MPH ND Bkg & 0.0099 & 0.0086 & 0.0113 \\
\hline Bus 1 \& 2 45MPH ND & 0.0005 & 0.0004 & 0.0006 \\
\hline Bus 1 \& 2 UDDS D Bkg & 0.0089 & 0.0078 & 0.0100 \\
\hline Bus 1 \& 2 UDDS D & 0.0014 & 0.0012 & 0.0016 \\
\hline Bus 1 \& 2 IDLE D Bkg & 0.0000 & 0.0000 & 0.0000 \\
\hline Bus 1 \& 2 IDLE D & 0.0000 & 0.0000 & 0.0000 \\
\hline Bus 1\&Bus 2 45 MPH D bkg & 0.0000 & 0.0000 & 0.0000 \\
\hline Bus 1\&Bus 2 45 MPH D & 0.0003 & 0.0002 & 0.0003 \\
\hline
\end{tabular}




\section{APPENDIX VI- ALVEOLAR MACROPHAGE ANALYSIS RESULTS}

Chelex treatment refers to the removal of water soluble elements from the sample.

\begin{tabular}{|c|c|c|c|c|c|c|c|c|c|c|c|c|}
\hline \multirow[t]{2}{*}{ Test samples } & \multirow{2}{*}{$\begin{array}{c}\text { Extract } \\
\text { Treatment }\end{array}$} & \multirow{2}{*}{$\begin{array}{c}\text { PM } \\
\text { Mass } \\
(\mathrm{mg})\end{array}$} & \multirow{2}{*}{$\begin{array}{c}\text { Filter } \\
\text { Fractio } \\
\mathbf{n}\end{array}$} & \multicolumn{2}{|c|}{ Control Corrected } & \multirow{2}{*}{$\begin{array}{l}\text { ROS } \\
\text { Volum } \\
\text { e (mL) }\end{array}$} & \multirow{2}{*}{$\begin{array}{c}\text { Extractio } \\
n \\
\text { Volume }\end{array}$} & \multirow{2}{*}{$\begin{array}{c}\text { Dilutio } \\
\mathbf{n} \\
\text { Factor }\end{array}$} & \multicolumn{2}{|c|}{$\begin{array}{c}\mu \mathrm{g} \text { Zymosan Units I } \\
\text { Composite }\end{array}$} & \multicolumn{2}{|c|}{$\begin{array}{c}\mu \mathrm{g} \text { Zymosan Units / mg } \\
\text { Composite }\end{array}$} \\
\hline & & & & $\begin{array}{c}\text { Value } \\
\text { (FU) }\end{array}$ & $\begin{array}{c}\text { Stde } \\
\text { v }\end{array}$ & & & & Change & Stdev & Change & Stdev \\
\hline Bus 1, 3X UDDS, non-denuded & None & 0.172 & 1.0 & 727 & 132 & 0.10 & 5.25 & 1.0 & 463.1 & 95.7 & 2692.2 & 556.6 \\
\hline Bus $1,3 \times$ UDDS, non-denuded & Chelexed & 0.172 & 1.0 & 609 & 128 & 0.10 & 5.25 & 1.0 & 382.1 & 92.0 & 2221.5 & 534.7 \\
\hline Bus $1,3 \times$ UDDS, denuded & None & 0.148 & 1.0 & 100 & 36 & 0.10 & 5.25 & 1.0 & 32.9 & 30.0 & 221.4 & 201.4 \\
\hline Bus $1,3 \times$ UDDS, denuded & Chelexed & 0.148 & 1.0 & 74 & 160 & 0.10 & 5.25 & 1.0 & 15.1 & 111.0 & 101.5 & 746.8 \\
\hline $\begin{array}{c}\text { Bus 1, } 60 \text { min IDLE, non- } \\
\text { denuded }\end{array}$ & None & 0.057 & 1.0 & 320 & 27 & 0.10 & 5.25 & 1.0 & 212.9 & 30.6 & 3708.4 & 533.6 \\
\hline $\begin{array}{c}\text { Bus 1, } 60 \text { min IDLE, non- } \\
\text { denuded }\end{array}$ & Chelexed & 0.057 & 1.0 & 298 & 160 & 0.10 & 5.25 & 1.0 & 195.4 & 128.9 & 3403.9 & 2245.4 \\
\hline Bus 1, 60 min IDLE, denuded & None & 0.038 & 1.0 & 269 & 104 & 0.10 & 5.25 & 1.0 & 172.4 & 85.3 & 4523.7 & 2237.5 \\
\hline Bus $1,60 \mathrm{~min}$ IDLE, denuded & Chelexed & 0.038 & 1.0 & 186 & 100 & 0.10 & 5.25 & 1.0 & 106.4 & 81.9 & 2793.4 & 2150.5 \\
\hline Bus 1, SS 45MPH, non-denuded & None & 0.074 & 1.0 & 25 & 20 & 0.10 & 3.50 & 1.0 & -10.8 & 12.7 & -145.7 & 171.0 \\
\hline Bus $2,3 \mathrm{X}$ UDDS, non-denuded & None & 0.350 & 1.0 & 430 & 73 & 0.10 & 3.50 & 1.0 & 151.2 & 31.3 & 431.6 & 89.4 \\
\hline Bus 2, 3X UDDS, denuded & None & 0.137 & 1.0 & 288 & 35 & 0.10 & 3.50 & 1.0 & 94.4 & 17.5 & 685.5 & 126.9 \\
\hline Bus 2, SS 45MPH, non-denuded & None & 0.075 & 1.0 & 175 & 75 & 0.10 & 3.50 & 1.0 & 45.7 & 29.3 & 607.1 & 389.9 \\
\hline Bus 2, SS 45MPH, denuded & None & 0.005 & 1.0 & 10 & 179 & 0.10 & 3.50 & 1.0 & -15.6 & 67.1 & -3117.7 & 13412.2 \\
\hline $\begin{array}{c}\text { Bus 2, } 60 \text { min IDLE, non- } \\
\text { denuded }\end{array}$ & None & 0.292 & 1.0 & 1155 & 80 & 0.10 & 3.50 & 1.0 & 409.4 & 33.4 & 1398.2 & 114.2 \\
\hline Bus 2, $60 \mathrm{~min}$ IDLE, denuded & None & 0.241 & 1.0 & 1868 & 278 & 0.10 & 3.50 & 1.0 & 674.0 & 105.6 & 2789.8 & 437.0 \\
\hline
\end{tabular}

187 | P a g e 